\title{
Surface-Water-Quality Assessment of the Lower Kansas River Basin, Kansas And Nebraska: Dissolved Oxygen and Escherichia Coli Bacteria in Streams During Low Flow, July 1988 Through July 1989
}

\author{
By L.M. POPE
}

U.S. GEOLOGICAL SURVEY

Water-Resources Investigations Report 94-4077

Prepared as part of the

NATIONAL WATER-QUALITY

ASSESSMENT PROGRAM 


\section{U.S. DEPARTMENT OF THE INTERIOR}

\section{BRUCE BABBITT, Secretary}

U.S. GEOLOGICAL SURVEY

GORDON P. EATON, Director

For additional information write to:

Copies of this report can be purchased from:

U.S. Geological Survey Earth Science Information Center

District Chief U.S. Geological Survey Open-File Reports Section Box 25286, MS 517 4821 Quail Crest Place Denver Federal Center Lawrence, Kansas 66049-3839 Denver, Colorado 80225 


\section{CONTENTS}

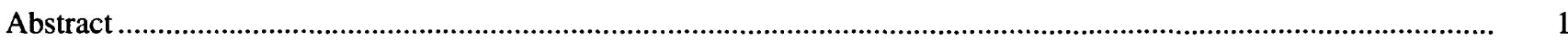

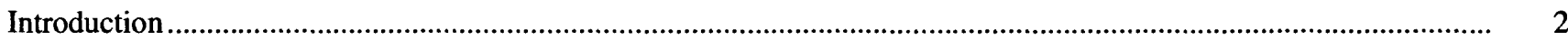

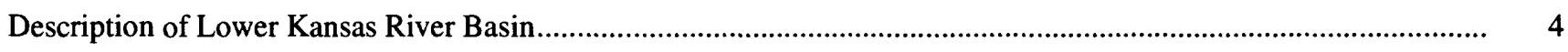

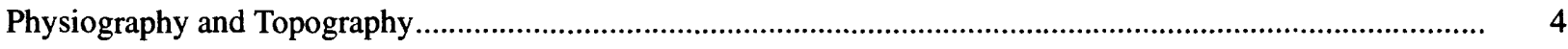

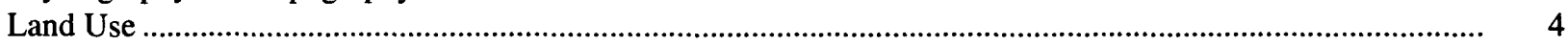

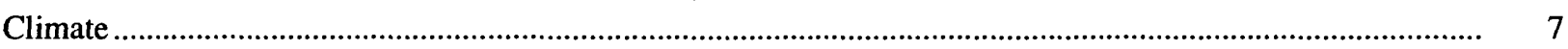

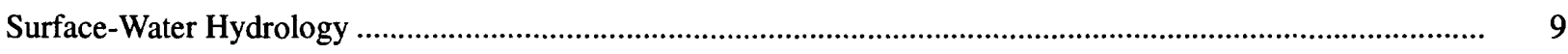

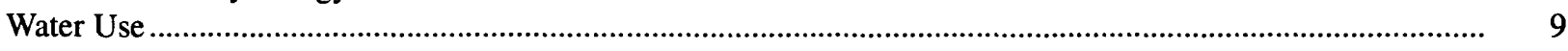

Stream Classification and Associated Water-Quality Criteria ............................................................ 11

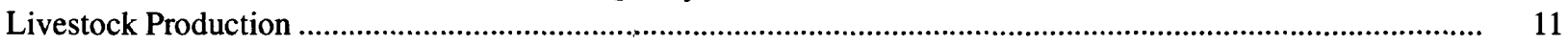

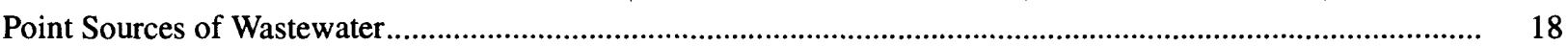

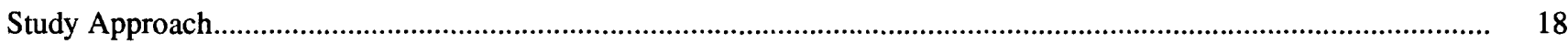

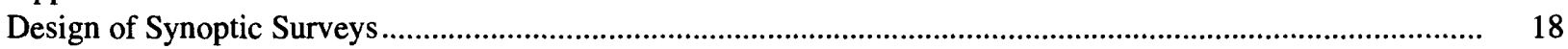

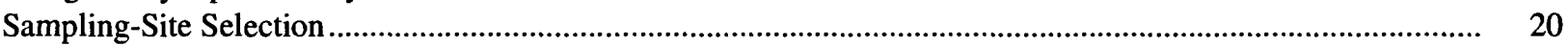

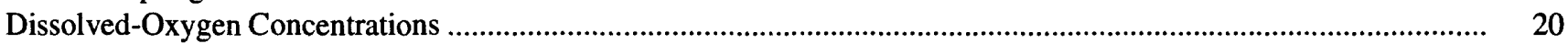

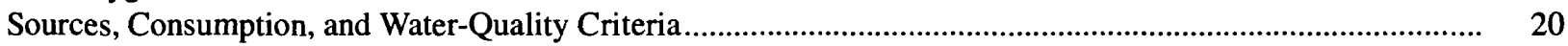

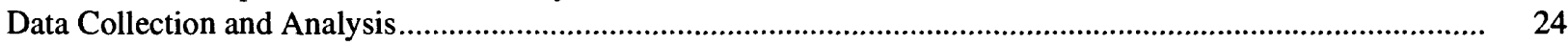

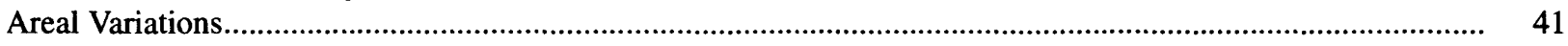

Diel Variability ................................................................................................................................

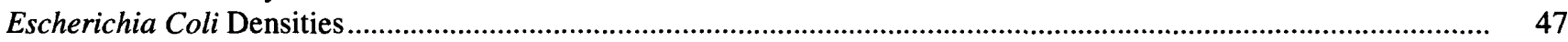

Sources and Water-Quality Criteria ............................................................................................................... 47

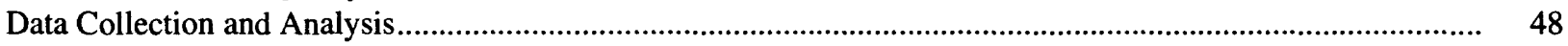

Associated Rainfall and Streamflow Characteristics ....................................................................................... 48

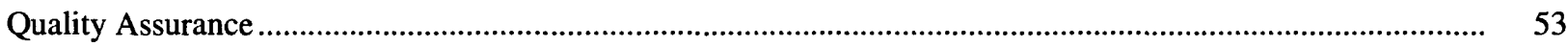

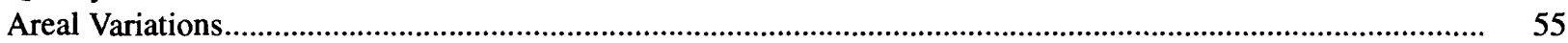

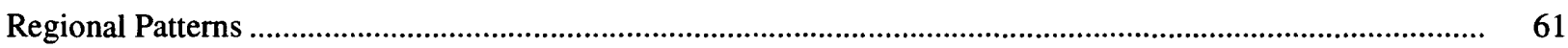

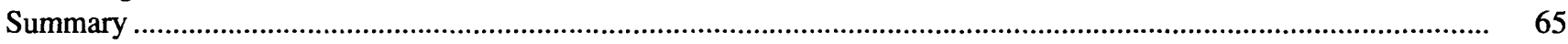

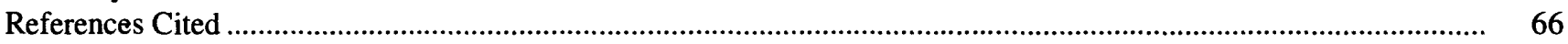

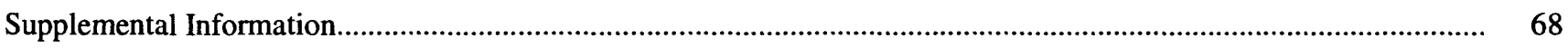

\section{FIGURES}

1. Map showing major streams, surface-water impoundments, cities, and other geographic features in and near lower Kansas River Basin, Kansas and Nebraska .......................................................................... 3

2. Map showing physiographic sections in lower Kansas River Basin................................................................. 5

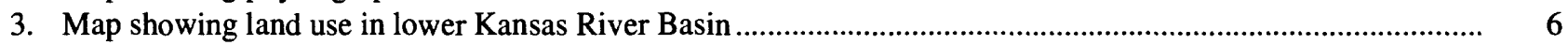

4. Graph showing total irrigated acreage in Adams, Butler, and Hamilton Counties, Nebr., 1930-85 ................... 7

5. Map and pie charts showing comparison of various land-use percentages in the Big Blue River, Little Blue

River, Kansas River, and Delaware River subbasins

6-11. Maps showing:

6. Location of wells permitted for irrigation withdrawal .........................................................................

7. Estimated percentage of cattle in feedlots, by county, in lower Kansas River Basin, January 1, $1988 \ldots \ldots \ldots . \quad 15$

8. Estimated cattle and hog densities, by county, in lower Kansas River Basin, January 1 and December 1, 1988

9. Location of municipal and industrial wastewater-treatment facilities with permitted discharges equal to or greater than 0.1 million gallons per day, $1990 \ldots$

10. Location of synoptic-sampling sites.

11. Areal distribution of predawn dissolved-oxygen concentrations, July 24-29, 1988, and location of municipal wastewater-treatment plants with permitted discharges of 1.0 million gallons per day or more. 


\section{FIGURES-Continued}

12. Graphs showing diel variability of dissolved-oxygen concentrations at selected synoptic-sampling sites in the lower Kansas River Basin for survey of July 1988

13. Graphs showing seasonal effect on the diel variability of dissolved-oxygen concentrations in the Big Blue River at Beatrice, Nebr., and the Kansas River at Lecompton, Kans.

14. Map showing areal distribution of Escherichia coli densities for synoptic survey of July 24-29, 1988, and location of municipal wastewater-treatment plants with permitted discharges of 1.0 million gallons per day or more

15-18. Graphs showing:

15. Distribution of Escherichia coli densities in water from the Big Blue River and its major tributaries for synoptic survey of July 24-29, 1988

16. Distribution of Escherichia coli densities in water from the Little Blue River and its major tributaries for synoptic survey of July 24-29, 1988

17. Distribution of Escherichia coli densities in water from the Kansas River and its major tributaries for synoptic survey of July 24-29, 1988

18. Comparison of the 25th, 50th (median), and 75th percentiles of Escherichia coli densities in the Big Blue River, Little Blue River, and Kansas River subbasins for synoptic survey of July 24-29, 1988

\section{TABLES}

1. Reported cattle and hog inventory and estimated inventory for the lower Kansas River Basin by county, 1988

2. Description of synoptic-sampling sites in the lower Kansas River Basin

3. Results of water-quality determinations and dissolved-oxygen concentrations at synoptic-sampling sites, July 1988 through June 1989

4. Escherichia coli densities and streamflow for synoptic surveys of July 1988 and July 1989

5. Rainfall characteristics at selected locations for synoptic surveys of July 1988 and July 1989

6. Streamflow-duration data at selected locations and sampling times.

7. Variation of Escherichia coli counts in two-plate duplicate-volume filtrations from samples collected during synoptic survey of July 1988

8. Results of ancillary chemical analyses of water samples collected July 1988 through June 1989 in the lower Kansas River Basin 


\section{CONVERSION FACTORS AND ABBREVIATIONS}

\begin{tabular}{rcl}
\hline \multicolumn{1}{r}{ Multiply } & By & To obtain \\
inch (in.) & 25.4 & millimeter \\
foot (ft) & 0.3048 & meter \\
yard (yd) & 0.9144 & meter \\
mile (mi) & 1.609 & kilometer \\
square mile (mi $\left.{ }^{2}\right)$ & 2.590 & square kilometer \\
acre & 4,047 & square meter \\
acre-foot (acre-ft) & 1,233 & cubic meter \\
acre-foot per year (acre-ft/yr) & 1,233 & cubic meter per year \\
cubic foot per second ( $\left(\mathrm{ft}^{3} / \mathrm{s}\right)$ & 0.02832 & cubic meter per second \\
gallon per minute (gal/min) & 0.06309 & liter per second \\
million gallons per day (Mgal/d) & 0.04381 & cubic meter per second \\
pound per square inch (lb/in $\left.{ }^{2}\right)$ & 6.895 & kilopascal \\
degree Fahrenheit $\left({ }^{\circ} \mathrm{F}\right)$ & $\left({ }^{1}\right)$ & degree Celsius $\left({ }^{\circ} \mathrm{C}\right)$ \\
\hline
\end{tabular}

${ }^{10} \mathrm{C}=5 / 9\left({ }^{\circ} \mathrm{F}-32\right)$.

${ }^{\circ} \mathrm{F}=9 / 5\left({ }^{\circ} \mathrm{C}\right)+32$. 
Vi Surface-Water-Quality Assessment of the Lower Kansas River Basin, Kansas and Nebraska: Dissolved Oxygen and Escherichia Coli Bacteria in Streams During Low Flow, July 1989 Through July 1989 


\title{
Surface-Water-Quality Assessment of the Lower Kansas River Basin, Kansas and Nebraska: Dissolved Oxygen and Escherichia Coli Bacteria in Streams During Low Flow, July 1988 Through July 1989
}

\author{
By L.M. Pope
}

\begin{abstract}
The 15,300-square-mile lower Kansas River Basin in Kansas and Nebraska was investigated, as one of the pilot study units of the U.S. Geological Survey's National Water-Quality Assessment (NAWQA) Program, to address a variety of water-quality issues. This report describes sanitary quality of streams as defined by concentrations of dissolved oxygen (DO) and densities of a fecal-indicator bacterium, Escherichia coli (E. coli).
\end{abstract}

Sixty-one surface-water sampling sites were chosen for this investigation. Synoptic surveys were conducted in July 1988, November 1988 , March 1989, and May 1989 to define the concentrations and diel and seasonal variability in concentrations of DO. Synoptic surveys were conducted in July 1988 and July 1989 to define densities of $E$. coli. Ancillary data included measurements of specific conductance, $\mathrm{pH}$, water temperature, barometric pressure, and concentrations of nutrients, total organic carbon, chlorophyll, and suspended sediment. Surveys were conducted during stable-flow, dry-weather conditions.

During the July 1988 synoptic survey for DO, emphasis was placed on the measurement of DO under maximum stress (high water temperature, low streamflow, and predawn conditions). Of 31 sites sampled just before dawn, 5 had DO concentrations less than the 5.0-milligrams-perliter, 1-day minimum warmwater criterion for early life stages as established by the U.S. Environmental Protection Agency (USEPA), and 4 of these 5 sites had concentrations less than the 3.0-milligrams-per-liter criterion for all other life stages. For all four synoptic surveys, a total of 392 DO determinations were made, and 9 (2.3 percent) were less than water-quality criteria. Concentrations of DO less than water-quality criteria in the study unit are localized occurrences and do not reflect regional differences in DO. The most severe DO deficiencies are the result of discharges from wastewater-treatment plants into small tributary streams with inadequate assimilation capacity. Algal respiratory demand in combination with reduced physical reaeration associated with extreme low flow probably also contributes to temporary, localized deficiencies.

Densities of $E$. coli were determined at 57 surface-water sampling sites during the synoptic survey in July 1988. Results indicate large regional differences in $E$. coli densities within the study unit. Densities of $E$. coli in water at 19 sites in the Big Blue River subbasin, exclusive of the Little Blue River subbasin, ranged from 120 to $260,000 \mathrm{col} / 100 \mathrm{~mL}$ (colonies per 100 milliliters), with a median density of $2,400 \mathrm{col} / 100 \mathrm{~mL}$. Densities at the 11 sites in the Little Blue River ranged from 100 to $30,000 \mathrm{col} / 100 \mathrm{~mL}$, with a median density of $940 \mathrm{col} / 100 \mathrm{~mL}$. Densities at the 27 sites in the Kansas River subbasin ranged from less than 1 to $1,000 \mathrm{col} / 100 \mathrm{~mL}$, with a median density of $88 \mathrm{col} / 100 \mathrm{~mL}$. Densities at 
84 percent of the sites in the Big Blue River subbasin exceeded the USEPA $E$. coli criterion of $576 \mathrm{col} / 100 \mathrm{~mL}$ for infrequently used full-body contact recreation, and 53 percent exceeded the $2,000 \mathrm{col} / 100 \mathrm{~mL}$ fecal coliform criterion for uses other than full-body contact established by the Kansas Department of Health and Environment. Densities at 73 percent of the sites in the Little Blue River subbasin exceeded the $576 \mathrm{col} / 100 \mathrm{~mL}$ E. coli criterion, and 36 percent exceeded the $2,000 \mathrm{col} / 100 \mathrm{~mL}$ fecal coliform criterion. Densities at one of the sites in the Kansas River subbasin exceeded the $576 \mathrm{col} / 100 \mathrm{~mL}$ E. coli criterion, and none exceeded the $2,000 \mathrm{col} / 100 \mathrm{~mL}$ fecal-coliform criterion.

The largest densities of $E$. coli in the study unit were the result of discharges from municipal wastewater-treatment plants; however, densities in the Big Blue and Little Blue River subbasins were generally larger than those in the Kansas River subbasin. These larger densities in the Big Blue and Little Blue River subbasins may have been the result of irrigation return flow from fields where manure was used as a soil amendment or the result of livestock production, which may have increased $E$. coli densities in ground-water discharge to the streams.

\section{INTRODUCTION}

In 1986, Congress appropriated funds for the U.S. Geological Survey to test and refine concepts for a National Water-Quality Assessment (NAWQA) Program. The NAWQA Program is designed to provide information on a variety of water-quality issues that include chemical contamination, acidification, eutrophication, salinity, sedimentation, and sanitary quality. This information will provide water managers, policy makers, and the public with an improved scientific basis for evaluating the effectiveness of water-quality management programs and for projecting the likely effects of contemplated changes in land- and water-management practices.

The NAWQA Program is organized into study units on the basis of known hydrologic systems. The pilot phase of the assessment program included seven study units (see report cover) representing a diversity of hydrologic environments and water-quality conditions. The subject of this report is the lower Kansas River Basin in Kansas and Nebraska (one of the surface-water pilot studies), which includes the Kansas River and its drainage area downstream from the confluence of the Republican and Smoky Hill Rivers to the confluence with the Missouri River (fig. 1).

Dissolved-oxygen (DO) concentrations and sanitary quality, as defined by the occurrence of a fecal-indicator bacterium, are two surface-waterquality issues addressed by the NAWQA Program. Adequate concentrations of DO in surface water are essential for the survival and propagation of most forms of aquatic life. Factors that affect DO in surface water include stream characteristics, such as turbulence of flow, depth, slope, and water temperature; barometric pressure; extent and composition of the algal community and associated rates of photosynthesis and respiration; presence of reduced forms of inorganic constituents (ammonia, nitrites, sulfites, and sulfides); and the introduction of anthropogenic oxygen-demanding organic-carbon compounds (agricultural activities, feedlot operations, and wastewater discharges).

An evaluation of the sanitary quality of water is necessary to assess its use as a public-water supply and for recreational activities, such as swimming, wading, boating, and fishing. Surface water can carry many pathogenic organisms of fecal origin that cause diseases, such as cholera, typhoid fever, dysentery, and other related gastrointestinal disorders. The examination of water for specific pathogens can require elaborate, time-consuming procedures not suitable for readily assessing quality. Traditionally, assessments have relied on a simplier, membrane-filter procedure for the detection of a group of bacteria (fecal coliform or fecal Streptococcus) or bacterium (Escherichia coli) common to the intestinal tracts of humans and warmblooded animals. The presence of the measured "fecal-indicator bacteria," denotes contamination by fecal material and the possible presence of pathogenic microorganisms. The indicator bacteria chosen for this study is Escherichia coli (E. coli).

This report (1) describes the areal distribution of dissolved-oxygen concentrations and $E$. coli bacteria in streams of the lower Kansas River Basin based on synoptic-sampling surveys conducted from July 1988 through July 1989 and (2) discusses the possible 


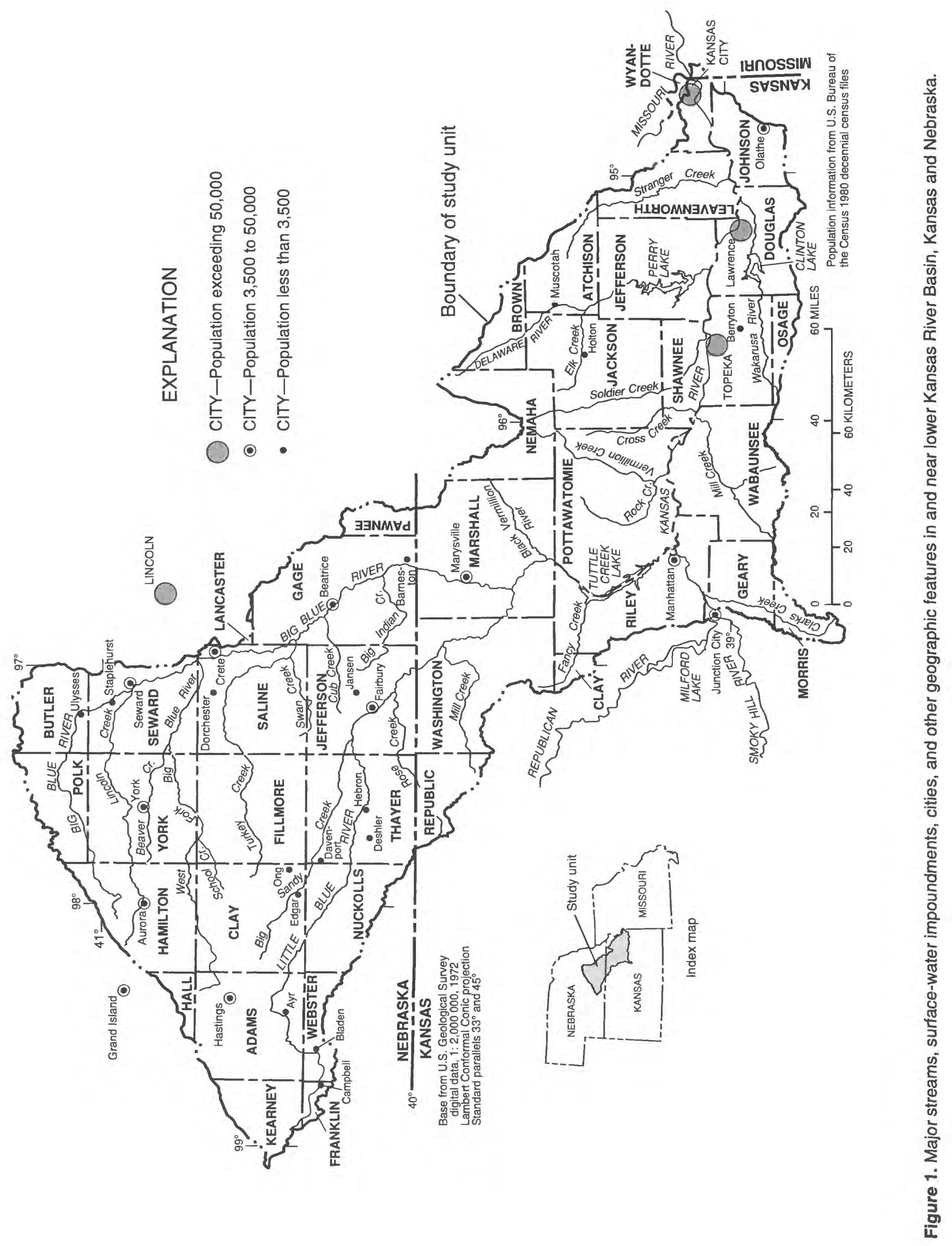


causal factors related to the observed water-quality conditions. Synoptic-sampling provides a view of water-quality conditions as they exist simultaneously over a broad geographical area. Synoptic sampling is conducted by sampling many sites within a short period of time to minimize variation in water-quality conditions from either natural (rainfall) or anthropogenic causes (point-source discharges or irrigation return flow).

\section{DESCRIPTION OF LOWER KANSAS RIVER BASIN}

The lower Kansas River Basin drains about $15,300 \mathrm{mi}^{2}$ and coincides with the area defined by the U.S. Water Resources Council as hydrologic subregion 1027 (Seaber and others, 1984). Although $7.5 \mathrm{mi}^{2}$ of the subregion lies within Missouri, drainage from this small area near the confluence of the Kansas and Missouri Rivers does not affect the quality of water used within the study unit and is not included in the study. The study unit does include the Big Blue and Little Blue River subbasins in Nebraska and Kansas, as well as subbasins of smaller tributaries to the 170-mi reach of the Kansas River from Junction City to Kansas City, Kans. (fig. 1).

The Kansas River is formed by the confluence of the Smoky Hill and Republican Rivers at Junction City, Kans. Three large reservoirs, Tuttle Creek Lake on the Big Blue River, Perry Lake on the Delaware River, and Clinton Lake on the Wakarusa River, lie within the Kansas part of the study unit (fig. 1). The following discussion of physiography and topography, land use, climate, surface-water hydrology, water use, and stream classification is summarized from Jordan and Stamer (1991).

\section{Physiography and Topography}

Land forms in the lower Kansas River Basin are characterized by the four physiographic sections shown in figure 2 (Fenneman, 1946). The High Plains and Plains Border Sections are part of the Great Plains Province. Smooth plains with little local relief dominate the High Plains Section; fluvial and eolian deposits that consist of sand, gravel, silt, and clay underlie this part of the study unit. The generally flat topography of the High Plains provides gentle stream gradients that contribute to only limited stream dissection and rather broad, poorly defined valleys. The flat topography also contributes to a lack of external drainage in some areas.

The Plains Border physiographic section is more dissected than the High Plains and thus has greater local relief. It is underlain by shale, sandstone, and limestone, and minor fluvial and eolian deposits. The drainage pattern in the Plains Border Section is more defined than in the High Plains Section. Stream channels are characteristically narrow, well established, and bounded by a perceptible series of terraces.

The Dissected Till Plains and Osage Plains Sections are part of the Central Lowland Province. The Dissected Till Plains Section is characterized by dissected deposits of glacial till that consist of silt, clay, sand, gravel, and boulders that overlie bedrock of primarily shale and limestone with some sandstone. Maximum local relief is from 300 to $500 \mathrm{ft}$ in the downstream part of the Big Blue River subbasin and generally less than $300 \mathrm{ft}$ elsewhere. Drainage channels are well entrenched by tributaries flowing south to the Kansas River. The Kansas River generally separates the Osage Plains from the Dissected Till Plains in a broad, flat alluvial valley bounded by rolling hills.

\section{Land Use}

Land use in the lower Kansas River Basin (fig. 3) is typical of the agricultural region of the midwestern United States. Types of agricultural uses are affected by the physiographic and topographic characteristics of the different parts of the study unit. Agriculture accounts for about 95 percent of the land use in the High Plains and Plains Border physiographic sections. More than 75 percent of the agricultural land in this part of the study unit is used for cultivated crops, and the balance is used principally as pasture. The principal crops in this part of the study unit, ordered by decreasing acreage, are corn, grain sorghum, wheat, and soybeans. The most intensely cultivated part of the study unit is the High Plains Section, in which about 85 percent of the agricultural land is cultivated. In this area, soil, topography, and ground-water availability are well suited for cultivated and irrigated crops. Although the area has long been used for cultivated crops, the amount of irrigation has increased severalfold since 1950, as shown in figure 4 for three representative counties. In the Plains Border Section of the study unit, about 70 percent of the agricultural land is 


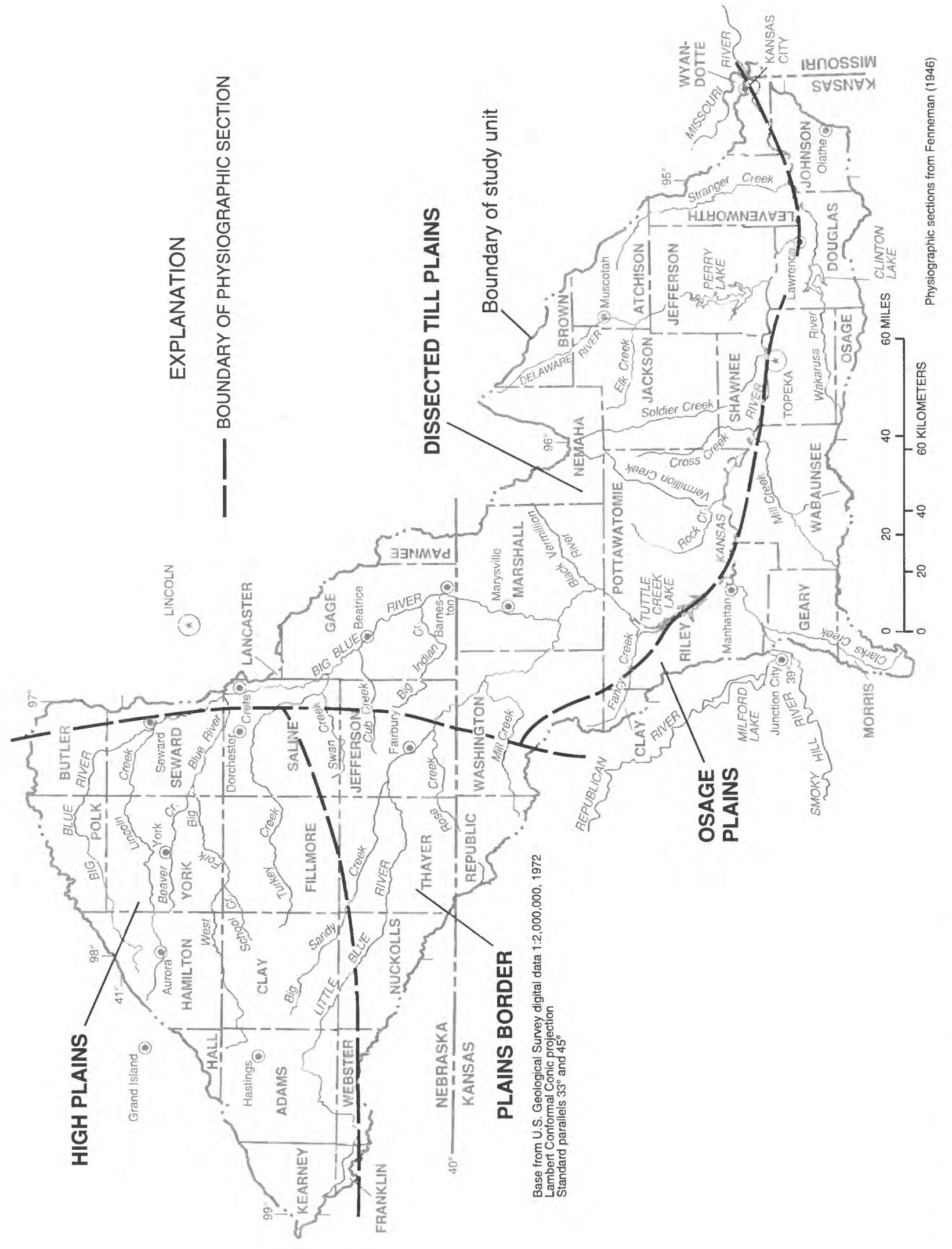

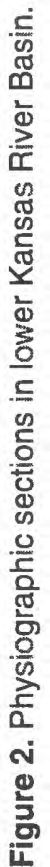




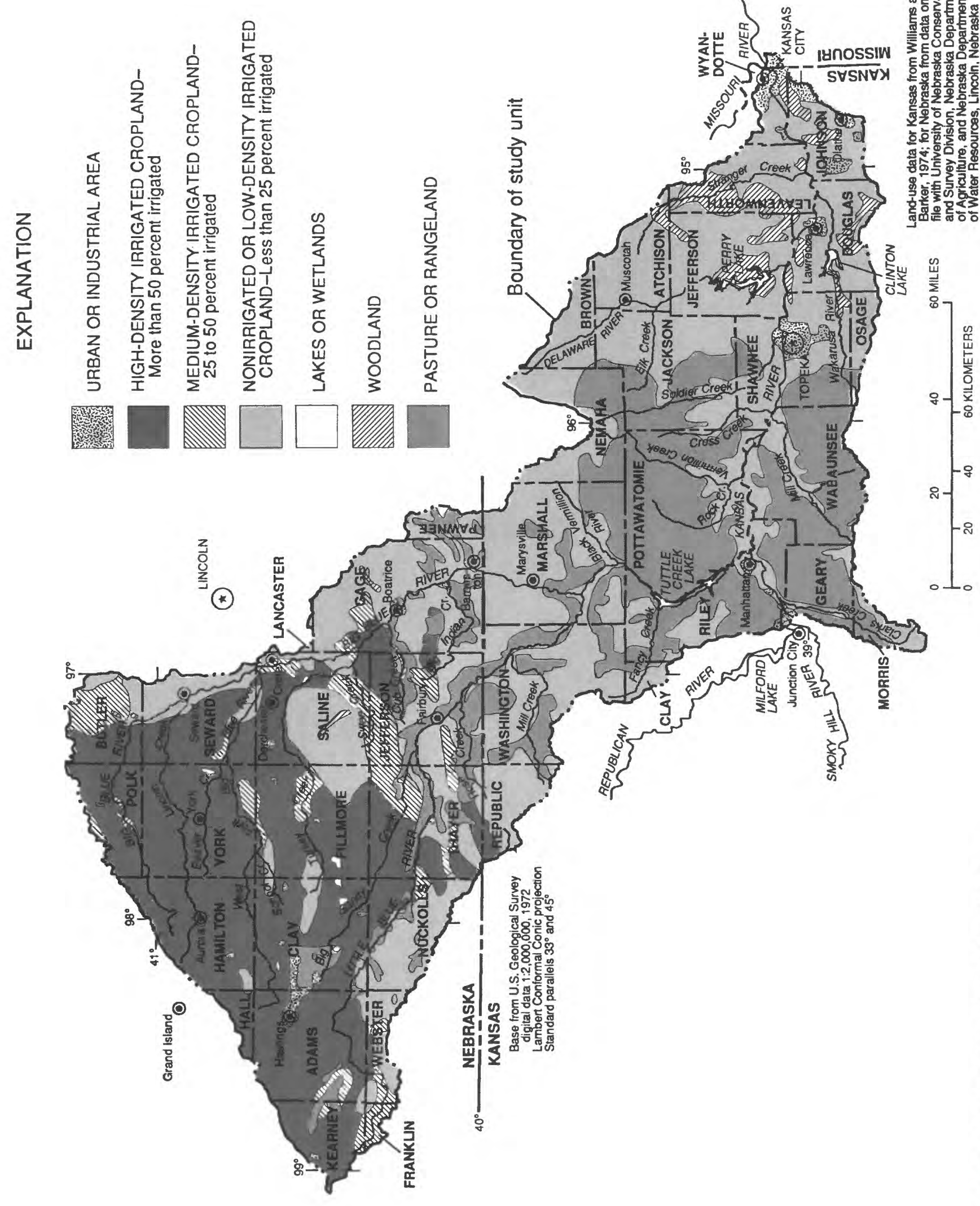

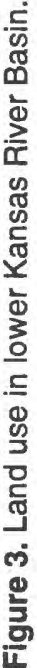




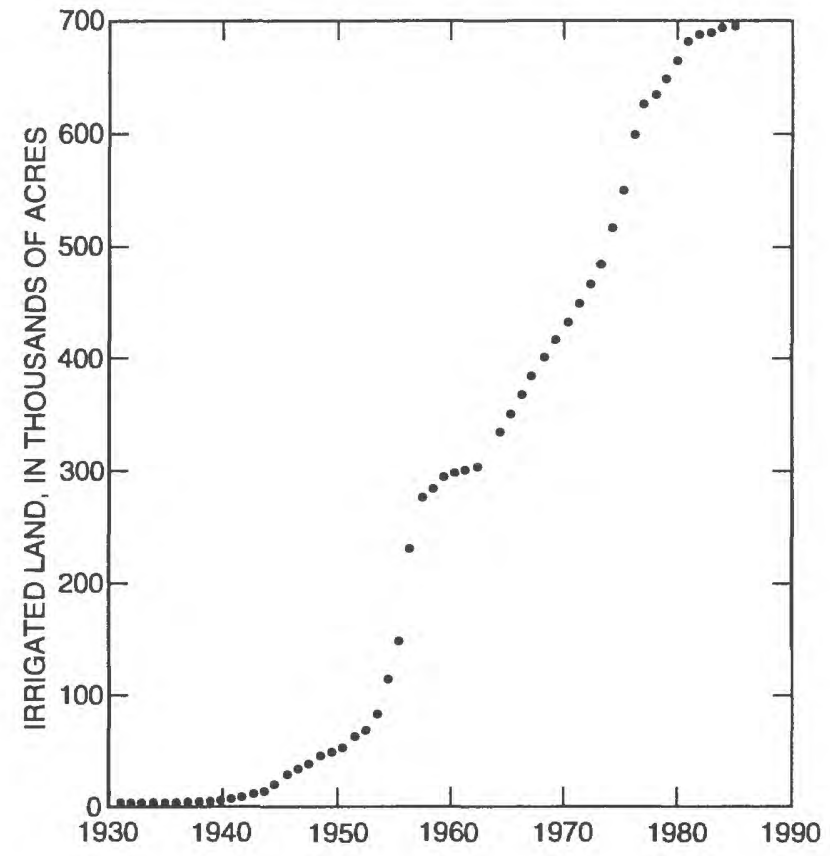

Figure 4. Total irrigated acreage in Adams, Butler, and Hamilton Counties, Nebr., 1930-85 (from data on file with the Nebraska Department of Water Resources, Lincoln).

used for nonirrigated, cultivated crops, and the remainder is used for pasture.

Land use in the Dissected Till Plains and the Osage Plains also is predominantly agricultural. These sections are characterized by more topographic relief and less ground-water availability than the area of the basin that lies in the High Plains and Plains Border Sections; thus, the area is less suited for cultivated and irrigated crops. The exception is the Kansas River flood plain and terrace area, which has low relief and substantial availability of ground water. Irrigation is practiced along much of the Kansas River in areas too small to be shown in figure 3. Principal crops in the Dissected Till Plains and Osage Plains are grain sorghum, wheat, corn, soybeans, and hay. The Flint Hills area in Riley, Geary, and Wabaunsee Counties, Kans., is mostly rangeland, and the remaining area in the Dissected Till Plains and Osage Plains is mixed cropland (30-60 percent) and pasture.
Agricultural land use in the principal subbasins of the study unit is shown in figure 5. Most of the irrigated area in the study unit occurs in the Big Blue River and Little Blue River subbasins. Little irrigated land (too little to show) occurs in the Kansas River subbasin. Pasture and rangeland are the predominant land uses in the Kansas River subbasin. The 51.8 percent of the subbasin in pasture and rangeland is more than twice the percentage of any of the other three subbasins.

Urban development represents a very small fraction of the total basin land use. The major urban and industrial areas in the basin are the Kansas part of the Kansas City metropolitan area, Topeka, and Lawrence, Kans. The industrial area near Hastings, Nebr. (fig. 3), is larger than the city itself, but development in the area is very low density. Although the Kansas City metropolitan area is at the downstream end of the basin and has little effect on the Kansas River, some of its water supplies are affected by activities in the basin. Other land uses, such as woodland and lakes or wetlands, also occupy a very small part of the total area of the basin. The population of the study unit was about 500,000 in 1980 (U.S. Bureau of the Census 1980 decennial census files).

\section{Climate}

Climate in the lower Kansas River Basin is characterized by hot, humid summers and cold winters with no particular dry season. July is normally the warmest month in the basin with a mean temperature of about $25^{\circ} \mathrm{C}$, and January is normally the coldest month with a mean temperature of about $-4^{\circ} \mathrm{C}$. Mean annual temperatures range from about $11^{\circ} \mathrm{C}$ in the northwestern part of the basin to about $12^{\circ} \mathrm{C}$ in the southeast. (Except as noted, climatic data in this report are from the National Oceanic and Atmospheric Administration, 1951-80.)

Precipitation in the basin is the most significant climatic factor affecting agriculture and surface-water availability because of both temporal and spatial variability. The 1951-80 mean annual precipitation ranged from about 24 in. in the northwestern part of the basin to about 36 in. in the southeast. Extreme variability, however, characterizes annual precipitation patterns. For example, during 1951 to 1980 , annual precipitation on large parts of the basin ranged from less than $15 \mathrm{in}$. to more than $50 \mathrm{in}$. The potential for drought, 


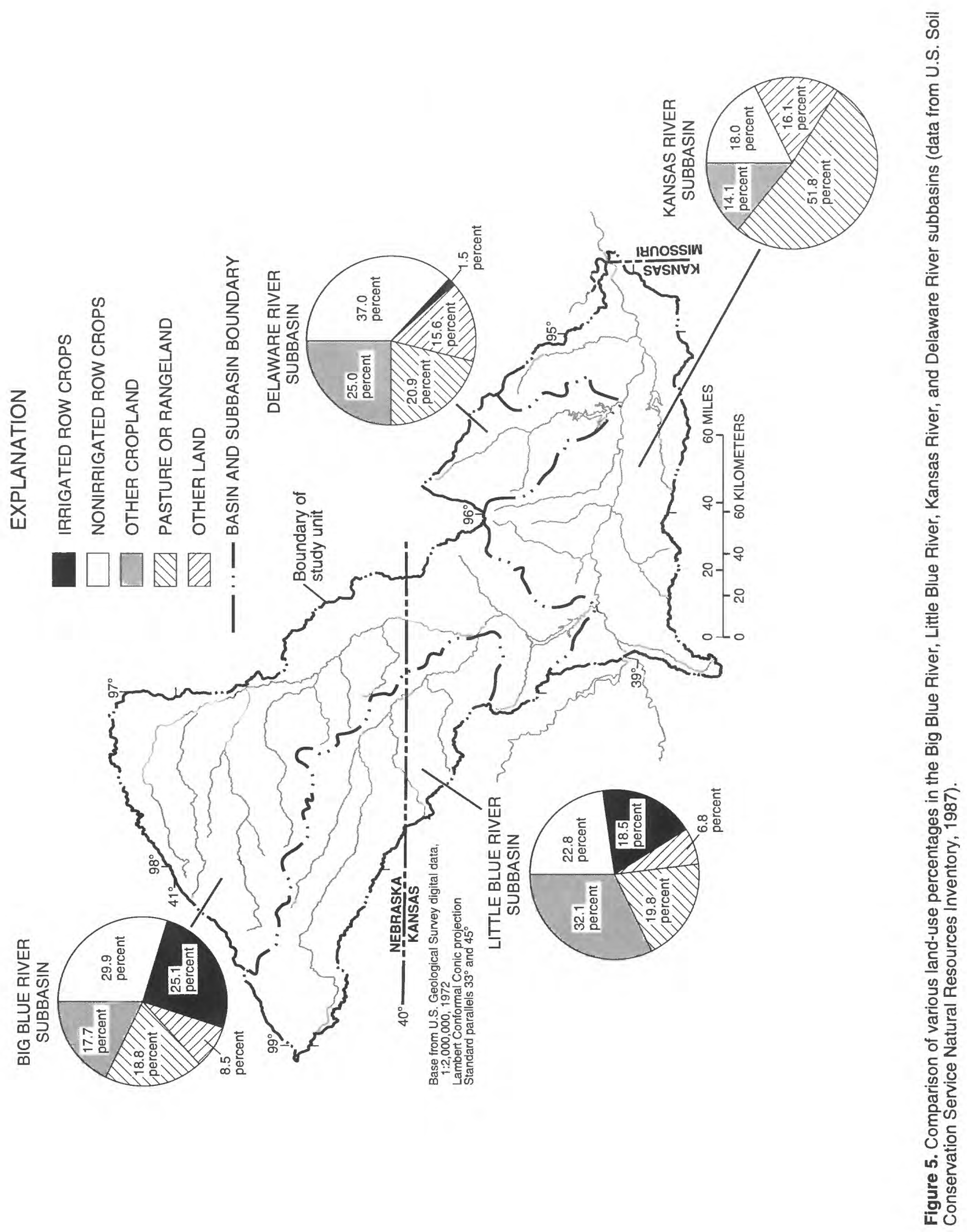


both short and long term, is always great within the basin. The potential for periodic flooding caused by excessive precipitation and runoff is equally great.

About 75 percent of the precipitation in the basin normally occurs during the warm season, April through September, which coincides for the most part with the growing season. Precipitation during the growing season, however, is not always sufficient to provide optimal soil-moisture conditions for most crops grown in the study unit. Thus, where water supplies are adequate, irrigation is a common practice.

\section{Surface-Water Hydrology}

The Republican and Smoky Hill Rivers, which join to form the Kansas River at Junction City, Kans., both begin in the plains of eastern Colorado and flow about 500 mi eastward to their confluence (fig. 1). Thus, the Kansas River at its beginning receives streamflow from a drainage area of about $45,000 \mathrm{mi}^{2}$. The Republican River, although it drains more than one-half of the area, provides about one-third of the mean flow (about $2,600 \mathrm{ft}^{3} / \mathrm{s}$ ) entering the lower Kansas River study unit, and the Smoky Hill River provides the other two-thirds of the flow.

The largest tributary downstream from Junction City is the Big Blue River, which originates in Nebraska ás does its principal tributary, the Little Blue River. The Big Blue River enters the Kansas River at Manhattan, Kans. Other principal tributaries that drain from the north to the Kansas River are Vermillion Creek, Soldier Creek, the Delaware River, and Stranger Creek. The drainage to the Kansas River from the south is much smaller than that from the north and includes Clarks and Mill Creeks and the Wakarusa River.

Runoff in the study unit varies areally as determined by precipitation, vegetation, topography, soil, and geology, and seasonally in response to precipitation and evapotranspiration. The 50-percent increase in mean annual precipitation from about $24 \mathrm{in}$. in the northwest to about 36 in. in the southeast is accompanied by a 350 -percent increase in mean annual runoff from less than 2 in. in the northwestern part of the study unit to almost 9 in. in the southeast. Mean monthly runoff is large in the spring and summer and smallest in the late fall and early winter.

The mean flow rate of the Kansas River at its confluence with the Missouri River during 1971-86 was about $8,600 \mathrm{ft}^{3} / \mathrm{s}$, of which the Big Blue River contributed about 27 percent; the Smoky Hill River, 19 percent; the Republican River, 12 percent; the Delaware River, 9 percent; and smaller tributaries, the remaining 33 percent.

Flow in the Big and Little Blue Rivers generally is well sustained during dry weather by ground-water contributions (Ellis, 1981, p. 44). Thus, surface-water quality during low flow is affected by ground-water quality although the effect is not quantitatively known. Although wells completed in sandstone, which underlies parts of the Plains Border, western Dissected Till Plains, and western Osage Plains physiographic sections, yield as much as $100 \mathrm{gal} / \mathrm{min}$ (Bayne, 1975), little is known about the quantity of ground water contributed to streams in these areas. Ground water is scarce in the uplands of the central and eastern parts of the Dissected Till Plains and Osage Plains where bedrock is primarily shale with thin layers of limestone and sandstone. Wells in buried-valley aquifers north of the Kansas River yield as much as $500 \mathrm{gal} / \mathrm{min}$ (Bayne, 1975). The extent of hydraulic connection of these aquifers to streams varies considerably within the area; thus, the effect of buriedvalley aquifers on the quantity and quality of water in the streams ranges from negligible to appreciable.

Considerable interchange of water occurs between the Kansas River and its 1- to 2.5-mi wide alluvial aquifer. During periods of high river stage, the river provides recharge to the aquifer. During lengthy dryweather periods, the alluvial aquifer contributes an estimated 1 to $4 \mathrm{ft}^{3} / \mathrm{s}$ of flow per river mile to the Kansas River (Fader, 1974). The exchange of water probably has a significant effect on quantity and quality of water in both the river and the aquifer; however, quantitative studies of those effects have not been conducted.

\section{Water Use}

Water use in the lower Kansas River Basin in 1985 totaled about 2.6 million acre-ft (calculated from data on file with the U.S. Geological Survey, Lawrence, Kans., and Lincoln, Nebr.). Irrigation withdrawals account for about 1.2 million acre-ft of the total water use and are predominantly ground water from the High Plains aquifer and the alluvial aquifer along the Kansas River and partly surface water from the Big and Little Blue Rivers and the Kansas River (fig. 6). 


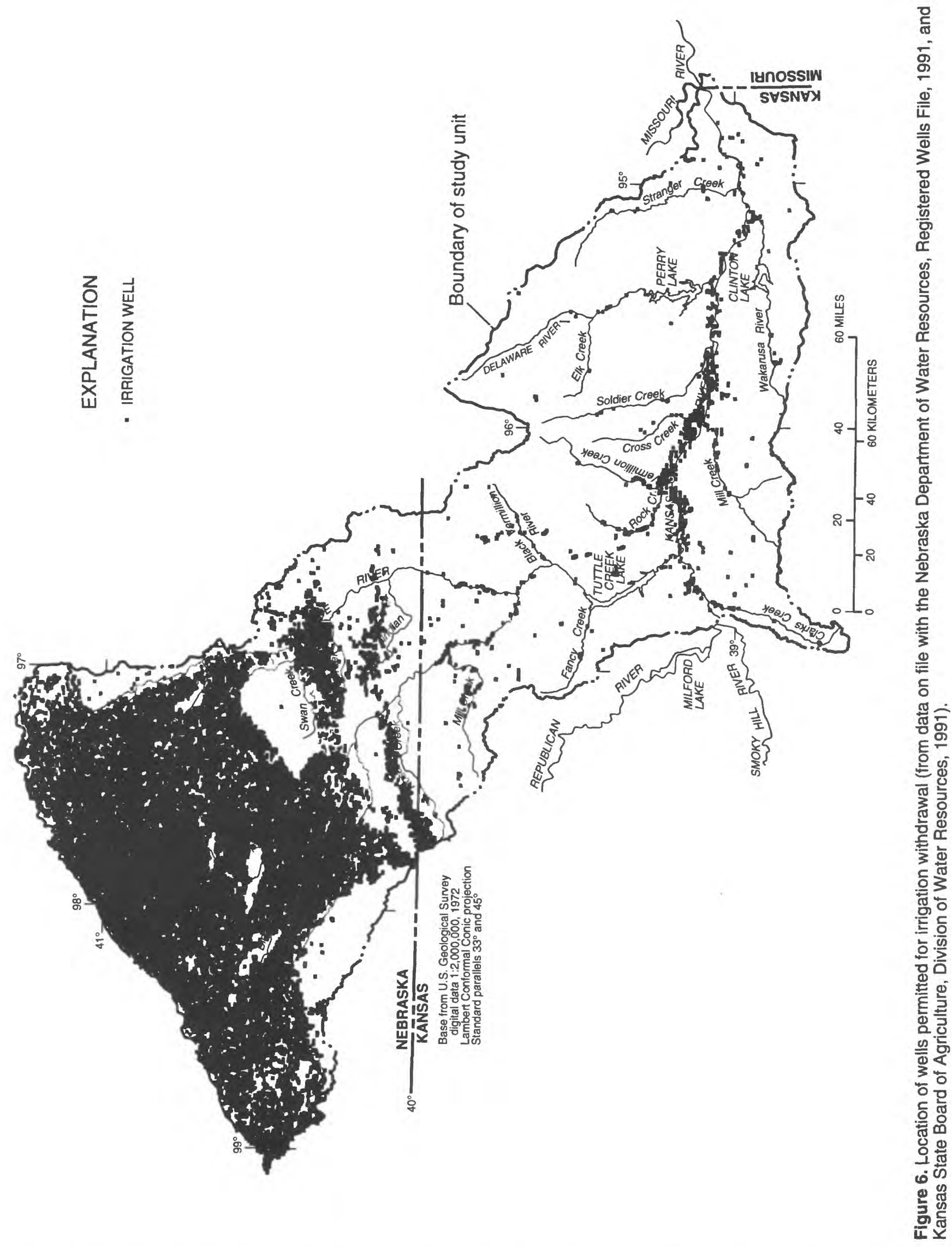

10 Surface-Water-Quality Assessment of the Lower Kansas River Basin, Kansas and Nebraska: Dissolved Oxygen and Escherichia Coll Bacteria in Streams During Low Flow, July 1988 Through July 1989 
Irrigation accounts for about 90 percent (about 0.8 million acre-ft) of the consumptive use of water in the basin. Other major uses having significant (more than 25 percent) consumptive components are selfsupplied industry and thermoelectric-power generation (115,000 acre-ft) and public supply (142,000 acre-ft).

Surface-water use in 1985 was about 1.3 million acre-ft/yr, which accounts for about 50 percent of the total water use. Surface water is used instream, nonconsumptively, for hydroelectric power ( 1.0 million acre-ft/yr) and offstream for self-supplied industry and thermoelectric-power generation $(91,000 \mathrm{acre}-\mathrm{ft} / \mathrm{yr})$, irrigation (82,000 acre-ft/yr), and public supplies $(86,000$ acre-ft/yr). Surface-water withdrawals for offstream use are mainly from the Kansas River, and the water is used within counties adjoining the river.

\section{Stream Classification and Associated Water-Quality Criteria}

Water-quality regulatory agencies of Kansas and Nebraska have taken slightly different approaches to stream classification. Although both States in 1986 had classified streams or stream segments for noncontact or secondary contact recreational use, Kansas also designated segments (defined as $200 \mathrm{yd}$ long) for contact recreation, whereas Nebraska applied the criteria for contact recreation to the same stream segment (miles long) as for other uses. Kansas designated streams for drinking-water use if their quality was potentially suitable for such use after appropriate treatment, whether or not the stream was being used currently for that purpose. Nebraska designated stream segments for public drinking water only if that use was being made currently. In the following abbreviated summary, the only uses discussed will be recreation, aquatic life, and drinking-water supply. Other uses designated by the State classifications, such as livestock watering, will not be discussed here.

Kansas stream classifications and associated water-quality criteria are described in detail by the Kansas Department of Health and Environment (Fromm and Wilk, 1988). The quality criteria, in general, are based on the harmful effects of substances that originate from artificial sources, turbidity, nutrients, $\mathrm{pH}$, temperature, dissolved oxygen, residual chlorine, toxic substances, and fecal coliform bacteria. The entire lengths of the Kansas and Big Blue Rivers are designated for noncontact recreation, aquatic life, and drinking-water supply. On other streams in the lower Kansas River Basin, 16 points are designated for contact recreation, and 37 streams are designated for noncontact recreation, aquatic life, and drinking-water supply.

Nebraska stream classifications in effect in 1986 and associated water-quality criteria are described by the Nebraska Department of Environmental Control (1986, p. 12, 29-33, and 55-59). The criteria considered chemical information, fecal indicator bacteria, toxic substances, suspended sediment, and biological information. Of the 415 stream mi in the lower Kansas River Basin within Nebraska that had been classified, $272 \mathrm{mi}$ were assigned the designation "Recreation Class B (secondary contact)," and none were assigned "Recreation Class A (primary contact)." In addition, 360 stream mi were assigned the aquatic-life designation "Warmwater Class A," 55 mi were assigned "Warmwater Class B," and none were assigned a "Coldwater" designation. No stream miles were designated for "Public Drinking Water Supply."

\section{Livestock Production}

The production of livestock is a major industry in Kansas and Nebraska. Cash receipts from livestock production and associated products in 1988 exceeded $\$ 4.2$ billion for Kansas and $\$ 5.3$ billion for Nebraska, ranking nationally sixth and second, respectively (Kansas State Board of Agriculture and U.S. Department of Agriculture, 1988-89). The total value of livestock production in the two states is approximately twice the value of crop production. The magnitude of this fact is striking in that, in 1988, Kansas ranked first, nationally, in the production of grain sorghum and wheat and eighth in corn, while Nebraska ranked second, third, and ninth in the production of corn, grain sorghum, and wheat (Nebraska Department of Agriculture and U.S. Department of Agriculture, 1989).

The vast majority of the livestock industry in Kansas and Nebraska centers around the production of cattle and hogs. As inventoried on January 1, 1988, the cattle and calf count was 5.9 million in Kansas (ranked second nationally) and 5.4 million in Nebraska (ranked third nationally). Hogs and pigs numbered 1.5 million for Kansas (10th nationally) and 4.15 million for Nebraska (fifth nationally). 
The extent and economic importance of the livestock industry in the lower Kansas River Basin mirror that delineated on a statewide basis. Estimates for the number of cattle and hogs, by county, in the study unit are provided in table 1. Estimates were calculated by multiplying the county inventory values provided in Kansas State Board of Agriculture and U.S.

Department of Agriculture (1988-89) and Nebraska Department of Agriculture and U.S. Department of Agriculture (1989) by the percentage of the county within the study unit. Overall, there were approximately 1.07 million cattle (as of January 1, 1988) and about 1.15 million hogs (as of December 1, 1988) in the study unit. The number of cattle in the Nebraska part of the basin $(563,200)$ was about 12 percent larger than the number in Kansas $(503,100)$. However, a much wider disparity existed between the two states in the number of hogs. The Kansas hog inventory $(323,000)$ was only 39 percent of the Nebraska inventory $(822,400)$. A large part of this disparity can be attributed to the exceptionally large inventory in Clay $(180,000)$, Fillmore $(98,000)$, Gage $(105,000)$, and York $(84,000)$ Counties in Nebraska.

Although total livestock inventory is an important characteristic for assessing surface-water quality, the extent of confined feeding operations (feedlots) and livestock density (head per square mile) provide more useful information in assessing probable or potential effect. Feedlot operations vary from simple open lots that process tens of head per year to more elaborate, totally enclosed, and vastly larger operations capable of processing hundreds to thousands of head per year. Most large operations are required by regulations to control runoff by the installation of earthen retention impoundments. Smaller operations may be exempt from the construction of retention structures if the operators show that the facility does not constitute a water-quality hazard.

The extent and intensity of feedlot operations is shown in figure 7 . The number of cattle on feed for the Nebraska counties for January 1, 1988 (table 1), although not directly provided in Nebraska Department of Agriculture and U.S. Department of Agriculture (1989), were estimated by multiplying the ratio of cattle on feed for the entire State on January 1, $1988(2,000,000)$, to the State total placed on feed for the whole year $(5,320,000)$ by the number placed on feed in each of the counties in the basin for the entire year (J.L. Aschwege, Nebraska Agricultural Statistics Service, oral commun., 1991). The computed state- wide ratio of 0.376 was assumed to be valid for those Nebraska counties within the study unit.

By far, the largest percentage of the cattle inventory contained in feedlots, by county, occurs in the upstream part of the basin, generally north of the Kansas-Nebraska border. The percentage of cattle on feed in Nebraska counties ranged from 8.9 to 79.2 percent (table 1 ), with a county average of 43.0 percent. Those counties with the most intense feeding operations are in the headwaters and middlestream reaches of the Big Blue, West Fork Big Blue, and Little Blue Rivers. The smallest percentages of cattle confined to feedlots are in the downstream part of the basin, south of the Kansas-Nebraska border. Percentage of cattle on feed in those counties in Kansas ranged from 0.5 to 27.9 percent, with a county average of 4.6 percent. This disparity between the upstream and downstream parts of the basin can be attributed mainly to physiographic and land-use characteristics. As shown in figure 2, much of the upstream part of the basin occurs in the High Plains physiographic section, which is generally flat with gentle stream gradients and limited stream dissection. Soils in the High Plains tend to be deep and fertile and sustain intensive crop production as indicated in figure 3. Rangeland is scarce. The corn, grain sorghum, and silage produced in this area provide an excellent, locally available source of livestock feed. Farther downstream, the Dissected Till Plains and Osage Plains dominate. Local relief increases considerably, soils are thinner, irrigation water becomes scarce, and pasture and rangeland become abundant. Given the changes in local physiography and topography and the desire to utilize available resources, the majority of cattle in the downstream part of the basin are grass fed and not confined routinely to feedlots.

Livestock density, head per square mile, varies considerably throughout the basin (fig. 8). Generally, densities increase from the southeast (downstream) part of the basin to the northwest (upstream); this generalization is most pronounced in the distribution of hog densities. The largest densities for both cattle and hogs occur in or near the headwaters of the Big Blue, West Fork Big Blue, and Little Blue Rivers where densities of both cattle and hogs exceed 100 and 120 head per square mile, respectively, in five Nebraska counties. 


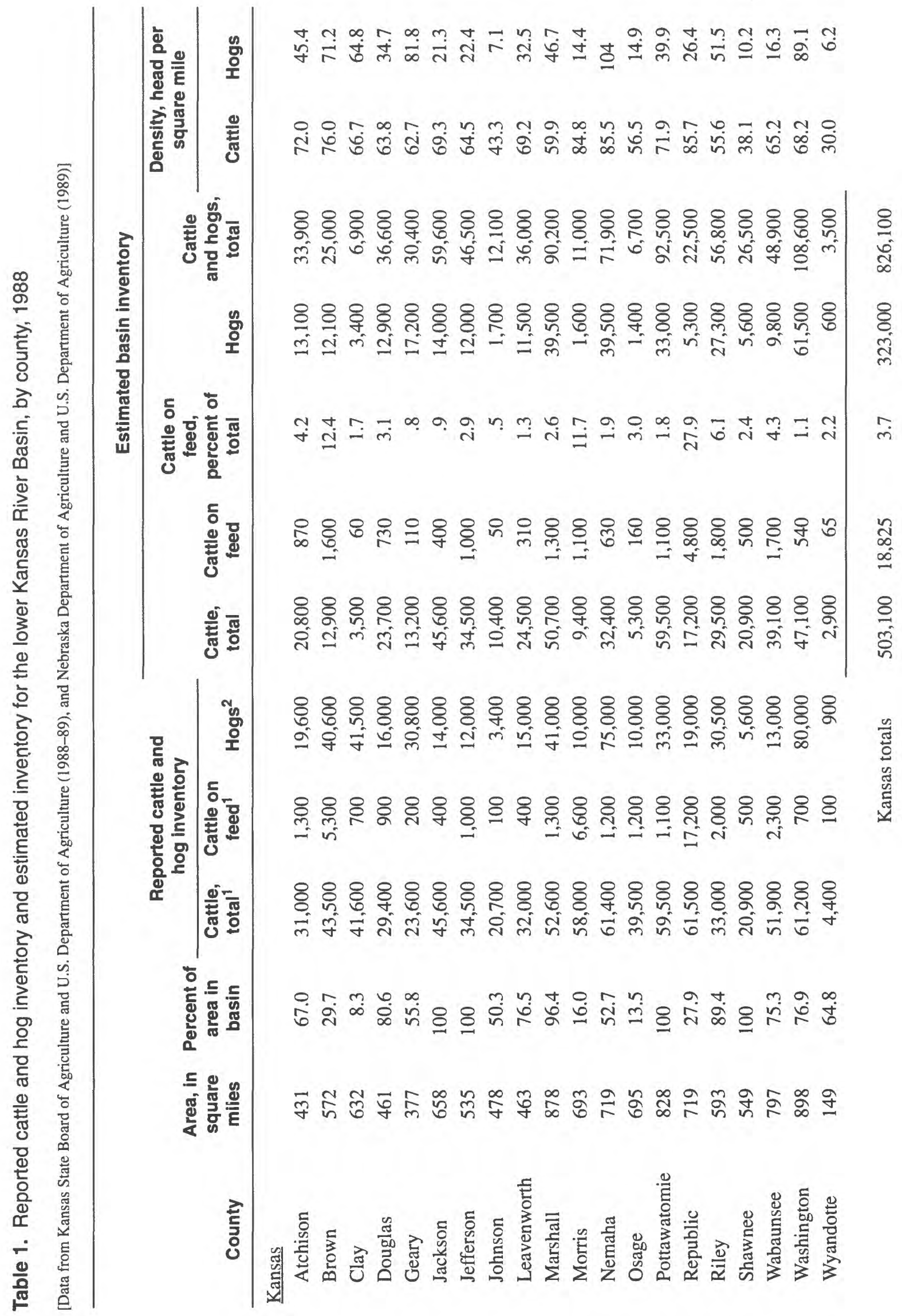




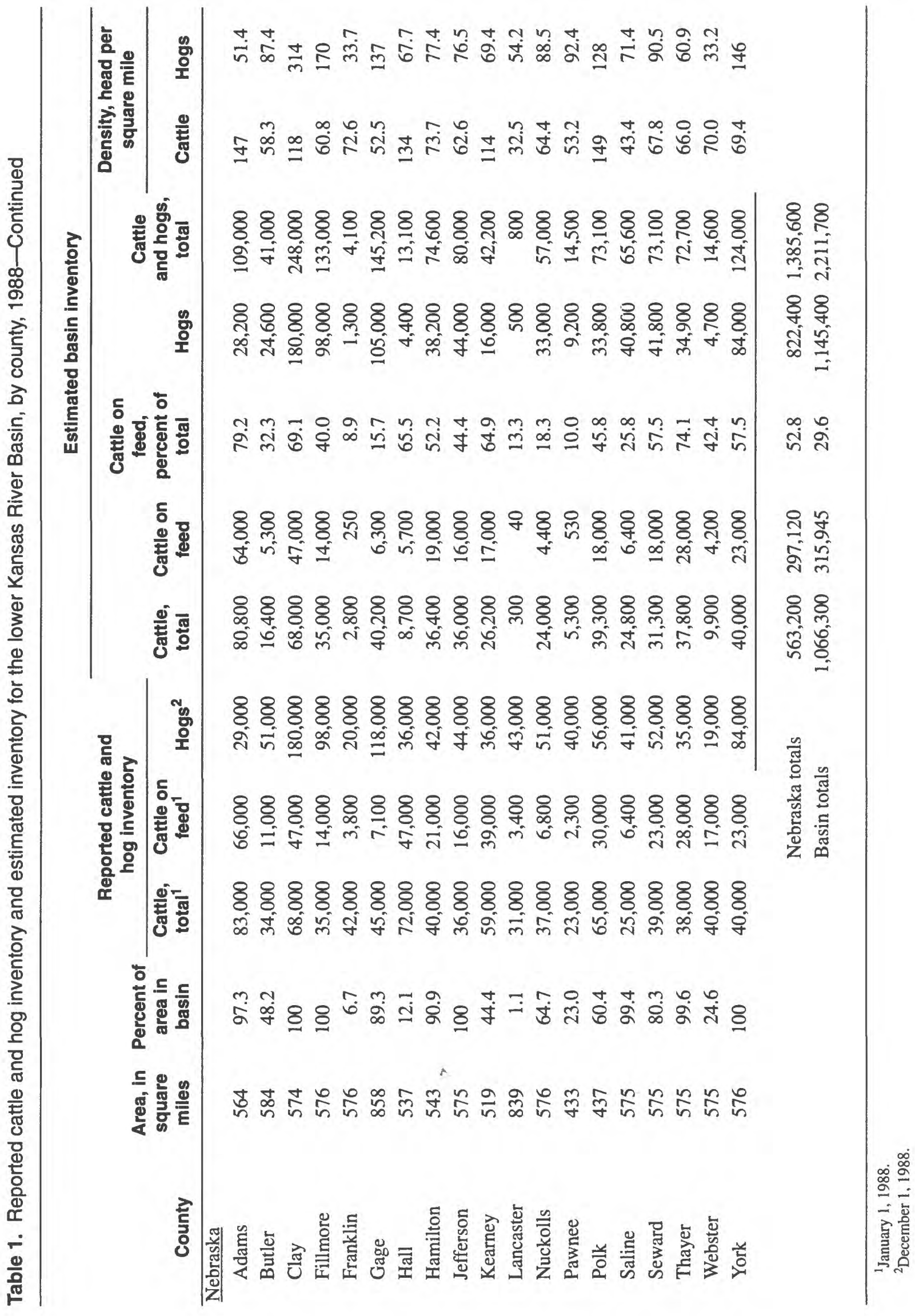




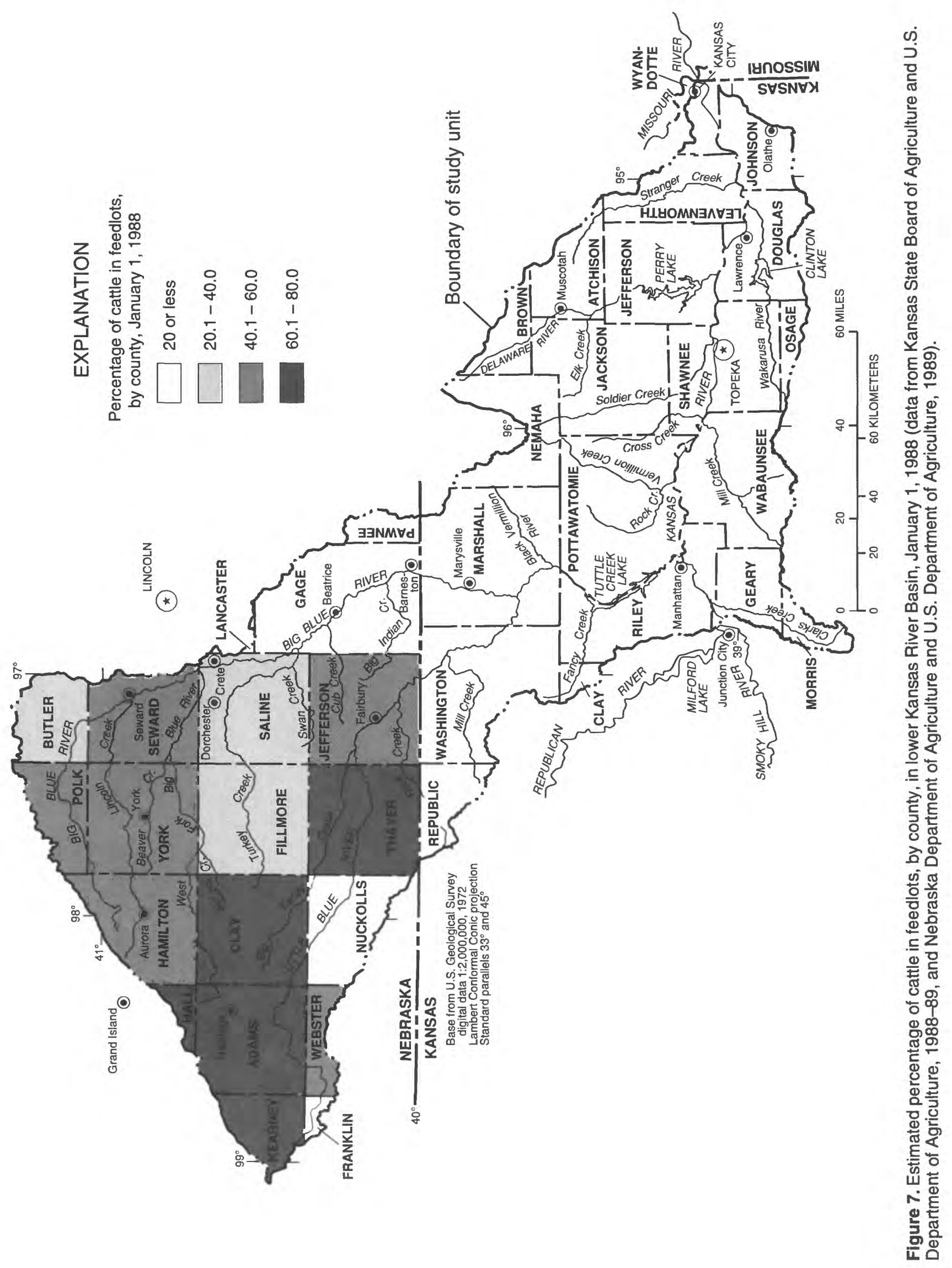



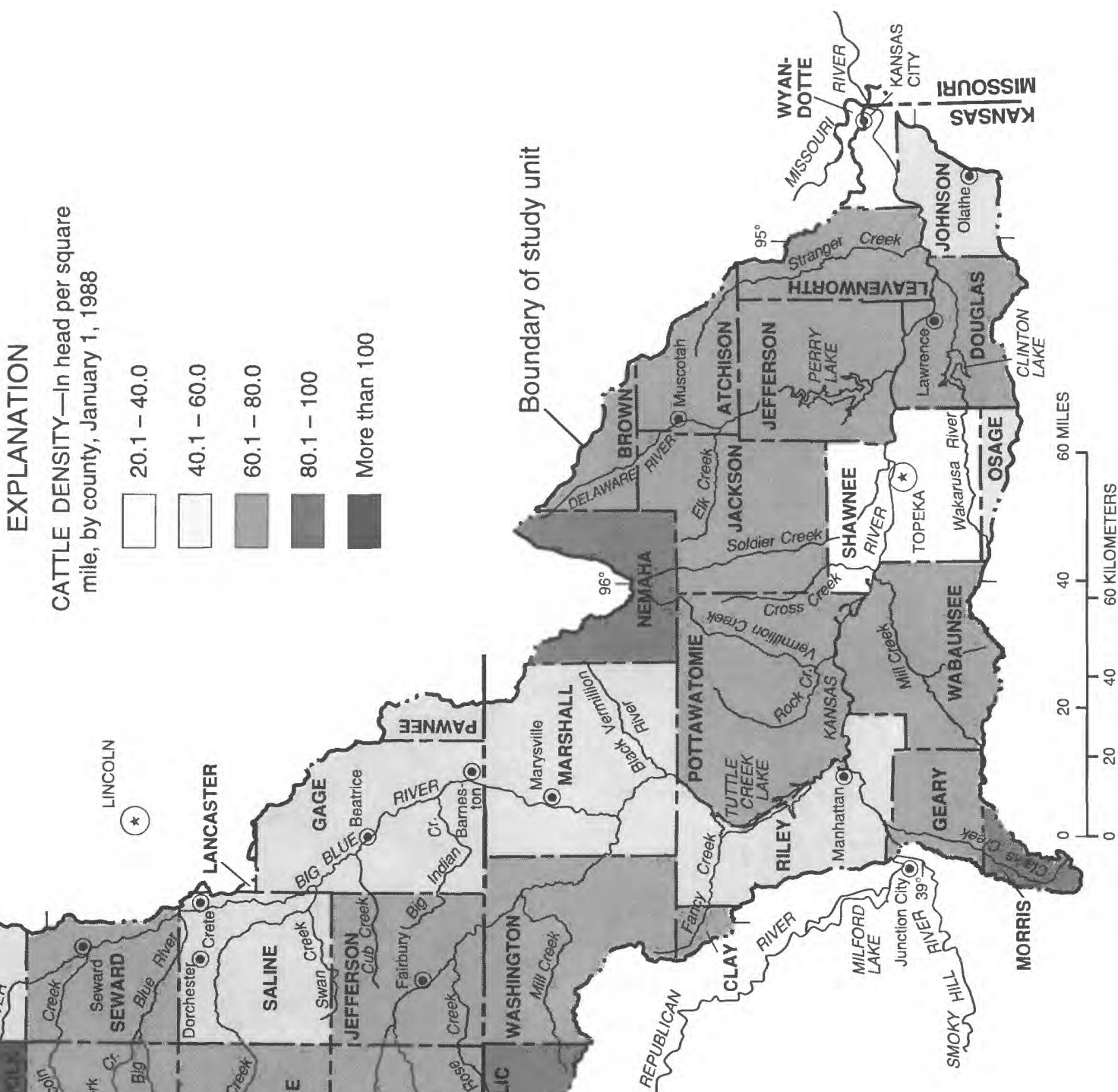

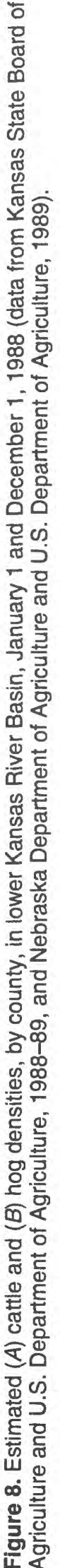

$\dot{\leftarrow}$

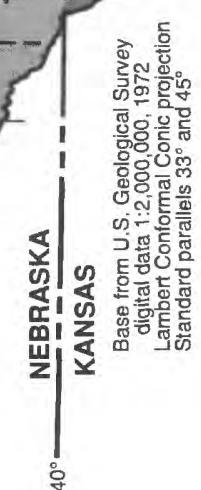

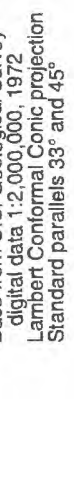




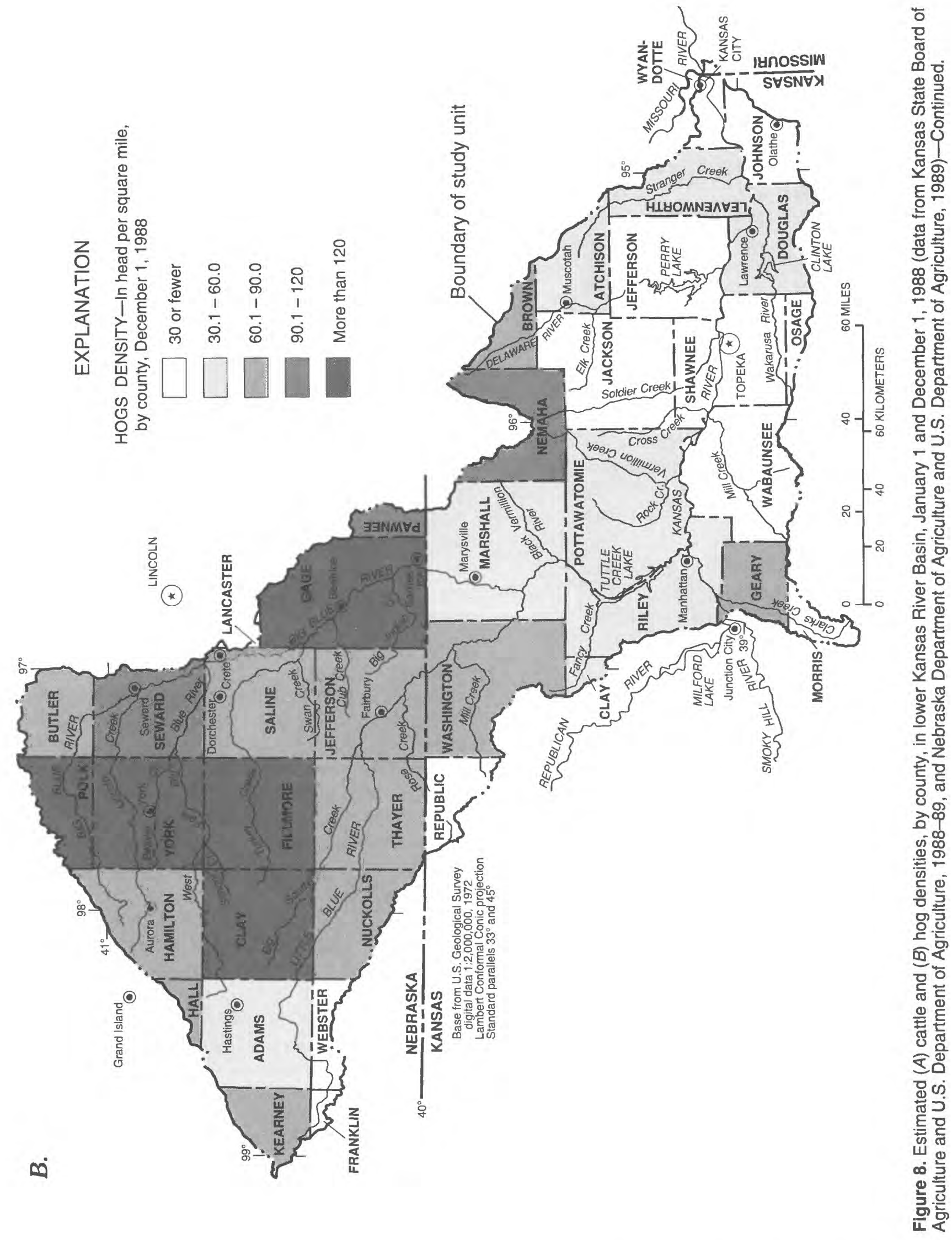




\section{Point Sources of Wastewater}

Wastewater that enters a stream at a discrete point is referred to as a point source and, as such, is not necessarily a function of geology, soils, or land use of a watershed basin. The U.S. Environmental Protection Agency (USEPA) issues discharge permits under the National Pollutant Discharge Elimination System (NPDES). These permits specify the allowed limits for rate of discharge and chemical composition of the wastewater effluent.

There are 28 municipal and industrial facilities in the study unit that are permitted by the USEPA to discharge wastewater at a rate of 1.0 or more $\mathrm{Mgal} / \mathrm{d}$. Of these facilities, 16 are municipal wastewatertreatment plants and 12 are industrial wastewatertreatment plants. The location of these 28 facilities, 42 municipal wastewater-treatment plants with permitted discharges of 0.1 to $0.9 \mathrm{Mgal} / \mathrm{d}$, and a municipal wastewater facility located outside the study unit on the Republican River is shown in figure 9. In addition to the facilities shown in figure 9, there are approximately 130 municipal wastewater-treatment facilities permitted to discharge less than $0.1 \mathrm{Mgal} / \mathrm{d}$. About 64 percent of these facilities are located in the Big Blue River system, with all but one facility of the remaining 36 percent located in the Kansas River system downstream of Manhattan, Kans.

Most of the larger (5.0 Mgal/d or greater) wastewater-treatment facilities are located along the main stem of the Kansas River and coincide with the main population centers in the study unit. All but one of these large facilities discharge directly into the Kansas River. One facility in Johnson County discharges into Turkey Creek, a tributary of the Kansas River that discharges into the Kansas River about $2.2 \mathrm{mi}$ upstream of its confluence with the Missouri River. One of the three largest (10 Mgal/d or more) municipal wastewater-treatment facilities, Kaw Point (28.0 Mgal/d) in Kansas City, Kansas, although physically located within the study unit, discharges into the Missouri River.

\section{STUDY APPROACH}

\section{Design of Synoptic Surveys}

The purpose of synoptic sampling is to provide information describing selected water-quality condi- tions throughout a large geographical area at many locations in as short a time as possible. In this study, each synoptic survey was completed within 6 days. Specifically, synoptic sampling provides (1) a means to increase the knowledge gained from an analysis of all available data; (2) a finer degree of resolution in describing water-quality conditions than provided by a small network of regularly sampled stations; (3) for an assessment of the spatial distribution of water-quality conditions in relation to such factors as physiography, topography, geology, land use, agricultural activities, and waste-management practices; and (4) a documentation of stream reaches where critical levels of DO and bacteria occur during hydrologic conditions most conducive to producing those levels (low flow and high water temperature).

This study was conducted to identify areas of the basin that may have DO deficiencies or degradation of sanitary quality (large $E$. coli densities) under conditions that would minimize the effects of runoff and maximize comparability of results throughout the basin. These requirements mandated that synoptic surveys be conducted under dry-weather, stable-flow conditions, or as nearly so as possible. Additionally, DO deficiencies are believed to be greatest during periods of maximum stress to the system-low streamflow (less dilution of waste), high water temperature (lower oxygen solubility), and maximum effect of algal respiration (usually just before sunrise). Also, nonpoint-source contamination during runoff can cause bacteria densities to fluctuate by several orders of magnitude; therefore, criteria for densities of $E$. coli have been established only for stable-flow, dryweather conditions. In the lower Kansas River Basin, these conditions are most common in mid- to latesummer.

A synoptic survey to define the distribution and magnitude of DO and $E$. coli was conducted in July 1988. Approximately 50 percent of the 61 sites were sampled just prior to sunrise (predawn) to assess DO concentrations under maximum stress conditions. Additionally, studies of diel variation of DO were conducted at 11 sites. DO data also were collected as a supplemental constituent during synoptic surveys of nutrient concentration in November 1988 and March and May-June 1989. During these surveys, two sites were sampled to define diel variation and seasonal changes. A followup survey for $E$. coli was conducted in July 1989 to verify results from the July 1988 survey. Ancillary data for selected DO and $E$. coli 
岁

으 뚬

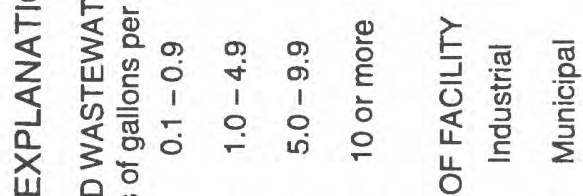

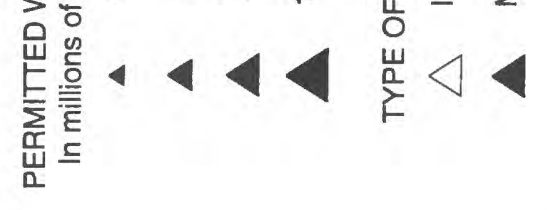

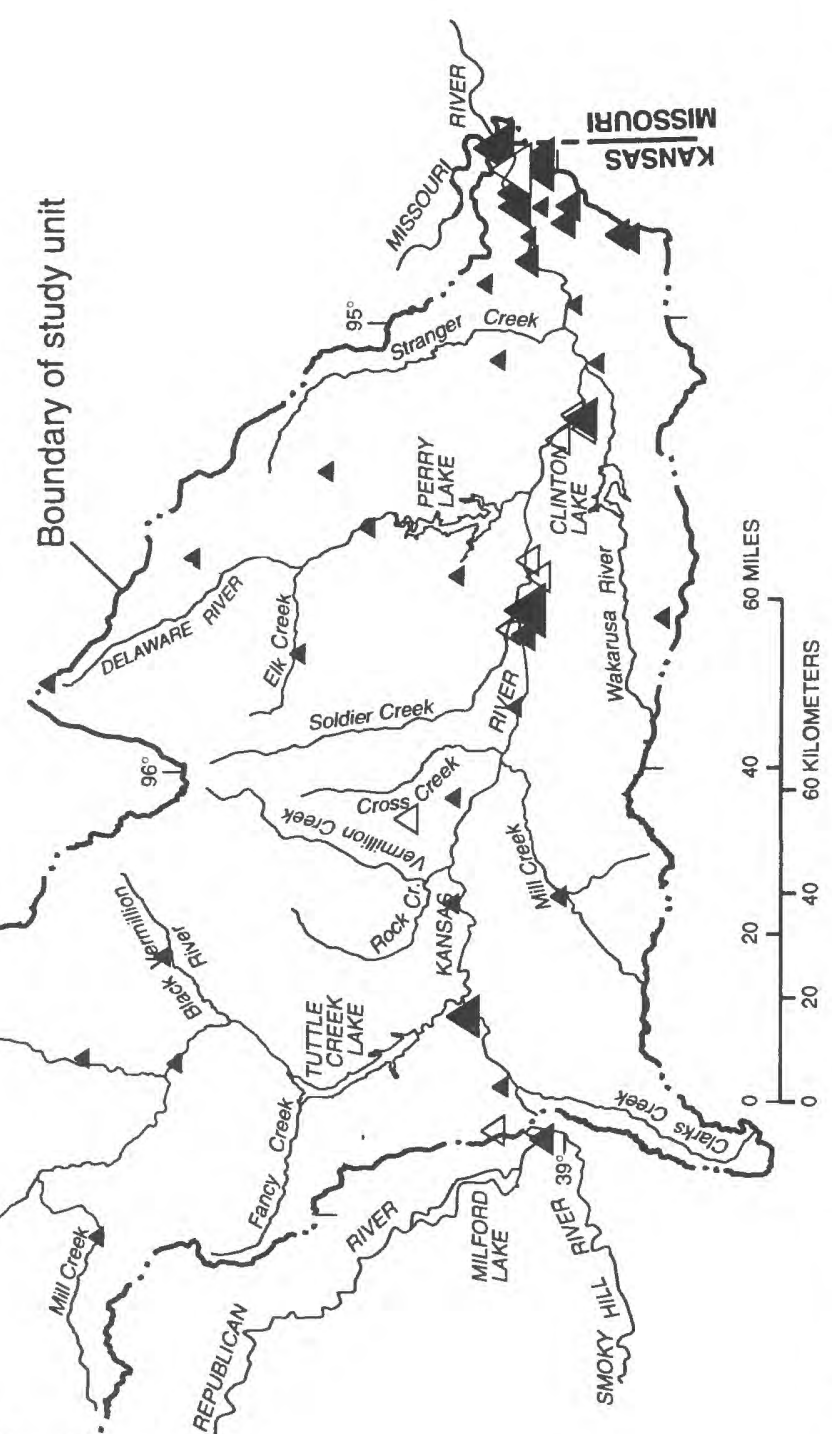

용

횽

힘

음

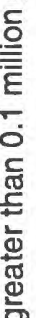

홍

总.

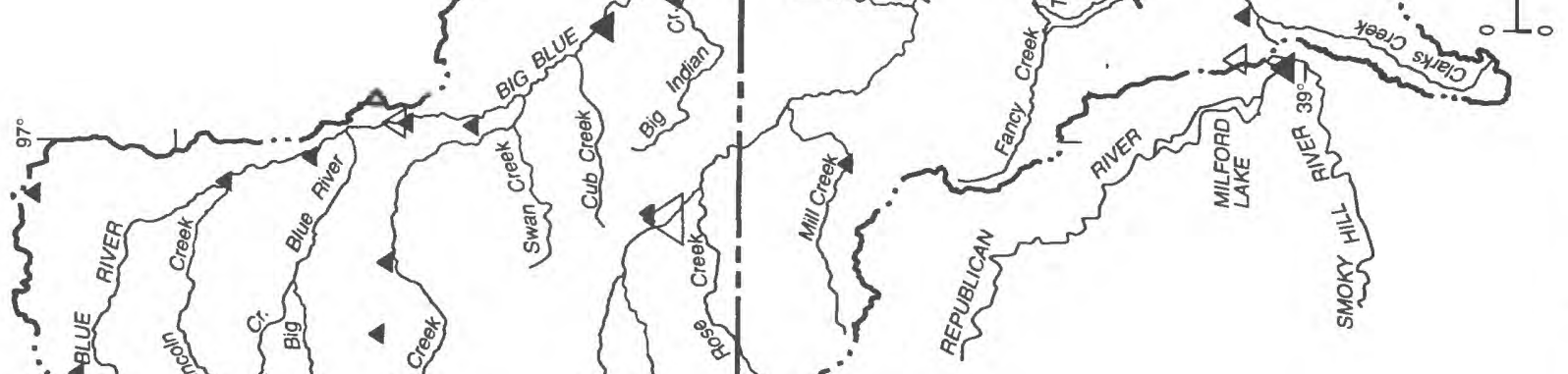
\{
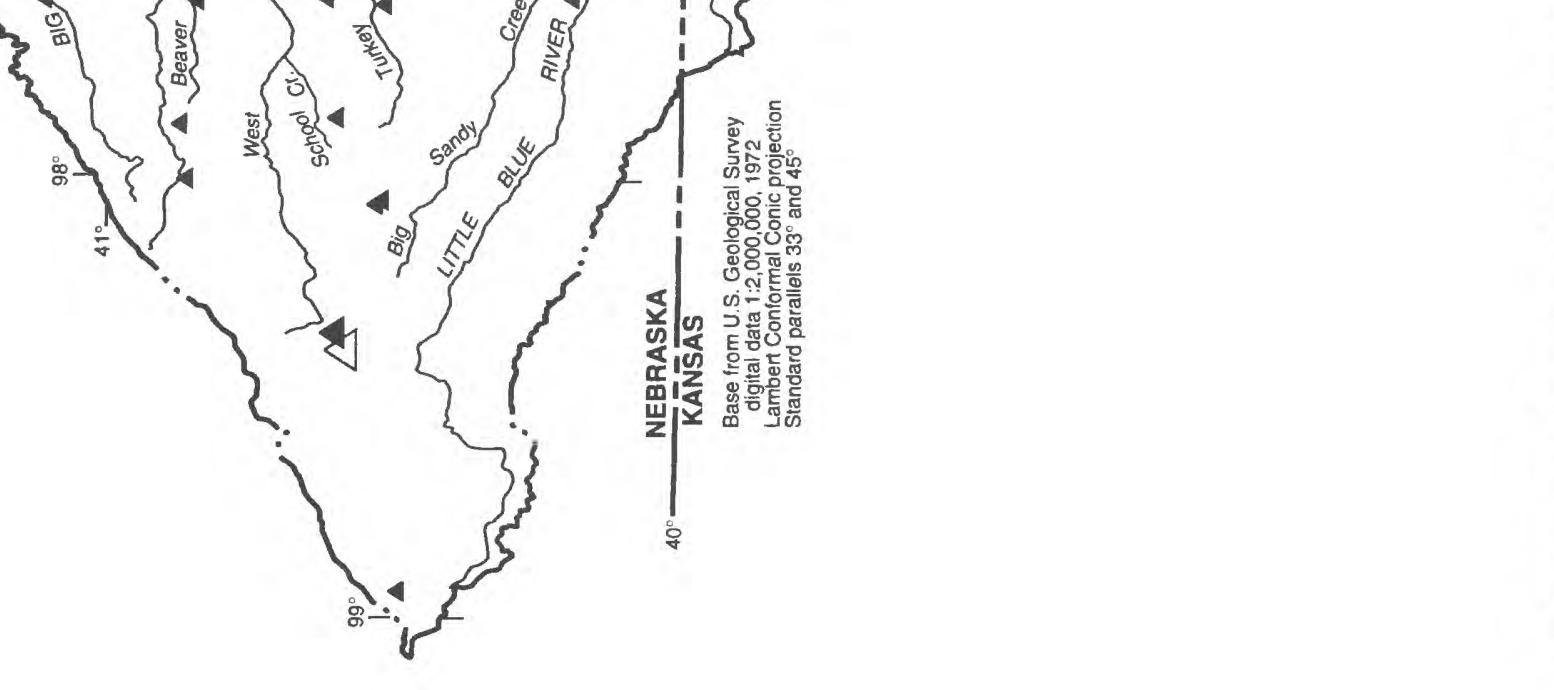

응

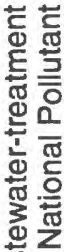

गे

증

공으 
surveys included onsite observations of streamflow, specific conductance, $\mathrm{pH}$, water temperature, and barometric pressure, and laboratory analyses for concentrations of nutrients, total organic carbon, chlorophyll, and suspended sediment.

\section{Sampling-Site Selection}

Synoptic-sampling sites were selected based on existing data, location of wastewater discharges, land use, and physiographic sections. Sites were selected to satisfy one or more of the following criteria: (1) include all regularly sampled stations; (2) locations where DO depletion or bacteria densities might be significant; (3) locations on streams draining areas with reasonably homogeneous land use; (4) sites near the confluence of major tributaries; and (5) additional sites on the main stem Kansas River and major tributary streams to provide a resolution of no more than 50 river mi between sites. A description of the 61 synoptic-sampling sites used in this study is provided in table 2. Location of these sites is shown in figure 10. The sites chosen for this study and corresponding map-index numbers are a subset of all 91 downstreamordered sampling sites for the lower Kansas River Basin NAWQA project as listed in Fallon and McChesney (1993); therefore, the map-index numbers for the 61 sites used in this study are not numbered consecutively but maintain consistency between individual reports for the lower Kansas River Basin NAWQA project.

\section{DISSOLVED-OXYGEN CONCENTRATIONS}

\section{Sources, Consumption, and Water-Quality Criteria}

Possible sources of DO in streams are: (1) ground water and surface runoff, (2) photosynthesis, and (3) physical aeration. Typically, ground water has very small concentrations of DO and, unless physically aerated prior to entering the stream, contributes little to the oxygen content of the receiving stream. Photosynthesis, the biochemical process of converting carbon dioxide and water into glucose and oxygen, is driven by light energy and carried on in the cells of all algae and plants containing chlorophyll. The oxygen produced in this process either becomes concentrated in cellular protoplasm or diffuses outward into the surrounding water. Factors that affect the contribution of photosynthesis to the DO concentration of a stream include light intensity, turbidity, algal population, nutrient concentration, and water temperature.

Physical aeration, the process of securing oxygen directly from the atmosphere can be a major source of DO to streams, but its effect may have great variability from one stream to another depending upon such factors as streamflow velocity, turbulence, channel morphology, water temperature, and atmospheric pressure. The significance of water temperature and atmospheric pressure lies in the inverse relation of oxygen solubility to temperature and the direct relation to atmospheric pressure (Reid and Wood, 1976, p. 210-213).

Consumption of DO in streams is mainly the result of microbial decomposition of organic and nitrogenous organic compounds; oxidation of reduced compounds of sulfur, nitrogen, and iron; and cellular respiration. Respiration, in its simplest form, is the reverse of photosynthesis; glucose and oxygen are converted to carbon dioxide and water with a release of energy equivalent to that provided by sunlight in fueling the photosynthetic reaction (Robbins and others, 1964, p. 57). The introduction of large quantities of biochemical oxygen-demanding material, such as that discharged from a wastewater-treatment facility, can severely affect a stream with inadequate DO reserves and may produce a DO depression within a stream segment. These depressions often result in DO concentrations that are less than the acceptable water-quality criteria.

Water-quality criteria for DO have been established by the USEPA (U.S. Environmental Protection Agency, 1986). The 1-day minimum, warmwater criteria are $5.0 \mathrm{mg} / \mathrm{L}$ (milligrams per liter) for early life stages, which include all embryonic and larval stages and all juvenile forms to 30 days following hatching, and $3.0 \mathrm{mg} / \mathrm{L}$ for all other life stages. The daily minimums were established to prevent acute mortality of sensitive species resulting from a lack of oxygen. The criteria were designed to prevent significant episodes of continuous or regularly recurring exposures to DO concentrations at or near the lethal threshold. 
Table 2. Description of synoptic-sampling sites in the lower Kansas River Basin

\begin{tabular}{ccc}
$\begin{array}{c}\text { Map-index } \\
\text { number } \\
\text { (fig. 10) }\end{array}$ & $\begin{array}{c}\text { U.S. Geological } \\
\text { Survey site } \\
\text { identification } \\
\text { number }\end{array}$ & Site name \\
\hline
\end{tabular}

\begin{tabular}{|c|c|c|}
\hline 1 & 06879100 & Kansas River at Fort Riley, Kans. \\
\hline 2 & 390255096435000 & Clarks Creek near Fort Riley, Kans. \\
\hline 5 & 06879820 & Kansas River at Manhattan, Kans. \\
\hline 6 & 06879900 & Big Blue River at Surprise, Nebr. \\
\hline 9 & 405221097582100 & Lincoln Creek near Aurora, Nebr. \\
\hline 10 & 405438097354800 & Lincoln Creek near York, Nebr. \\
\hline 11 & 06880000 & Lincoln Creek near Seward, Nebr. \\
\hline 12 & 06880500 & Big Blue River at Seward, Nebr. \\
\hline 16 & 403611098200600 & West Fork Big Blue River near Hastings, Nebr. \\
\hline 17 & 404247097580600 & West Fork Big Blue River near Stockham, Nebr. \\
\hline 18 & 403749097503400 & School Creek near Sutton, Nebr. \\
\hline 19 & 404327097354600 & West Fork Big Blue River near McCool Junction, Nebr. \\
\hline 21 & 405029097322100 & Beaver Creek near York, Nebr. \\
\hline 23 & 06880800 & West Fork Big Blue River near Dorchester, Nebr. \\
\hline 26 & 06881000 & Big Blue River near Crete, Nebr. \\
\hline 27 & 403304097311400 & Turkey Creek near Geneva, Nebr. \\
\hline 28 & 06881200 & Turkey Creek near Wilber, Nebr. \\
\hline 29 & 402348096591100 & Swan Creek near Dewitt, Nebr. \\
\hline 31 & 401730096500200 & Cub Creek near Beatrice, Nebr. \\
\hline 32 & 06881500 & Big Blue River at Beatrice, Nebr. \\
\hline 34 & 400632096401600 & Big Indian Creek near Wymore, Nebr. \\
\hline 35 & 06882000 & Big Blue River at Barneston, Nebr. \\
\hline 36 & 06882510 & Big Blue River at Marysville, Kans. \\
\hline 37 & 402726098240500 & Little Blue River near Hastings, Nebr. \\
\hline 38 & 06883000 & Little Blue River near Deweese, Nebr. \\
\hline 39 & 401243097433500 & Little Blue River near Deshler, Nebr. \\
\hline 40 & 06883570 & Little Blue River near Alexandria (Gilead), Nebr. \\
\hline 41 & 401826097451100 & Big Sandy Creek near Davenport, Nebr. \\
\hline 42 & 06883940 & Big Sandy Creek at Alexandria, Nebr. \\
\hline 44 & 06884000 & Little Blue River near Fairbury, Nebr. \\
\hline
\end{tabular}


Table 2. Description of synoptic-sampling sites in the lower Kansas River Basin-Continued

\begin{tabular}{|c|c|c|}
\hline $\begin{array}{c}\text { Map-index } \\
\text { number } \\
\text { (fig. 10) }\end{array}$ & $\begin{array}{l}\text { U.S. Geological } \\
\text { Survey site } \\
\text { identification } \\
\text { number }\end{array}$ & Site name \\
\hline 46 & 400359097101400 & Rose Creek near Fairbury, Nebr. \\
\hline 47 & 06884025 & Little Blue River at Hollenberg, Kans. \\
\hline 48 & 395513096561100 & Mill Creek near Hanover, Kans. \\
\hline 49 & 06884400 & Little Blue River near Barnes, Kans. \\
\hline 50 & 06885500 & Black Vermillion River near Frankfort, Kans. \\
\hline 51 & 06886500 & Fancy Creek at Winkler, Kans. \\
\hline 52 & 06887000 & Big Blue River near Manhattan, Kans. \\
\hline 55 & 392844096093500 & Vermillion Creek near Onaga, Kans. \\
\hline 56 & 06888030 & Vermillion Creek near Louisville, Kans. \\
\hline 57 & 06888300 & Rock Creek near Louisville, Kans. \\
\hline 58 & 06888350 & Kansas River near Belvue, Kans. \\
\hline 59 & 06888500 & Mill Creek near Paxico, Kans. \\
\hline 60 & 390820095571500 & Cross Creek at Rossville, Kans. \\
\hline 61 & 06888705 & Kansas River at Willard, Kans. \\
\hline 62 & 06889000 & Kansas River at Topeka, Kans. \\
\hline 63 & 06889160 & Soldier Creek near Circleville, Kans. \\
\hline 64 & 06889500 & Soldier Creek near Topeka, Kans. \\
\hline 71 & 394757095434300 & Delaware River near Fairview, Kans. \\
\hline 72 & 06890100 & Delaware River near Muscotah, Kans. \\
\hline 73 & 392823095362800 & Elk Creek near Larkinburg, Kans. \\
\hline 74 & 06890900 & Delaware River below Perry Dam, Kans. \\
\hline 76 & 06891000 & Kansas River at Lecompton, Kans. \\
\hline 77 & 06891080 & Kansas River at Lawrence, Kans. \\
\hline 78 & 06891100 & Kansas River at Eudora, Kans. \\
\hline 79 & 385329095353400 & Wakarusa River near Berryton, Kans. \\
\hline 83 & 06891500 & Wakarusa River near Lawrence, Kans. \\
\hline 86 & 06891850 & Stranger Creek at Easton, Kans. \\
\hline 87 & 06892000 & Stranger Creek near Tonganoxie, Kans. \\
\hline 88 & 06892350 & Kansas River at DeSoto, Kans. \\
\hline 90 & 06892940 & Turkey Creek at Kansas City, Kans. \\
\hline 91 & 06892950 & Kansas River at Kansas City, Kans. \\
\hline
\end{tabular}

${ }^{1}$ Map-index numbers from Fallon and McChesney (1993). 


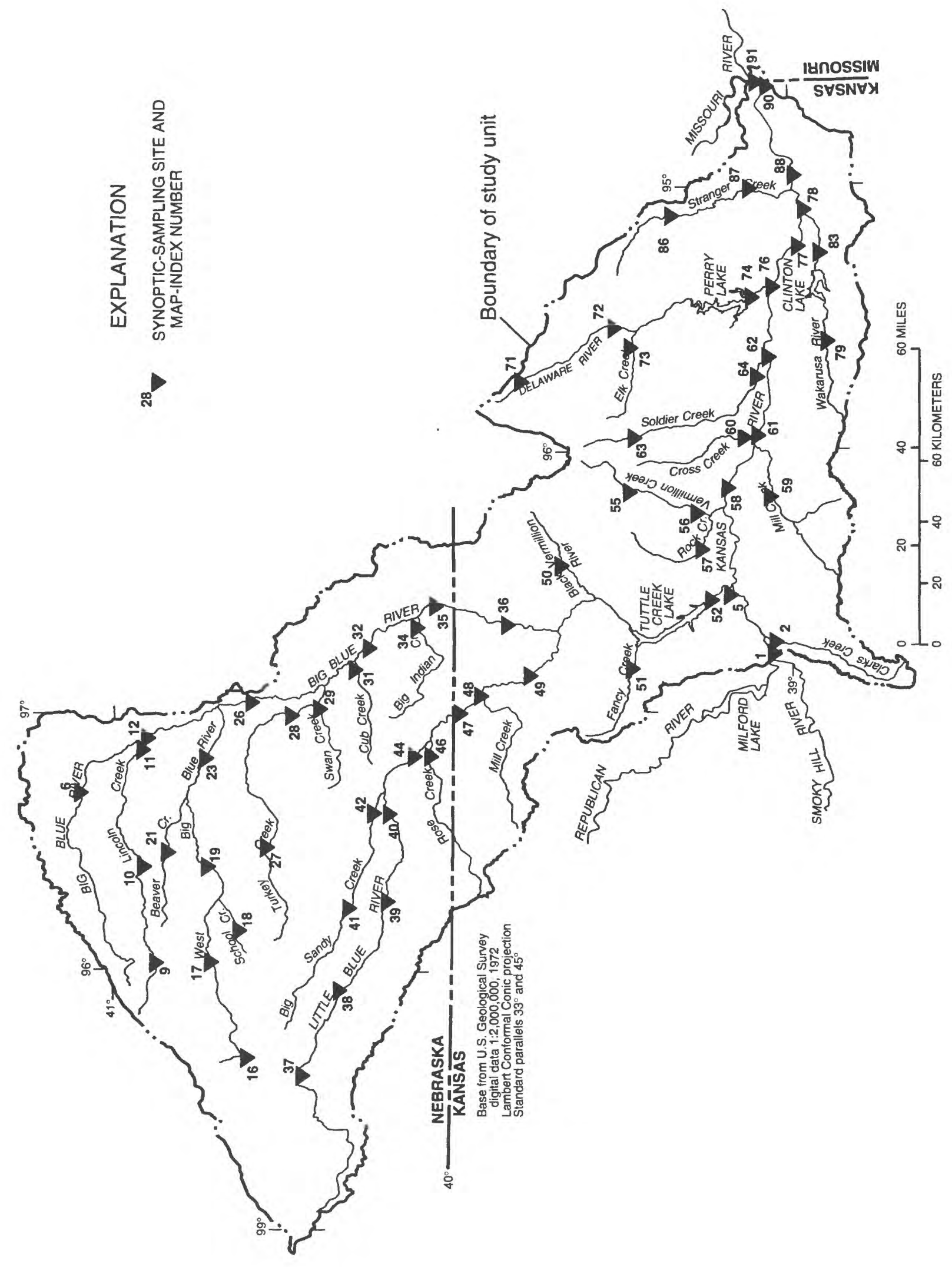

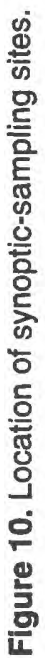




\section{Data Collection and Analysis}

Because of the diel variability of DO and to assess the streams at a time of minimal DO concentration, efforts were made to sample sites before sunrise. During the 6 days of the July 1988 synoptic survey, 31 sites were sampled under predawn conditions (0415 to 0635 hours). The remaining 30 sites generally were sampled at times that fit most efficiently into the sampling schedules. To define diel variability, 11 sites were selected to represent a range in drainage area, land use, and physiographic characteristics. These 11 sites were sampled at 4- or 6-hour intervals during a 24-hour period.

All determinations of DO were made onsite with portable meters equipped with DO-specific probes. The meters used for this investigation were Yellow Springs Instrument ${ }^{1}$ (YSI), model numbers 54 or 57 , with a YSI 5739 DO probe. These meters have a measurement range of 0.1 to $20.0 \mathrm{mg} / \mathrm{L}$. Before each measurement, the meters were calibrated using the air-calibration-in-water technique described in Hines and others (1977).

All samples for chemical analyses were collected in accordance with procedures described in Brown and others (1970). Generally, the equal-width-increment (EWI) method was used for all streams requiring depth-integrated sampling. A DH-81 suspendedsediment sampler was used for depth integration on wadeable streams. A D-77 cable-suspended sediment sampler was used from a bridge on streams where excessive depth or velocity prevented wading. On small streams where the use of a suspended-sediment sampler was impractical (shallow depth or small velocity), a dip sample at the centroid of flow was collected. Discrete dip or EWI samples collected during the horizontal transit of the stream were composited into a U.S. Geological Survey churn splitter. Representative subsamples for chemical analysis were withdrawn from the churn, bottled, and appropriately preserved.

Sample preservation and analysis of water samples for nutrients, organic carbon, and chlorophyll were made in accordance with procedures described in Fishman and Friedman (1989), Wershaw and others (1983), and Britton and Greeson (1987), respectively.

\footnotetext{
${ }^{1}$ The use of brand names in this report is for identification purposes only and does not constitute endorsement by the U.S. Geological Survey.
}

Chemical analysis of samples collected for this study was done by the U.S. Geological Survey laboratory in Arvada, Colo. Concentrations of dissolved chemical constituents presented in this report were determined for water samples that were filtered through a membrane filter with a pore size of 0.45 micrometer. Total concentrations of constituents were determined on unfiltered samples of the water-sediment mixture. Determinations made at the time of sample collection or at the U.S. Geological Survey laboratory, Lawrence, Kans., included streamflow discharge, specific conductance, $\mathrm{pH}$, water temperature, barometric pressure, and concentrations of DO and suspended sediment.

Streamflow discharge for water samples collected at established U.S. Geological Survey gaging stations with a stable, well-defined stream stage-discharge relation was determined by referencing the observed stream stage to the stage-discharge relation.

Streamflow discharge at gaging stations with a shifting or questionable stage-discharge relation and at ungaged sites was determined by measuring the streamflow at the time of sampling in accordance with procedures described in Buchanan and Somers (1976).

Onsite measurements of specific conductance and $\mathrm{pH}$ were made with portable electronic meters on an aliquot of the composite stream sample in accordance with procedures described in Fishman and Friedman (1989). All $\mathrm{pH}$ measurements were recorded to the nearest 0.1 standard unit. Water temperature was determined onsite with a mercury or alcohol thermometer and recorded to the nearest $0.5^{\circ} \mathrm{C}$. Barometric pressure was measured with a portable barometer calibrated to National Oceanic and Atmospheric Administration weather-station readings prior to start of the survey. Barometric pressure was recorded to the nearest 1.0 millimeter of mercury. Determinations of suspended-sediment concentrations were made in accordance with procedures described in Guy (1977).

Results of water-quality determinations and DO concentrations at all synoptic-sampling sites are shown in table 3. Results of analyses of water samples for concentrations of nutrients, total organic carbon, chlorophyll, and suspended sediment are shown in table 8 in the "Supplemental Information" section at the end of this report. Included in tables 3 and 8 are results from synoptic surveys of July 1988, November 1988, March 1989, and May-June 1989. Also included in tables 3 and 8 are quality-assurance measurements and analyses. These measurements and analyses consist of duplicate determinations at 
Table 3. Results of water-quality determinations and dissolved-oxygen concentrations at synoptic-sampling sites in the lower Kansas River Basin, July 1988 through June 1989

$\left[\mu \mathrm{S} / \mathrm{cm}\right.$, microsiemens per centimeter at 25 degrees Celsius; ${ }^{\circ} \mathrm{C}$, degrees Celsius; $\mathrm{mm}$ of $\mathrm{Hg}$, millimeters of mercury; $\mathrm{mg} / \mathrm{L}$, milligrams per liter, E, estimated; --, no data; >, greater than]

\begin{tabular}{|c|c|c|c|c|c|c|c|c|c|}
\hline $\begin{array}{l}\text { Site map- } \\
\text { index } \\
\text { number } \\
\text { (fig. 10) }\end{array}$ & Date & $\begin{array}{c}\text { Time } \\
\text { (24-hour) }\end{array}$ & $\begin{array}{l}\text { Stream- } \\
\text { flow, } \\
\text { instan- } \\
\text { taneous } \\
\text { (cubic } \\
\text { feet per } \\
\text { second) }\end{array}$ & $\begin{array}{c}\text { Specific } \\
\text { conduct- } \\
\text { ance } \\
(\mu \mathrm{S} / \mathrm{cm})\end{array}$ & $\begin{array}{c}\text { pH } \\
\text { (standard } \\
\text { units) }\end{array}$ & $\begin{array}{l}\text { Temper- } \\
\text { ature, } \\
\text { water }\left({ }^{\circ} \mathrm{C}\right)\end{array}$ & $\begin{array}{c}\text { Baro- } \\
\text { metric } \\
\text { pressure } \\
\text { (mm of } \\
\mathrm{Hg})\end{array}$ & $\begin{array}{l}\text { Oxygen, } \\
\text { dissolved } \\
\text { (mg/L) }\end{array}$ & $\begin{array}{c}\text { Oxygen, } \\
\text { dissolved } \\
\text { (percent } \\
\text { satur- } \\
\text { ation) }\end{array}$ \\
\hline \multirow[t]{4}{*}{1} & $07-27-88$ & 1300 & 353 & 1,830 & 8.4 & 28.0 & 739 & 9.3 & 123 \\
\hline & $11-15-88$ & 1205 & 327 & 2,600 & 8.3 & 15.0 & 718 & 10.2 & 108 \\
\hline & 03-08-89 & 1000 & 395 & 1,730 & 8.5 & 3.0 & 745 & 15.3 & 117 \\
\hline & $06-01-89$ & 1010 & 500 & 1,250 & 8.0 & 21.0 & 740 & 8.6 & 100 \\
\hline \multirow[t]{4}{*}{2} & $07-27-88$ & 1150 & .65 & 571 & 8.3 & 27.5 & 740 & 8.1 & 106 \\
\hline & $11-15-88$ & 1050 & 2.1 & 613 & 8.3 & 15.0 & 720 & 8.8 & 93 \\
\hline & 03-08-89 & 1140 & 3.7 & 543 & 8.4 & 5.5 & 745 & 14.1 & 115 \\
\hline & 06-01-89 & 0830 & 1.3 & 586 & 8.2 & 22.0 & 738 & 6.0 & 71 \\
\hline \multirow[t]{4}{*}{5} & $07-24-88$ & 1020 & 240 & 1,290 & 8.1 & 26.0 & 738 & 7.8 & 100 \\
\hline & $11-16-88$ & 0850 & 455 & 1,940 & 8.7 & 7.5 & 731 & 10.6 & 93 \\
\hline & 03-09-89 & 0930 & 395 & 1,580 & 8.6 & 6.0 & 749 & 15.2 & 125 \\
\hline & $06-02-89$ & 0820 & 496 & 1,090 & 8.6 & 21.0 & 739 & 9.8 & 114 \\
\hline \multirow[t]{4}{*}{6} & $07-26-88$ & 0730 & 6.2 & 583 & 8.2 & 24.0 & 741 & 7.0 & 86 \\
\hline & $11-14-88$ & 1200 & 1.4 & 656 & 8.0 & 6.0 & 718 & 11.0 & 94 \\
\hline & 03-08-89 & 1015 & 24 & 334 & 7.8 & .5 & 730 & 8.3 & 60 \\
\hline & $05-31-89$ & 1445 & E1.5 & 657 & 8.2 & 19.5 & 726 & 11.2 & 128 \\
\hline \multirow[t]{6}{*}{9} & $07-26-88$ & 0945 & .34 & 458 & 7.6 & 20.5 & 725 & 1.6 & 19 \\
\hline & $07-26-88$ & 2150 & E. 30 & -- & -- & 26.0 & 722. & 2.9 & 38 \\
\hline & $07-27-88$ & 0550 & E.30 & -- & -- & 21.5 & 721 & 1.2 & 14 \\
\hline & $11-15-88$ & 0815 & 1.4 & 1,130 & 7.8 & 6.5 & 705 & 4.4 & 39 \\
\hline & $03-07-89$ & 1015 & E.01 & 452 & 5.9 & .5 & 773 & 2.0 & 14 \\
\hline & $05-31-89$ & 0930 & .40 & 964 & 7.5 & 16.0 & 718 & 1.0 & 11 \\
\hline \multirow[t]{5}{*}{10} & $07-26-88$ & 0900 & 8.2 & 570 & 7.9 & 21.0 & 734 & 6.3 & 74 \\
\hline & $07-26-88$ & 1300 & 8.2 & 570 & 7.7 & 23.0 & 725 & 7.0 & 86 \\
\hline & $07-26-88$ & 1700 & 8.2 & 574 & 7.8 & 27.0 & 725 & 7.6 & 101 \\
\hline & $07-26-88$ & 2100 & 8.2 & 549 & 7.8 & 28.0 & 724 & 7.4 & 100 \\
\hline & $07-27-88$ & 0100 & 8.2 & 552 & 7.9 & 26.0 & 724 & 6.2 & 81 \\
\hline
\end{tabular}


Table 3. Results of water-quality determinations and dissolved-oxygen concentrations at synoptic-sampling sites in the lower Kansas River Basin, July 1988 through June 1989-Continued

\begin{tabular}{|c|c|c|c|c|c|c|c|c|c|}
\hline $\begin{array}{l}\text { Site map- } \\
\text { index } \\
\text { number } \\
\text { (flg. 10) }\end{array}$ & Date & $\begin{array}{c}\text { Time } \\
\text { (24-hour) }\end{array}$ & $\begin{array}{l}\text { Stream- } \\
\text { flow, } \\
\text { instan- } \\
\text { taneous } \\
\text { (cubic } \\
\text { feet per } \\
\text { second) }\end{array}$ & $\begin{array}{c}\text { Specific } \\
\text { conduct- } \\
\text { ance } \\
(\mu \mathrm{S} / \mathrm{cm})\end{array}$ & $\begin{array}{c}\text { pH } \\
\text { (standard } \\
\text { units) }\end{array}$ & $\begin{array}{c}\text { Temper- } \\
\text { ature, } \\
\text { water }\left({ }^{\circ} \mathrm{C}\right)\end{array}$ & $\begin{array}{c}\text { Baro- } \\
\text { metric } \\
\text { pressure } \\
\text { (mm of } \\
\mathbf{H g})\end{array}$ & $\begin{array}{c}\text { Oxygen, } \\
\text { dissolved } \\
(\mathrm{mg} / \mathrm{L})\end{array}$ & $\begin{array}{c}\text { Oxygen, } \\
\text { dissolved } \\
\text { (percent } \\
\text { satur- } \\
\text { ation) }\end{array}$ \\
\hline \multirow[t]{5}{*}{10} & $07-27-88$ & 0500 & 8.2 & 561 & 7.7 & 23.0 & 724 & 5.5 & 68 \\
\hline & $07-27-88$ & 0900 & 8.2 & 566 & 7.9 & 22.0 & 725 & 6.0 & 72 \\
\hline & $11-14-88$ & 1345 & -- & 513 & 8.0 & 7.0 & 715 & 10.4 & 91 \\
\hline & 03-07-89 & 1000 & .88 & 680 & 7.6 & 0 & 735 & -- & -- \\
\hline & $05-31-89$ & 1330 & .19 & 586 & 7.6 & 16.5 & 724 & 6.1 & 66 \\
\hline \multirow[t]{4}{*}{11} & $07-26-88$ & 0845 & 30 & 580 & 8.1 & 23.0 & 742 & 6.7 & 80 \\
\hline & $11-14-88$ & 1050 & 17 & 621 & 8.3 & 6.0 & 723 & 10.9 & 92 \\
\hline & 03-08-89 & 1215 & 25 & 408 & 7.8 & .5 & 739 & 11.5 & 82 \\
\hline & $05-31-89$ & 1600 & E17 & 641 & 8.1 & 18.5 & 727 & 8.3 & 93 \\
\hline \multirow[t]{9}{*}{12} & $07-26-88$ & 1000 & 55 & 555 & 8.0 & 23.0 & 741 & 5.8 & 70 \\
\hline & $07-26-88$ & 1005 & 55 & 555 & 8.0 & 23.0 & 741 & 5.8 & 70 \\
\hline & $07-26-88$ & 1400 & 55 & 548 & 8.0 & 25.0 & 741 & 7.4 & 92 \\
\hline & $07-26-88$ & 1800 & 55 & 549 & 8.2 & 26.0 & 741 & 8.4 & 107 \\
\hline & $07-26-88$ & 2200 & 55 & 585 & 7.6 & 26.0 & 740 & 7.4 & 94 \\
\hline & $07-27-88$ & 0200 & 55 & 577 & 8.0 & 24.0 & 740 & 6.7 & 82 \\
\hline & $07-27-88$ & 0600 & 55 & 595 & 8.0 & 23.0 & 741 & 5.5 & 66 \\
\hline & $11-14-88$ & 0845 & 29 & 689 & 8.2 & 5.5 & 724 & 11.4 & 95 \\
\hline & 03-08-89 & 1130 & 114 & 476 & 7.6 & .5 & 740 & 12.2 & 87 \\
\hline \multirow[t]{7}{*}{16} & $07-26-88$ & 0630 & 6.7 & -- & 7.6 & 21.0 & 719 & 2.1 & 25 \\
\hline & $07-26-88$ & 1800 & E6.7 & -- & -- & 25.5 & 721 & 4.8 & 63 \\
\hline & $07-27-88$ & 1215 & E6.7 & -- & -- & 20.5 & 720 & 2.1 & 25 \\
\hline & $09-06-88$ & 1145 & 5.8 & 946 & 7.7 & 20.0 & 714 & 2.5 & 29 \\
\hline & $11-15-88$ & 1015 & 3.0 & 1,030 & 7.5 & 14.5 & 702 & .6 & 6 \\
\hline & $03-07-89$ & 1600 & 6.4 & 909 & 7.7 & 11.5 & 717 & 4.0 & 40 \\
\hline & $05-30-89$ & 1145 & 4.4 & 838 & 7.6 & 21.5 & 716 & 4.9 & 59 \\
\hline \multirow[t]{5}{*}{17} & $07-25-88$ & 1230 & 14 & -- & 8.0 & 26.5 & 726 & 6.8 & 92 \\
\hline & $07-26-88$ & 1300 & E14 & 532 & 7.8 & 24.5 & 726 & 7.1 & 90 \\
\hline & $07-26-88$ & 1700 & E14 & 356 & 8.0 & 27.0 & 725 & 8.1 & 107 \\
\hline & $07-26-88$ & 2100 & E18 & 535 & 8.0 & 27.0 & 728 & 6.5 & 86 \\
\hline & $07-27-88$ & 0100 & E18 & 644 & 8.0 & 25.5 & 723 & 5.6 & 72 \\
\hline
\end{tabular}


Table 3. Results of water-quality determinations and dissolved-oxygen concentrations at synoptic-sampling sites in the lower Kansas River Basin, July 1988 through June 1989-Continued

\begin{tabular}{|c|c|c|c|c|c|c|c|c|c|}
\hline $\begin{array}{l}\text { Site map- } \\
\text { index } \\
\text { number } \\
\text { (fig. 10) }\end{array}$ & Date & $\begin{array}{c}\text { Time } \\
\text { (24-hour) }\end{array}$ & $\begin{array}{l}\text { Stream- } \\
\text { flow, } \\
\text { instan- } \\
\text { taneous } \\
\text { (cubic } \\
\text { feet per } \\
\text { second) }\end{array}$ & $\begin{array}{c}\text { Specific } \\
\text { conduct- } \\
\text { ance } \\
(\mu \mathrm{S} / \mathrm{cm})\end{array}$ & $\begin{array}{c}\text { pH } \\
\text { (standard } \\
\text { units) }\end{array}$ & $\begin{array}{l}\text { Temper- } \\
\text { ature, } \\
\text { water }\left({ }^{\circ} \mathrm{C}\right)\end{array}$ & $\begin{array}{c}\text { Baro- } \\
\text { metric } \\
\text { pressure } \\
(\mathrm{mm} \text { of } \\
\mathrm{Hg})\end{array}$ & $\begin{array}{l}\text { Oxygen, } \\
\text { dissolved } \\
\text { (mg/L) }\end{array}$ & $\begin{array}{c}\text { Oxygen, } \\
\text { dissolved } \\
\text { (percent } \\
\text { satur- } \\
\text { ation) }\end{array}$ \\
\hline \multirow[t]{5}{*}{17} & $07-27-88$ & 0500 & E18 & 662 & 8.0 & 23.5 & 722 & 5.9 & 74 \\
\hline & $07-27-88$ & 0900 & E18 & 639 & 7.9 & 22.5 & 724 & 6.0 & 73 \\
\hline & $07-27-88$ & 1300 & E16 & 626 & 7.8 & 24.5 & 725 & 7.1 & 90 \\
\hline & $11-15-88$ & 1130 & 3.5 & 970 & 7.7 & 6.5 & 708 & 8.4 & 74 \\
\hline & $03-07-89$ & 1300 & 2.6 & 920 & 7.6 & .5 & 724 & 9.2 & 67 \\
\hline \multirow[t]{4}{*}{18} & $07-25-88$ & 1130 & 3.8 & 427 & 7.8 & 23.5 & 727 & 5.1 & 63 \\
\hline & $11-15-88$ & 1230 & .26 & 998 & 7.8 & 7.5 & 707 & 8.9 & 80 \\
\hline & 03-07-89 & 1445 & .55 & 828 & 7.7 & 0 & 724 & 12.4 & 90 \\
\hline & 05-30-89 & 1445 & .26 & 1,060 & 8.5 & 26.5 & 717 & 12.1 & 161 \\
\hline \multirow[t]{4}{*}{19} & $07-25-88$ & 1200 & 27 & 533 & 8.3 & 27.0 & 734 & 7.6 & 99 \\
\hline & $11-15-88$ & 1300 & 14 & 713 & 8.3 & 7.5 & 709 & 11.2 & 101 \\
\hline & 03-07-89 & 1245 & 15 & 630 & 7.6 & 0 & 732 & 14.7 & 105 \\
\hline & $05-31-89$ & 1100 & 15 & 604 & 7.8 & 19.0 & 723 & 6.2 & 71 \\
\hline \multirow[t]{5}{*}{21} & $07-26-88$ & 1010 & 6.5 & 660 & 7.9 & 23.0 & 734 & 7.1 & 86 \\
\hline & 09-06-88 & 1440 & 2.5 & 995 & 8.3 & 19.5 & 721 & 11.1 & 128 \\
\hline & $11-14-88$ & 1425 & 2.5 & 980 & 8.3 & 8.5 & 716 & 17.8 & 162 \\
\hline & 03-07-89 & 1145 & 2.9 & 852 & 7.4 & 0 & 737 & -- & -- \\
\hline & $05-31-89$ & 1245 & 2.2 & 870 & 7.6 & 18.5 & 724 & 4.9 & 56 \\
\hline \multirow[t]{14}{*}{23} & $07-25-88$ & 1245 & 75 & 518 & 8.2 & 25.5 & 743 & 7.6 & 95 \\
\hline & $08-08-88$ & 1115 & 81 & 556 & 8.1 & 26.0 & 726 & 7.6 & 99 \\
\hline & $08-31-88$ & 1400 & 48 & 590 & 8.5 & 21.0 & 730 & 11.3 & 133 \\
\hline & $10-12-88$ & 1230 & 55 & 467 & 7.8 & 11.5 & 733 & 10.6 & 101 \\
\hline & $11-07-88$ & 1200 & 57 & -- & 8.3 & 8.0 & 730 & 11.3 & -- \\
\hline & $11-15-88$ & 1420 & 58 & 610 & 8.0 & 8.0 & 711 & 9.9 & 90 \\
\hline & $12-06-88$ & 1000 & 53 & -- & 8.2 & 3.5 & 735 & 10.7 & -- \\
\hline & $01-03-89$ & 1130 & 59 & 684 & 7.8 & 0 & 732 & 13.8 & 99 \\
\hline & $02-07-89$ & 1000 & 49 & 668 & 7.8 & 0 & 731 & 10.8 & 77 \\
\hline & $03-07-89$ & 1400 & 76 & 539 & 8.0 & 0 & 731 & 13.1 & 94 \\
\hline & $03-08-89$ & 1345 & 98 & 484 & 7.9 & 1.0 & 741 & 13.6 & 99 \\
\hline & $04-04-89$ & 0955 & 70 & 632 & 8.1 & 7.0 & 726 & 12.7 & 110 \\
\hline & $05-02-89$ & 0830 & 64 & 609 & 8.3 & 9.5 & 726 & 9.7 & 89 \\
\hline & $05-31-89$ & 1430 & E54 & 610 & 8.2 & 19.0 & 725 & 5.2 & 59 \\
\hline
\end{tabular}


Table 3. Results of water-quality determinations and dissolved-oxygen concentrations at synoptic-sampling sites in the lower Kansas River Basin, July 1988 through June 1989-Continued

\begin{tabular}{|c|c|c|c|c|c|c|c|c|c|}
\hline $\begin{array}{c}\text { Site map- } \\
\text { index } \\
\text { number } \\
\text { (fig. 10) }\end{array}$ & Date & $\begin{array}{c}\text { Time } \\
\text { (24-hour) }\end{array}$ & $\begin{array}{l}\text { Stream- } \\
\text { flow, } \\
\text { instan- } \\
\text { taneous } \\
\text { (cubic } \\
\text { feet per } \\
\text { second) }\end{array}$ & $\begin{array}{c}\text { Specific } \\
\text { conduct- } \\
\text { ance } \\
(\mu \mathrm{S} / \mathrm{cm})\end{array}$ & $\begin{array}{c}\text { pH } \\
\text { (standard } \\
\text { units) }\end{array}$ & $\begin{array}{l}\text { Temper- } \\
\text { ature, } \\
\text { water }\left({ }^{\circ} \mathrm{C}\right)\end{array}$ & $\begin{array}{c}\text { Baro- } \\
\text { metric } \\
\text { pressure } \\
(\mathbf{m m} \text { of } \\
\mathbf{H g})\end{array}$ & $\begin{array}{l}\text { Oxygen, } \\
\text { dissolved } \\
\text { (mg/L) }\end{array}$ & $\begin{array}{c}\text { Oxygen, } \\
\text { dissolved } \\
\text { (percent } \\
\text { satur- } \\
\text { ation) }\end{array}$ \\
\hline \multirow[t]{5}{*}{26} & $07-25-88$ & 1030 & 184 & 526 & 8.6 & 25.5 & 744 & 8.8 & 110 \\
\hline & $09-07-88$ & 0840 & 83 & 644 & 8.4 & 16.0 & 720 & 8.2 & 88 \\
\hline & $11-14-88$ & 1530 & 112 & 715 & 8.2 & 8.5 & 722 & 13.5 & 122 \\
\hline & 03-08-89 & 1500 & 192 & 515 & 7.9 & 4.0 & 741 & 11.6 & 91 \\
\hline & $05-31-89$ & 1300 & E164 & 628 & 8.3 & 20.0 & 726 & 7.7 & 89 \\
\hline \multirow[t]{4}{*}{27} & $07-25-88$ & 1325 & 2.1 & 362 & 8.0 & 28.5 & 734 & 6.8 & 91 \\
\hline & $11-15-88$ & 1110 & .29 & 476 & 7.8 & 8.5 & 711 & 10.0 & 92 \\
\hline & 03-07-89 & 1345 & 1.5 & 290 & 7.8 & .5 & 730 & -- & -- \\
\hline & $05-30-89$ & 1445 & E.50 & 860 & 8.6 & 29.5 & 715 & 17.5 & 246 \\
\hline \multirow[t]{4}{*}{28} & $07-25-88$ & 0600 & 22 & 436 & 7.0 & 24.5 & 734 & 6.6 & 82 \\
\hline & $11-14-88$ & 1445 & 7.2 & 862 & 7.7 & 8.0 & 722 & 9.1 & 81 \\
\hline & 03-07-89 & 0855 & 6.8 & 722 & 7.5 & .5 & 736 & 10.3 & 74 \\
\hline & $05-31-89$ & 1215 & E10 & 695 & 8.0 & 19.5 & 726 & 6.9 & 79 \\
\hline \multirow[t]{4}{*}{29} & $07-25-88$ & 0630 & 13 & 847 & 7.5 & 24.0 & 734 & 6.3 & 78 \\
\hline & $11-14-88$ & 1200 & 11 & 1,000 & 7.8 & 8.5 & 725 & 12.6 & 114 \\
\hline & 03-07-89 & 1240 & 30 & 739 & 7.8 & 1.0 & 736 & 13.4 & 98 \\
\hline & $05-31-89$ & 1100 & 8.0 & 913 & 8.0 & 19.0 & 725 & 9.2 & 105 \\
\hline \multirow[t]{9}{*}{31} & $07-25-88$ & 1330 & 3.6 & 441 & 7.6 & 27.0 & 760 & 8.4 & 106 \\
\hline & $07-25-88$ & 1335 & 3.6 & 441 & 7.6 & 27.0 & 760 & 8.4 & 106 \\
\hline & $07-25-88$ & 1730 & 3.6 & 439 & 7.9 & 26.0 & 740 & 8.2 & 104 \\
\hline & $07-25-88$ & 2100 & 3.6 & 440 & 7.6 & 26.5 & 738 & 7.4 & 95 \\
\hline & $07-26-88$ & 0200 & 3.6 & 402 & 8.0 & 23.0 & 734 & 6.6 & 80 \\
\hline & $07-26-88$ & 0510 & 3.6 & 411 & 8.1 & 21.5 & 736 & 6.1 & 72 \\
\hline & $07-26-88$ & 0915 & 3.6 & 462 & 8.1 & 21.0 & 734 & 7.0 & 82 \\
\hline & $11-14-88$ & 1045 & .75 & 601 & 7.2 & 7.0 & 728 & 8.4 & 73 \\
\hline & 03-08-89 & 0900 & 2.9 & 518 & 7.6 & .5 & 739 & 9.3 & 67 \\
\hline 31 & $05-30-89$ & 1630 & 0.51 & 582 & 8.0 & 24.0 & 722 & 5.1 & 64 \\
\hline
\end{tabular}


Table 3. Results of water-quality determinations and dissolved-oxygen concentrations at synoptic-sampling sites in the lower Kansas River Basin, July 1988 through June 1989-Continued

\begin{tabular}{|c|c|c|c|c|c|c|c|c|c|}
\hline $\begin{array}{c}\text { Site map- } \\
\text { index } \\
\text { number } \\
\text { (fig. 10) }\end{array}$ & Date & $\begin{array}{c}\text { Time } \\
\text { (24-hour) }\end{array}$ & $\begin{array}{l}\text { Stream- } \\
\text { flow, } \\
\text { instan- } \\
\text { taneous } \\
\text { (cubic } \\
\text { feet per } \\
\text { second) }\end{array}$ & $\begin{array}{c}\text { Specific } \\
\text { conduct- } \\
\text { ance } \\
(\mu \mathrm{S} / \mathrm{cm})\end{array}$ & $\begin{array}{c}\text { pH } \\
\text { (standard } \\
\text { units) }\end{array}$ & $\begin{array}{l}\text { Temper- } \\
\text { ature, } \\
\text { water }\left({ }^{\circ} \mathrm{C}\right)\end{array}$ & $\begin{array}{c}\text { Baro- } \\
\text { metric } \\
\text { pressure } \\
\text { (mm of } \\
\mathrm{Hg})\end{array}$ & $\begin{array}{l}\text { Oxygen, } \\
\text { dissolved } \\
\text { (mg/L) }\end{array}$ & $\begin{array}{c}\text { Oxygen, } \\
\text { dissolved } \\
\text { (percent } \\
\text { satur- } \\
\text { ation) }\end{array}$ \\
\hline \multirow[t]{10}{*}{32} & $07-25-88$ & 1330 & 322 & 533 & 8.4 & 28.0 & 761 & 7.3 & 94 \\
\hline & $07-25-88$ & 1700 & 318 & 529 & 8.3 & 29.0 & 735 & 7.2 & 97 \\
\hline & $07-25-88$ & 2100 & 314 & 520 & 8.4 & 29.0 & 735 & 6.7 & 91 \\
\hline & $07-26-88$ & 0115 & 299 & 550 & 8.3 & 27.0 & 734 & 6.7 & 88 \\
\hline & $07-26-88$ & 0950 & 280 & 567 & 8.3 & 25.0 & 734 & 7.2 & 91 \\
\hline & $07-27-88$ & 0510 & 247 & 530 & 8.5 & 26.0 & 734 & 5.8 & 74 \\
\hline & $11-14-88$ & 0900 & 192 & 801 & 7.9 & 7.0 & 729 & 11.2 & 97 \\
\hline & $11-14-88$ & 0905 & 192 & 801 & 7.9 & 7.0 & 728 & 11.2 & 97 \\
\hline & $11-14-88$ & 1300 & 192 & 808 & 8.2 & 8.0 & 726 & 12.7 & 113 \\
\hline & $11-14-88$ & 1305 & 192 & 808 & 8.2 & 8.0 & 726 & 12.7 & 113 \\
\hline & $11-14-88$ & 1700 & 188 & 845 & 8.2 & 8.0 & 724 & 12.3 & 110 \\
\hline & $11-14-88$ & 1705 & 188 & 845 & 8.2 & 8.0 & 724 & 12.3 & 110 \\
\hline & $11-14-88$ & 2100 & 188 & 776 & 8.2 & 8.5 & 723 & 11.6 & 105 \\
\hline & $11-14-88$ & 2105 & 188 & 776 & 8.2 & 8.5 & 723 & 11.6 & 105 \\
\hline & $11-15-88$ & 0100 & 185 & 764 & 8.2 & 9.0 & 720 & 11.2 & 103 \\
\hline & $11-15-88$ & 0105 & 185 & 764 & 8.2 & 9.0 & 720 & 11.2 & 103 \\
\hline & $11-15-88$ & 0500 & 185 & 759 & 8.1 & 9.5 & 718 & 10.4 & 97 \\
\hline & $11-15-88$ & 0505 & 185 & 759 & 8.1 & 9.5 & 718 & 10.4 & 97 \\
\hline & $11-15-88$ & 0900 & 192 & 761 & 8.1 & 9.5 & 718 & 10.1 & 94 \\
\hline & $11-15-88$ & 0905 & 192 & 716 & 8.1 & 9.5 & 718 & 10.1 & 94 \\
\hline & 03-07-89 & 1000 & 237 & 592 & 7.9 & 0 & 740 & 12.8 & 90 \\
\hline & 03-07-89 & 1005 & 237 & 592 & 7.9 & 0 & 740 & 12.8 & 90 \\
\hline & 03-07-89 & 1400 & 237 & 594 & 7.9 & 1.0 & 737 & 13.1 & 95 \\
\hline & 03-07-89 & 1405 & 237 & 594 & 7.9 & 1.0 & 737 & 13.1 & 95 \\
\hline & $03-07-89$ & 1800 & 237 & 583 & 7.8 & .5 & 736 & 12.7 & 91 \\
\hline & 03-07-89 & 1805 & 237 & 583 & 7.8 & .5 & 736 & 12.7 & 91 \\
\hline & 03-07-89 & 2200 & 237 & 570 & 7.7 & .5 & 737 & 14.0 & 101 \\
\hline & 03-07-89 & 2205 & 237 & 570 & 7.7 & .5 & 737 & 14.0 & 101 \\
\hline & 03-08-89 & 0200 & 237 & 537 & 7.8 & .5 & 738 & 13.0 & 93 \\
\hline & 03-08-89 & 0205 & 237 & 537 & 7.8 & .5 & 738 & 13.0 & 93 \\
\hline
\end{tabular}


Table 3. Results of water-quality determinations and dissolved-oxygen concentrations at synoptic-sampling sites in the lower Kansas River Basin, July 1988 through June 1989-Continued

\begin{tabular}{|c|c|c|c|c|c|c|c|c|c|}
\hline $\begin{array}{l}\text { Site map- } \\
\text { index } \\
\text { number } \\
\text { (fig. 10) }\end{array}$ & Date & $\begin{array}{c}\text { Time } \\
\text { (24-hour) }\end{array}$ & $\begin{array}{l}\text { Stream- } \\
\text { flow, } \\
\text { instan- } \\
\text { taneous } \\
\text { (cubic } \\
\text { feet per } \\
\text { second) }\end{array}$ & $\begin{array}{c}\text { Specific } \\
\text { conduct- } \\
\text { ance } \\
(\mu \mathrm{S} / \mathrm{cm})\end{array}$ & $\begin{array}{c}\text { pH } \\
\text { (standard } \\
\text { units) }\end{array}$ & $\begin{array}{l}\text { Temper- } \\
\text { ature, } \\
\text { water }\left({ }^{\circ} \mathrm{C}\right)\end{array}$ & $\begin{array}{c}\text { Baro- } \\
\text { metric } \\
\text { pressure } \\
\text { (mm of } \\
\mathrm{Hg})\end{array}$ & $\begin{array}{l}\text { Oxygen, } \\
\text { dissolved } \\
\text { (mg/L) }\end{array}$ & $\begin{array}{c}\text { Oxygen, } \\
\text { dissolved } \\
\text { (percent } \\
\text { satur- } \\
\text { ation) }\end{array}$ \\
\hline \multirow[t]{18}{*}{32} & $03-08-89$ & 0600 & 237 & 548 & 7.9 & 0 & 739 & 12.0 & 85 \\
\hline & 03-08-89 & 0605 & 237 & 548 & 7.9 & 0 & 739 & 12.0 & 85 \\
\hline & 03-08-89 & 1000 & 237 & 574 & 7.9 & .5 & 739 & 13.2 & 95 \\
\hline & 03-08-89 & 1005 & 237 & 574 & 7.9 & .5 & 739 & 13.2 & 95 \\
\hline & $05-30-89$ & 1000 & 163 & 740 & 8.6 & 23.0 & 722 & 7.7 & 95 \\
\hline & $05-30-89$ & 1005 & 163 & 740 & 8.6 & 23.0 & 722 & 7.7 & 95 \\
\hline & $05-30-89$ & 1500 & 163 & 735 & 8.9 & 25.0 & 722 & 13.9 & 178 \\
\hline & $05-30-89$ & 1505 & 163 & 735 & 8.9 & 25.0 & 722 & 13.9 & 178 \\
\hline & $05-30-89$ & 1800 & 163 & 708 & 8.9 & 25.0 & 723 & 13.5 & 173 \\
\hline & $05-30-89$ & 1810 & 163 & 708 & 8.9 & 25.0 & 723 & 13.5 & 173 \\
\hline & $05-30-89$ & 2200 & 163 & 720 & 8.8 & 25.0 & 725 & 10.2 & 130 \\
\hline & $05-30-89$ & 2205 & 163 & 720 & 8.8 & 25.0 & 725 & 10.2 & 130 \\
\hline & $05-31-89$ & 0200 & 163 & 725 & 8.3 & 24.5 & 726 & 8.1 & 102 \\
\hline & $05-31-89$ & 0210 & 163 & 725 & 8.3 & 24.5 & 726 & 8.1 & 102 \\
\hline & $05-31-89$ & 0600 & 163 & 731 & 8.8 & 22.5 & 727 & 6.0 & 73 \\
\hline & $05-31-89$ & 0610 & 163 & 731 & 8.8 & 22.5 & 727 & 6.0 & 73 \\
\hline & $05-31-89$ & 1000 & 163 & 735 & 8.8 & 21.5 & 725 & 7.1 & 85 \\
\hline & $05-31-89$ & 1005 & 163 & 735 & 8.8 & 21.5 & 725 & 7.1 & 85 \\
\hline \multirow[t]{12}{*}{34} & $07-25-88$ & 0650 & 12 & -- & 8.0 & 25.0 & 740 & 6.2 & 78 \\
\hline & $07-26-88$ & 0915 & 19 & 448 & 8.1 & 24.0 & 738 & 6.4 & 79 \\
\hline & $07-26-88$ & 1045 & 19 & 475 & 7.8 & 24.5 & 738 & 7.2 & 89 \\
\hline & $07-26-88$ & 1445 & 19 & 476 & 8.0 & 28.0 & 735 & 8.9 & 118 \\
\hline & $07-26-88$ & 1450 & 19 & 476 & 8.0 & 28.0 & 735 & 8.9 & 118 \\
\hline & $07-26-88$ & 1845 & 19 & 490 & 8.1 & 28.0 & 733 & 9.3 & 124 \\
\hline & $07-26-88$ & 2230 & 19 & 546 & 8.1 & 25.0 & 734 & 7.5 & 95 \\
\hline & $07-27-88$ & 0230 & 19 & 517 & 7.9 & 24.5 & 732 & 7.5 & 94 \\
\hline & $07-27-88$ & 0635 & 19 & 500 & 8.0 & 23.0 & 730 & 7.3 & 89 \\
\hline & $11-14-88$ & 1020 & 5.2 & 1,060 & 7.8 & 6.0 & 727 & 10.1 & 85 \\
\hline & 03-07-89 & 1520 & 4.5 & 811 & 8.3 & 2.5 & 738 & 18.9 & 143 \\
\hline & $05-30-89$ & 1345 & 3.1 & 930 & 8.3 & 27.0 & 723 & 8.1 & 108 \\
\hline
\end{tabular}


Table 3. Results of water-quality determinations and dissolved-oxygen concentrations at synoptic-sampling sites in the lower Kansas River Basin, July 1988 through June 1989-Continued

\begin{tabular}{|c|c|c|c|c|c|c|c|c|c|}
\hline $\begin{array}{l}\text { Site map- } \\
\text { index } \\
\text { number } \\
\text { (fig. 10) }\end{array}$ & Date & $\begin{array}{c}\text { Time } \\
\text { (24-hour) }\end{array}$ & $\begin{array}{l}\text { Stream- } \\
\text { flow, } \\
\text { instan- } \\
\text { taneous } \\
\text { (cubic } \\
\text { feet per } \\
\text { second) }\end{array}$ & $\begin{array}{c}\text { Specific } \\
\text { conduct- } \\
\text { ance } \\
(\mu \mathrm{S} / \mathrm{cm})\end{array}$ & $\begin{array}{c}\text { pH } \\
\text { (standard } \\
\text { units) }\end{array}$ & $\begin{array}{l}\text { Temper- } \\
\text { ature, } \\
\text { water }\left({ }^{\circ} \mathrm{C}\right)\end{array}$ & $\begin{array}{c}\text { Baro- } \\
\text { metric } \\
\text { pressure } \\
(\mathrm{mm} \text { of } \\
\mathrm{Hg})\end{array}$ & $\begin{array}{l}\text { Oxygen, } \\
\text { dissolved } \\
\text { (mg/L) }\end{array}$ & $\begin{array}{c}\text { Oxygen, } \\
\text { dissolved } \\
\text { (percent } \\
\text { satur- } \\
\text { atlon) }\end{array}$ \\
\hline \multirow[t]{14}{*}{35} & $07-25-88$ & 0540 & 376 & 524 & 8.2 & 25.0 & 740 & 7.4 & 93 \\
\hline & $08-09-88$ & 0915 & 138 & 654 & 8.6 & 28.0 & 734 & 6.6 & 88 \\
\hline & $08-30-88$ & 1400 & 136 & 552 & 8.6 & 23.5 & 739 & 8.3 & 101 \\
\hline & $10-18-88$ & 1000 & 171 & 554 & 7.8 & 15.0 & 741 & 9.3 & 95 \\
\hline & $11-08-88$ & 1200 & 166 & 738 & 8.8 & 9.0 & 740 & 12.3 & 110 \\
\hline & $11-14-88$ & 1145 & 193 & 755 & 8.2 & 6.5 & 726 & 13.0 & 111 \\
\hline & $12-06-88$ & 0900 & 229 & 750 & 8.7 & 3.5 & 734 & 15.6 & 122 \\
\hline & 01-04-89 & 1100 & 200 & 809 & 8.8 & 1.5 & 734 & 17.5 & 130 \\
\hline & $01-31-89$ & 1100 & 329 & 779 & 7.4 & 1.0 & 718 & 15.9 & 119 \\
\hline & 03-07-89 & 1435 & 303 & 593 & 8.2 & 1.0 & 739 & 14.4 & 105 \\
\hline & 03-28-89 & 1130 & 270 & 689 & 8.3 & 12.5 & 726 & 11.0 & 109 \\
\hline & $04-19-89$ & 1130 & 223 & 699 & 8.9 & 13.0 & 738 & 10.0 & 98 \\
\hline & $05-24-89$ & 0940 & 220 & 628 & 8.6 & 23.0 & 715 & 7.8 & 97 \\
\hline & $05-30-89$ & 1245 & 187 & 665 & 8.4 & 23.0 & 723 & 8.6 & 106 \\
\hline \multirow[t]{4}{*}{36} & $07-25-88$ & 1230 & 393 & 557 & 7.8 & 28.0 & 737 & -- & - \\
\hline & $11-16-88$ & 1050 & 270 & 792 & 7.6 & 7.5 & 741 & 12.1 & 104 \\
\hline & 03-09-89 & 0940 & 316 & 585 & 8.4 & .5 & 741 & 14.7 & 105 \\
\hline & $05-31-89$ & 1240 & 161 & 661 & 9.0 & 25.0 & 730 & 10.8 & 137 \\
\hline \multirow[t]{4}{*}{37} & $07-26-88$ & 0515 & 18 & 349 & 7.8 & 22.5 & 727 & 7.8 & 95 \\
\hline & $11-15-88$ & 1100 & 8.0 & 380 & 7.6 & 7.5 & 703 & 10.9 & 99 \\
\hline & $03-08-89$ & 0800 & 11 & 461 & 7.6 & 4.0 & 722 & 11.9 & 96 \\
\hline & $05-30-89$ & 1200 & 8.3 & 476 & 8.5 & 20.0 & 714 & 11.7 & 138 \\
\hline \multirow[t]{4}{*}{38} & $07-26-88$ & 0810 & 31 & 412 & 8.0 & 20.0 & 738 & 7.9 & 90 \\
\hline & $11-15-88$ & 0940 & 55 & 370 & 7.5 & 7.5 & 706 & 10.9 & 98 \\
\hline & $03-08-89$ & 0920 & 67 & 444 & 8.1 & 1.5 & 726 & 13.7 & 103 \\
\hline & $05-30-89$ & 1320 & 52 & 477 & 8.3 & 24.0 & 714 & 8.6 & 109 \\
\hline \multirow[t]{4}{*}{39} & $07-26-88$ & 0630 & 32 & 375 & 8.3 & 21.0 & 738 & 7.3 & 85 \\
\hline & $11-15-88$ & 0815 & 52 & 370 & 8.0 & 7.5 & 709 & 10.2 & 92 \\
\hline & $03-08-89$ & 1100 & 79 & 444 & 8.1 & 0 & 730 & 12.5 & 89 \\
\hline & $05-31-89$ & 1030 & 45 & 495 & 8.7 & 19.0 & 722 & 7.8 & 89 \\
\hline
\end{tabular}


Table 3. Results of water-quality determinations and dissolved-oxygen concentrations at synoptic-sampling sites in the lower Kansas River Basin, July 1988 through June 1989-Continued

\begin{tabular}{|c|c|c|c|c|c|c|c|c|c|}
\hline $\begin{array}{l}\text { Site map- } \\
\text { index } \\
\text { number } \\
\text { (fig. 10) }\end{array}$ & Date & $\begin{array}{c}\text { Time } \\
\text { (24-hour) }\end{array}$ & $\begin{array}{l}\text { Stream- } \\
\text { flow, } \\
\text { instan- } \\
\text { taneous } \\
\text { (cubic } \\
\text { feet per } \\
\text { second) }\end{array}$ & $\begin{array}{c}\text { Specific } \\
\text { conduct- } \\
\text { ance } \\
(\mu \mathrm{S} / \mathrm{cm})\end{array}$ & $\begin{array}{c}\text { pH } \\
\text { (standard } \\
\text { units) }\end{array}$ & $\begin{array}{c}\text { Temper- } \\
\text { ature, } \\
\text { water }\left({ }^{\circ} \mathrm{C}\right)\end{array}$ & $\begin{array}{c}\text { Baro- } \\
\text { metric } \\
\text { pressure } \\
\text { (mm of } \\
\mathrm{Hg})\end{array}$ & $\begin{array}{l}\text { Oxygen, } \\
\text { dissolved } \\
\text { (mg/L) }\end{array}$ & $\begin{array}{c}\text { Oxygen, } \\
\text { dissolved } \\
\text { (percent } \\
\text { satur- } \\
\text { ation) }\end{array}$ \\
\hline \multirow[t]{4}{*}{40} & $07-26-88$ & 0630 & 55 & 397 & 8.0 & 22.0 & 734 & 6.0 & 71 \\
\hline & $11-14-88$ & 1655 & 69 & 430 & 8.2 & 9.0 & 718 & 12.2 & 112 \\
\hline & 03-08-89 & 1345 & 118 & 448 & 8.2 & 0 & 734 & 16.4 & 117 \\
\hline & $05-31-89$ & 1200 & 61 & 500 & 8.3 & 20.0 & 730 & 8.5 & 98 \\
\hline 41 & $07-26-88$ & 0600 & 1.5 & 378 & 7.8 & 18.0 & 738 & 8.1 & 89 \\
\hline \multirow[t]{4}{*}{42} & $07-26-88$ & 0545 & 37 & 370 & 7.5 & 17.5 & 734 & 6.6 & 72 \\
\hline & $11-14-88$ & 1730 & 20 & 337 & 7.4 & 11.0 & 716 & 7.9 & 76 \\
\hline & 03-08-89 & 1250 & 26 & 354 & 7.7 & 12.5 & 734 & 11.3 & 110 \\
\hline & $05-31-89$ & 1130 & 25 & 385 & 7.8 & 15.0 & 725 & 9.8 & 102 \\
\hline \multirow[t]{9}{*}{44} & $07-25-88$ & 0845 & 133 & 396 & 7.4 & 28.0 & 734 & 6.8 & 91 \\
\hline & $07-26-88$ & 1320 & 119 & 374 & -- & 27.0 & 732 & 9.4 & 123 \\
\hline & $07-26-88$ & 1710 & 127 & 423 & - & 27.0 & 733 & 10.6 & 139 \\
\hline & $07-26-88$ & 2150 & 116 & 435 & -- & 27.0 & 734 & 6.5 & 85 \\
\hline & $07-27-88$ & 0120 & 127 & 375 & - & 27.5 & 732 & 5.7 & 75 \\
\hline & $07-27-88$ & 0545 & 112 & 393 & 8.3 & 25.0 & 734 & 5.3 & 67 \\
\hline & $11-14-88$ & 1555 & 118 & 455 & 8.0 & 7.0 & 720 & 13.1 & 114 \\
\hline & $03-08-89$ & 1225 & 133 & 488 & 8.2 & 2.5 & 738 & 15.0 & 114 \\
\hline & $05-31-89$ & 1300 & 91 & 520 & 8.2 & 21.0 & 730 & 8.3 & 97 \\
\hline \multirow[t]{9}{*}{46} & $07-25-88$ & 0615 & 18 & 398 & 7.8 & 26.0 & 734 & 6.4 & 82 \\
\hline & $07-26-88$ & 1355 & 9.0 & 411 & 8.1 & 26.5 & 732 & 7.0 & 91 \\
\hline & $07-26-88$ & 1750 & E8.5 & 460 & 7.9 & 27.0 & 732 & 7.2 & 94 \\
\hline & $07-26-88$ & 2110 & 8.0 & 498 & 7.7 & 26.5 & 734 & 5.6 & 73 \\
\hline & $07-27-88$ & 0100 & E7.5 & 440 & 8.2 & 25.5 & 732 & 7.0 & 89 \\
\hline & $07-27-88$ & 0510 & E7.0 & 448 & 7.2 & 23.5 & 734 & 5.6 & 69 \\
\hline & $11-14-88$ & 1515 & 8.0 & 560 & 8.1 & 6.5 & 723 & 12.9 & 111 \\
\hline & 03-08-89 & 1200 & 13 & 599 & 8.1 & 1.0 & 738 & 15.5 & 113 \\
\hline & $05-31-89$ & 1330 & 6.8 & 604 & 8.0 & 21.0 & 730 & 6.9 & 81 \\
\hline \multirow[t]{3}{*}{47} & $07-25-88$ & 1150 & 163 & 501 & 8.7 & 29.0 & 740 & 7.2 & 97 \\
\hline & $07-27-88$ & 0920 & 119 & 512 & 9.0 & 27.0 & 740 & 15.0 & 195 \\
\hline & $08-09-88$ & 1135 & 78 & 578 & 8.4 & 26.5 & 733 & 10.9 & 141 \\
\hline
\end{tabular}


Table 3. Results of water-quality determinations and dissolved-oxygen concentrations at synoptic-sampling sites in the lower Kansas River Basin, July 1988 through June 1989-Continued

\begin{tabular}{|c|c|c|c|c|c|c|c|c|c|}
\hline $\begin{array}{l}\text { Site map- } \\
\text { index } \\
\text { number } \\
\text { (fig. 10) }\end{array}$ & Date & $\begin{array}{c}\text { Time } \\
\text { (24-hour) }\end{array}$ & $\begin{array}{l}\text { Stream- } \\
\text { flow, } \\
\text { instan- } \\
\text { taneous } \\
\text { (cubic } \\
\text { feet per } \\
\text { second) }\end{array}$ & $\begin{array}{c}\text { Specific } \\
\text { conduct- } \\
\text { ance } \\
(\mu \mathrm{S} / \mathrm{cm})\end{array}$ & $\begin{array}{c}\text { pH } \\
\text { (standard } \\
\text { units) }\end{array}$ & $\begin{array}{l}\text { Temper- } \\
\text { ature, } \\
\text { water }\left({ }^{\circ} \mathrm{C}\right)\end{array}$ & $\begin{array}{c}\text { Baro- } \\
\text { metric } \\
\text { pressure } \\
\text { ( } \mathrm{mm} \text { of } \\
\mathrm{Hg} \text { ) }\end{array}$ & $\begin{array}{l}\text { Oxygen, } \\
\text { dissolved } \\
\text { (mg/L) }\end{array}$ & $\begin{array}{c}\text { Oxygen, } \\
\text { dissolved } \\
\text { (percent } \\
\text { satur- } \\
\text { ation) }\end{array}$ \\
\hline \multirow[t]{12}{*}{47} & $08-30-88$ & 1100 & 75 & 610 & 8.6 & 21.0 & 739 & 10.3 & 119 \\
\hline & $10-18-88$ & 1300 & 113 & 518 & 8.0 & 14.0 & 739 & 9.5 & 95 \\
\hline & $11-09-88$ & 1200 & 118 & 520 & 8.3 & 8.0 & 737 & 10.4 & 91 \\
\hline & $11-14-88$ & 1350 & 133 & 523 & 8.1 & 9.0 & 722 & 12.6 & 115 \\
\hline & $12-06-88$ & 1300 & 135 & 564 & 8.3 & 3.0 & 733 & 14.5 & 112 \\
\hline & $01-10-89$ & 1050 & 86 & 696 & 8.2 & 0 & 733 & 13.1 & 93 \\
\hline & $02-07-89$ & 1115 & 109 & 643 & 7.4 & 0 & 747 & -- & -- \\
\hline & 03-07-89 & 1100 & 179 & 537 & 8.1 & 0 & 737 & 13.4 & 95 \\
\hline & $03-08-89$ & 1040 & 200 & 524 & 8.1 & .5 & 740 & 14.1 & 101 \\
\hline & $04-05-89$ & 0955 & 138 & 566 & 8.3 & 9.5 & 736 & 13.3 & 121 \\
\hline & $05-01-89$ & 1200 & 142 & 593 & 8.4 & 12.5 & 738 & 10.7 & 104 \\
\hline & $05-31-89$ & 1420 & 110 & 600 & 8.4 & 21.0 & 730 & 8.7 & 102 \\
\hline \multirow[t]{4}{*}{48} & $07-26-88$ & 1215 & 12 & 763 & 8.3 & 24.0 & 734 & 7.0 & 87 \\
\hline & $11-16-88$ & 1400 & 12 & 855 & 8.1 & 7.0 & 732 & 8.5 & 73 \\
\hline & $03-09-89$ & 0830 & 13 & 652 & 8.2 & 1.0 & 738 & 13.7 & 100 \\
\hline & $05-31-89$ & 1415 & 5.9 & 734 & 8.3 & 23.0 & 730 & 7.3 & 89 \\
\hline \multirow[t]{11}{*}{49} & $07-25-88$ & 1400 & 206 & 454 & 7.9 & 31.0 & 737 & 10.6 & 148 \\
\hline & $11-16-88$ & 1130 & 166 & 580 & 8.5 & 5.0 & 735 & 12.4 & 101 \\
\hline & $11-16-88$ & 1135 & 166 & 580 & 8.5 & 5.0 & 735 & 12.4 & 101 \\
\hline & $03-08-89$ & 1630 & 150 & 715 & 8.3 & 4.5 & 738 & 12.0 & 96 \\
\hline & $03-08-89$ & 1635 & 150 & 715 & 8.3 & 4.5 & 738 & 12.0 & 96 \\
\hline & 03-08-89 & 1715 & 150 & 715 & 8.3 & 4.5 & 738 & 12.0 & 96 \\
\hline & 03-08-89 & 1720 & 150 & 715 & 8.3 & 4.5 & 738 & 12.0 & 96 \\
\hline & $05-31-89$ & 1540 & 110 & 640 & 8.8 & 24.0 & 730 & 11.8 & 147 \\
\hline & $05-31-89$ & 1545 & 110 & 640 & 8.8 & 24.0 & 730 & 11.8 & 147 \\
\hline & $05-31-89$ & 1550 & 110 & 639 & 8.8 & 24.0 & 730 & 11.8 & 147 \\
\hline & $05-31-89$ & 1555 & 110 & 639 & 8.8 & 24.0 & 730 & 11.8 & 147 \\
\hline \multirow[t]{4}{*}{50} & $07-24-88$ & 1320 & 7.0 & -- & 8.1 & 26.0 & 736 & 7.9 & 101 \\
\hline & $11-16-88$ & 1340 & 16 & 572 & 7.9 & 8.0 & 740 & 10.8 & 94 \\
\hline & $11-16-88$ & 1350 & 16 & 572 & 7.9 & 8.0 & 740 & 10.8 & 94 \\
\hline & $11-16-88$ & 1355 & 16 & 572 & 7.9 & 8.0 & 740 & 10.8 & 94 \\
\hline
\end{tabular}


Table 3. Results of water-quality determinations and dissolved-oxygen concentrations at synoptic-sampling sites in the lower Kansas River Basin, July 1988 through June 1989-Continued

\begin{tabular}{|c|c|c|c|c|c|c|c|c|c|}
\hline $\begin{array}{c}\text { Site map- } \\
\text { index } \\
\text { number } \\
\text { (fig. 10) }\end{array}$ & Date & $\begin{array}{c}\text { Time } \\
\text { (24-hour) }\end{array}$ & $\begin{array}{l}\text { Stream- } \\
\text { flow, } \\
\text { instan- } \\
\text { taneous } \\
\text { (cubic } \\
\text { feet per } \\
\text { second) }\end{array}$ & $\begin{array}{c}\text { Specific } \\
\text { conduct- } \\
\text { ance } \\
(\mu \mathrm{S} / \mathrm{cm})\end{array}$ & $\begin{array}{c}\text { pH } \\
\text { (standard } \\
\text { units) }\end{array}$ & $\begin{array}{c}\text { Temper- } \\
\text { ature, } \\
\text { water }\left({ }^{\circ} \mathrm{C}\right)\end{array}$ & $\begin{array}{c}\text { Baro- } \\
\text { metric } \\
\text { pressure } \\
(\mathrm{mm} \text { of } \\
\mathrm{Hg})\end{array}$ & $\begin{array}{l}\text { Oxygen, } \\
\text { dissolved } \\
\text { (mg/L) }\end{array}$ & $\begin{array}{c}\text { Oxygen, } \\
\text { dissolved } \\
\text { (percent } \\
\text { satur- } \\
\text { ation) }\end{array}$ \\
\hline \multirow[t]{2}{*}{50} & $03-09-89$ & 1130 & 16 & 588 & 8.3 & 3.0 & 740 & 16.8 & 129 \\
\hline & $05-31-89$ & 1055 & 4.0 & 592 & 8.1 & 21.0 & 732 & 13.5 & 158 \\
\hline \multirow[t]{4}{*}{51} & $07-24-88$ & 1445 & 5.7 & 655 & 8.2 & 29.0 & 738 & 8.6 & 116 \\
\hline & $11-16-88$ & 0940 & 15 & 857 & 8.9 & 8.0 & 737 & 8.6 & 75 \\
\hline & 03-09-89 & 1115 & 6.0 & 1,350 & 8.3 & 6.0 & 740 & 15.4 & 128 \\
\hline & $05-31-89$ & 1730 & 1.0 & 1,110 & 8.1 & 25.0 & 730 & 6.3 & 80 \\
\hline \multirow[t]{4}{*}{52} & $07-24-88$ & 1045 & 1,870 & 549 & 8.1 & 26.0 & 738 & 10.0 & 128 \\
\hline & $11-15-88$ & 1445 & 492 & 660 & 8.2 & 12.0 & 719 & 11.2 & 110 \\
\hline & 03-08-89 & 1430 & 442 & 684 & 8.2 & 4.0 & 746 & 18.3 & 143 \\
\hline & $06-02-89$ & 1030 & 396 & 591 & 8.3 & 21.0 & 738 & 7.9 & 92 \\
\hline \multirow[t]{4}{*}{55} & $07-27-88$ & 0930 & 1.4 & 504 & 6.6 & 23.0 & 740 & 5.4 & 65 \\
\hline & $11-15-88$ & 1330 & 1.6 & 865 & 7.8 & 12.0 & 720 & 8.4 & 83 \\
\hline & $03-08-89$ & 1345 & 4.3 & 526 & 8.4 & 1.0 & 743 & 17.2 & 124 \\
\hline & $05-31-89$ & 0900 & .59 & 503 & 8.0 & 23.5 & 733 & 12.2 & 150 \\
\hline \multirow[t]{4}{*}{56} & $07-27-88$ & 1120 & 4.2 & 550 & 7.1 & 27.0 & 742 & 9.0 & 116 \\
\hline & $11-15-88$ & 1115 & 3.6 & - & 7.7 & 10.0 & 725 & 8.6 & - \\
\hline & 03-08-89 & 1100 & 6.2 & 629 & 8.3 & 3.0 & 746 & 16.8 & 128 \\
\hline & $06-01-89$ & 1450 & 1.6 & 468 & 8.3 & 22.5 & 742 & 10.4 & 124 \\
\hline \multirow[t]{3}{*}{57} & $07-29-88$ & 0815 & 2.2 & 457 & 7.9 & 20.0 & 739 & 6.8 & 77 \\
\hline & $03-08-89$ & 1645 & 2.4 & 637 & 8.4 & 8.0 & 742 & 13.6 & 118 \\
\hline & $06-01-89$ & 1645 & 1.7 & 476 & 7.7 & 18.0 & 740 & 8.5 & 93 \\
\hline \multirow[t]{4}{*}{58} & $07-27-88$ & 0910 & 2,550 & 701 & 8.4 & 25.0 & 730 & 7.7 & 98 \\
\hline & $11-15-88$ & 1445 & 1,190 & 1,040 & 8.4 & 17.0 & 715 & 8.4 & 93 \\
\hline & $03-10-89$ & 1110 & 897 & 1,080 & 8.5 & 8.0 & 742 & 11.0 & 96 \\
\hline & $05-30-89$ & 1845 & 1,040 & 845 & 8.3 & 28.0 & 730 & 7.0 & 94 \\
\hline \multirow[t]{4}{*}{59} & $07-27-88$ & 1010 & 7.8 & 656 & 8.1 & 26.0 & 730 & 6.8 & 88 \\
\hline & $11-15-88$ & 1100 & 5.7 & 698 & 8.0 & 13.5 & 721 & 8.7 & 88 \\
\hline & 03-08-89 & 1310 & 4.6 & - & 8.3 & 8.0 & 750 & 11.8 & -- \\
\hline & $06-01-89$ & 1145 & 2.7 & 727 & 7.9 & 21.5 & 742 & 5.7 & 67 \\
\hline
\end{tabular}


Table 3. Results of water-quality determinations and dissolved-oxygen concentrations at synoptic-sampling sites in the lower Kansas River Basin, July 1988 through June 1989-Continued

\begin{tabular}{|c|c|c|c|c|c|c|c|c|c|}
\hline $\begin{array}{l}\text { Site map- } \\
\text { index } \\
\text { number } \\
\text { (fig. 10) }\end{array}$ & Date & $\begin{array}{c}\text { Time } \\
\text { (24-hour) }\end{array}$ & $\begin{array}{l}\text { Stream- } \\
\text { flow, } \\
\text { instan- } \\
\text { taneous } \\
\text { (cubic } \\
\text { feet per } \\
\text { second) }\end{array}$ & $\begin{array}{c}\text { Specific } \\
\text { conduct- } \\
\text { ance } \\
(\mu \mathrm{S} / \mathrm{cm})\end{array}$ & $\begin{array}{c}\text { pH } \\
\text { (standard } \\
\text { units) }\end{array}$ & $\begin{array}{l}\text { Temper- } \\
\text { ature, } \\
\text { water }\left({ }^{\circ} \mathrm{C}\right)\end{array}$ & $\begin{array}{c}\text { Baro- } \\
\text { metric } \\
\text { pressure } \\
\text { (mm of } \\
\mathrm{Hg})\end{array}$ & $\begin{array}{l}\text { Oxygen, } \\
\text { dissolved } \\
\text { (mg/L) }\end{array}$ & $\begin{array}{l}\text { Oxygen, } \\
\text { dissolved } \\
\text { (percent } \\
\text { satur- } \\
\text { ation) }\end{array}$ \\
\hline \multirow[t]{4}{*}{60} & $07-29-88$ & 0535 & 1.8 & 615 & 8.2 & 25.5 & 740 & 6.7 & 85 \\
\hline & $11-15-88$ & 1240 & 1.5 & 831 & 8.3 & 14.5 & 717 & 8.4 & 88 \\
\hline & 03-09-89 & 1230 & 2.5 & 748 & 8.2 & 6.5 & 751 & 14.2 & 117 \\
\hline & 06-01-89 & 1310 & .98 & 815 & 7.9 & 21.0 & 742 & 4.7 & 54 \\
\hline \multirow[t]{5}{*}{61} & $07-28-88$ & 1745 & 1,910 & 621 & 8.8 & 29.0 & 741 & 10.2 & 137 \\
\hline & $07-29-88$ & 0415 & 1,910 & 595 & 8.2 & 26.0 & 741 & 6.3 & 80 \\
\hline & $11-15-88$ & 1030 & 720 & 1,100 & 8.7 & 16.5 & 720 & 11.2 & 122 \\
\hline & 03-08-89 & 1030 & 909 & 1,040 & 8.3 & 1.0 & 748 & 13.6 & 98 \\
\hline & $05-31-89$ & 1710 & 759 & 892 & 8.4 & 27.0 & 734 & 7.9 & 103 \\
\hline \multirow[t]{6}{*}{62} & $07-28-88$ & 1815 & 2,280 & 633 & 8.3 & 30.0 & 744 & 9.7 & 132 \\
\hline & $07-29-88$ & 0530 & 2,250 & 642 & 7.5 & 26.0 & 742 & 6.0 & 76 \\
\hline & $07-29-88$ & 0535 & 2,250 & 642 & 7.5 & 26.0 & 742 & 6.0 & 76 \\
\hline & $11-15-88$ & 0755 & 809 & 983 & 8.5 & 15.0 & 723 & 11.6 & 122 \\
\hline & 03-07-89 & 1510 & 1,020 & 1,130 & 8.6 & 3.5 & 748 & 14.4 & 111 \\
\hline & $06-01-89$ & 1815 & 876 & 864 & 8.4 & 22.5 & 740 & 8.8 & 105 \\
\hline \multirow[t]{4}{*}{63} & $07-29-88$ & 0910 & .50 & 834 & 7.2 & 25.0 & 737 & 5.5 & 69 \\
\hline & $11-14-88$ & 1000 & .58 & 1,020 & 7.9 & 7.5 & 722 & 8.5 & 75 \\
\hline & 03-07-89 & 1010 & 1.2 & 730 & 8.1 & 3.0 & 743 & 13.6 & 104 \\
\hline & $06-01-89$ & 1300 & .36 & 698 & 7.6 & 19.5 & 732 & 6.7 & 76 \\
\hline \multirow[t]{10}{*}{64} & $07-28-88$ & 1230 & 6.4 & 504 & 8.3 & 30.0 & 743 & 8.8 & 120 \\
\hline & $07-28-88$ & 1235 & 6.4 & 504 & 8.3 & 30.0 & 743 & 8.8 & 120 \\
\hline & $07-28-88$ & 1830 & 6.4 & 490 & 8.3 & 30.0 & 742 & 7.5 & 102 \\
\hline & $07-29-88$ & 0005 & 6.4 & 524 & 7.9 & 26.0 & 740 & 6.3 & 80 \\
\hline & $07-29-88$ & 0435 & 6.4 & 522 & 7.9 & 25.0 & 742 & 5.4 & 67 \\
\hline & $11-14-88$ & 1530 & 3.9 & 645 & 8.5 & 17.0 & 734 & 17.2 & 185 \\
\hline & 03-07-89 & 1230 & 4.6 & 650 & 8.3 & 6.0 & 749 & 18.0 & 147 \\
\hline & $03-07-89$ & 1235 & 4.6 & 650 & 8.3 & 6.0 & 749 & 18.0 & 147 \\
\hline & 03-07-89 & 1330 & 4.6 & 655 & 8.3 & 7.0 & 749 & $>20.0$ & $>167$ \\
\hline & 03-07-89 & 1335 & 4.6 & 655 & 8.3 & 7.0 & 749 & $>20.0$ & $>167$ \\
\hline
\end{tabular}


Table 3. Results of water-quality determinations and dissolved-oxygen concentrations at synoptic-sampling sites in the lower Kansas River Basin, July 1988 through June 1989-Continued

\begin{tabular}{|c|c|c|c|c|c|c|c|c|c|}
\hline $\begin{array}{l}\text { Site map- } \\
\text { index } \\
\text { number } \\
\text { (fig. 10) }\end{array}$ & Date & $\begin{array}{c}\text { Time } \\
\text { (24-hour) }\end{array}$ & $\begin{array}{l}\text { Stream- } \\
\text { flow, } \\
\text { instan- } \\
\text { taneous } \\
\text { (cubic } \\
\text { feet per } \\
\text { second) }\end{array}$ & $\begin{array}{c}\text { Specific } \\
\text { conduct- } \\
\text { ance } \\
(\mu \mathrm{S} / \mathrm{cm})\end{array}$ & $\begin{array}{c}\text { pH } \\
\text { (standard } \\
\text { units) }\end{array}$ & $\begin{array}{c}\text { Temper- } \\
\text { ature, } \\
\text { water }\left({ }^{\circ} \mathrm{C}\right)\end{array}$ & $\begin{array}{c}\text { Baro- } \\
\text { metric } \\
\text { pressure } \\
\text { (mm of } \\
\mathrm{Hg})\end{array}$ & $\begin{array}{l}\text { Oxygen, } \\
\text { dissolved } \\
\text { (mg/L) }\end{array}$ & $\begin{array}{c}\text { Oxygen, } \\
\text { dissolved } \\
\text { (percent } \\
\text { satur- } \\
\text { ation) }\end{array}$ \\
\hline \multirow[t]{2}{*}{64} & $05-31-89$ & 1430 & 3.0 & 543 & 7.9 & 28.0 & 734 & 10.8 & 144 \\
\hline & $05-31-89$ & 1435 & 3.0 & 543 & 7.9 & 28.0 & 734 & 10.8 & 144 \\
\hline \multirow[t]{4}{*}{71} & $07-27-88$ & 0610 & 1.0 & 1,600 & 7.6 & 23.0 & 738 & 4.5 & 55 \\
\hline & $11-17-88$ & 1230 & 2.5 & 1,350 & 7.9 & 6.5 & 737 & 11.4 & 96 \\
\hline & $03-09-89$ & 1100 & 2.2 & 1,420 & 8.1 & 4.5 & 747 & 14.9 & 118 \\
\hline & $06-01-89$ & 1445 & 3.6 & -- & 7.7 & 20.0 & 737 & 7.0 & 79 \\
\hline \multirow[t]{13}{*}{72} & $07-27-88$ & 0745 & 2.4 & 677 & 7.8 & 20.5 & 739 & 6.2 & 71 \\
\hline & $08-31-88$ & 1025 & 1.5 & 694 & 8.3 & 20.5 & -- & 10.0 & -- \\
\hline & $10-05-88$ & 1000 & 1.5 & 636 & 8.7 & 10.5 & -- & 9.7 & -- \\
\hline & $11-09-88$ & 1020 & 2.5 & 860 & 8.3 & 8.5 & -- & 8.7 & -- \\
\hline & $11-17-88$ & 1020 & 22 & 816 & 8.4 & 5.5 & 743 & 12.1 & 99 \\
\hline & $12-21-88$ & 1010 & 9.6 & -- & 8.5 & 0 & -- & 10.5 & -- \\
\hline & $01-25-89$ & 1220 & 12 & 736 & 8.6 & 4.0 & -- & 11.2 & -- \\
\hline & $02-15-89$ & 1035 & E6.1 & 963 & 8.2 & 0 & -- & 13.5 & -- \\
\hline & 03-09-89 & 1230 & 14 & 602 & 8.4 & 9.0 & 749 & 15.1 & 133 \\
\hline & $03-15-89$ & 1135 & 9.9 & 569 & 8.8 & 9.0 & -- & 9.0 & -- \\
\hline & $04-19-89$ & 0925 & 4.7 & 717 & 8.4 & 10.0 & -- & 10.0 & -- \\
\hline & $05-17-89$ & 1320 & 2.5 & 917 & 8.3 & 19.5 & -- & 7.9 & -- \\
\hline & $06-02-89$ & 0945 & 2.1 & -- & 7.9 & 19.5 & 732 & 9.0 & -- \\
\hline \multirow[t]{11}{*}{73} & $07-27-88$ & 0810 & .60 & 697 & 7.7 & 20.5 & 738 & 5.2 & 60 \\
\hline & $11-17-88$ & 1300 & 3.5 & 730 & 8.1 & 6.5 & 740 & 12.6 & 106 \\
\hline & $11-17-88$ & 1305 & 3.5 & 730 & 8.1 & 6.5 & 740 & 12.6 & 106 \\
\hline & $11-17-88$ & 1345 & 3.5 & 730 & 8.1 & 6.5 & 740 & 12.6 & 106 \\
\hline & $11-17-88$ & 1350 & 3.5 & 730 & 8.1 & 6.5 & 740 & 12.6 & 106 \\
\hline & 03-09-89 & 1400 & 6.5 & 630 & 8.2 & 6.5 & 747 & 13.7 & 114 \\
\hline & 03-09-89 & 1405 & 6.5 & 630 & 8.2 & 6.5 & 747 & 13.7 & 114 \\
\hline & $03-09-89$ & 1440 & 6.5 & 630 & 8.2 & 6.5 & 747 & 13.7 & 114 \\
\hline & $03-09-89$ & 1445 & 6.5 & 630 & 8.2 & 6.5 & 747 & 13.7 & 114 \\
\hline & $06-02-89$ & 1100 & .79 & 753 & 7.9 & 22.0 & 735 & 8.3 & 99 \\
\hline & $06-02-89$ & 1105 & .79 & 753 & 7.9 & 22.0 & 735 & 8.3 & 99 \\
\hline
\end{tabular}


Table 3. Results of water-quality determinations and dissolved-oxygen concentrations at synoptic-sampling sites in the lower Kansas River Basin, July 1988 through June 1989-Continued

\begin{tabular}{|c|c|c|c|c|c|c|c|c|c|}
\hline $\begin{array}{l}\text { Site map- } \\
\text { index } \\
\text { number } \\
\text { (fig. 10) }\end{array}$ & Date & $\begin{array}{c}\text { Time } \\
\text { (24-hour) }\end{array}$ & $\begin{array}{l}\text { Stream- } \\
\text { flow, } \\
\text { instan- } \\
\text { taneous } \\
\text { (cubic } \\
\text { feet per } \\
\text { second) }\end{array}$ & $\begin{array}{c}\text { Specific } \\
\text { conduct- } \\
\text { ance } \\
(\mu \mathrm{S} / \mathrm{cm})\end{array}$ & $\begin{array}{c}\mathrm{pH} \\
\text { (standard } \\
\text { units) }\end{array}$ & $\begin{array}{c}\text { Temper- } \\
\text { ature, } \\
\text { water }\left({ }^{\circ} \mathrm{C}\right)\end{array}$ & $\begin{array}{c}\text { Baro- } \\
\text { metric } \\
\text { pressure } \\
\text { (mm of } \\
\text { Hg) }\end{array}$ & $\begin{array}{l}\text { Oxygen, } \\
\text { dissolved } \\
\text { (mg/L) }\end{array}$ & $\begin{array}{c}\text { Oxygen, } \\
\text { dissolved } \\
\text { (percent } \\
\text { satur- } \\
\text { ation) }\end{array}$ \\
\hline \multirow[t]{14}{*}{74} & $07-29-88$ & 1020 & 25 & 401 & 7.6 & 20.5 & 742 & 8.4 & 96 \\
\hline & $08-10-88$ & 1300 & 25 & 431 & 8.0 & 23.5 & -- & 7.9 & -- \\
\hline & $08-31-88$ & 1320 & 100 & 384 & 8.2 & 26.0 & -- & 8.2 & -- \\
\hline & $10-05-88$ & 1300 & 200 & 360 & 8.6 & 18.0 & -- & 9.6 & -- \\
\hline & $11-09-88$ & 1310 & 50 & 396 & 8.6 & 12.0 & - & 10.7 & -- \\
\hline & $11-15-88$ & 1430 & 50 & 378 & 8.4 & 11.5 & 724 & 9.0 & 87 \\
\hline & $12-21-88$ & 1315 & 25 & -- & 8.8 & 5.0 & -- & 13.2 & -- \\
\hline & $01-25-89$ & 0845 & 22 & 354 & 8.8 & 2.5 & -- & 11.3 & -- \\
\hline & $02-15-89$ & 1335 & 22 & 394 & 8.1 & 1.5 & -- & 10.7 & -- \\
\hline & 03-07-89 & 0800 & 22 & 389 & 7.8 & 2.5 & 758 & 12.9 & 95 \\
\hline & $03-15-89$ & 0845 & 22 & 390 & 8.3 & 8.0 & -- & 10.9 & -- \\
\hline & 04-19-89 & 1235 & 22 & 385 & 8.5 & 15.5 & -- & 13.1 & -- \\
\hline & $05-17-89$ & 1045 & 22 & 419 & 8.1 & 16.0 & -- & 8.9 & -- \\
\hline & $05-31-89$ & 1630 & 22 & 399 & 7.8 & 18.5 & 735 & 8.1 & 90 \\
\hline \multirow[t]{15}{*}{76} & $07-27-88$ & 1805 & 2,240 & 628 & 8.4 & 30.5 & 743 & 11.4 & 157 \\
\hline & $07-28-88$ & 0025 & 2,210 & 586 & 8.5 & 28.0 & 746 & 7.6 & 100 \\
\hline & $07-28-88$ & 0615 & 2,260 & 634 & 7.7 & 27.0 & 744 & 5.8 & 75 \\
\hline & $07-28-88$ & 1215 & 2,240 & 680 & 8.6 & 28.5 & 744 & 9.6 & 127 \\
\hline & $07-28-88$ & 1220 & 2,330 & 680 & 8.6 & 28.5 & 744 & 9.6 & 127 \\
\hline & $11-14-88$ & 0600 & 1,090 & 1,010 & 7.7 & 9.0 & 747 & 10.2 & 90 \\
\hline & $11-14-88$ & 0610 & 1,090 & 1,010 & 7.7 & 9.0 & 747 & 10.2 & 90 \\
\hline & $11-14-88$ & 1000 & 1,080 & 1,120 & 8.1 & 9.0 & 737 & 10.6 & 95 \\
\hline & $11-14-88$ & 1005 & 1,080 & 1,120 & 8.1 & 9.0 & 737 & 10.6 & 95 \\
\hline & $11-14-88$ & 1400 & 1,080 & 1,010 & 8.3 & 15.0 & 738 & 9.8 & 101 \\
\hline & $11-14-88$ & 1410 & 1,080 & 1,010 & 8.3 & 15.0 & 738 & 9.8 & 101 \\
\hline & $11-14-88$ & 1800 & 1,080 & 1,030 & 8.4 & 13.0 & 733 & 10.1 & 100 \\
\hline & $11-14-88$ & 1805 & 1,080 & 1,030 & 8.4 & 13.0 & 733 & 10.1 & 100 \\
\hline & $11-14-88$ & 2200 & 1,080 & 1,050 & 8.3 & 13.0 & 732 & 9.5 & 94 \\
\hline & $11-14-88$ & 2205 & 1,080 & 1,050 & 8.3 & 13.0 & 732 & 9.5 & 94 \\
\hline
\end{tabular}


Table 3. Results of water-quality determinations and dissolved-oxygen concentrations at synoptic-sampling sites in the lower Kansas River Basin, July 1988 through June 1989-Continued

\begin{tabular}{|c|c|c|c|c|c|c|c|c|c|}
\hline $\begin{array}{l}\text { Site map- } \\
\text { index } \\
\text { number } \\
\text { (fig. 10) }\end{array}$ & Date & $\begin{array}{c}\text { Time } \\
\text { (24-hour) }\end{array}$ & $\begin{array}{c}\text { Stream- } \\
\text { flow, } \\
\text { instan- } \\
\text { taneous } \\
\text { (cubic } \\
\text { feet per } \\
\text { second) }\end{array}$ & $\begin{array}{c}\text { Specific } \\
\text { conduct- } \\
\text { ance } \\
(\mu \mathrm{S} / \mathrm{cm})\end{array}$ & $\begin{array}{c}\text { pH } \\
\text { (standard } \\
\text { units) }\end{array}$ & $\begin{array}{l}\text { Temper- } \\
\text { ature, } \\
\text { water }\left({ }^{\circ} \mathrm{C}\right)\end{array}$ & $\begin{array}{c}\text { Baro- } \\
\text { metric } \\
\text { pressure } \\
\text { (mm of } \\
\text { Hg) }\end{array}$ & $\begin{array}{l}\text { Oxygen, } \\
\text { dissolved } \\
\text { (mg/L) }\end{array}$ & $\begin{array}{c}\text { Oxygen, } \\
\text { dissolved } \\
\text { (percent } \\
\text { satur- } \\
\text { ation) }\end{array}$ \\
\hline \multirow[t]{18}{*}{76} & $11-15-88$ & 0200 & 1,080 & 1,070 & 8.3 & 13.0 & 732 & 9.1 & 90 \\
\hline & $11-15-88$ & 0205 & 1,080 & 1,070 & 8.3 & 13.0 & 732 & 9.1 & 90 \\
\hline & $11-15-88$ & 0605 & 1,060 & 1,060 & 8.5 & 14.5 & 724 & 9.9 & 103 \\
\hline & 03-07-89 & 0615 & 1,010 & 1,180 & 8.2 & .5 & 758 & 14.1 & 99 \\
\hline & 03-07-89 & 1010 & 932 & 1,190 & 8.4 & .5 & 755 & 13.8 & 97 \\
\hline & 03-07-89 & 1015 & 932 & 1,190 & 8.4 & .5 & 755 & 13.8 & 97 \\
\hline & 03-07-89 & 1400 & 852 & 1,170 & 8.3 & 4.0 & 747 & 13.1 & 102 \\
\hline & 03-07-89 & 1405 & 852 & 1,170 & 8.3 & 4.0 & 747 & 13.1 & 102 \\
\hline & 03-07-89 & 1410 & 852 & 1,170 & 8.3 & 4.0 & 747 & 13.1 & 102 \\
\hline & 03-07-89 & 1800 & 892 & 1,240 & 8.4 & 5.5 & 752 & 12.8 & 103 \\
\hline & $03-07-89$ & 1805 & 892 & 1,240 & 8.4 & 5.5 & 752 & 12.8 & 103 \\
\hline & 03-07-89 & 2200 & 964 & 1,190 & 8.5 & 4.0 & 754 & 12.7 & 98 \\
\hline & 03-07-89 & 2205 & 964 & 1,190 & 8.5 & 4.0 & 754 & 12.7 & 98 \\
\hline & 03-08-89 & 0200 & 1,020 & 1,180 & 8.7 & 2.0 & 757 & 12.8 & 94 \\
\hline & $03-08-89$ & 0205 & 1,020 & 1,180 & 8.7 & 2.0 & 757 & 12.8 & 94 \\
\hline & 03-08-89 & 0600 & 1,070 & 1,310 & 8.7 & .5 & 748 & 13.0 & 92 \\
\hline & 03-08-89 & 0605 & 1,070 & 1,310 & 8.7 & .5 & 748 & 13.0 & 92 \\
\hline & $05-31-89$ & 1845 & 852 & 866 & 8.2 & 26.0 & 730 & 7.2 & 93 \\
\hline \multirow[t]{5}{*}{77} & $07-27-88$ & 1825 & 2,940 & 625 & 7.9 & 29.0 & 745 & 10.7 & 143 \\
\hline & $07-28-88$ & 0605 & $\mathrm{E} 2,940$ & 567 & 8.3 & 28.5 & 745 & 8.5 & 112 \\
\hline & $11-14-88$ & 1030 & 1,520 & 1,100 & 8.5 & 10.0 & 743 & 10.8 & 98 \\
\hline & $03-07-89$ & 1345 & 784 & 1,180 & 8.5 & 3.0 & 749 & 16.2 & 123 \\
\hline & $06-03-89$ & 0935 & 950 & 913 & 8.5 & 25.0 & 740 & 8.0 & 100 \\
\hline \multirow[t]{4}{*}{78} & $07-28-88$ & 0515 & 2,580 & 570 & 8.3 & 26.0 & 740 & 6.6 & 84 \\
\hline & $11-14-88$ & 1440 & 980 & 966 & 8.6 & 12.0 & 733 & 11.4 & 110 \\
\hline & $03-07-89$ & 1030 & 767 & 1,170 & 8.3 & 1.0 & 751 & 15.8 & 113 \\
\hline & $06-01-89$ & 1800 & 860 & 810 & 8.4 & 23.5 & 730 & 9.0 & 111 \\
\hline \multirow[t]{3}{*}{79} & $07-29-88$ & 0645 & .36 & 409 & 7.6 & 24.5 & 740 & 5.0 & 62 \\
\hline & 03-07-89 & 1600 & .40 & 528 & 8.0 & 6.5 & 748 & 12.0 & 100 \\
\hline & $05-31-89$ & 0930 & E. 15 & 507 & 7.6 & 24.0 & 736 & 4.5 & 56 \\
\hline
\end{tabular}


Table 3. Results of water-quality determinations and dissolved-oxygen concentrations at synoptic-sampling sites in the lower Kansas River Basin, July 1988 through June 1989-Continued

\begin{tabular}{|c|c|c|c|c|c|c|c|c|c|}
\hline $\begin{array}{l}\text { Site map- } \\
\text { index } \\
\text { number } \\
\text { (fig. 10) }\end{array}$ & Date & $\begin{array}{c}\text { Time } \\
\text { (24-hour) }\end{array}$ & $\begin{array}{l}\text { Stream- } \\
\text { flow, } \\
\text { instan- } \\
\text { taneous } \\
\text { (cubic } \\
\text { feet per } \\
\text { second) }\end{array}$ & $\begin{array}{c}\text { Specific } \\
\text { conduct- } \\
\text { ance } \\
(\mu \mathrm{S} / \mathrm{cm})\end{array}$ & $\begin{array}{c}\text { pH } \\
\text { (standard } \\
\text { units) }\end{array}$ & $\begin{array}{c}\text { Temper- } \\
\text { ature, } \\
\text { water }\left({ }^{\circ} \mathrm{C}\right)\end{array}$ & $\begin{array}{c}\text { Baro- } \\
\text { metric } \\
\text { pressure } \\
(\mathrm{mm} \text { of } \\
\mathbf{H g})\end{array}$ & $\begin{array}{l}\text { Oxygen, } \\
\text { dissolved } \\
\text { (mg/L) }\end{array}$ & $\begin{array}{c}\text { Oxygen, } \\
\text { dissolved } \\
\text { (percent } \\
\text { satur- } \\
\text { ation) }\end{array}$ \\
\hline \multirow[t]{14}{*}{83} & $07-29-88$ & 0530 & 24 & 320 & 7.8 & 26.0 & 741 & 6.7 & 85 \\
\hline & $08-10-88$ & 1500 & 23 & 339 & 8.3 & 29.0 & -- & 8.5 & -- \\
\hline & $08-31-88$ & 1500 & 240 & 313 & 8.4 & 25.5 & -- & 8.0 & -- \\
\hline & $10-05-88$ & 1500 & 28 & 291 & 8.3 & 16.5 & -- & 9.4 & -- \\
\hline & $11-09-88$ & 1555 & 9.2 & -- & 8.1 & 10.0 & -- & 8.3 & -- \\
\hline & $11-16-88$ & 1520 & 11 & 476 & 7.4 & 10.5 & -- & 2.7 & - \\
\hline & $12-21-88$ & 1515 & 4.9 & 375 & 8.3 & 4.0 & -- & 11.8 & -- \\
\hline & $01-25-89$ & 1605 & 8.6 & 300 & 7.9 & 6.0 & -- & 9.6 & -- \\
\hline & $02-15-89$ & 1535 & E6.2 & 386 & 8.2 & 1.0 & -- & 12.7 & - \\
\hline & $03-07-89$ & 1300 & 4.2 & 373 & 8.3 & 2.5 & 746 & 14.3 & 107 \\
\hline & $03-15-89$ & 1435 & 4.6 & 376 & 8.3 & 10.5 & -- & 10.2 & -- \\
\hline & $04-19-89$ & 1450 & 24 & 347 & 8.3 & 15.5 & -- & 9.6 & -- \\
\hline & $05-17-89$ & 0755 & 24 & 358 & 8.0 & 19.0 & -- & 7.1 & - \\
\hline & $05-31-89$ & 1045 & 22 & 340 & 7.8 & 24.5 & 738 & 5.9 & 73 \\
\hline \multirow[t]{5}{*}{86} & $07-28-88$ & 0615 & E.05 & 529 & 7.5 & 22.5 & 743 & 2.7 & 32 \\
\hline & $07-28-88$ & 0620 & E.05 & 529 & 7.5 & 22.5 & 743 & 2.7 & 32 \\
\hline & $07-28-88$ & 1200 & E. 40 & 515 & 7.8 & 25.5 & 745 & 7.0 & 88 \\
\hline & 03-08-89 & 1200 & 4.5 & 534 & 8.2 & 3.5 & 757 & 15.5 & 118 \\
\hline & $06-02-89$ & 1250 & E.30 & 557 & 7.8 & 23.5 & 736 & 8.1 & 99 \\
\hline \multirow[t]{6}{*}{87} & $07-28-88$ & 0445 & .06 & 481 & 7.7 & 25.5 & 745 & 5.4 & 68 \\
\hline & $07-28-88$ & 1500 & .06 & 474 & 8.1 & 29.0 & 745 & 8.5 & 113 \\
\hline & $11-14-88$ & 1345 & 2.4 & 490 & 7.8 & 10.0 & 735 & 8.4 & 77 \\
\hline & $11-14-88$ & 1350 & 2.4 & 490 & 7.8 & 10.0 & 735 & 8.4 & 77 \\
\hline & 03-08-89 & 1415 & 6.0 & 494 & 8.3 & 5.5 & 748 & 13.7 & 111 \\
\hline & $06-02-89$ & 1530 & 9.1 & 304 & 7.7 & 23.0 & 738 & 5.6 & 68 \\
\hline \multirow[t]{5}{*}{88} & $07-27-88$ & 1815 & 2,500 & 525 & 8.2 & 30.0 & 730 & 7.0 & 97 \\
\hline & $07-27-88$ & 2400 & 2,320 & 628 & 7.7 & 29.0 & 746 & 6.8 & 91 \\
\hline & $07-28-88$ & 0610 & 2,290 & 656 & 8.4 & 27.0 & 740 & 5.8 & 75 \\
\hline & $07-28-88$ & 1220 & 2,280 & 709 & 8.2 & 28.0 & 740 & 8.2 & 108 \\
\hline & $07-28-88$ & 1225 & 2,280 & 709 & 8.2 & 28.0 & 740 & 8.2 & 108 \\
\hline
\end{tabular}


Table 3. Results of water-quality determinations and dissolved-oxygen concentrations at synoptic-sampling sites in the lower Kansas River Basin, July 1988 through June 1989-Continued

\begin{tabular}{|c|c|c|c|c|c|c|c|c|c|}
\hline $\begin{array}{l}\text { Site map- } \\
\text { index } \\
\text { number } \\
\text { (fig. 10) }\end{array}$ & Date & $\begin{array}{c}\text { Time } \\
\text { (24-hour) }\end{array}$ & $\begin{array}{l}\text { Stream- } \\
\text { flow, } \\
\text { instan- } \\
\text { taneous } \\
\text { (cubic } \\
\text { feet per } \\
\text { second) }\end{array}$ & $\begin{array}{c}\text { Specific } \\
\text { conduct- } \\
\text { ance } \\
(\mu \mathrm{S} / \mathrm{cm})\end{array}$ & $\begin{array}{c}\text { pH } \\
\text { (standard } \\
\text { units) }\end{array}$ & $\begin{array}{l}\text { Temper- } \\
\text { ature, } \\
\text { water }\left({ }^{\circ} \mathrm{C}\right)\end{array}$ & $\begin{array}{c}\text { Baro- } \\
\text { metric } \\
\text { pressure } \\
(\mathrm{mm} \text { of } \\
\mathrm{Hg})\end{array}$ & $\begin{array}{l}\text { Oxygen, } \\
\text { dissolved } \\
\text { (mg/L) }\end{array}$ & $\begin{array}{r}\text { Oxygen, } \\
\text { dissolved } \\
\text { (percent } \\
\text { satur- } \\
\text { ation) }\end{array}$ \\
\hline \multirow[t]{8}{*}{88} & $11-16-88$ & 1510 & 1,060 & 1,080 & 8.5 & 9.5 & 739 & 10.0 & 91 \\
\hline & $11-16-88$ & 1515 & 1,060 & 1,080 & 8.5 & 9.5 & 739 & 10.0 & 91 \\
\hline & 03-07-89 & 1550 & 608 & 1,380 & 8.8 & 8.0 & 750 & 11.6 & 100 \\
\hline & $03-07-89$ & 1555 & 608 & 1,380 & 8.8 & 8.0 & 750 & 11.6 & 100 \\
\hline & 03-07-89 & 1630 & 608 & 1,380 & 8.8 & 8.0 & 750 & 11.6 & 100 \\
\hline & 03-07-89 & 1635 & 608 & 1,380 & 8.8 & 8.0 & 750 & 11.6 & 100 \\
\hline & $05-30-89$ & 1905 & 1,180 & 927 & 8.6 & 28.0 & 735 & 10.4 & 138 \\
\hline & $05-30-89$ & 1910 & 1,180 & 927 & 8.6 & 28.0 & 735 & 10.4 & 138 \\
\hline \multirow[t]{8}{*}{90} & $07-29-88$ & 0510 & 9.9 & 972 & 7.7 & 24.0 & 740 & 2.9 & 36 \\
\hline & $07-29-88$ & 0515 & 9.9 & 972 & 7.7 & 24.0 & 740 & 2.9 & 36 \\
\hline & $11-14-88$ & 1210 & 24 & 1,030 & 8.1 & 16.0 & 736 & 7.4 & 78 \\
\hline & $11-14-88$ & 1215 & 24 & 1,030 & 8.1 & 16.0 & 736 & 7.4 & 78 \\
\hline & $11-14-88$ & 1315 & 24 & 1,000 & 7.8 & 17.0 & 736 & 6.4 & 69 \\
\hline & $11-14-88$ & 1320 & 24 & 1,000 & 7.8 & 17.0 & 736 & 6.4 & 69 \\
\hline & 03-07-89 & 0925 & 28 & 1,510 & 8.4 & 6.0 & 752 & 12.0 & 98 \\
\hline & $06-02-89$ & 1440 & 32 & 1,100 & 7.9 & 23.0 & 739 & 7.3 & 88 \\
\hline \multirow[t]{4}{*}{91} & $07-29-88$ & 0615 & $\mathrm{E} 2,550$ & 723 & 8.4 & 28.0 & 740 & 5.5 & 73 \\
\hline & $11-14-88$ & 1000 & 1,010 & 923 & 8.3 & 11.5 & 736 & 8.4 & 80 \\
\hline & 03-07-89 & 1320 & 1,090 & -- & 9.0 & 6.0 & 752 & 11.8 & -- \\
\hline & $06-02-89$ & 1145 & 1,180 & 1,170 & 8.0 & 26.0 & 744 & 5.0 & 63 \\
\hline
\end{tabular}


selected sites and times and are denoted by a time 5 minutes later than the primary determination. Duplicate analyses reported in table 8 were performed on splits of single water samples.

\section{Areal Variations}

Areal variations in predawn DO concentrations for the July 1988 synoptic survey are shown in figure 11 . Of the 31 sites shown in figure 11, 5 had concentrations smaller than the $5.0-\mathrm{mg} / \mathrm{L}, 1$-day minimum, warmwater criterion for early life stages. Concentrations at 4 of these 5 sites were less than the $3.0-\mathrm{mg} / \mathrm{L}$ criterion for all other life stages. These 5 sites were: Lincoln Creek near Aurora, Nebr. (site 9), West Fork Big Blue River near Hastings, Nebr. (site 16), Delaware River near Fairview, Kans. (site 71), Stranger Creek at Easton, Kans. (site 86), and Turkey Creek at Kansas City, Kans. (site 90). DO concentrations for these sites are listed in table 3.

The DO deficiencies at sites 9, 16, and 90 appear to be the result of discharge of oxygen-demanding material from a municipal wastewater-treatment facility in excess of the assimilation capacity of the stream. Site 9 is approximately 1.25 stream mi downstream from the Aurora, Nebr., municipal-wastewater discharge point. The USEPA permitted discharge for this facility is $0.37 \mathrm{Mgal} / \mathrm{d}$ or an average discharge of $0.57 \mathrm{ft}^{3} / \mathrm{s}$. Measured streamflow at site 9 on July 26, 1988 , was $0.34 \mathrm{ft}^{3} / \mathrm{s}$ (table 3); therefore, a substantial part of this streamflow probably originated as wastewater discharge. Measurement of DO on subsequent synoptic surveys showed similar concentrations to those observed on July 26 , with the exception of the November 15,1988 , measurement of $4.4 \mathrm{mg} / \mathrm{L}$. Although a DO concentration of $4.4 \mathrm{mg} / \mathrm{L}$ appears large in relation to other DO data and nutrient concentrations, it may be the result of larger streamflow $\left(1.4 \mathrm{ft}^{3} / \mathrm{s}\right)$ that probably produced greater surface turbulence, greater reaeration, and cooler water temperature $\left(6.5^{\circ} \mathrm{C}\right)$, which contributed to greater oxygen solubility. However, $4.4 \mathrm{mg} / \mathrm{L}$ was only 39 percent of saturation, similar to the July 26,1988 (2150 hours), observation.

Site 16 is about 2 mi downstream from the Hastings, Nebr., wastewater-treatment facility. The permitted discharge for this facility is $2.75 \mathrm{Mgal} / \mathrm{d}$ $\left(4.26 \mathrm{ft}^{3} / \mathrm{s}\right)$ and, thus, conceivably accounts for a substantial part of the $6.7 \mathrm{ft}^{3} / \mathrm{s}$ streamflow measured at site 16 on July 26, 1988 (table 3 ). The $2.1-\mathrm{mg} / \mathrm{L}$, predawn (0630 hours) DO concentration measured on July 26,1988 , was considerably less than the 3.0-mg/L, 1-day minimum criteria for adult organisms. Additionally, a DO measurement was made at 1800 hours on July 26 to estimate maximum diel variability and the result of photosynthesis. This second measurement $(4.8 \mathrm{mg} / \mathrm{L})$ was less than the $5.0-\mathrm{mg} / \mathrm{L}$ criterion. In fact, no DO measurements at site 16 for any of the synoptic surveys were greater than the $5.0-\mathrm{mg} / \mathrm{L}$ criterion, and furthermore, the smallest DO concentration observed in water at any site during any synoptic survey was at site 16 on November 15,1988 $(0.6 \mathrm{mg} / \mathrm{L})$. The exact length of the West Fork Big Blue River adversely affected by wastewater discharge is not known; however, the predawn DO concentration at the next downstream sampling site (site 17) was $5.9 \mathrm{mg} / \mathrm{L}$ at 0500 hours on July 27,1988 , and nutrient concentrations were considerably smaller. Site 17 is 43 stream mi from site 16.

Site 90 is approximately $2 \mathrm{mi}$ downstream from a major wastewater-treatment facility, which has a permitted discharge rate of $15 \mathrm{Mgal} / \mathrm{d}$. The effect of this discharge during the July 1988 synoptic survey is evident at site 90 . The predawn ( 0510 hours) DO concentration was $2.9 \mathrm{mg} / \mathrm{L}$ on July 29,1988 . Concentrations of nutrients and organic carbon were exceptionally large at this site and were some of the largest observed at any site during any synoptic survey. However, DO concentrations less than the 5.0- $\mathrm{mg} / \mathrm{L}$ criteria were not observed at site 90 during any of the other surveys. This can be attributed to a combination of larger flow rates, cooler water temperatures, and the fact that predawn sampling (when DO concentrations would be minimal) was not conducted at this site during subsequent synoptic surveys.

The predawn DO concentration at site 71 was $4.5 \mathrm{mg} / \mathrm{L}$ on July 27,1988 . The flow at this site includes discharge from the Sabetha, Kans., wastewater-treatment facility, which is located about 12 mi upstream from site 71 , but the effect of this discharge on DO concentrations at site 71 appears to be minimal. An examination of nutrient concentrations from this site indicates no unusually large concentrations that may indicate wastewater discharges; therefore, it appears that the reach of stream upstream of site 71 is adequate for the assimilation of the $0.13 \mathrm{Mgal} / \mathrm{d}$ permitted discharge from this facility. The small DO concentration observed on July 27 is pro- 


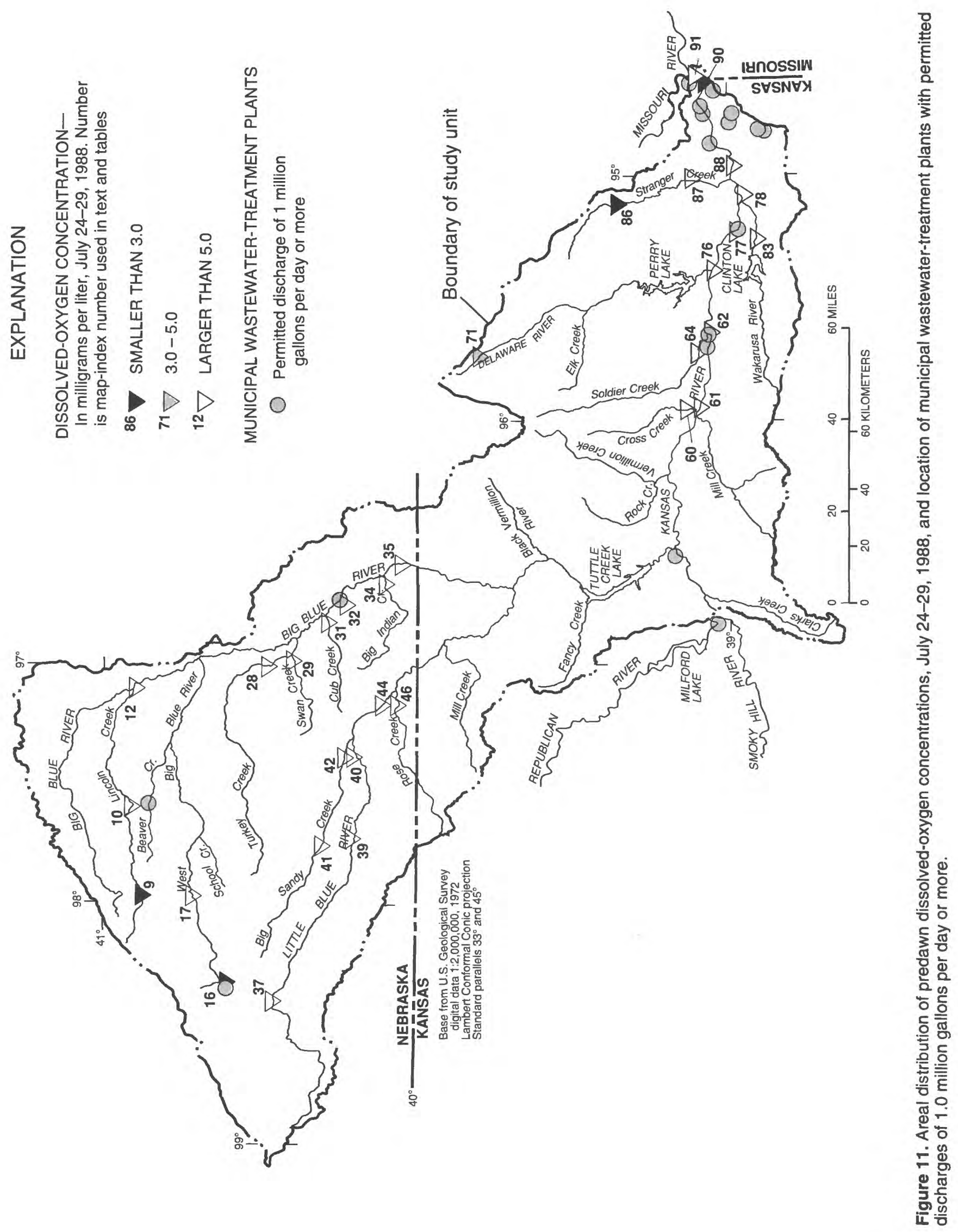


bably the result of reduced reaeration during low flow and of algal respiration. Although the algal biomass, as indicated by a chlorophyll- $a$ concentration of $15 \mu \mathrm{g} / \mathrm{L}$ (micrograms per liter) (table 8), was not exceptionally large compared to other sites, it probably was sufficient to create an oxygen depression during the night. In addition to the free-floating algae, large accumulations of periphyton have been observed at this site. Periphyton respiration also would place an oxygen demand on the stream. No other DO concentrations less than the criteria were observed at this site during subsequent surveys.

The predawn DO concentration at site 86 was $2.7 \mathrm{mg} / \mathrm{L}$ on July 28,1988 . Upstream of site 86 there are two wastewater-treatment facilities with a combined permitted discharge of $0.07 \mathrm{Mgal} / \mathrm{d}$. However, these facilities are about $20 \mathrm{mi}$ upstream of site 86 and are believed to have little effect at this site. The small predawn DO concentration observed on July 28 probably was the result of inadequate physical reaeration associated with extremely small streamflow $\left(0.05 \mathrm{ft}^{3} / \mathrm{s}\right)$ and of algal respiration. When the site was revisited at 1200 hours on July 28 , streamflow had increased to $0.40 \mathrm{ft}^{3} / \mathrm{s}$, and the DO concentration to $7.0 \mathrm{mg} / \mathrm{L}$. The unusual increase in streamflow might have been the result of beaver activity upstream from site 86. Beavers have built dams on the upstream reaches of Stranger Creek, including site 86, for many years. Their nocturnal construction activities can substantially reduce streamflow for short periods of time. The increase in streamflow observed on July 28 may be attributed to a breach in one of these upstream dams or may represent a reestablishment of normal base flow following dam construction. Another possible explanation may be the withdrawal of upstream water for irrigation purposes, ceasing sometime after 0615 hours, and subsequent resumption of normal flow. The $7.0-\mathrm{mg} / \mathrm{L} \mathrm{DO}$ concentration observed at 1200 hours probably is the result of reaeration and photosynthesis. No other DO concentrations measured at site 86 during subsequent surveys were less than established criteria.

DO concentrations were measured as part of the ancillary data collected with synoptic surveys for nutrients in November 1988 and March and May-June 1989. Four of those measurements were less than established criteria: $4.9 \mathrm{mg} / \mathrm{L}$, Beaver Creek near York, Nebr. (site 21), May 31, 1989; 4.7 mg/L, Cross Creek at Rossville, Kans. (site 60), June 1, 1989; 4.5 $\mathrm{mg} / \mathrm{L}$, Wakarusa River near Berryton, Kans. (site 79),
May 31, 1989; and $2.7 \mathrm{mg} / \mathrm{L}$, Wakarusa River near Lawrence, Kans. (site 83), November 16, 1988.

Site 21 is located approximately $5 \mathrm{mi}$ downstream from the 1.28-Mgal/d (permitted) municipal wastewater-treatment facility at York, Nebr. Although discharge from this facility did not seem to have an adverse effect on DO concentrations during the July 1988 synoptic survey, subsequent surveys identified large concentrations of nutrients, particularly nitrite plus nitrate-nitrogen and phosphorus. Discharge from the facility may have caused the 4.9-mg/L DO concentration measured on May 31, 1989.

The DO concentrations measured at sites 60 and 79 during the May-June 1989 synoptic survey were slightly less than the $5.0-\mathrm{mg} / \mathrm{L}$ criterion. This probably resulted from a combination of reduced physical reaeration associated with low flow $\left(0.98 \mathrm{ft}^{3} / \mathrm{s}\right.$ at site 60 and $0.15 \mathrm{ft}^{3} / \mathrm{s}$ at site 79) and decomposition of organic material accelerated by warmer water. Upstream from site 60 are two municipal wastewatertreatment facilities with a combined permitteddischarge rate of $0.8 \mathrm{Mgal} / \mathrm{d}$; however, the nearest of these facilities is more than 10 stream mi from site 60 . An examination of nutrient concentrations for site 60 shows small concentrations, which seems to indicate that the effects of wastewater discharge are not evident at this sampling site. No wastewater-treatment facilities are upstream from site 79; however, a beaver dam was located immediately upstream of the sampling site. Decomposition of bottom material in the placid water behind the dam, a reduction in physical reaeration associated with extremely low streamflow, and the relatively early-morning (0930 hours) observation, all may contribute to the $4.5-\mathrm{mg} / \mathrm{L}$ DO concentration measured on May 31, 1989.

The 2.7-mg/L DO concentration measured at site 83 on November 16,1988, probably was the result of oxygen-demanding material transported into the stream by an isolated, short-duration rain the day of sampling. Observer notes for November 16 indicated that the stream was turbid with a very fine suspended material resulting from localized runoff from adjacent farmland. Site 83 is downstream of Clinton Lake and does not receive discharge from wastewater-treatment facilities.

Concentrations of DO in violation of water-quality criteria in the lower Kansas River Basin are localized occurrences and do not reflect regional variation in DO. Generally, the most severe DO deficiencies are the result of discharges from municipal wastewater- 
treatment facilities into small tributary streams with inadequate dilution or assimilation capacity. Algal respiration in combination with reduced physical reaeration associated with low streamflow also may cause temporary, localized deficiencies in DO concentrations. Oxygen demand from the decomposition of benthic material in pooled or placid streams may serve to compound the effect of reduced physical reaeration.

Concentrations of DO less than water-quality criteria were not observed on major tributaries or the main stem of the Kansas River. DO concentrations appear to be relatively similar throughout the basin. When mean predawn concentrations are compared between sites on the Big Blue River (sites 12, 32, 35), Little Blue River (sites 37, 39, 40, 44), and Kansas River (sites $61,62,76,77,78,88,91$ ), there is little appreciable difference $(6.23 \mathrm{mg} / \mathrm{L}, 6.60 \mathrm{mg} / \mathrm{L}$, and $6.36 \mathrm{mg} / \mathrm{L}$, respectively). Of the 31 sites where predawn DO measurements were made, 5 sites (16 percent) had concentrations less than water-quality criteria. Of all DO concentrations measured (392) in conjunction with synoptic surveys (table 3 ), only 9 measurements ( 2.3 percent) were less than waterquality criteria.

\section{Diel Variability}

Daily DO fluctuations and diel variability are largely dependent on temperature fluctuations and photosynthesis-respiration relations. In slow, shallow streams (typical of the lower Kansas River Basin) that contain an abundance of phytoplankton, periphyton, and rooted aquatic plants, daytime photosynthetic production exceeds physical reaeration as a source of oxygen and exceeds consumption from respiration. The result frequently is supersaturation during the day, followed by a decrease to less than saturation at night. Depending upon oxygen demand, daily minimum DO concentrations usually occur prior to early-morning water-temperature lows (Reid and Wood, 1976, p. 215). DO concentrations may be acutely affected by sunlight intensity. The considerable diel variability in DO indicates the importance of more than a single DO measurement to adequately define the oxygen regime in a stream.

To describe DO diel variability in the lower Kansas River Basin, 11 sites were sampled during July 1988 , at 4- or 6-hour intervals for approximately
24 hours. Results of DO measurements for these sites are shown in figure 12. Results of ancillary onsite measurements are listed in table 3. Results of chemical analysis of water samples for nutrients, organic carbon, chlorophyll, and suspended sediment collected during these diel studies are listed in table 8 in the "Supplemental Information" section of this report. At site 32, Big Blue River at Beatrice, Nebr., the DO measurement at 0500 hours on July 26,1988 , was inadvertently omitted during the survey. The DO concentration for 0500 hours shown in figure 12 for site 32 was assumed to be equal to the $5.8-\mathrm{mg} / \mathrm{L}$ concentration measured at 0510 hours on July 27.

At nearly every site, minimum DO concentrations occurred just before dawn (about 0600 hours), and maximum concentrations occurred in late afternoonearly evening (about 1800 hours). Concentrations of DO less than the $5.0-\mathrm{mg} / \mathrm{L}$ criterion were not measured at any of the diel-study sites. The largest DO concentrations measured were at site 44 , Little Blue River near Fairbury, Nebr. (10.6 mg/L) and site 76, Kansas River at Lecompton, Kans. (11.4 mg/L). The largest ranges in DO concentration also occurred at these two sites ( 5.3 and $5.6 \mathrm{mg} / \mathrm{L}$, respectively). Two of the three largest chlorophyll concentrations (chlorophyll- $a$ plus chlorophyll- $b$ ) occurred at site 44 and site 76 (113 and $85 \mu \mathrm{g} / \mathrm{L}$, respectively, table 8 ).

As a result of diel variability, DO shown in figure 12 and table 3 was supersaturated during the day and less than saturation during the night except at sites 32 and 46. Maximum DO at all sites ranged from 94-percent saturation at site 46 to 157 percent at site 76, with a mean maximum of 115 percent. Minimum DO at all sites ranged from 66-percent saturation at site 12 to 79 percent at site 34 , with a mean minimum of 72 percent. Mean DO using all measurements at all sites was 92-percent saturation. It appears that diel variability in streams not affected by localized problems is basically a function of the photosynthesisrespiration relation and, to a lesser extent, a function of the temperature-atmospheric, pressure-solubility relation.

Seasonal effects on diel variability were examined at site 32, Big Blue River at Beatrice, Nebr., and site 76, Kansas River at Lecompton, Kans. Diel studies were conducted at both sites in July and November 1988 and March 1989; and at site 32 in May 1989. Results of these diel studies are shown in figure 13. The effect of water temperature on oxygen solubility is evident in figure 13. Water temperatures at both sites 

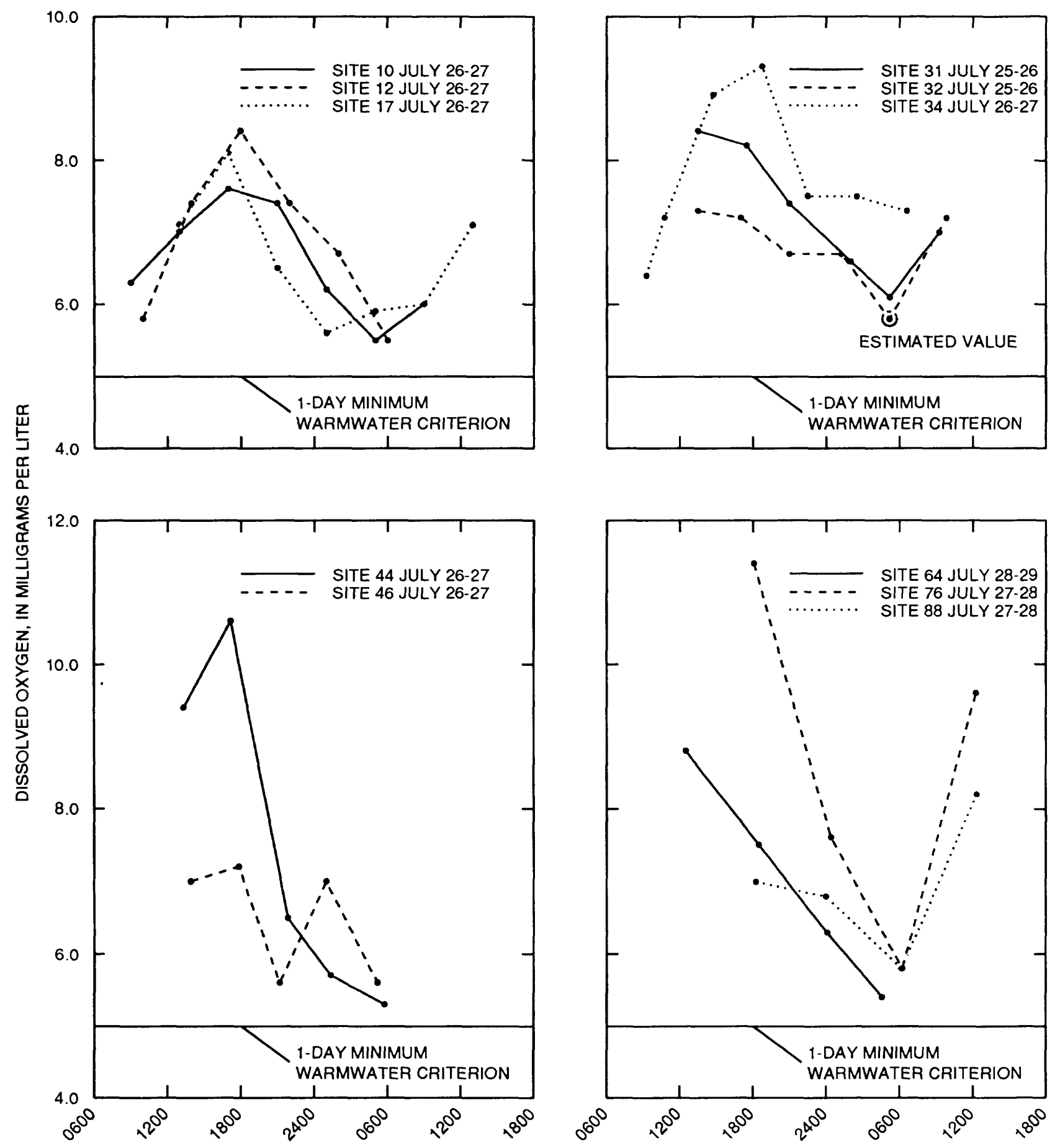

TIME (24-HOUR)

Figure 12. Diel variability of dissolved-oxygen concentrations at selected synoptic-sampling sites in the lower Kansas River Basin for survey of July 1988 (criterion established by U.S. Environmental Protection Agency, 1986). 


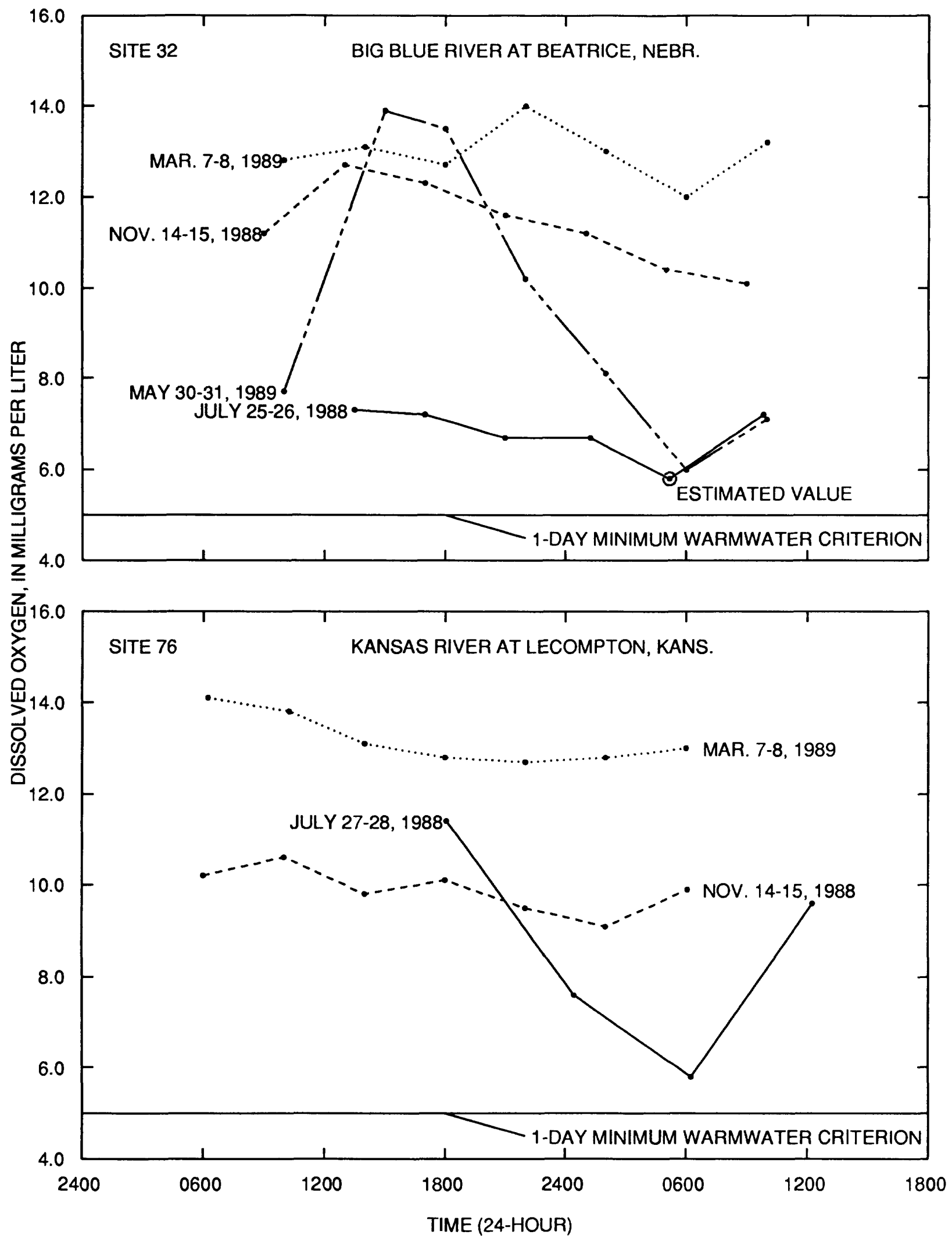

Figure 13. Seasonal effect on the diel variability of dissolved-oxygen concentrations in the Big Blue River at Beatrice, Nebr., and the Kansas River at Lecompton, Kans. (criterion established by U.S. Environmental Protection Agency, 1986). 
ranged from 25.0 to $30.5^{\circ} \mathrm{C}$ in July $1988,7.0$ to $15.0^{\circ} \mathrm{C}$ in November 1988,0 to $5.5^{\circ} \mathrm{C}$ in March 1989 , and 21.5 to $25.0^{\circ} \mathrm{C}$ in May 1989. As water temperature decreased, DO concentrations increased, and generally, diel variability decreased. The decreased diel variability was most pronounced at site 76 .

During the July 1988 survey at site 76 , the diel variability of DO concentrations was $5.6 \mathrm{mg} / \mathrm{L}$. Variabilities for the November 1988 and March 1989 surveys were 1.5 and $1.4 \mathrm{mg} / \mathrm{L}$, respectively. This reduced diel variability can be attributed to smaller algal biomass and cooler water, which reduces the rate of biochemical reactions including photosynthesis and respiration. Reduction in algal biomass is shown by chlorophyll concentrations of March 7, 1989, which averaged $5.9 \mu \mathrm{g} / \mathrm{L}$ for three replicate samples (table 8 ). This contrasts with the $85-\mu \mathrm{g} / \mathrm{L}$ concentration on July 28,1988 , when the water was considerably warmer.

Generally, DO concentrations during the coldweather months are a function of the water temperature. This can be seen in the gradual reduction in DO concentrations during the daylight hours as the water warms. Maximum DO concentrations occur before noon when water temperature is coolest, the reverse of the warm-weather response. This was most evident during the November 1988 survey at sites 32 and 76 and the March 1989 survey at site 76.

\section{ESCHERICHIA COLI DENSITIES}

\section{Sources and Water-Quality Criteria}

The bacterium Escherichia coli $(E$. coli) is indigenous solely to the intestinal tract of warmblooded animals. It is a member of the family Enterobacteriaceae and the predominate bacterium in the fecal coliform group (McKinney, 1962).

Therefore, the presence of $E$. coli in the aquatic environment is an indication of fecal contamination. This contamination may come from municipal wastewater discharges; leachate from domestic septic systems, runoff or ground-water seepage from livestockproducing areas (pasture and confined feedlots), or wildlife populations.

Seyfried and others (1984) state that municipal wastewater discharges can have a detrimental effect on the water quality of receiving streams not only in regard to fecal bacteria but also to the introduction of pathogenic organisms, such as reo-, adeno-, and enterovirus; coxsackievirus; and poliovirus. The studies of Stephenson and Street (1978), Doran and others (1981), and Gary and others (1983) have demonstrated the effect of cattle production on fecal coliform densities in runoff from grazed and pastured land and in streams adjacent to these areas. Kress and Gifford (1984) attempted to quantify release of fecal coliforms from cattle fecal deposits of various ages and under varying rainfall intensities. Hagedorn and others (1978) have shown that $E$. coli can survive lateral movement through soil at sites with as little as 2-percent slope.

The survival characteristics of $E$. coli in aquatic environments of various chemical compositions were studied by Lim and Flint (1989). They found that in filtered, autoclaved lake water, $E$. coli survived for 12 days without a decline in viable counts. The addition of synthetic sewage to unfiltered water led to an increase in viable counts and also to an increase in survival time relative to unfiltered water without synthetic sewage. The addition of a nitrogen source, likewise, increased survival times and was proportional to the concentration of the nitrogen source.

For many years prior to 1986 , densities of fecal coliform and total coliform bacteria were traditional measures of sanitary quality. In 1986, the USEPA revised the ambient water-quality criteria for marine and freshwater (U.S. Environmental Protection Agency, 1986) and recommended that criteria for E. coli and the Enterococci organisms replace criteria for fecal coliform and total coliform bacteria in State water-quality regulations for the protection of primary-contact recreation. The USEPA has established several levels of human-health protection with regard to the single-sample maximum allowable density of $E$. coli based on frequency of use of the water body. The least stringent of these levels, infrequently used full-body contact recreation, has been set at $576 \mathrm{col} / 100 \mathrm{~mL}$ (colonies per 100 milliliters of water). Additionally, the Kansas Department of Health and Environment has established a criterion for fecal coliform of 2,000 col/100 mL for those streams available for all beneficial uses other than primary (fullbody) contact recreation (Fromm and Wilk, 1988). Because the fecal coliform group may contain bacteria other than $E$. coli, the $2,000-\mathrm{col} / 100 \mathrm{~mL}$ criterion, if applied to $E$. coli, may result in a less-stringent criterion than that currently established (that is, $2,000 \mathrm{E}$. -coli col/100 $\mathrm{mL}$ probably equates to more 
than 2,000 fecal coliform col/100 mL). However, because few streams or segments of streams in the study unit are classified by the Kansas Department of Health and Environment or the Nebraska Department of Environmental Control for full-body contact, it is appropriate that some criterion for other than primary contact be used for evaluating $E$. coli densities observed during this study. Therefore, both the $2,000-\mathrm{col} / 100 \mathrm{~mL}$ criterion and the $576-\mathrm{col} / 100 \mathrm{~mL}$ criterion will be used in this report. These criteria are applicable only during stable-flow, dry-weather conditions.

\section{Data Collection and Analysis}

Water samples for determination of $E$. coli densities were collected July 24-29, 1988, in conjunction with a synoptic survey for determination of DO concentrations. A followup synoptic survey of $E$. coli was conducted July 16-21, 1989, to verify results obtained the previous year. The location of synopticsampling sites are described in table 2 and shown in figure 10.

Samples at 57 sites were collected during stable, low-streamflow, conditions. Samples representative of the stream cross section were collected using the equal-width-increment (EWI) method and a sterile DH-81 depth-integrating suspended-sediment sampler on wadeable streams or a sterile D-77 suspendedsediment sampler where depth necessitated collection from a bridge (Guy and Norman, 1970). The EWI method has been described previously in the "Study Approach" section of this report; however, samples for E. coli analysis were not composited in a churn splitter. On small, shallow streams where depthintegrating samplers were inappropriate, a sample was collected by hand dipping a sterilized samplecollection bottle into the centroid of flow. Sampler nozzles, gaskets, and collection bottles were cleaned and autoclaved for 20 minutes at $121{ }^{\circ} \mathrm{C}$ and $15 \mathrm{lb} / \mathrm{in}^{2}$ prior to use. These parts were replaced between sampling sites to prevent cross contamination of samples.

Samples were chilled after collection and transported to a centrally located site for analysis. Samples collected in the upper Big Blue River and Little Blue River subbasins were analyzed in Beatrice, Nebr. Samples collected along the main stem Kansas River and tributaries were analyzed at the U.S. Geological Survey laboratory in Lawrence, Kans.
The membrane filter (MF) method was used for the detection and enumeration of $E$. coli densities (U.S. Environmental Protection Agency, 1985). The MF method provides a direct count of bacteria based on the development of colonies on the membrane filter after a water sample has been filtered through it. Subsequently, the membrane containing the bacteria cells is placed on a selective and differential medium, mTEC, incubated at $35^{\circ} \mathrm{C}$ for 2 hours to resuscitate injured or stressed bacteria, and then incubated at $44.5^{\circ} \mathrm{C}$ for 22 hours to promote colony growth. Following incubation, the filter is transferred to a filter pad saturated with urea substrate. After 15 minutes, $E$. coli will become yellow or yellow brown and are counted under a lamp and magnifying lens.

Samples were filtered and the incubation process started as soon as possible after collection. Samples were chilled until processed, but no sample was held longer than 6 hours. Sterile, disposable, polystyrene filter units with 0.45 -micrometer-porosity membrane filters were used to filter samples. One filter unit was used per water sample. Different membrane filters were used for each filtered sample volume. Sample volumes ranged from 0.1 to $100 \mathrm{~mL}$ (milliliters) in a series of five or six volumes. Sample volumes were filtered in ascending order. Plate counts were converted to densities in colonies per $100 \mathrm{~mL}$ of sample. Because of the potential for slight variations in analytical procedure between analysts, a single analyst processed, filtered, and counted all samples collected for both synoptic surveys. This was done to limit fluctuation in analytical precision and maintain comparability among sites. Results for determination of $E$. coli densities for synoptic surveys of July 1988 and July 1989 are listed in table 4.

\section{Associated Rainfall and Streamflow Characteristics}

The objectives of conducting a synoptic survey for $E$. coli densities were to define the spatial occurrence of fecal contamination, document the effect of major point-source discharges, and describe the relation between fecal contamination and factors such as land use, agricultural practices, and waste-management practices. For determinations of $E$. coli densities to be comparable between sampling sites, it was necessary to sample all sites under similar hydrologic conditions. Sampling to meet these objectives is most appro- 


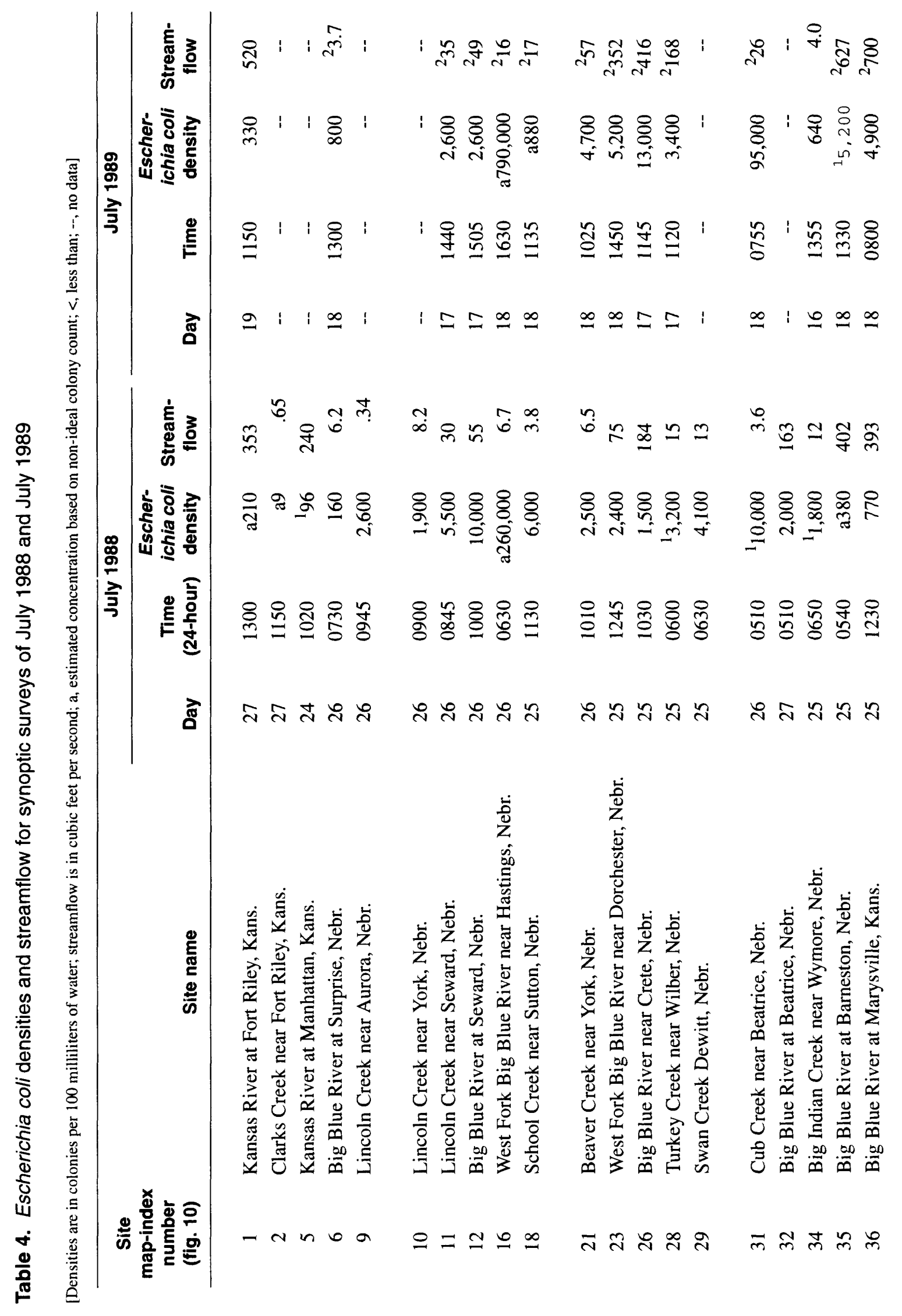




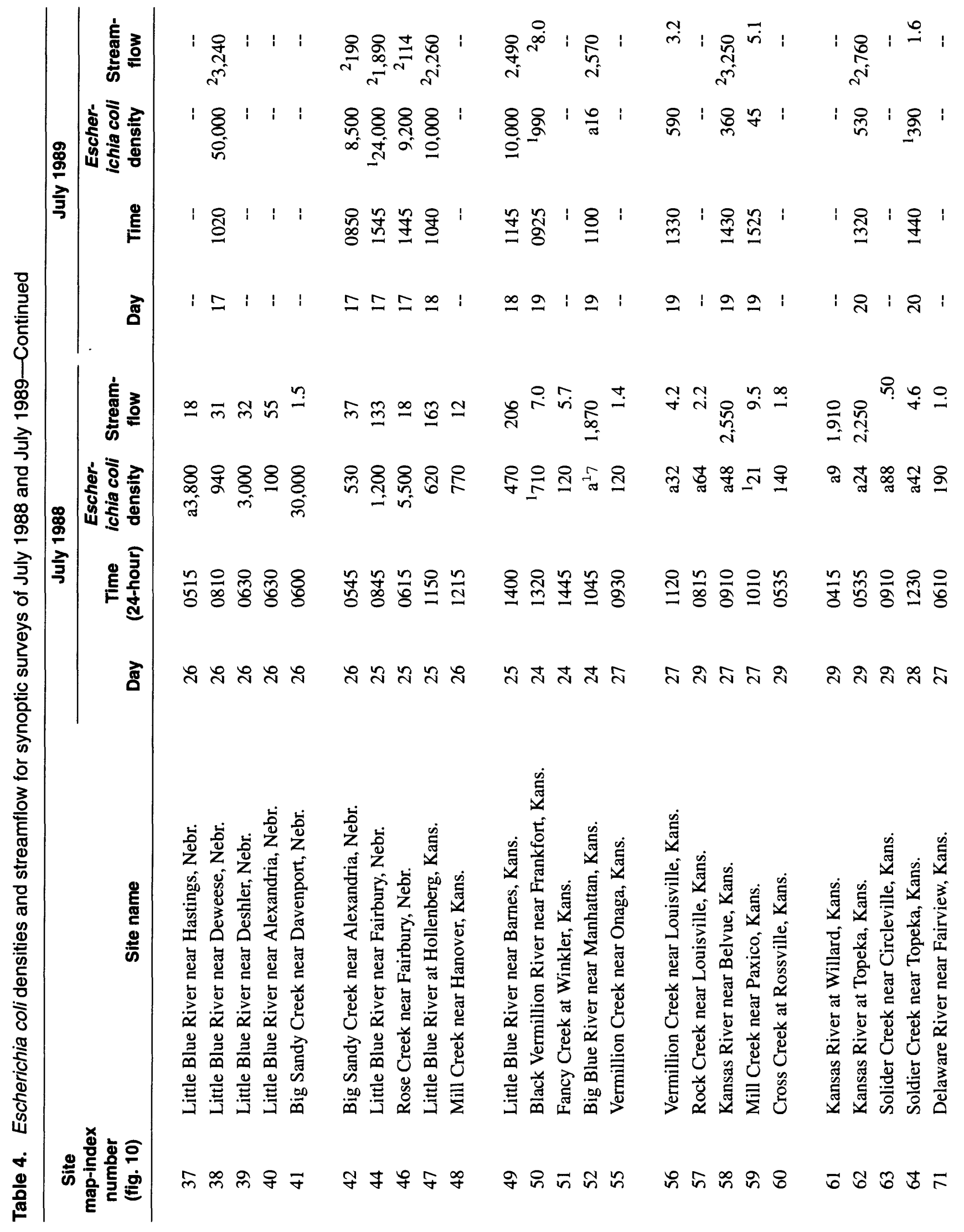




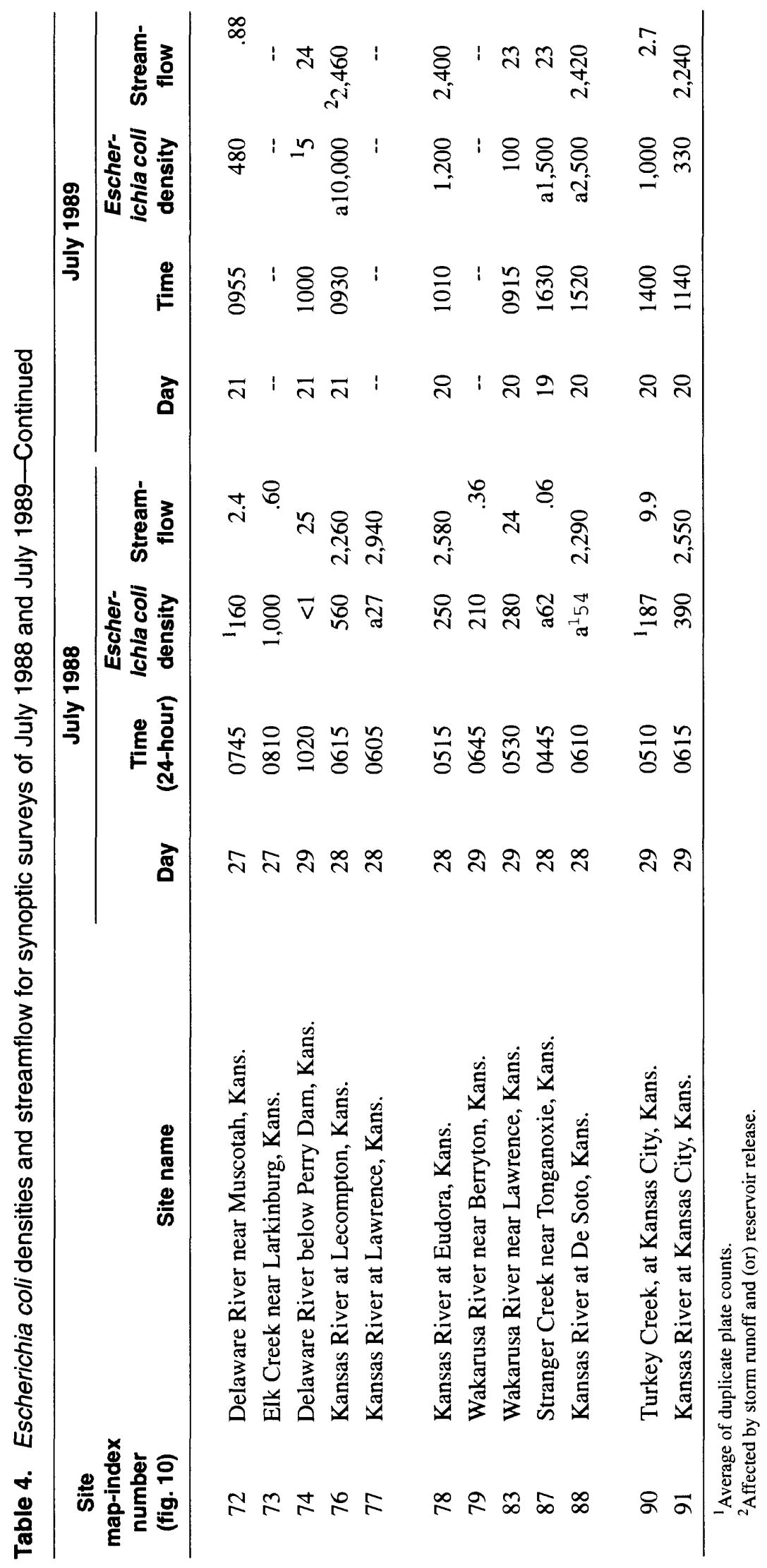


priately conducted during stable low-flow conditions when effects of overland runoff would be less likely to cause large fluctuations in $E$. coli densities. Therefore, an evaluation of rainfall before and during sampling periods was needed to define the potential for or occurrences of overland runoff. An evaluation of streamflow was necessary to determine whether baseflow conditions prevailed.

Antecedent and concurrent rainfall characteristics for the July 1988 and July 1989 synoptic surveys are listed in table 5. No rainfall was recorded at any of the six locations listed in table 5 for at least 4 days before the start of the July 1988 survey. Total rainfall during the 2 weeks before the July 1988 survey ranged from 0.53 in. at Topeka, Kans., to 3.53 in. at Manhattan, Kans. During the 6 days of the survey, rainfall ranged from zero at Hastings, Nebr., and Manhattan, Kans., to 0.22 in. at Marysville, Kans. Based on these rainfall characteristics plus an examination of hydrographic records and field observations, it was concluded that none of the sites sampled during the July 1988 synoptic survey were affected by nonpoint-source contributions from overland runoff as a result of rainfall. However, irrigation-water return flow was observed at one site in the upper Big Blue River subbasin. The effect of irrigation-water return flow on streamflow at base-flow conditions, both from a hydrologic and water-quality aspect, may be substantial in the upper Big Blue River and Little Blue River subbasins. As previously indicated in figure 4 , the amount of irrigated acreage in a representative area of these subbasins has increased substantially since the 1930's. With this increase in irrigation has come an increase in return flow to area streams probably affecting both streamflow and water quality. Figure 6 shows that the majority of irrigation wells in the study unit are located in the upper Big Blue River and Little Blue River subbasins.

Rainfall characteristics before and during the July 1989 synoptic survey were considerably wetter than those of the July 1988 survey. No dry days immediately preceded the start of the July 1989 survey at any of the six locations listed in table 5 (some rainfall was recorded at every location on July 15, 1989). Rainfall recorded during the 3 days prior to July 16, 1989, ranged from 0.02 in. at Manhattan, Kans., to 2.50 in. at Topeka, Kans. However, the largest rainfall amounts recorded during the survey were at those locations in the Big Blue River subbasin and ranged from 0.58 in. at Beatrice, Nebr., to 1.06 in. at Hastings,
Nebr. Because of the wet antecedent conditions and substantial rainfall during the survey, streamflows at most sites in the Big Blue River and Little Blue River subbasins were affected by overland runoff.

Occurrence of base-flow conditions during synoptic surveys in July 1988 and July 1989 can be evaluated by an examination of flow-duration data for the observed streamflows. Table 6 lists selected sampling sites, date and time of sampling, streamflow, and percentage of time streamflow is equalled or exceeded. Based on long-term records, the mean percentage of time that streamflow during the July 1988 synoptic survey is equalled or exceeded at main-stem sites on the Big Blue River (sites 6, 12, 32, 36), Little Blue River (sites 38, 47), and Kansas River (sites 1, 58, 62, 76,88 ) were 42 percent, 81 percent, and 70 percent, respectively. Streamflow on the upper Big Blue River during the July 1988 survey was not at extreme low flow; however, it was at stable conditions based on hydrographic record and observations made at the time of sampling. The relatively large flows at the Big Blue River sites were probably the result of groundwater discharge resulting from rainfall during the previous 2 weeks and, possibly as important, from irrigation-water return flow. Streamflow in the Kansas River downstream of Manhattan was stable but somewhat increased because of a reservoir release from Tuttle Creek Lake. The Big Blue River near Manhattan, Kans. (site 52), downstream of Tuttle Creek Lake, was at a flow rate equalled or exceeded only 29 percent of the time. The release from Tuttle Creek Lake kept the Kansas River downstream of Manhattan, Kans., at a flow rate equalled or exceeded an average of 63 percent (sites 58,62,76,88) of the time compared to the upstream most Kansas River site at Fort Riley, Kans. (site 1), which had a flow rate equalled or exceeded 95 percent of the time.

Generally, streamflows in the Big Blue River and Little Blue River subbasins for the July 1989 synoptic survey were either larger than streamflows during the July 1988 survey or were affected by overland runoff from recent rains. The Big Blue River at Surprise, Nebr. (site 6), and near Seward, Nebr. (site 12), had flow rates similar to those sampled in July 1988 but were affected by overland runoff as determined by an examination of hydrographic records and field observations. Streamflows in the Big Blue River subbasin downstream of Seward, Nebr., and in the Little Blue River subbasin were affected substantially by overland runoff (sites 23, 28, 36, 38, 42, 47). The largest 
Table 5. Rainfall characteristics at selected locations for synoptic surveys of July 1988 and July 1989

[Data from National Oceanic and Atmospheric Administration, 1988-89a, b; rainfall values are in inches]

\begin{tabular}{|c|c|c|c|c|c|c|c|c|}
\hline \multirow[b]{3}{*}{ Location (fig. 1) } & \multicolumn{4}{|c|}{ July 24-29, 1994} & \multicolumn{4}{|c|}{ July 16-21, 1989} \\
\hline & \multirow{2}{*}{$\begin{array}{l}\text { Number } \\
\text { of } \\
\text { antece- } \\
\text { dent dry } \\
\text { days }\end{array}$} & \multicolumn{2}{|c|}{ Antecedent rainfall } & \multirow{2}{*}{$\begin{array}{c}\text { Total } \\
\text { rainfall } \\
\text { during } \\
\text { sampling } \\
\text { period }\end{array}$} & \multirow{2}{*}{$\begin{array}{c}\text { Number } \\
\text { of } \\
\text { antece- } \\
\text { dent dry } \\
\text { days }\end{array}$} & \multicolumn{2}{|c|}{ Antecedent rainfall } & \multirow{2}{*}{$\begin{array}{c}\text { Total } \\
\text { rainfall } \\
\text { during } \\
\text { sampling } \\
\text { period }\end{array}$} \\
\hline & & $\begin{array}{c}\text { Prior } 3 \\
\text { days }\end{array}$ & $\begin{array}{c}\text { Prior } 14 \\
\text { days }\end{array}$ & & & $\begin{array}{c}\text { Prior } 3 \\
\text { days }\end{array}$ & $\begin{array}{c}\text { Prior } 14 \\
\text { days }\end{array}$ & \\
\hline York, Nebr. & 4 & 0 & 1.65 & 0.09 & 0 & 0.88 & 0.92 & 0.63 \\
\hline Hastings, Nebr. & 4 & 0 & 1.57 & 0 & 0 & 1.98 & 2.21 & 1.06 \\
\hline Beatrice, Nebr. & 4 & 0 & 2.28 & .03 & 0 & 1.47 & 1.54 & .58 \\
\hline Marysville, Kans. & 4 & 0 & 2.23 & .22 & 0 & .80 & 2.79 & .25 \\
\hline Manhattan, Kans. & 4 & 0 & 3.53 & 0 & 0 & .02 & .58 & .38 \\
\hline Topeka, Kans. & 7 & 0 & .53 & .08 & 0 & 2.50 & 2.88 & .40 \\
\hline
\end{tabular}

streamflows were in the upper Little Blue River subbasin. The Little Blue River near Deweese, Nebr. (site 38), had a flow rate equalled or exceeded only 0.4 percent of the time. Streamflow along the main stem Kansas River and tributary streams during the July 1989 survey were similar to those observed during the July 1988 survey. However, because of fluctuations in releases from Tuttle Creek Lake, several Kansas River sites (sites 58, 62, 76) were not under stable-flow conditions. No sites on the main stem Kansas River or tributary streams appeared to be affected by recent overland runoff.

\section{Quality Assurance}

Within-site variations in $E$. coli counts may be the result of variability in (1) analytical procedures, (2) sample-collection procedures, (3) natural microbial distribution, and (4) differences in techniques of multiple analysts. Procedures were developed to evaluate or control these sources of variation to the extent possible. Other quality-assurance procedures included verification of the sterility of the buffered-dilution water and equipment used in the filtration process.

Variation in $E$. coli counts caused by analytical procedures was evaluated by filtering duplicate volumes of samples collected at 10 randomly selected sites during the July 1988 synoptic survey. Two to four duplicate volumes were filtered for each site, producing a total of 28 duplicate pairs. Counts of $E$. coli colonies were made for the duplicate pairs (table 7), and variation was evaluated with a technique used by Dufour and others (1981) to quantify the precision of the mTEC procedures. They calculated variation as a percentage of the mean with the equation:

$$
\text { variation }=\frac{\mathrm{s} / \sqrt{\mathrm{n}}}{\overline{\mathrm{x}}} \cdot 100
$$

where

$s$ is the standard deviation,

$n$ is the number of duplicates, and

$\bar{x}$ is the mean count of the duplicate pairs.

For a sample size of two, variation can be shown to be expressed as:

$$
\text { variation }=\frac{\left(\mathrm{x}_{1}-\mathrm{x}_{2}\right) / 2}{\overline{\mathrm{x}}} \cdot 100
$$

The variations computed by equation 2 for the duplicate-pair counts are listed in table 7 . Average variation for the mTEC analytical procedure as determined from all data in table 7 is 22 percent of the mean. Average variation for duplicate pairs with both counts within the ideal counting range of $20-80 \mathrm{col} / 100 \mathrm{~mL}$ is 10 percent of the mean. Average variation for duplicate pairs with one or both counts within the ideal counting range is 13 percent of the mean. Average variation for duplicate pairs where 


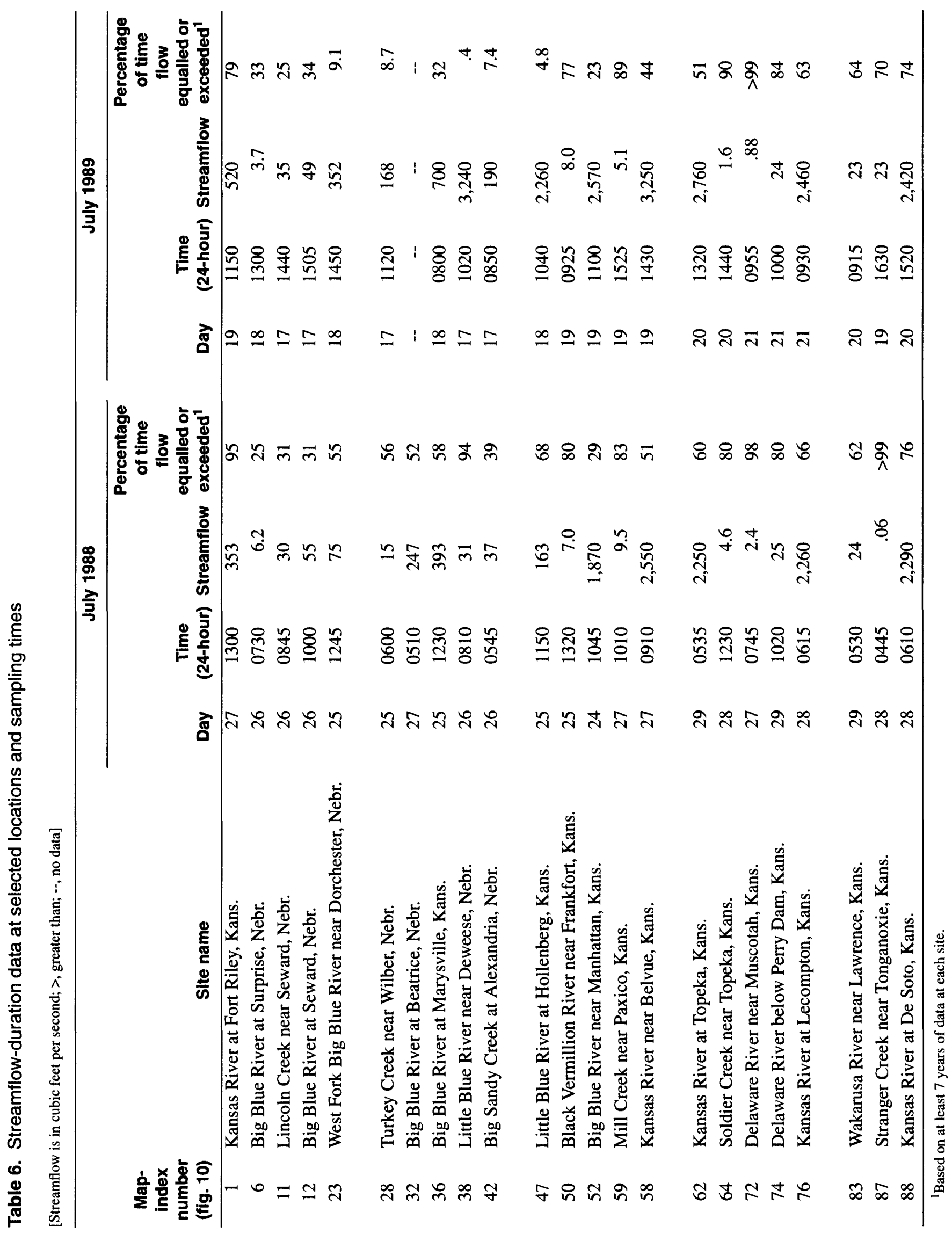


Table 7. Variation of Escherichia coli counts in two-plate duplicate-volume filtrations from samples collected during synoptic survey of July 1988

[Variation is computed by equation 2; Escherichia coli counts are actual plate counts]

\begin{tabular}{|c|c|c|c|}
\hline \multirow{2}{*}{$\begin{array}{c}\text { Site } \\
\text { map-index } \\
\text { number } \\
\text { (fig. 10) }\end{array}$} & \multicolumn{2}{|c|}{ Escherichia coli counts } & \multirow{2}{*}{$\begin{array}{c}\text { Variation, } \\
\text { in percent } \\
\text { of mean }\end{array}$} \\
\hline & $\begin{array}{c}\text { Plate } 1 \\
\left(x_{1}\right)\end{array}$ & $\begin{array}{c}\text { Plate } 2 \\
\left(x_{2}\right)\end{array}$ & \\
\hline \multirow[t]{3}{*}{5} & 2 & 1 & 33 \\
\hline & 8 & 10 & 11 \\
\hline & 19 & 29 & 21 \\
\hline \multirow[t]{3}{*}{28} & 1 & 5 & 67 \\
\hline & 24 & 17 & 17 \\
\hline & 54 & 50 & 4 \\
\hline \multirow[t]{3}{*}{31} & 8 & 11 & 16 \\
\hline & 41 & 41 & 0 \\
\hline & 115 & 95 & 10 \\
\hline \multirow[t]{3}{*}{34} & 6 & 9 & 20 \\
\hline & 30 & 26 & 7 \\
\hline & 83 & 55 & 20 \\
\hline \multirow[t]{3}{*}{50} & 1 & 3 & 50 \\
\hline & 14 & 8 & 27 \\
\hline & 53 & 38 & 16 \\
\hline \multirow[t]{2}{*}{52} & 3 & 2 & 20 \\
\hline & 7 & 6 & 8 \\
\hline \multirow[t]{2}{*}{59} & 2 & 1 & 33 \\
\hline & 20 & 22 & 5 \\
\hline \multirow[t]{3}{*}{72} & 7 & 4 & 27 \\
\hline & 13 & 12 & 4 \\
\hline & 49 & 32 & 21 \\
\hline \multirow[t]{4}{*}{87} & 3 & 1 & 50 \\
\hline & 6 & 8 & 14 \\
\hline & 10 & 12 & 9 \\
\hline & 44 & 31 & 17 \\
\hline \multirow[t]{2}{*}{88} & 4 & 11 & 47 \\
\hline & 6 & 11 & 29 \\
\hline
\end{tabular}

neither count was within the ideal counting range is 26 percent. Therefore, as counts deviate from the ideal counting range, variation increases considerably.

Variations in $E$. coli counts caused by samplecollection procedures (the ability to collect a representative sample of the bacterial population) and natural bacterial distribution were not examined in this study because micro-organisms are rarely distributed randomly and because bacteria within any habitat will have a clumped or patchy distribution (Britton and Greeson, 1987, p. 4) and will produce uncertainty in computed variation when viewed from a temporal perspective. Under these conditions, the ability to distinguish between variation caused by sample-collection procedures and that caused by distributional patterns may be questionable without devoting substantial effort to collect and process a sufficient number of samples to define the contribution of both potential sources of variability. Additionally, within-site variation may be site specific, and assumptions made based on computed variation at a particular site may not be true for other sites in the synoptic survey. A detailed examination of sample-collection variability and natural variability in distribution at individual synoptic sites was beyond the scope of this study.

Variations in $E$. coli counts caused by variability in analytical technique among multiple analysts were not of concern in this study. As previously described, a single analyst filtered, plated, and counted all $E$. coli samples. This same analyst performed these functions for both the July 1988 and July 1989 synoptic surveys.

Four equipment-blank samples using sterile, buffered dilution water were filtered and plated on mTEC medium. After incubation, none of the plates showed development of $E$. coli colonies. This lack of development verified the sterility of the buffered water used for sample dilutions and equipment used during the filtration process. Also, it indicated that the filtration equipment and procedure were not a source of contamination.

\section{Areal Variations}

The areal variations of $E$. coli densities for the July 1988 survey are shown in figure 14 . Densities of E. coli in water at the 19 sites in the Big Blue River subbasin, exclusive of the Little Blue River subbasin, upstream of Tuttle Creek Lake (sites 6-36, 50, and 51, table 7) ranged from $120 \mathrm{col} / 100 \mathrm{~mL}$ in Fancy Creek at Winkler, Kans. (site 51), to $260,000 \mathrm{col} / 100 \mathrm{~mL}$ in the West Fork Big Blue River near Hastings, Nebr. (site 16). Densities at the 11 sites in the Little Blue River subbasin (sites 37-49, table 7) ranged from $100 \mathrm{col} / 100 \mathrm{~mL}$ in the Little Blue River near Alexandria, Nebr. (site 40 ), to $30,000 \mathrm{col} / 100 \mathrm{~mL}$ at 


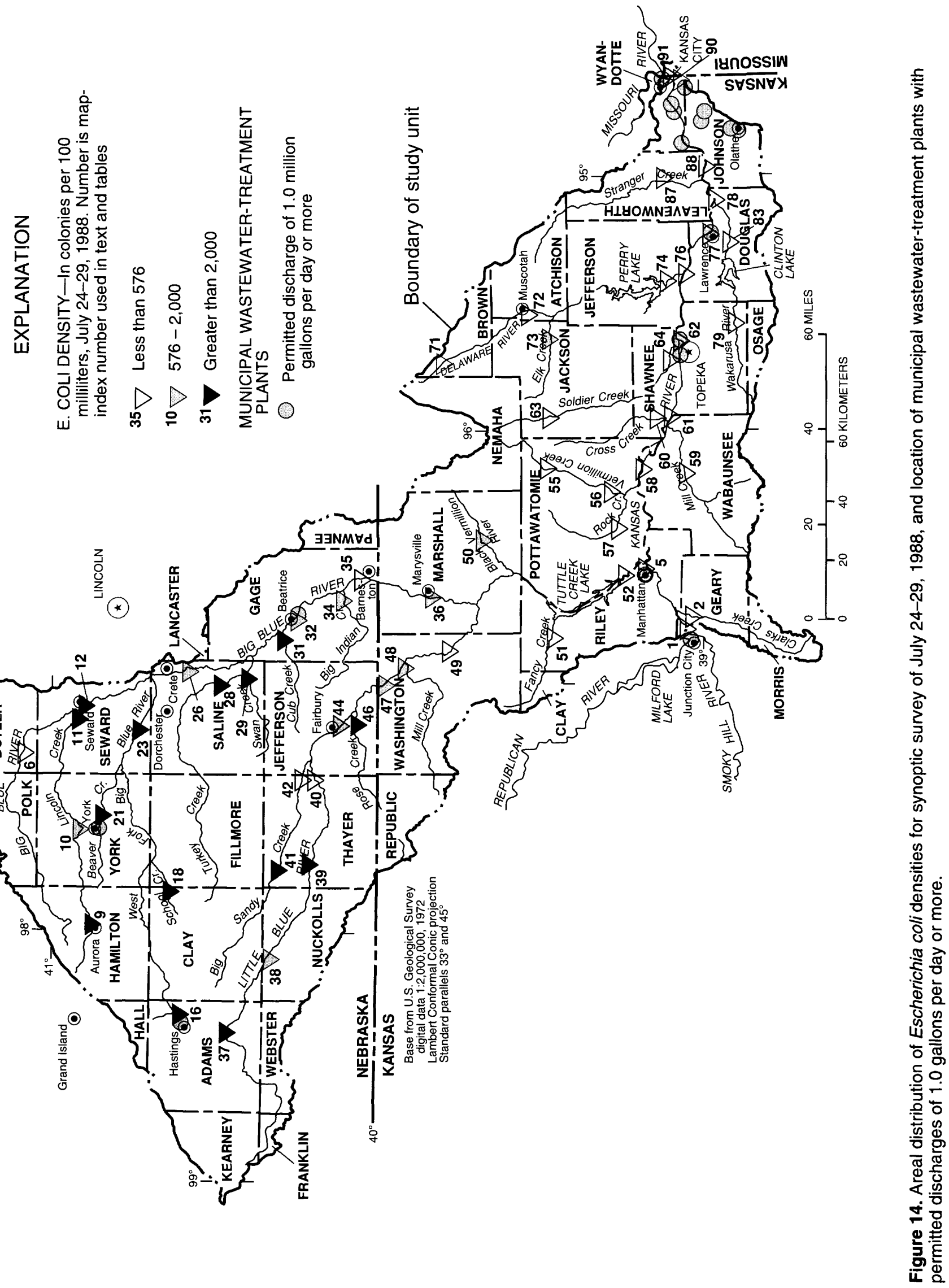


Big Sandy Creek near Davenport, Nebr. (site 41). Densities of $E$. coli at the 27 sites located in the Kansas River subbasin (sites 1, 2, 5, and 52-91, table 7) ranged from less than $1 \mathrm{col} / 100 \mathrm{~mL}$ in the Delaware River below Perry Dam, Kans. (site 74), to $1,000 \mathrm{col} / 100 \mathrm{~mL}$ in Elk Creek near Larkinburg, Kans. (site 73).

Of the 19 sites sampled in the Big Blue River subbasin upstream of Tuttle Creek Lake, stream water at 16 of those sites ( 84 percent) exceeded the USEPA criterion for $E$. coli densities $(576 \mathrm{col} / 100 \mathrm{~mL}$ ) for infrequently used full-body contact recreation, and 10 sites (53 percent) exceeded the $2,000-\mathrm{col} / 100 \mathrm{~mL}$ fecal coliform criterion for uses other than full-body contact established by the Kansas Department of Health and Environment (Fromm and Wilk, 1988). A downstream-ordered distribution of $E$. coli densities for the Big Blue River subbasin is shown in figure 15 . For tributary streams only the downstream-most sampling site is shown. Generally, downstream from the Big Blue River at Seward, Nebr. (site 12), densities of $E$. coli decreased on both the main stem and tributary streams probably because of a combination of dilution and bacterial die off.

The $E$. coli density $(10,000 \mathrm{col} / 100 \mathrm{~mL})$ at site 12 apparently reflects not only the contribution $(5,500 \mathrm{col} / 100 \mathrm{~mL})$ from Lincoln Creek (site 11) but also a significant nonpoint-source contribution. Site 12 is upstream of the Seward municipal wastewatertreatment plant and, thus, is not affected by its discharge. Upstream of site 12 , there are only two small wastewater-treatment plants--the municipal wastewater-treatment plant at Staplehurst, 12 stream mi upstream, with a permitted discharge of $0.01 \mathrm{Mgal} / \mathrm{d}$ and Ulysses, 28 stream mi upstream, with a permitted discharge of $0.04 \mathrm{Mgal} / \mathrm{d}$. Because of the distances upstream of these two wastewater-treatment plants and their small permitted discharge rates, it is believed that these two point sources would have little effect on $E$. coli densities at site 12 . Therefore, it appears that a large part of the $E$. coli density determined at site 12 was from nonpoint-source contributions. These contributions probably originated as irrigation return flow or ground-water discharge and were related to livestock production upstream of site 12. As previously indicated in table 1 and figures 7 and 8 , production of cattle and hogs in this area are at some of the highest levels in the study unit.

The relatively large $E$. coli density $(10,000 \mathrm{col} / 100 \mathrm{~mL}$ ) in Cub Creek (site 31) was probably of nonpoint-source origin. The only known point-source discharge of $E$. coli is the Jensen municipal wastewater-treatment plant, which has a permitted discharge rate of $0.02 \mathrm{Mgal} /$ day. This plant is located on a tributary to Cub Creek many miles upstream of site 31 . Therefore, its effect on $E$. coli densities at site 31 is believed to be minimal. As is common in much of the northern part of the study unit, livestock production in the Cub Creek Basin is substantial (table 1, figs. 7 and 8) and probably contributes to the occurrence of $E$. coli in local streams.

Of the 11 sites sampled for $E$. coli in the Little Blue River subbasin, 8 (73 percent) exceeded the $576 \mathrm{col} / 100 \mathrm{~mL}$ primary-contact criterion, and 4 (36 percent) exceeded the $2,000-\mathrm{col} / 100 \mathrm{~mL}$ criterion for other than primary contact. Figure 16 shows a downstream-ordered distribution of $E$. coli densities in the Little Blue River subbasin as determined during the July 1988 synoptic survey. The density of $E$. coli in the Little Blue River near Hastings (site 37$)$ was the largest $(3,800 \mathrm{col} / 100 \mathrm{~mL})$ determined at any main-stem site and may have come in large part from nonpoint sources. Upstream from site 37 are three small wastewater-treatment plants; however, their location ( 4.6 to 41 stream mi upstream) and combined total permitted discharge $(0.08 \mathrm{Mgal} / \mathrm{d})$ are believed to have minimal effect on $E$. coli density at site 37 . The much smaller density $(940 \mathrm{col} / 100 \mathrm{~mL})$ determined at Little Blue River near Deweese, Nebr. (site 38), may indicate that the 26-mi stream reach between sites 37 and 38 is an area of $E$. coli die off, with no exceptionally large nonpoint-source contribution. There are no known point sources between sites 37 and 38 .

The increase in E. coli density $(3,000 \mathrm{col} / 100 \mathrm{~mL})$ in the Little Blue River near Deshler, Nebr. (site 39), may have been the result of discharge from the Deshler wastewater-treatment plant 3 stream mi upstream from site 39. Permitted discharge for the wastewater-treatment plant is $0.10 \mathrm{Mgal} / \mathrm{d}$. The $100-\mathrm{col} / 100 \mathrm{~mL}$ E. coli density determined at the Little Blue River near Alexandria, Nebr. (site 40), represents the smallest $E$. coli density determined in the Little Blue River subbasin, in spite of the fact that it is downstream of the Hebron, Nebr., wastewatertreatment plant, which has a permitted discharge of $0.16 \mathrm{Mgal} / \mathrm{d}$. However, the 18 stream mi between the Hebron wastewater-treatment plant and site 40 may provide enough distance for self purification, may give some insight into $E$. coli die-off rates, and may indi- 


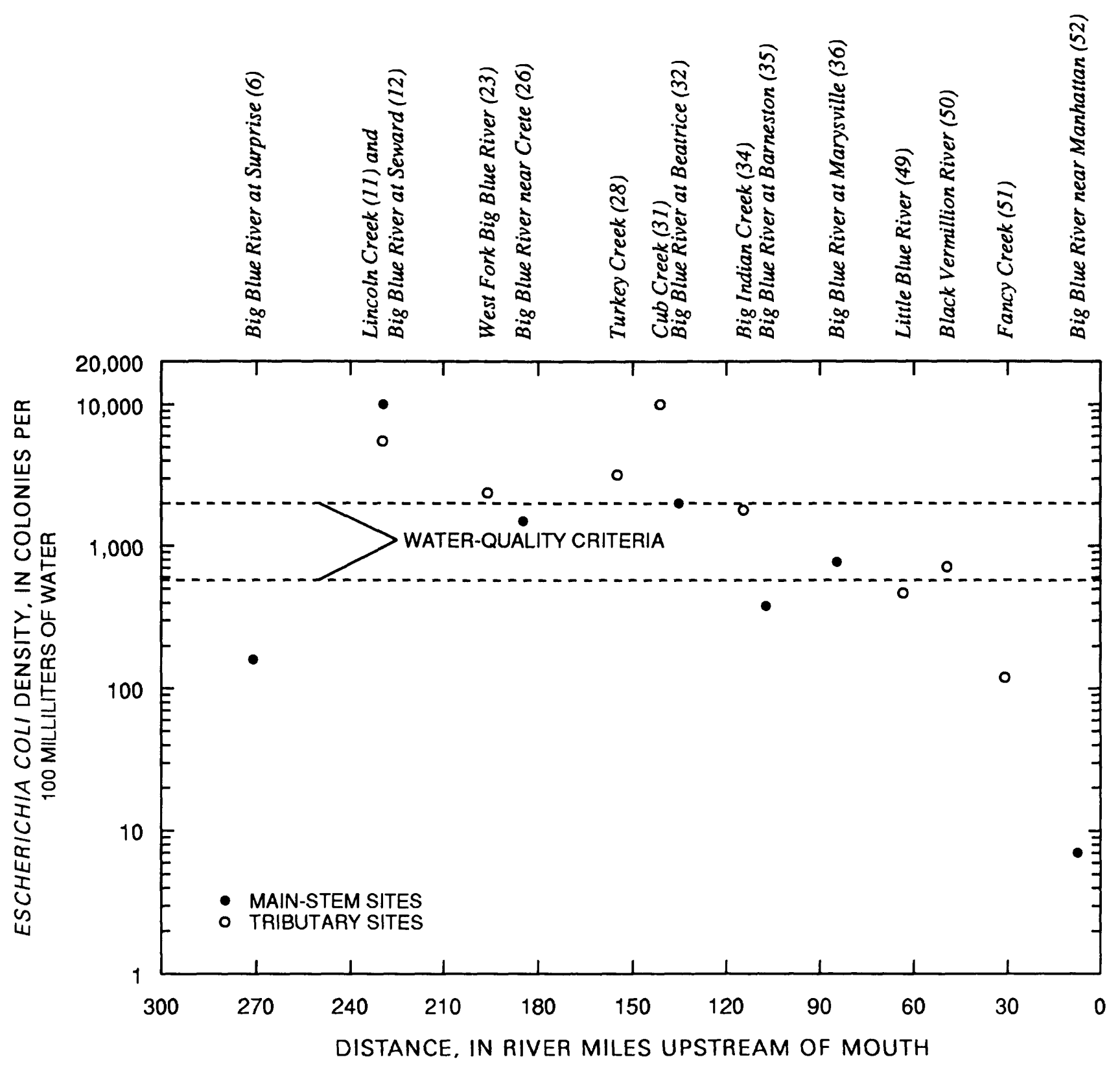

Figure 15. Distribution of Escherichia coli densities in water from the Big Blue River and its major tributaries for synoptic survey of July $24-29,1988$. [Number in parentheses is synoptic-sampling site number. Water-quality criteria established by U.S. Environmental Protection Agency (1986) and Kansas Department of Health and Environment (Fromm and Wilk, 1988).]

cate that nonpoint-source contamination is minimal in this part of the subbasin. The Little Blue River near Fairbury, Nebr. (site 44), is approximately 2 mi downstream of the Fairbury municipal wastewatertreatment plant. This plant has a permitted discharge of $0.65 \mathrm{Mgal} / \mathrm{d}$ and is probably responsible for the increase in $E$. coli density $(1,200 \mathrm{col} / 100 \mathrm{~mL})$ over that determined at site 40 . Nonpoint-source contamination may be responsible for the relatively large E. coli density $(5,500 \mathrm{col} / 100 \mathrm{~mL})$ in Rose Creek near 


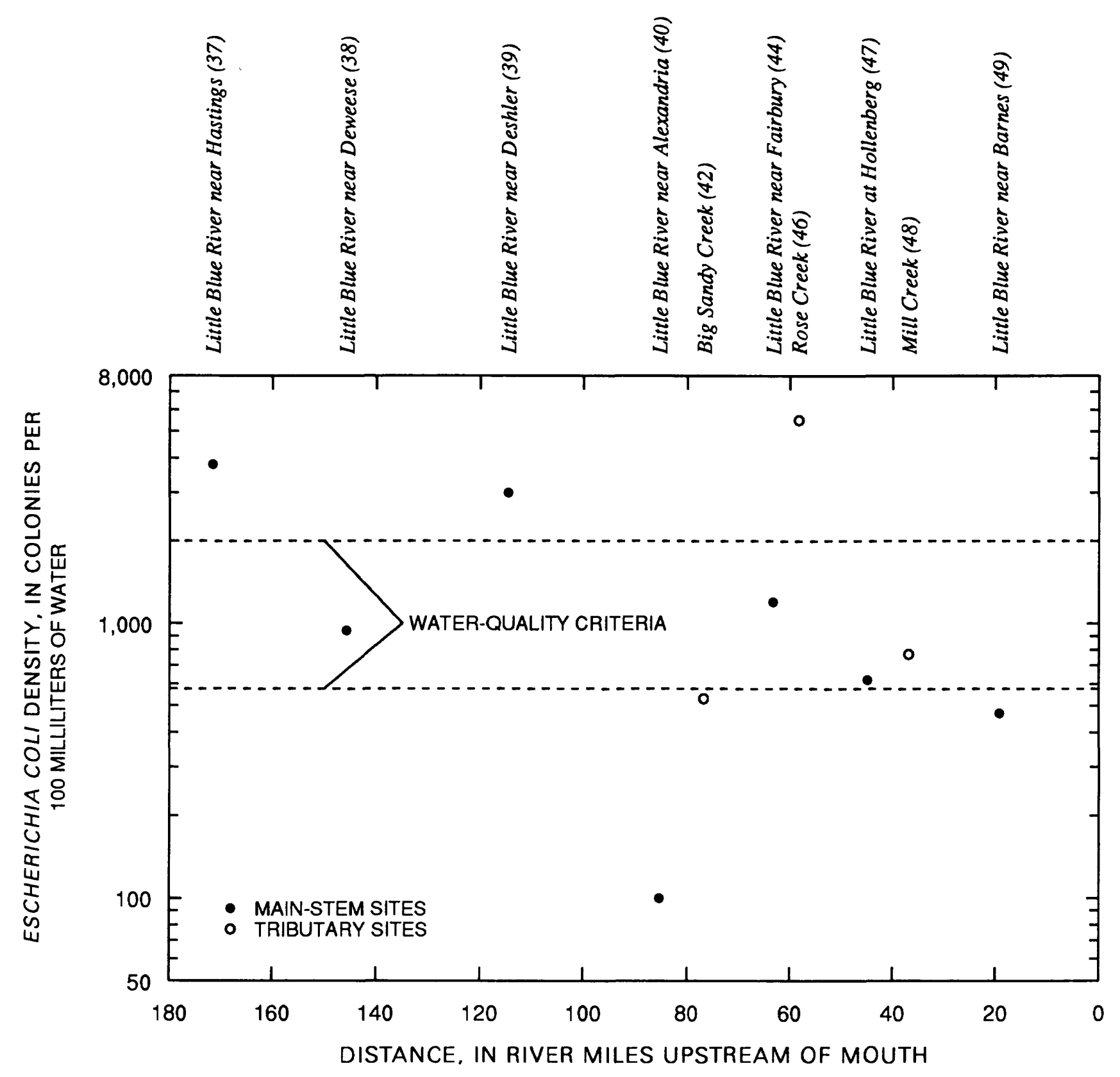

Figure 16. Distribution of Escherichia coli densities in water from the Little Blue River and its major tributaries for synoptic survey of July $24-29,1988$. [Number in parentheses is synoptic-sampling site number. Water-quality criteria established by U.S. Environmental Protection Agency (1986) and Kansas Department of Health and Environment (Fromm and Wilk, 1988).]

Fairbury (site 46). Although there are two wastewatertreatment plants upstream from site 46 , the closest is more than 20 stream mi upstream and has a permitted discharge of only $0.01 \mathrm{Mgal} / \mathrm{d}$. Therefore, the contribution of these wastewater-treatment plants to the
E. coli density determined at site 46 is thought to be minimal.

Downstream of Fairbury, Nebr., E. coli densities gradually decline on both main-stem and tributary streams. Although not shown in figure 16, the largest 
E. coli density $(30,000 \mathrm{col} / 100 \mathrm{~mL})$ in the Little Blue River subbasin was determined in Big Sandy Creek near Davenport, Nebr. (site 41). This density may be point-source dominated. Although three small wastewater-treatment plants are located upstream of site 41 ( 3 to 20 stream mi), their proximity and combined permitted discharge $(0.13 \mathrm{Mgal} / \mathrm{d})$ are probably insufficent to produce the density determined at site 41 .

Of the 27 sites sampled for $E$. coli in the Kansas River subbasin, only Elk Creek near Larkinburg, Kans. (site 73), a tributary to the Delaware River, exceeded the $576-\mathrm{col} / 100 \mathrm{~mL}$ criterion for primary contact. That $1,000-\mathrm{col} / 100 \mathrm{~mL}$ density did not exceed the $2,000-\mathrm{col} / 100 \mathrm{~mL}$ criterion for other than primary contact. This relatively large density may result from the $0.49-\mathrm{Mgal} / \mathrm{d}$ permitted discharge from the Holton, Kans., wastewater-treatment plant, located approximately 8 stream mi upstream from site 73 .

A downstream-ordered distribution of $E$. coli densities for the main stem of the Kansas River and major tributaries as determined during the July 1988 synoptic survey is shown in figure 17. Densities of $E$. coli in the Kansas River generally appear to be pointsource dominated during stable base-flow conditions. The most upstream site, Kansas River at Fort Riley, Kans. (site 1), likely is affected by the Junction City, Kans., wastewater-treatment plant about 2 stream mi upstream. The Junction City wastewater-treatment plant has permitted discharge exceeding $1.0 \mathrm{Mgal} / \mathrm{d}$. E. coli density at site 1 was $210 \mathrm{col} / 100 \mathrm{~mL}$. At Manhattan (site 5), E. coli density was $96 \mathrm{col} / 100 \mathrm{~mL}$, which may indicate that the distance between sites 1 and 5 is a reach of $E$. coli die off; however, because of differences in sampling times and flow rates (table 4), this is difficult to confirm. Near Belvue, Kans. (site 58 ), approximately 35 stream mi downstream of site 5 , density of $E$. coli had decreased to $48 \mathrm{col} / 100 \mathrm{~mL}$ despite the 2.74-Mgal/d permitted discharge from the Manhattan wastewater-treatment plant. The smaller density at site 58 was probably the result of dilution by a large release $\left(1,870 \mathrm{ft}^{3} / \mathrm{s}\right)$ from Tuttle Creek Lake via the Big Blue River. Downstream of the confluence of the Big Blue and Kansas Rivers, Tuttle Creek Lake water comprised 89 percent of the flow in the Kansas River. Density of $E$. coli in the release water from Tuttle Creek Lake was $7 \mathrm{col} / 100 \mathrm{~mL}$ (site 52), which provided a substantial dilution effect in the Kansas River.
The largest densities of $E$. coli in the Kansas River occur downstream of large municipal wastewater treatment-plant discharge points. Site 76 and site 78 are downstream of the $14.3 \mathrm{Mgal} / \mathrm{d}$ permitted discharge at Topeka, Kans., and the $5.3 \mathrm{Mgal} / \mathrm{d}$ permitted discharge at Lawrence, Kans., respectively. The 390-col/100 mL density determined at the downstream-most site, Kansas River at Kansas City, Kans. (site 91), probably can be attributed to wastewater discharges from several large and small treatment plants in Johnson County, Kans.

Point-source discharges from municipal wastewater-treatment plants have a substantial effect on $E$. coli densities at several of the sites in the Big Blue River and Little Blue River subbasins. During the time of the synoptic surveys conducted for this study, none of the wastewater-treatment plants in the study unit disinfected (chlorinated) effluent before discharge into receiving streams. The drainage areas of all sampling sites in these subbasins contained one to several treatment plants; however, most of these plants serve small communities and typically have permitted-discharge rates of less than $0.10 \mathrm{Mgal} / \mathrm{d}$. Therefore, their potential effect may be limited to sites within several stream miles of the discharge point and may have only minimal effect at sites farther downstream. The effect of larger facilities, greater than $1.0 \mathrm{Mgal} / \mathrm{d}$, is most evident at the West Fork Big Blue River near Hastings, Nebr. (site 16), where an E. coli density of $260,000 \mathrm{col} / 100 \mathrm{~mL}$ was determined. Site 16 is approximately $2 \mathrm{mi}$ downstream from the Hastings municipal wastewater-treatment plant, which has a permitted discharge of $2.75 \mathrm{Mgal} / \mathrm{d}$. Site 16 also was characterized by DO concentrations in violation of 1-day minimum criteria (table 3 ) and by large nutrient concentrations (table 8) as previously explained in the "Dissolved-Oxygen Concentrations" section of this report. Other sites in the Big Blue River and Little Blue River systems that appear to have E. coli densities affected by close proximity to pointsource discharges include Lincoln Creek near Aurora, Nebr. (site 9); School Creek near Sutton, Nebr. (site 18); Beaver Creek near York, Nebr. (site 21); Big Blue River near Crete, Nebr. (site 26); Big Sandy Creek near Davenport, Nebr. (site 41); and Little Blue River near Fairbury, Nebr. (site 44). 


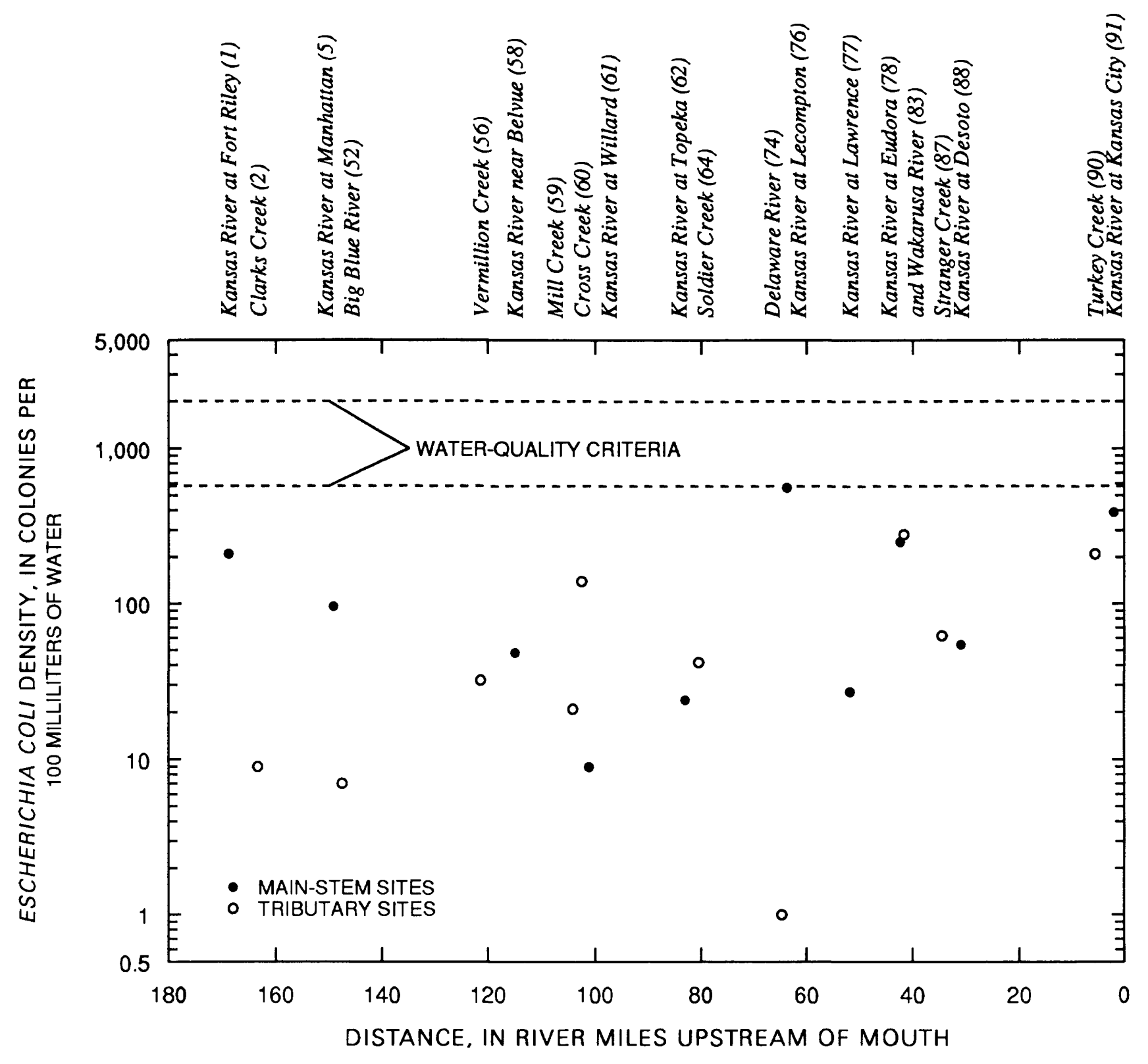

Figure 17. Distribution of Escherichia coli densities in water from the Kansas River and its major tributaries for synoptic survey of July 24-29, 1988. [Number in parentheses is synoptic-sampling site number. Water-quality criteria established by U.S. Environmental Protection Agency (1986) and Kansas Department of Health and Environment (Fromm and Wilk, 1988).]

\section{Regional Patterns}

Previously presented information indicate regional patterns in $E$. coli densities. These regional patterns are most evident in a comparison of median values (50th percentile), figure 18, of the July 1988 samples from the 19 sites in the Big Blue River subbasin $(2,400 \mathrm{col} / 100 \mathrm{~mL})$, the 11 sites in the Little Blue River subbasin $(940 \mathrm{col} / 100 \mathrm{~mL})$, and the 27 sites in the Kansas River subbasin $(88 \mathrm{col} / 100 \mathrm{~mL})$. The 


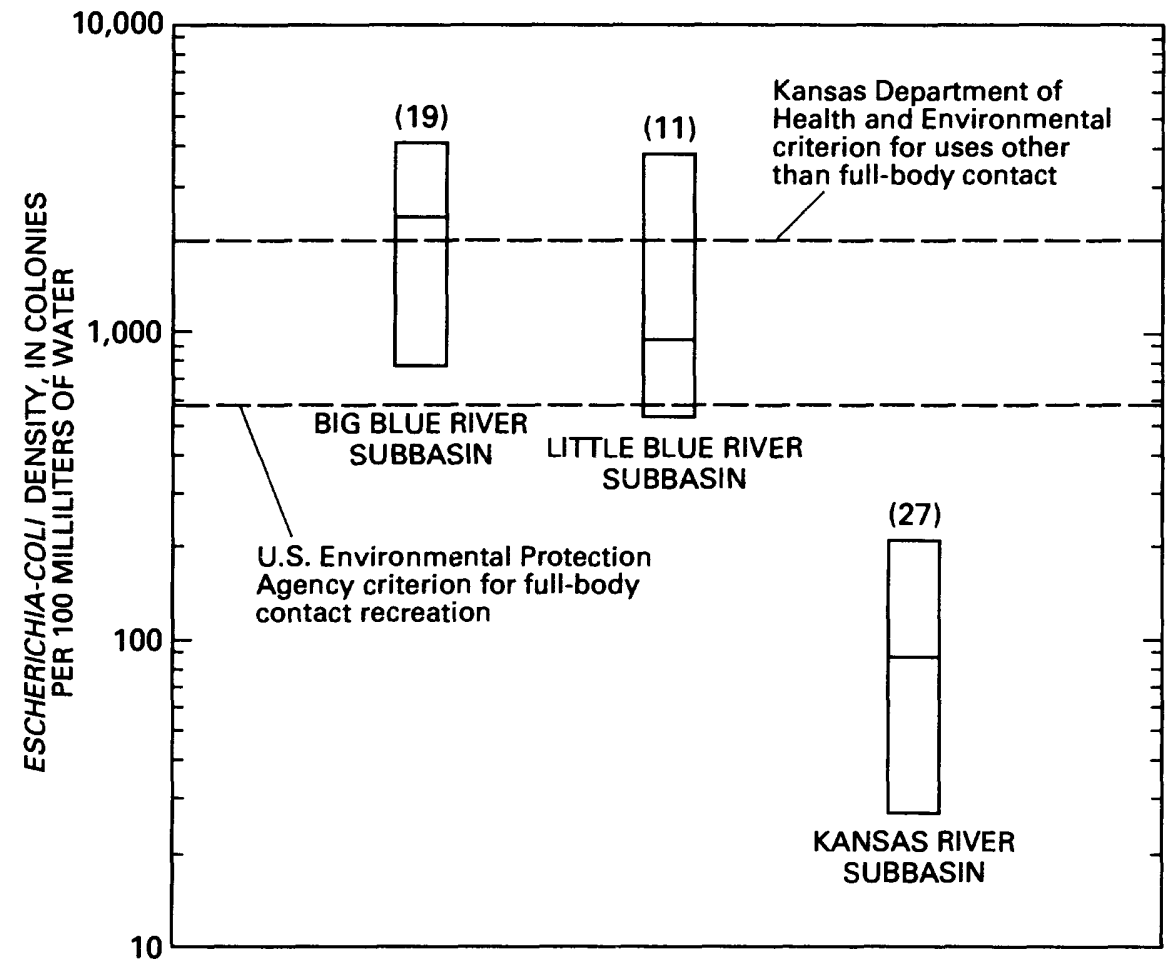

\section{EXPLANATION}

(11) 75th PERCENTILE

NUMBERS IN PARENTHESES ABOVE BOXPLOT ARE NUMBER OF SAMPLES

Figure 18. Comparison of the 25th, 50th (median), and 75th percentiles of Escherichia coli densities in the Big Blue River, Little Blue River, and Kansas River subbasins for synoptic survey of July 24-29, 1988 [water-quality criteria established by U.S. Environmental Protection Agency, 1986, and Kansas Department of Health and Environment (Fromm and Wilk, 1988)].

median $E$. coli density in samples from the Big Blue River subbasin was 2.6 times larger than in samples from the Little Blue River subbasin and 27.3 times larger than in samples from the Kansas River subbasin.

Although point-source discharges may be responsible for large $E$. coli densities at specific sites during base-flow conditions and $E$. coli may be carried to some sites farther downstream, it appears that additional factors may be responsible for the generally larger $E$. coli densities in the Big Blue River and Little Blue River subbasins compared to those determined in the Kansas River subbasin. The Kansas River subbasin contains many wastewater-treatment plants with large and small permitted discharges located on large and small streams, similar to the situation in the Big Blue River and Little Blue River subbasins. However, with only one exception, $E$. coli densities in the Kansas River subbasin were less than water-quality criteria during the July 1988 survey. The large $E$. coli densities in the Big Blue River and Little Blue River subbasins probably is most attributable to the production of domestic livestock.

Domestic livestock production may increase $E$. coli densities in streams in several ways: (1) direct access to streams used as a water source by the livestock, (2) overland runoff from pastures and feedlots, (3) ground-water discharge in areas where feedlots 
may be in close proximity to streams, and (4) runoff as a result of rainfall or irrigation on cropland where manure was applied as a fertilizer or soil amendment.

Livestock may contribute fecal material and $E$. coli directly to streams when those streams are used as a water source. This may occur more frequently in areas where livestock are pastured instead of confined to feedlots. Direct access to streams, however, may not be a major cause of E. coli loading. The Kansas River subbasin has more than twice the percentage of pasture and rangeland than the other major subbasins examined in this report (fig. 5). However, it had the smallest median $E$. coli density $(88 \mathrm{col} / 100 \mathrm{~mL})$ of any subbasin.

Feedlots may contribute to the occurrence of $E$. coli in streams because of uncontrolled surface runoff or because $E$. coli is transported downward by infiltrating rain to shallow ground water and then laterally to nearby streams. As previously described in the "Livestock Production" section of this report, counties with the largest percentage of cattle in confined feeding operations and the greatest densities of cattle and hogs are located in the headwater or middle-stream reaches of the Big Blue, West Fork Big Blue, and Little Blue Rivers (figs. 7 and 8).

One stream that may indicate the effect of livestock feeding and production operations is Lincoln Creek (fig. 1) in the extreme northern part of the study unit. Three sampling sites (sites 9, 10, and 11, table 4) were located on Lincoln Creek. The upstream-most site, near Aurora, Nebr. (site 9), is approximately $1.25 \mathrm{mi}$ downstream of the Aurora wastewatertreatment facility, which probably is the main source of the $2,600 \mathrm{col} / 100 \mathrm{~mL}$ of $E$. coli measured at site 9 . The instantaneous load of $E$. coli organisms at site 9 was 250,000 organisms per second (org/s). Instantaneous load is computed by multiplying $E$. coli density $(\mathrm{col} / 100 \mathrm{~mL})$ by streamflow rate $\left(\mathrm{ft}^{3} / \mathrm{s}\right)$ by a unit conversion factor of 283.2 based on the assumption that one colony developed from one $E$. coli organism. At the site near York, Nebr. (site 10), which is 42 stream mi downstream of site $9, E$. coli density had decreased to $1,900 \mathrm{col} / 100 \mathrm{~mL}$. However, instantaneous load of $E$. coli had increased by a factor of almost 18 to $4,400,000 \mathrm{org} / \mathrm{s}$. There are no known point-source discharges between site 9 and 10. At site 11 near Seward, Nebr., 47.3 stream mi downstream of site $10, E$. coli density had increased to $5,500 \mathrm{col} / 100$ $\mathrm{mL}$, and instantaneous load had increased by more than an order of magnitude to $47,000,000 \mathrm{org} / \mathrm{s}$. The only known point-source discharge between sites 10 and 11 is the $0.02 \mathrm{Mgal} / \mathrm{d}$ permitted discharge from the Gresham, Nebr., wastewater-treatment plant located on a tributary to Lincoln Creek many miles upstream of Seward.

From these results it appears that large numbers of bacteria are entering Lincoln Creek between sites 9 and 11 from nonpoint sources. The intensity of feedlot operations and large cattle and hog densities in the Lincoln Creek Basin probably account for a large part of the E. coli load in Lincoln Creek. This load of $E$. coli may be a result of cattle and hogs wading in Lincoln Creek or its tributaries, $E$. coli entering streams with ground water affected by feedlot operations, or the load may be an artifact of recent rainfall runoff (between 5-14 days before the survey, table 5).

Gary and others (1983) found that when at least 150 cattle were grazed in a pasture in central Colorado, fecal coliform densities in stream water bisecting the pasture were significantly larger than those in an adjacent, ungrazed pasture. After removal of cattle or when 40 head of cattle were grazing, bacterial counts decreased to levels similar to the ungrazed pasture. Also, they concluded that about 5 percent of the total manure produced by cattle contributed to contamination of the stream. Obviously, this percentage depends upon factors such as soil type, soil porosity, topography, land cover, and rainfall characteristics.

Stephenson and Street (1978) determined that the occurrence of fecal coliforms at stream sites in a rangeland watershed in southwest Idaho was related directly to the presence of cattle on summer range and winter pastures. Fecal coliform counts were found to increase soon after cattle were pastured and remained large for several months after cattle were removed.

Feedlot operations may have an indirect effect on stream water quality. In any confined feeding operation (cattle or hogs), large quantities of manure will be produced creating a disposal problem. One disposal solution is to apply the manure to cropland as a lowgrade fertilizer or soil amendment. This application may be in late fall after harvest or early spring before planting. Subsequent rainfall or irrigation runoff may transport organic material and $E$. coli into nearby streams. If this practice is widespread, it may have a substantial effect on $E$. coli densities in streams in intensively irrigated areas.

In the Big Blue River and Little Blue River subbasins, percentages of land used for irrigated row 
crops were 25.1 percent and 18.5 percent, respectively (fig. 5). These percentages generally are much larger for the upper part of each subbasin, where $E$. coli densities were largest, as most of the range or pastureland shown in figure 5 occurs in the Plains Border and Dissected Till Plains physiographic sections (fig. 2) south of the Kansas-Nebraska border. In contrast, irrigated row crops in the Kansas River subbasin accounted for much less than 1 percent (too small to show in fig. 5), whereas in the Delaware River subbasin irrigated row crops totaled only 1.5 percent of the total land use. This information indicates that the possibility of irrigation-water runoff affecting $E$. coli densities would be greatest for the Big Blue River and Little Blue River subbasins. Irrigation-water runoff was observed entering Turkey Creek immediately upstream of the sampling site near Wilber, Nebr. (site 28), during the July 1988 synoptic survey. Considering the extent of irrigation in the Big Blue River and Little Blue River subbasins, many other streams in these subbasins may have been receiving irrigation-water runoff.

Further evidence of the effect of livestock production on bacterial densities is presented in Jordan and Stamer (1991). In examining historical bacteriological data, they reported moderate fecal coliform and large fecal streptococci densities in water from Lincoln Creek near Seward, Nebr. (site 11). They indicated that, on the basis of a study done by Geldreich and Kenner (1969), when fecal streptococci were present in greater numbers than fecal coliform bacteria, the originating source of fecal material was "farm animals, dogs, cats, and various wild animals." When the converse situation occurs, the originating source is human. In addition to the potential contribution of bacteria from livestock production, irrigation-water runoff, as previously discussed, could contribute to bacteria loading along Lincoln Creek. With the data currently available, it is impossible to quantify the contribution of either source; however, given the extent of livestock production, it is believed that this activity is the source of most of the nonpointsource loading of bacteria. The situation on Lincoln Creek is not unique and also could apply to many other streams in the Big Blue River and Little Blue River subbasins.

In July 1989, 36 of the 57 sites sampled in July 1988 were resampled in an attempt to verify the distributional pattern and magnitude of $E$. coli densities determined in July 1988. Sites sampled in July 1989 and $E$. coli densities are listed in table 4. Making this verification survey comparable to the data collected in 1988 required conditions similar to those during the July 1988 survey; however, as previously discussed, precipitation (table 5) and hydrologic conditions (table 6) during the July 1989 sampling were, in many instances, very dissimilar from those in July 1988. With the exception of Big Indian Creek near Wymore, Nebr. (site 34), streamflow at all sampling sites in the Big Blue and Little Blue River subbasins upstream of Tuttle Creek Lake was affected by overland runoff from recent rain. This is especially true in the Little Blue River subbasin (sites 37-49) where streamflows were as much as two orders of magnitude larger (site 38) than those observed in July 1988.

Densities of $E$. coli determined in July 1989 generally were larger than those in July 1988 and ranged from $5 \mathrm{col} / 100 \mathrm{~mL}$ in the Delaware River below Perry Dam, Kans. (site 74), to $790,000 \mathrm{col} / 100 \mathrm{~mL}$ in the West Fork Big Blue River near Hastings, Nebr. (site 16). These two sites also had the minimum and maximum densities, respectively, of all sites sampled in July 1988. The larger densities determined in July 1989 appear to be the result of nonpoint-source contamination from overland runoff caused by rainfall during the 14 days prior to survey. The largest densities in July 1989 were at sites located in the Big Blue and Little Blue River subbasins, a situation similar to that observed in July 1988.

Although the July $1989 E$. coli data set is not adequate for verifying the results of the stable, lowflow conditions of July 1988, it does provide insight into the relation between runoff and bacterial densities. This relation is most evident in the Little Blue River subbasin, particularly at main-stem sites where flows were many times larger than those observed in July 1988 . In July 1988 , the median E.coli density at main-stem Little Blue River sites was $940 \mathrm{col} / 100 \mathrm{~mL}$, whereas in July 1989 the median density was $17,000 \mathrm{col} / 100 \mathrm{~mL}$. The intense livestock production in the Little Blue and Big Blue River subbasins is a major contributor to fecal contamination of streams receiving overland runoff in these areas.

Densities of $E$. coli in the Kansas River subbasin were considerably larger in July 1989 than in July 1988 but not at the magnitude observed in the Little Blue and Big Blue River subbasins. The larger densities in July 1989 were probably the result of nonpoint-source contribution from overland runoff caused by considerable rainfall during and just before 
the start of sampling (table 5). The largest densities determined in the Kansas River subbasin were at main-stem sites downstream of Topeka (sites 76, 78, and 88 ), which received nearly 3 in. of rain during this period. The $10,000-\mathrm{col} / 100 \mathrm{~mL}$ density determined at Kansas River at Lecompton, Kans. (site 76), probably represents a combination of nonpoint sources from overland runoff and discharge from the Topeka wastewater-treatment plant.

\section{SUMMARY}

In 1986, the Congress appropriated funds for the U.S. Geological Survey to test and refine concepts for a National Water-Quality Assessment (NAWQA) Program. The 15,300- $\mathrm{mi}^{2}$ lower Kansas River Basin in Kansas and Nebraska was one of four pilot studies in this program. This report describes the sanitary quality of streams in the lower Kansas River Basin. Sanitary quality is evaluated on the basis of concentrations of dissolved oxygen (DO) and densities of a fecalindicator bacterium, Escherichia coli ( $E$. coli).

Sixty-one surface-water sampling sites were chosen for this investigation. Criteria for site selection included long-term, fixed-station sampling locations, locations of potential degradation in sanitary quality, locations on streams representing areas with reasonably homogeneous land use, sites at the confluence of major tributaries, and additional sites on the main stem Kansas River and major tributary streams to provide a resolution of no more than 50 river mi between sites. Synoptic surveys were conducted at these sites in July 1988, November 1988, March 1989, and May 1989, to define the concentrations, and diel and seasonal variability in concentrations of DO. Synoptic surveys were conducted in July 1988 and July 1989 to define densities of $E$. coli. Ancillary data included measurements of specific conductance, $\mathrm{pH}$, water temperature, barometric pressure, and concentrations of nutrients, total organic carbon, chlorophyll, and suspended sediment. Surveys generally were conducted during stable-flow, dry-weather conditions.

To define the maximum potential for DO deficiencies, a synoptic survey was conducted in July 1988 when water temperatures were expected to be near annual maximums and streamflows near annual minimums. Because DO fluctuates as a result of daytime photosynthesis (production of oxygen) and nighttime respiration (consumption of oxygen) by algae and aquatic plants, 31 sites were sampled within about 2 hours before dawn when DO concentrations were anticipated to be at minimum in the diel cycle. Of these 31 sites, 5 had DO concentrations less than the U.S. Environmental Protection Agency (USEPA) 5.0-mg/L, 1-day minimum warmwater criterion for early life stages, and 4 of these 5 predawn concentrations were less than the USEPA $3.0-\mathrm{mg} / \mathrm{L}$ criterion for all other life stages. These five sites were Lincoln Creek near Aurora, Nebr., West Fork Big Blue River near Hastings, Nebr., Delaware River near Fairview, Kans., Stranger Creek at Easton, Kans., and Turkey Creek at Kansas City, Kans. The small DO concentrations at the sampling sites on Lincoln Creek, West Fork Big Blue River, and Turkey Creek were mainly the result of upstream municipal wastewater discharges. Small DO concentrations in Stranger Creek and the Delaware River were mainly the result of algal respiratory demands coupled with reduced physical reaeration associated with extreme low flow.

In addition to those sites identified in the July 1988 synoptic survey with DO concentrations less than criteria, concentrations at four additional sites identified in subsequent surveys were less than criteria: Beaver Creek near York, Nebr., on May 31, 1989 (4.9 mg/L); Cross Creek at Rossville, Kans., on June 1, 1989 (4.7 mg/L); Wakarusa River near Berryton, Kans., on May 31, 1989 (4.5 mg/L); and Wakarusa River near Lawrence, Kans., on November $16,1988(2.7 \mathrm{mg} / \mathrm{L})$. The small DO concentration at the site on Beaver Creek appeared to be the result of municipal wastewater discharge, whereas the small concentrations in Cross Creek and Wakarusa River near Berryton, Kans., probably were caused by algal respiratory demands coupled with reduced physical reaeration associated with extreme low flow. The small DO concentration at Wakarusa River near Lawrence, Kans., was possibly the result of localized runoff carrying oxygen-demanding material into the stream.

Concentrations of DO in violation of water-quality criteria are localized occurrences and do not reflect regional patterns in DO. Generally, the most severe DO deficiencies are the result of discharges from municipal wastewater-treatment plants into small tributary streams with inadequate dilution or assimilation capacity. Algal respiratory demand in combination with reduced physical reaeration associated with extreme low flow probably also contributes to temporary, localized deficiencies. Comparisons among 
mean predawn DO concentrations at sites on the Big Blue River, Little Blue River, and Kansas River, showed little appreciable difference between the streams $(6.23,6.60$, and $6.36 \mathrm{mg} / \mathrm{L}$, respectively). Of all DO concentrations determined (392) in conjunction with all synoptic surveys, nine determinations (2.3 percent) were less than water-quality criteria.

Diel variability in DO concentrations was measured at 11 sites in the study unit. DO determinations were made at 4- or 6-hour intervals during an approximately 24 -hour period. Concentrations of DO less than the $5.0-\mathrm{mg} / \mathrm{L}$ criterion were not observed at any of the diel-study sites. On the basis of these diel studies, it appears that diel DO fluctuations in streams not affected by localized problems such as pointsource discharges are basically a function of photosynthesis and respiration.

Densities of $E$. coli were determined at 57 surfacewater sampling sites during the synoptic survey in July 1988. Results indicate large regional differences in $E$. coli densities within the study unit. The median $E$. coli density in the Big Blue River subbasin was $2,400 \mathrm{col} / 100 \mathrm{~mL}$ and ranged from 120 to $260,000 \mathrm{col} / 100 \mathrm{~mL}$. The median $E$. coli density in the Little Blue River subbasin was $940 \mathrm{col} / 100 \mathrm{~mL}$ and ranged from 100 to $30,000 \mathrm{col} / 100 \mathrm{~mL}$. The median $E$. coli density in the Kansas River subbasin was $88 \mathrm{col} / 100 \mathrm{~mL}$ and ranged from less than 1 to $1,000 \mathrm{col} / 100 \mathrm{~mL}$.

Of the 19 sites sampled in the Big Blue River subbasin upstream of Tuttle Creek Lake, stream water at 16 ( 84 percent) of those sites exceeded the USEPA criterion for $E$. coli densities $(576 \mathrm{col} / 100 \mathrm{~mL})$ for infrequently used full-body contact recreation, and 10 sites ( 53 percent) exceeded the $2,000-\mathrm{col} / 100 \mathrm{~mL}$ fecal coliform criterion for uses other than full-body contact established by the Kansas Department of Health and Environment. Eight ( 73 percent) of the 11 sites sampled in the Little Blue River subbasin exceeded the $576-\mathrm{col} / 100 \mathrm{~mL}$ full-body criterion, and 4 sites (36 percent) exceeded the $2,000-\mathrm{col} / 100 \mathrm{~mL}$ fecal coliform criterion for other than full-body contact. In the Kansas River subbasin, the $576-\mathrm{col} / 100 \mathrm{~mL}$ criterion was exceeded at only one site.

Point-source discharges from municipal wastewater-treatment plants mainly were responsible for the large $E$. coli densities measured at specific sites in the study unit; however, nonpoint-source contamination probably was responsible for the generally larger E. coli densities in the Big Blue and Little Blue
River subbasins compared to those in the Kansas River subbasin. This nonpoint-source contamination may have been the result of greater livestock production in the Big Blue River and Little Blue River subbasins compared to that in the Kansas River subbasin. More grazing and a greater number of confined feeding operations could increase $E$. coli densities in streams in several ways: (1) direct access to streams used as a water source by livestock, (2) overland runoff from pastures and confinement areas, (3) groundwater discharge from confinement areas in close proximity to streams, and (4) rainfall or irrigation runoff from cropland where manure was applied as fertilizer or soil amendment.

\section{REFERENCES CITED}

Bayne, C.K., 1975, General availability of ground water and normal annual precipitation in Kansas: Kansas Geological Survey Map Series M-4A, 1 sheet, scale 1:500,000.

Britton, L.J., and Greeson, P.E., 1987, Methods for collection and analysis of aquatic biological and microbiological samples: U.S. Geological Survey Techniques of Water-Resources Investigations, book 5, Laboratory analysis, chap. A4, 363 p.

Brown, Eugene, Skougstad, M.W., and Fishman, M.J., 1970, Methods for collection and analysis of water samples for dissolved minerals and gases: U.S. Geological Survey Techniques of WaterResources Investigations, book 5, chap. A1, $160 \mathrm{p}$.

Buchanan, T.J., and Somers, W.P., 1976, Discharge measurements at gaging stations: U.S. Geological Survey Techniques of Water-Resources Investigations, book 3, chap. A8, 65 p.

Doran, J.W., Schepers, J.S., and Swanson, N.P., 1981, Chemical and bacteriological quality of pasture runoff: Journal of Soil and Water Conservation, v. 36, no. 3, p. 166-171.

Dufour, A.P., Strickland, E.R., and Cabelli, V.J., 1981, Membrane filter method for enumerating Escherichia coli: Applied and Environmental Microbiology, v. 41, no. 5, p. 1152-1158.

Ellis, M.J., 1981, Analysis of stream-aquifer system interrelationships in the Big Blue and Little Blue River Basins in Gage and Jefferson Counties, Nebraska: U.S. Geological Survey Water-Resources Investigations 81-29, $49 \mathrm{p}$.

Fader, S.W., 1974, Ground water in the Kansas River valley, Junction City to Kansas City, Kansas: Kansas Geological Survey Bulletin 206, part 2, 12 p. 
Fallon, J.D., and McChesney, J.A., 1993, Surface-waterquality assessment of the lower Kansas River Basin, Kansas and Nebraska-Project data, November 1986 through April 1990: U.S. Geological Survey Open-File Report 93-51, 594 p.

Fenneman, N.M., 1946, Physical divisions of the United States: Washington, D.C., U.S. Geological Survey special map, 1 sheet, scale 1:7,000,000.

Fishman, M.J., and Friedman, L.C., eds., 1989, Methods for determination of inorganic substances in water and fluvial sediments: U.S. Geological Survey Techniques of Water-Resources Investigations, book 5, chap. A1, $545 \mathrm{p}$.

Fromm, Carla, and Wilk, Sally, eds., 1988, Kansas water quality assessment 1986-1987: Topeka, Kansas Department of Health and Environment 305 (b) Report, 144 p.

Gary, H.L., Johnson, S.R., and Ponce, S.L., 1983, Cattle grazing impact on surface water quality in a Colorado front range stream: Journal of Soil and Water Conservation, v. 38, no. 2, p. 124-128.

Geldreich, E.E., and Kenner, B.A., 1969, Concepts of fecal streptococci in stream pollution: Journal of the Water Pollution Control Federation, v. 41, no. 8, p. R336R352.

Guy, H.P., 1977, Laboratory theory and methods for sediment analysis: U.S. Geological Survey Techniques of Water-Resources Investigations, book 5, chap. C1, $50 \mathrm{p}$.

Guy, H.P., and Norman, V.W., 1970, Field methods for measurement of fluvial sediment: U.S. Geological Survey Techniques of Water-Resources Investigations, book 3, chap. C2, $59 \mathrm{p}$.

Hagedorn, C., Hansen, D.T., and Simonson, G.H., 1978, Survival and movement of fecal indicator bacteria in soil under conditions of saturated flow: Journal of Environmental Quality, v. 7, no. 1, p. 55-59.

Hines, W.G., McKenzie, S.W., Richert, D.A., and Rinella, F.A., 1977, Dissolved-oxygen regimen of the Willamette River, Oregon, under conditions of basinwide secondary treatment: U.S. Geological Survey Circular 715-I, $52 \mathrm{p}$.

Jordan, P.R., and Stamer, J.K., eds., 1991, Surface-waterquality assessment of the lower Kansas River Basin, Kansas and Nebraska-Analysis of available data through 1986: U.S. Geological Survey Open-File Report 91-75, 172 p.

Kansas State Board of Agriculture and U.S. Department of Agriculture, 1988-89, Kansas farm facts: Topeka, Kans. (published annually).

Kress, M., and Gifford, G.F., 1984, Fecal coliform release from cattle fecal deposits: Water Resources Bulletin, v. 20, no. 1, p. 61-66.

Lim, C.H., and Flint, K.P., 1989, Effects of nutrients on the survival of Escherichia coli in lake water: Journal of Applied Bacteriology, v. 66, no. 6, p. 559-569.
McKinney, R.E., 1962, Microbiology for sanitary engineers: New York, McGraw-Hill Inc., 153 p.

National Oceanic and Atmospheric Administration, 1951-80, Climatological data, Kansas, annual summary: Asheville, N.C., National Climatic Center (published annually).

1988-89a, Climatological data, Kansas, monthly summary: Asheville, N.C., National Climatic Center (published monthly).

1988-89b, Climatological data, Nebraska, monthly summary: Asheville, N.C., National Climatic Center (published monthly).

Nebraska Department of Agriculture and U.S. Department of Agriculture, 1989, Nebraska agricultural statistics: Lincoln, Nebr., 154 p.

Nebraska Department of Environmental Control, 1986, 1986 Nebraska water quality report: Lincoln, Neb., $212 \mathrm{p}$.

Reid, G.K., and Wood, R.D., 1976, Ecology of inland waters and estuaries: New York, D. Van Nostrand Co., p. 210-213, 215.

Robbins, W.W., Weier, T.E., and Stocking, C.R., 1964, Botany-An introduction to plant science: New York, John Wiley and Sons, Inc., p. 57.

Seaber, P.R., Kapinos, F.P., and Knapp, G.L., 1984, State hydrologic unit maps: U.S. Geological Survey Open-File Report 84-708, 198 p.

Seyfried, P.L., Brown, N.E., Cherwinsky, C.L., Jenkins, G.D., and Cotter, D.A., 1984, Impact of sewage treatment plants on surface waters: Canadian Journal of Public Health, v. 75, no. 1, p. 25-31.

Stephenson, G.R., and Street, L.V., 1978, Bacterial variations in streams from a southwest Idaho rangeland watershed: Journal of Environmental Quality, v. 7, no. 1, p. 150-157.

U.S. Environmental Protection Agency, 1985, Test methods for Escherichia coli and Enterococci in water by the membrane filter procedure: U.S. Environmental Protection Agency, EPA 600/4-85/076, 12 p. 1986, Quality criteria for water 1986: U.S. Environmental Protection Agency, EPA 440/5-86-001, unnumbered pages.

U.S. Soil Conservation Service, 1987, National resources inventory: Washington, D.C., U.S. Soil Conservation Service, digital data.

Wershaw, R.L., Fishman, M.J., Grabbe, R.R., and Lowe, L.E., eds., 1983, Methods for the determination of organic substances in water and fluvial sediments: U.S. Geological Survey Techniques of Water-Resources Investigations, book 5, Laboratory analysis, chap. A3, $173 \mathrm{p}$.

Williams, D.L., and Barker, B.L., 1974, Kansas land-use map, summer 1973: Kansas Department of Economic Development, 1 sheet, scale 1:1,000,000. 
Table 8. Results of ancillary chemical analyses of water samples collected July 1988 through June 1989 in the lower Kansas River Basin

[mg/L, milligrams per liter, $\mu \mathrm{g} / \mathrm{L}$, micrograms per liter; <, less than; --, no data]

\begin{tabular}{|c|c|c|c|c|c|c|c|c|c|c|}
\hline $\begin{array}{c}\text { Site } \\
\text { map- } \\
\text { index } \\
\text { number } \\
\text { (fig. 10) }\end{array}$ & Date & $\begin{array}{c}\text { Time } \\
\text { (24- } \\
\text { hour) }\end{array}$ & $\begin{array}{l}\text { Nitro- } \\
\text { gen, } \\
\text { nitrite, } \\
\text { dis- } \\
\text { solved } \\
\text { (mg/L } \\
\text { as } \mathrm{N} \text { ) }\end{array}$ & $\begin{array}{l}\text { Nitro- } \\
\text { gen, } \\
\mathrm{NO}_{2+} \\
\mathrm{NO}_{3}, \\
\text { dis- } \\
\text { solved } \\
\text { (mg/L } \\
\text { as } \mathrm{N} \text { ) }\end{array}$ & $\begin{array}{c}\text { Nitro- } \\
\text { gen, } \\
\text { ammo- } \\
\text { nia, dis- } \\
\text { solved } \\
\text { (mg/L } \\
\text { as } \mathrm{N} \text { ) }\end{array}$ & $\begin{array}{l}\text { Nitro- } \\
\text { gen, } \\
\text { ammo- } \\
\text { nia } \\
\text { plus } \\
\text { organic, } \\
\text { total } \\
\text { (mg/L } \\
\text { as } \mathrm{N} \text { ) }\end{array}$ & $\begin{array}{c}\text { Nitro- } \\
\text { gen, } \\
\text { ammo- } \\
\text { nia } \\
\text { plus } \\
\text { organic, } \\
\text { dis- } \\
\text { solved } \\
\text { (mg/L } \\
\text { as } N \text { ) }\end{array}$ & $\begin{array}{c}\text { Phos- } \\
\text { phorus, } \\
\text { total } \\
\text { (mg/L } \\
\text { as P) }\end{array}$ & $\begin{array}{c}\text { Phos- } \\
\text { phorus, } \\
\text { dis- } \\
\text { solved } \\
\text { (mg/L } \\
\text { as P) }\end{array}$ & $\begin{array}{l}\text { Phos- } \\
\text { phorus, } \\
\text { ortho, } \\
\text { dis- } \\
\text { solved } \\
\text { (mg/L } \\
\text { as P) }\end{array}$ \\
\hline \multirow[t]{4}{*}{1} & $07-27-88$ & 1300 & $<0.01$ & $<0.01$ & 0.06 & 1.3 & 0.40 & 0.16 & 0.05 & 0.04 \\
\hline & $11-15-88$ & $1205^{\circ}$ & $<.01$ & $<.10$ & .05 & 1.3 & .60 & .20 & .03 & .02 \\
\hline & $03-08-89$ & 1000 & .02 & .20 & .03 & .90 & .50 & .16 & .05 & .04 \\
\hline & $06-01-89$ & 1010 & $<.01$ & $<.10$ & .04 & .80 & .60 & .15 & .02 & $<.01$ \\
\hline \multirow[t]{4}{*}{2} & $07-27-88$ & 1150 & $<.01$ & $<.10$ & .04 & .40 & .20 & .10 & .08 & .06 \\
\hline & $11-15-88$ & 1050 & $<.01$ & $<.10$ & .06 & .60 & .50 & .09 & .06 & .04 \\
\hline & 03-08-89 & 1140 & $<.01$ & $<.10$ & .02 & .50 & .50 & .03 & .02 & $<.01$ \\
\hline & $06-01-89$ & 0830 & $<.01$ & $<.10$ & .02 & .70 & .50 & .09 & .04 & .04 \\
\hline \multirow[t]{4}{*}{5} & $07-24-88$ & 1020 & .04 & .80 & .05 & 1.0 & .70 & .32 & .16 & .14 \\
\hline & $11-16-88$ & 0850 & $<.01$ & $<.10$ & .04 & 1.1 & .40 & .17 & .09 & .06 \\
\hline & $03-09-89$ & 0930 & .02 & $<.10$ & .02 & 1.6 & .40 & .28 & .05 & .04 \\
\hline & $06-02-89$ & 0820 & $<.01$ & $<.10$ & .02 & .70 & .60 & .14 & $<.01$ & $<.01$ \\
\hline \multirow[t]{4}{*}{6} & $07-26-88$ & 0730 & .05 & .93 & .15 & 2.0 & .80 & .42 & .19 & .17 \\
\hline & $11-14-88$ & 1200 & .02 & .94 & .20 & 2.3 & 1.5 & .43 & .19 & .07 \\
\hline & $03-08-89$ & 1015 & .10 & 3.1 & 1.2 & 3.6 & 3.6 & .98 & .74 & .69 \\
\hline & $05-31-89$ & 1445 & .10 & .47 & .13 & 1.1 & .80 & .20 & .06 & .06 \\
\hline \multirow[t]{6}{*}{9} & $07-26-88$ & 0945 & .19 & .40 & 2.0 & 2.8 & 2.0 & .51 & .32 & .28 \\
\hline & $07-26-88$ & 2150 & -- & -- & -- & -- & -- & -- & -- & -- \\
\hline & $07-27-88$ & 0550 & - & -- & -- & -- & -- & -- & -- & -- \\
\hline & $11-15-88$ & 0815 & .07 & .49 & 7.2 & 22 & 13 & 4.6 & 4.2 & 3.9 \\
\hline & $03-07-89$ & 1015 & .57 & 3.2 & 3.4 & .60 & .60 & 2.2 & 1.9 & 1.5 \\
\hline & $05-31-89$ & 0930 & .03 & $<.10$ & 6.9 & 11 & 6.4 & -- & - & 2.8 \\
\hline \multirow[t]{5}{*}{10} & $07-26-88$ & 0900 & -- & -- & -- & .90 & 1.8 & .31 & .28 & -- \\
\hline & $07-26-88$ & 1300 & .05 & 2.4 & .07 & .50 & 1.5 & .31 & .28 & .26 \\
\hline & $07-26-88$ & 1700 & .05 & 2.3 & .04 & .30 & 1.0 & .31 & .27 & .25 \\
\hline & $07-26-88$ & 2100 & .05 & 2.2 & .08 & .90 & .90 & .33 & .27 & .23 \\
\hline & $07-27-88$ & 0100 & .04 & 2.1 & .07 & .70 & 1.2 & .32 & .30 & .25 \\
\hline
\end{tabular}


Table 8. Results of ancillary chemical analyses of water samples collected July 1988 through June 1989 in the lower Kansas River Basin-Continued

\begin{tabular}{|c|c|c|c|c|c|c|c|c|c|c|}
\hline $\begin{array}{c}\text { Site } \\
\text { map- } \\
\text { index } \\
\text { number } \\
\text { (fig. 10) }\end{array}$ & Date & $\begin{array}{l}\text { Time } \\
\text { (24- } \\
\text { hour) }\end{array}$ & $\begin{array}{l}\text { Nitro- } \\
\text { gen, } \\
\text { nitrite, } \\
\text { dis- } \\
\text { solved } \\
\text { (mg/L } \\
\text { as N) }\end{array}$ & $\begin{array}{c}\text { Nitro- } \\
\text { gen, } \\
\mathrm{NO}_{2+} \\
\mathrm{NO}_{3}, \\
\text { dis- } \\
\text { solved } \\
\text { (mg/L } \\
\text { as } \mathrm{N})\end{array}$ & $\begin{array}{c}\text { Nitro- } \\
\text { gen, } \\
\text { ammo- } \\
\text { nia, dis- } \\
\text { solved } \\
\text { (mg/L } \\
\text { as } \mathrm{N} \text { ) }\end{array}$ & $\begin{array}{c}\text { Nitro- } \\
\text { gen, } \\
\text { ammo- } \\
\text { nia } \\
\text { plus } \\
\text { organic, } \\
\text { total } \\
\text { (mg/L } \\
\text { as } N \text { ) }\end{array}$ & $\begin{array}{c}\text { Nitro- } \\
\text { gen, } \\
\text { ammo- } \\
\text { nia } \\
\text { plus } \\
\text { organic, } \\
\text { dis- } \\
\text { solved } \\
\text { (mg/L } \\
\text { as } \mathrm{N})\end{array}$ & $\begin{array}{c}\text { Phos- } \\
\text { phorus, } \\
\text { total } \\
\text { (mg/L } \\
\text { as P) }\end{array}$ & $\begin{array}{l}\text { Phos- } \\
\text { phorus, } \\
\text { dis- } \\
\text { solved } \\
\text { (mg/L } \\
\text { as P) }\end{array}$ & $\begin{array}{c}\text { Phos- } \\
\text { phorus, } \\
\text { ortho, } \\
\text { dis- } \\
\text { solved } \\
\text { (mg/L } \\
\text { as P) }\end{array}$ \\
\hline \multirow[t]{5}{*}{10} & $07-27-88$ & 0500 & 0.04 & 2.1 & 0.07 & 1.5 & 1.3 & 0.34 & 0.27 & 0.23 \\
\hline & $07-27-88$ & 0900 & .04 & 2.2 & .09 & .80 & 1.6 & .35 & .27 & .22 \\
\hline & $11-14-88$ & 1345 & $<.01$ & .11 & .06 & 1.4 & 1.4 & .38 & .15 & .05 \\
\hline & $03-07-89$ & 1000 & .05 & 1.6 & 3.2 & 5.4 & 5.2 & 1.0 & .77 & .71 \\
\hline & $05-31-89$ & 1330 & .04 & .17 & .35 & 1.0 & 1.1 & .58 & .52 & .47 \\
\hline \multirow[t]{4}{*}{11} & $07-26-88$ & 0845 & .01 & 2.9 & .05 & 1.4 & .50 & .57 & .39 & .36 \\
\hline & $11-14-88$ & 1050 & .04 & 2.8 & .05 & .50 & .50 & .29 & .26 & .13 \\
\hline & 03-08-89 & 1215 & .10 & 3.1 & .68 & 2.6 & 2. & .65 & .46 & .42 \\
\hline & $05-31-89$ & 1600 & .14 & 2.9 & .19 & .40 & $<.50$ & .37 & .36 & .38 \\
\hline \multirow[t]{9}{*}{12} & $07-26-88$ & 1000 & .05 & 2.3 & .15 & 1.9 & 1.0 & .73 & .47 & .30 \\
\hline & $07-26-88$ & 1005 & .04 & 2.6 & .14 & .60 & .60 & .52 & .32 & .32 \\
\hline & $07-26-88$ & 1400 & .04 & 2.6 & .05 & .70 & .40 & .53 & .34 & .34 \\
\hline & $07-26-88$ & 1800 & .05 & 2.5 & .06 & .60 & .50 & .51 & .33 & .31 \\
\hline & $07-26-88$ & 2200 & -- & -- & -- & -- & -- & -- & -- & -- \\
\hline & $07-27-88$ & 0200 & .40 & 2.5 & .08 & .80 & .40 & .43 & .31 & .34 \\
\hline & $07-27-88$ & 0600 & .04 & 2.4 & .08 & .30 & .20 & .40 & .30 & .26 \\
\hline & $11-14-88$ & 0845 & .03 & 1.9 & .04 & 1.5 & .40 & .33 & .23 & .09 \\
\hline & 03-08-89 & 1130 & .06 & 3.1 & .58 & 2.0 & 2.0 & .58 & .42 & .38 \\
\hline \multirow[t]{7}{*}{16} & $07-26-88$ & 0630 & .16 & .80 & .77 & 8.4 & 8.2 & 4.4 & 3.8 & 3.4 \\
\hline & $07-26-88$ & 1800 & -- & -- & -- & -- & -- & -- & - & - \\
\hline & $07-27-88$ & 1215 & -- & -- & -- & -- & -- & -- & -- & -- \\
\hline & $09-06-88$ & 1145 & -- & -- & -- & -- & -- & -- & -- & -- \\
\hline & $11-15-88$ & 1015 & .01 & .10 & 18 & 21 & 18 & 6.6 & 6.1 & 4.2 \\
\hline & 03-07-89 & 1600 & .21 & .39 & 15 & 18 & -- & 5.9 & 5.4 & 4.6 \\
\hline & $05-30-89$ & 1145 & .05 & .15 & 14 & 14 & -- & -- & - & 4.9 \\
\hline
\end{tabular}


Table 8. Results of ancillary chemical analyses of water samples collected July 1988 through June 1989 in the lower Kansas River Basin-Continued

\begin{tabular}{|c|c|c|c|c|c|c|c|c|c|c|}
\hline $\begin{array}{c}\text { Site } \\
\text { map- } \\
\text { index } \\
\text { number } \\
\text { (fig. 10) }\end{array}$ & Date & $\begin{array}{l}\text { Time } \\
\text { (24- } \\
\text { hour) }\end{array}$ & $\begin{array}{l}\text { Nitro- } \\
\text { gen, } \\
\text { nitrite, } \\
\text { dis- } \\
\text { solved } \\
\text { (mg/L } \\
\text { as N) }\end{array}$ & $\begin{array}{l}\text { Nitro- } \\
\text { gen, } \\
\mathrm{NO}_{2+} \\
\mathrm{NO}_{3}, \\
\text { dis- } \\
\text { solved } \\
\text { (mg/L } \\
\text { as } \mathrm{N} \text { ) }\end{array}$ & $\begin{array}{l}\text { Nitro- } \\
\text { gen, } \\
\text { ammo- } \\
\text { nia, dis- } \\
\text { solved } \\
\text { (mg/L } \\
\text { as } N \text { ) }\end{array}$ & $\begin{array}{c}\text { Nitro- } \\
\text { gen, } \\
\text { ammo- } \\
\text { nia } \\
\text { plus } \\
\text { organic, } \\
\text { total } \\
\text { (mg/L } \\
\text { as } N \text { ) }\end{array}$ & $\begin{array}{c}\text { Nitro- } \\
\text { gen, } \\
\text { ammo- } \\
\text { nia } \\
\text { plus } \\
\text { organic, } \\
\text { dis- } \\
\text { solved } \\
\text { (mg/L } \\
\text { as } \mathrm{N} \text { ) }\end{array}$ & $\begin{array}{l}\text { Phos- } \\
\text { phorus, } \\
\text { total } \\
\text { (mg/L } \\
\text { as P) }\end{array}$ & $\begin{array}{c}\text { Phos- } \\
\text { phorus, } \\
\text { dis- } \\
\text { solved } \\
\text { (mg/L } \\
\text { as P) }\end{array}$ & $\begin{array}{l}\text { Phos- } \\
\text { phorus, } \\
\text { ortho, } \\
\text { dis- } \\
\text { solved } \\
\text { (mg/L } \\
\text { as P) }\end{array}$ \\
\hline \multirow[t]{10}{*}{17} & $07-25-88$ & 1230 & 0.03 & 1.9 & 0.02 & 1.5 & 0.50 & 0.93 & 0.75 & 0.71 \\
\hline & $07-26-88$ & 1300 & .06 & 3.0 & .07 & .80 & .50 & 1.2 & 1.1 & 1.1 \\
\hline & $07-26-88$ & 1700 & .05 & 2.5 & .04 & .70 & .50 & 1.1 & .95 & .92 \\
\hline & $07-26-88$ & 2100 & .07 & 3.0 & .05 & .80 & .80 & 1.2 & 1.0 & .98 \\
\hline & $07-27-88$ & 0100 & .07 & 3.1 & .06 & .60 & .60 & 1.3 & 1.0 & .99 \\
\hline & $07-27-88$ & 0500 & .07 & 2.7 & .07 & .50 & .40 & 1.3 & 1.1 & 1.0 \\
\hline & $07-27-88$ & 0900 & .06 & 2.8 & .06 & .80 & .30 & 1.3 & 1.2 & 1.0 \\
\hline & $07-27-88$ & 1300 & .05 & 2.5 & .06 & .50 & .50 & 1.3 & 1.1 & 1.0 \\
\hline & $11-15-88$ & 1130 & .20 & 6.4 & 4.9 & 6.3 & 5.2 & 5.2 & 4.4 & 4.1 \\
\hline & 03-07-89 & 1300 & .01 & 1.0 & 11 & 11 & 11 & 3.3 & 3.3 & 3.3 \\
\hline \multirow[t]{4}{*}{18} & $07-25-88$ & 1130 & .22 & 1.3 & .29 & 1.1 & 1.0 & .79 & .77 & .73 \\
\hline & $11-15-88$ & 1230 & .18 & 4.9 & .23 & 1.2 & 1.2 & 4.6 & 4.6 & 4.0 \\
\hline & 03-07-89 & 1445 & .20 & 14 & 1.5 & 4.5 & 4.5 & 5.3 & 4.2 & 4.4 \\
\hline & $05-30-89$ & 1445 & 1.0 & 3.3 & 3.3 & 3.8 & 4.3 & 3.7 & 3.1 & 2.8 \\
\hline \multirow[t]{4}{*}{19} & $07-25-88$ & 1200 & -- & -- & -- & 1.7 & 1.2 & .66 & .58 & -- \\
\hline & $11-15-88$ & 1300 & .10 & 2.9 & .05 & 1.0 & 1.1 & 1.1 & 1.0 & .96 \\
\hline & $03-07-89$ & 1245 & .02 & 2.5 & 4.2 & 5.3 & 5.3 & 1.7 & 1.6 & 1.3 \\
\hline & $05-31-89$ & 1100 & .57 & 4.4 & .47 & -- & -- & 1.0 & .86 & .88 \\
\hline \multirow[t]{5}{*}{21} & $07-26-88$ & 1010 & -- & -- & -- & 2.1 & 1.6 & 1.6 & 1.5 & -- \\
\hline & $09-06-88$ & 1440 & -- & -- & -- & -- & -- & -- & -- & -- \\
\hline & $11-14-88$ & 1425 & .10 & 8.5 & $<.01$ & 2.1 & 1.4 & 3.6 & 3.3 & 3.1 \\
\hline & 03-07-89 & 1145 & .18 & 10 & .80 & .80 & .80 & 3.9 & 3.5 & 3.5 \\
\hline & $05-31-89$ & 1245 & .32 & 2.7 & .55 & 1.6 & 1.6 & 3.6 & 3.1 & 2.5 \\
\hline \multirow[t]{5}{*}{23} & $07-25-88$ & 1245 & .02 & 2.3 & .06 & 1.5 & .50 & .67 & .52 & .50 \\
\hline & $08-08-88$ & 1115 & .04 & 2.5 & .04 & 1.3 & -- & .74 & -- & .49 \\
\hline & $08-31-88$ & 1400 & .01 & 1.9 & .04 & 1.2 & -- & .62 & -- & .42 \\
\hline & $10-12-88$ & 1230 & .05 & 1.9 & .12 & 1.0 & -- & .55 & -- & .50 \\
\hline & $11-07-88$ & 1200 & .03 & 2.0 & .04 & .50 & -- & .45 & -- & .35 \\
\hline
\end{tabular}


Table 8. Results of ancillary chemical analyses of water samples collected July 1988 through June 1989 in the lower Kansas River Basin-Continued

\begin{tabular}{|c|c|c|c|c|c|c|c|c|c|c|}
\hline $\begin{array}{c}\text { Site } \\
\text { map- } \\
\text { index } \\
\text { number } \\
\text { (fig. 10) }\end{array}$ & Date & $\begin{array}{c}\text { Time } \\
\text { (24- } \\
\text { hour) }\end{array}$ & $\begin{array}{l}\text { Nitro- } \\
\text { gen, } \\
\text { nitrite, } \\
\text { dis- } \\
\text { solved } \\
\text { (mg/L } \\
\text { as N) }\end{array}$ & $\begin{array}{l}\text { Nitro- } \\
\text { gen, } \\
\mathrm{NO}_{2+} \\
\mathrm{NO}_{3} \text {, } \\
\text { dis- } \\
\text { solved } \\
\text { (mg/L } \\
\text { as } \mathrm{N} \text { ) }\end{array}$ & $\begin{array}{c}\text { Nitro- } \\
\text { gen, } \\
\text { ammo- } \\
\text { nia, dis- } \\
\text { solved } \\
\text { (mg/L } \\
\text { as } \mathrm{N} \text { ) }\end{array}$ & $\begin{array}{c}\text { Nitro- } \\
\text { gen, } \\
\text { ammo- } \\
\text { nia } \\
\text { plus } \\
\text { organic, } \\
\text { total } \\
\text { (mg/L } \\
\text { as } \mathrm{N})\end{array}$ & $\begin{array}{l}\text { Nitro- } \\
\text { gen, } \\
\text { ammo- } \\
\text { nia } \\
\text { plus } \\
\text { organic, } \\
\text { dis- } \\
\text { solved } \\
\text { (mg/L } \\
\text { as } \mathrm{N} \text { ) }\end{array}$ & $\begin{array}{c}\text { Phos- } \\
\text { phorus, } \\
\text { total } \\
\text { (mg/L } \\
\text { as P) }\end{array}$ & $\begin{array}{c}\text { Phos- } \\
\text { phorus, } \\
\text { dis- } \\
\text { solved } \\
\text { (mg/L } \\
\text { as P) }\end{array}$ & $\begin{array}{l}\text { Phos- } \\
\text { phorus, } \\
\text { ortho, } \\
\text { dis- } \\
\text { solved } \\
\text { (mg/L } \\
\text { as P) }\end{array}$ \\
\hline \multirow[t]{9}{*}{23} & $11-15-88$ & 1420 & 0.03 & 2.0 & 0.07 & 0.50 & 0.50 & 0.56 & 0.44 & 0.41 \\
\hline & $12-06-88$ & 1000 & .03 & 2.6 & .06 & .60 & -- & .57 & -- & .48 \\
\hline & 01-03-89 & 1130 & .03 & 2.2 & .53 & 1.1 & -- & .74 & -- & .65 \\
\hline & $02-07-89$ & 1000 & .02 & 2.2 & .61 & 1.0 & -- & .64 & - & .56 \\
\hline & 03-07-89 & 1400 & .03 & 2.8 & 1.1 & 1.6 & -- & .79 & -- & .73 \\
\hline & $03-08-89$ & 1345 & .05 & 2.5 & .85 & 1.7 & .70 & .80 & .62 & .61 \\
\hline & 04-04-89 & 0955 & .02 & 1.6 & .01 & .40 & -- & .47 & -- & .35 \\
\hline & $05-02-89$ & 0830 & .03 & 1.6 & .04 & .30 & -- & .45 & -- & .34 \\
\hline & $05-31-89$ & 1430 & .12 & 2.6 & .11 & 1.1 & .60 & .63 & .54 & .52 \\
\hline \multirow[t]{5}{*}{26} & $07-25-88$ & 1030 & .03 & 1.6 & .09 & 2.0 & .50 & .66 & .47 & .43 \\
\hline & $09-07-88$ & 0840 & -- & -- & -- & -- & -- & -- & - & -- \\
\hline & $11-14-88$ & 1530 & .03 & 1.6 & .21 & .70 & $<.20$ & .54 & .42 & .37 \\
\hline & 03-08-89 & 1500 & .06 & 2.7 & .83 & 2.2 & 2.2 & .74 & .57 & .55 \\
\hline & $05-31-89$ & 1300 & .06 & 2.2 & .04 & 1.7 & .70 & .44 & .35 & .32 \\
\hline \multirow[t]{4}{*}{27} & $07-25-88$ & 1325 & -- & -- & -- & 2.4 & 2.4 & 1.0 & .88 & -- \\
\hline & $11-15-88$ & 1110 & .02 & .93 & .07 & 2.4 & 1.2 & .85 & .59 & .55 \\
\hline & 03-07-89 & 1345 & .05 & 3.2 & .92 & .20 & .20 & 1.6 & 1.5 & 1.3 \\
\hline & $05-30-89$ & 1445 & .12 & .41 & .24 & 1.5 & 1.2 & .74 & .55 & .50 \\
\hline \multirow[t]{4}{*}{28} & $07-25-88$ & 0600 & .06 & 2.0 & .06 & .70 & -- & .50 & .35 & .33 \\
\hline & $11-14-88$ & 1445 & $<.01$ & .64 & .04 & .70 & .20 & .50 & .41 & .39 \\
\hline & $03-07-89$ & 0855 & .04 & 1.6 & .33 & .40 & .30 & .54 & .44 & .37 \\
\hline & $05-31-89$ & 1215 & .03 & .59 & .06 & 1.5 & .80 & .34 & .25 & .22 \\
\hline \multirow[t]{5}{*}{29} & $07-25-88$ & 0630 & .03 & 1.7 & .02 & 1.1 & .60 & .35 & .22 & .22 \\
\hline & $11-14-88$ & 1200 & .02 & 1.9 & .03 & .70 & .70 & .31 & .25 & .22 \\
\hline & 03-07-89 & 1240 & .03 & 2.0 & .27 & .80 & .40 & .39 & .30 & .28 \\
\hline & $05-31-89$ & 1100 & .06 & 1.4 & .04 & .60 & .40 & .40 & .26 & .24 \\
\hline & $07-25-88$ & 1330 & .06 & 1.2 & .06 & 1.6 & .50 & 1.5 & .55 & .50 \\
\hline
\end{tabular}


Table 8. Results of ancillary chemical analyses of water samples collected July 1988 through June 1989 in the lower Kansas River Basin-Continued

\begin{tabular}{|c|c|c|c|c|c|c|c|c|c|c|}
\hline $\begin{array}{c}\text { Site } \\
\text { map- } \\
\text { index } \\
\text { number } \\
\text { (fig. 10) }\end{array}$ & Date & $\begin{array}{l}\text { Time } \\
\text { (24- } \\
\text { hour) }\end{array}$ & $\begin{array}{l}\text { Nitro- } \\
\text { gen, } \\
\text { nitrite, } \\
\text { dis- } \\
\text { solved } \\
\text { (mg/L } \\
\text { as } \mathrm{N} \text { ) }\end{array}$ & $\begin{array}{l}\text { Nitro- } \\
\text { gen, } \\
\mathrm{NO}_{2+} \\
\mathrm{NO}_{3}, \\
\text { dis- } \\
\text { solved } \\
\text { (mg/L } \\
\text { as N) }\end{array}$ & $\begin{array}{l}\text { Nitro- } \\
\text { gen, } \\
\text { ammo- } \\
\text { nia, dis- } \\
\text { solved } \\
\text { (mg/L } \\
\text { as } \mathrm{N} \text { ) }\end{array}$ & $\begin{array}{c}\text { Nitro- } \\
\text { gen, } \\
\text { ammo- } \\
\text { nia } \\
\text { plus } \\
\text { organic, } \\
\text { total } \\
\text { (mg/L } \\
\text { as } \mathrm{N} \text { ) }\end{array}$ & $\begin{array}{c}\text { Nitro- } \\
\text { gen, } \\
\text { ammo- } \\
\text { nia } \\
\text { plus } \\
\text { organic, } \\
\text { dis- } \\
\text { solved } \\
\text { (mg/L } \\
\text { as } \mathrm{N} \text { ) }\end{array}$ & $\begin{array}{c}\text { Phos- } \\
\text { phorus, } \\
\text { total } \\
\text { (mg/L } \\
\text { as P) }\end{array}$ & $\begin{array}{l}\text { Phos- } \\
\text { phorus, } \\
\text { dis- } \\
\text { solved } \\
\text { (mg/L } \\
\text { as P) }\end{array}$ & $\begin{array}{l}\text { Phos- } \\
\text { phorus, } \\
\text { ortho, } \\
\text { dis- } \\
\text { solved } \\
\text { (mg/L } \\
\text { as P) }\end{array}$ \\
\hline \multirow[t]{9}{*}{29} & $07-25-88$ & 1335 & 0.07 & 1.2 & 0.06 & 1.3 & 0.90 & 0.61 & 0.54 & 0.48 \\
\hline & $07-25-88$ & 1730 & .06 & 1.2 & .06 & 1.6 & .90 & .67 & .54 & .49 \\
\hline & $07-25-88$ & 2100 & .05 & 1.2 & .06 & 1.2 & .90 & .63 & .53 & .48 \\
\hline & $07-26-88$ & 0200 & .05 & 1.3 & .11 & 2.4 & 2.6 & .61 & .55 & .48 \\
\hline & $07-26-88$ & 0510 & .05 & 1.2 & .10 & 1.3 & 1.3 & .66 & .53 & .52 \\
\hline & $07-26-88$ & 0915 & .06 & 1.2 & .09 & 1.6 & 1.3 & .76 & .53 & .50 \\
\hline & $11-14-88$ & 1045 & .01 & .23 & .06 & .70 & 1.2 & .68 & .61 & .55 \\
\hline & $03-08-89$ & 0900 & .06 & 1.7 & 1.1 & 3.1 & 3.1 & .61 & .48 & .41 \\
\hline & $05-30-89$ & 1630 & .09 & .34 & .28 & .90 & 1.0 & .92 & .80 & .77 \\
\hline \multirow[t]{14}{*}{31} & $07-25-88$ & 1330 & .03 & 3.0 & .06 & 1.0 & 1.0 & .70 & .61 & .59 \\
\hline & $07-25-88$ & 1700 & .02 & 2.9 & .05 & 1.2 & .70 & .67 & .60 & .59 \\
\hline & $07-25-88$ & 2100 & .03 & 2.9 & .05 & 1.6 & 1.2 & .85 & .60 & .60 \\
\hline & $07-26-88$ & 0115 & .03 & 2.8 & .09 & 1.7 & 1.2 & .77 & .60 & .55 \\
\hline & $07-26-88$ & 0950 & .03 & 2.8 & .05 & .70 & .90 & .75 & .60 & .57 \\
\hline & $07-27-88$ & 0510 & .02 & 2.2 & .06 & .30 & .30 & .68 & .59 & .54 \\
\hline & $11-14-88$ & 0900 & .08 & 2.8 & .06 & 1.2 & .70 & .74 & .60 & .56 \\
\hline & $11-14-88$ & 0905 & .08 & 2.7 & .09 & 1.6 & 1.0 & .68 & .57 & .53 \\
\hline & $11-14-88$ & 1300 & .08 & 2.7 & .04 & 1.1 & .60 & .73 & .58 & .55 \\
\hline & $11-14-88$ & 1305 & .08 & 2.8 & .04 & 1.1 & .50 & .65 & .58 & .56 \\
\hline & $11-14-88$ & 1700 & .08 & 2.7 & .05 & 1.1 & .70 & .74 & .59 & .57 \\
\hline & $11-14-88$ & 1705 & .08 & 2.7 & .05 & .90 & .70 & .64 & .58 & .55 \\
\hline & $11-14-88$ & 2100 & .07 & 2.7 & .06 & 1.3 & 1.1 & .68 & .58 & .55 \\
\hline & $11-14-88$ & 2105 & .07 & 2.7 & .07 & 1.2 & .50 & .72 & .58 & .55 \\
\hline \multirow[t]{5}{*}{32} & $11-15-88$ & 0100 & .07 & 2.8 & .08 & 1.0 & .90 & .72 & .59 & .55 \\
\hline & $11-15-88$ & 0105 & .07 & 2.7 & .08 & 1.4 & .80 & .72 & .58 & .54 \\
\hline & $11-15-88$ & 0500 & .07 & 2.8 & .12 & 1.0 & 1.0 & .64 & .59 & .56 \\
\hline & $11-15-88$ & 0505 & .07 & 3.0 & .13 & 1.1 & .90 & .73 & .60 & .56 \\
\hline & $11-15-88$ & 0900 & .07 & 2.7 & .11 & 1.3 & .50 & .71 & .57 & .55 \\
\hline
\end{tabular}


Table 8. Results of ancillary chemical analyses of water samples collected July 1988 through June 1989 in the lower Kansas River Basin-Continued

\begin{tabular}{|c|c|c|c|c|c|c|c|c|c|c|}
\hline $\begin{array}{c}\text { Site } \\
\text { map- } \\
\text { index } \\
\text { number } \\
\text { (fig. 10) }\end{array}$ & Date & $\begin{array}{c}\text { Time } \\
\text { (24- } \\
\text { hour) }\end{array}$ & $\begin{array}{l}\text { Nitro- } \\
\text { gen, } \\
\text { nitrite, } \\
\text { dis- } \\
\text { solved } \\
\text { (mg/L } \\
\text { as } \mathrm{N})\end{array}$ & $\begin{array}{c}\text { Nitro- } \\
\text { gen, } \\
\mathrm{NO}_{2+} \\
\mathrm{NO}_{3}, \\
\text { dis- } \\
\text { solved } \\
\text { (mg/L } \\
\text { as } \mathrm{N})\end{array}$ & $\begin{array}{l}\text { Nitro- } \\
\text { gen, } \\
\text { ammo- } \\
\text { nia, dis- } \\
\text { solved } \\
\text { (mg/L } \\
\text { as } \mathrm{N} \text { ) }\end{array}$ & $\begin{array}{c}\text { Nitro- } \\
\text { gen, } \\
\text { ammo- } \\
\text { nia } \\
\text { plus } \\
\text { organic, } \\
\text { total } \\
\text { (mg/l } \\
\text { as } \mathrm{N} \text { ) }\end{array}$ & $\begin{array}{c}\text { Nitro- } \\
\text { gen, } \\
\text { ammo- } \\
\text { nia } \\
\text { plus } \\
\text { organic, } \\
\text { dis- } \\
\text { solved } \\
\text { (mg/L } \\
\text { as } \mathrm{N} \text { ) }\end{array}$ & $\begin{array}{c}\text { Phos- } \\
\text { phorus, } \\
\text { total } \\
\text { (mg/L } \\
\text { as P) }\end{array}$ & $\begin{array}{c}\text { Phos- } \\
\text { phorus, } \\
\text { dis- } \\
\text { solved } \\
\text { (mg/L } \\
\text { as P) }\end{array}$ & $\begin{array}{c}\text { Phos- } \\
\text { phorus, } \\
\text { ortho, } \\
\text { dis- } \\
\text { solved } \\
\text { (mg/l } \\
\text { as P) }\end{array}$ \\
\hline \multirow[t]{10}{*}{32} & $11-15-88$ & 0905 & 0.07 & 2.8 & 0.11 & 1.1 & 0.90 & 0.67 & 0.58 & 0.55 \\
\hline & $03-07-89$ & 1000 & .06 & 3.3 & .61 & 1.8 & 1.5 & .76 & .59 & .57 \\
\hline & $03-07-89$ & 1005 & .06 & 3.2 & .61 & 2.1 & 1.1 & .75 & .59 & .56 \\
\hline & $03-07-89$ & 1400 & .06 & 3.2 & .63 & 1.8 & 1.8 & .75 & .60 & .56 \\
\hline & $03-07-89$ & 1405 & .06 & 3.3 & .68 & 2.2 & 2.2 & .70 & .59 & .57 \\
\hline & $03-07-89$ & 1800 & .06 & 3.3 & .64 & 1.9 & 1.2 & .67 & .61 & .57 \\
\hline & 03-07-89 & 1805 & .06 & 3.4 & .65 & 2.4 & 2.1 & .75 & .60 & .54 \\
\hline & 03-07-89 & 2200 & .06 & 3.3 & .67 & 2.1 & 1.9 & .99 & .72 & .70 \\
\hline & $03-07-89$ & 2205 & .06 & 3.2 & .66 & 2.2 & 2.1 & .97 & .79 & .77 \\
\hline & 03-08-89 & 0200 & .06 & 3.1 & .90 & 2.3 & 1.9 & .84 & .67 & .63 \\
\hline & $03-08-89$ & 0205 & .06 & 3.1 & .90 & 2.2 & 1.1 & .84 & .67 & .63 \\
\hline & $03-08-89$ & 0600 & .05 & 3.2 & .81 & 2.2 & 1.9 & .73 & .65 & .62 \\
\hline & 03-08-89 & 0605 & .05 & 3.4 & .79 & 2.1 & 1.8 & .80 & .66 & .61 \\
\hline & 03-08-89 & 1000 & .05 & 3.4 & 1.1 & 2.2 & 1.8 & .82 & .67 & .63 \\
\hline & 03-08-89 & 1005 & .05 & 3.4 & .72 & 2.0 & 2.0 & .81 & .74 & .63 \\
\hline & $05-30-89$ & 1000 & .06 & 1.4 & .03 & 2.2 & .50 & .63 & .36 & .35 \\
\hline & $05-30-89$ & 1005 & .08 & 1.7 & .02 & 3.0 & .60 & .56 & .41 & .39 \\
\hline & $05-30-89$ & 1500 & .07 & 1.5 & .02 & 2.1 & .50 & .53 & .38 & .36 \\
\hline & $05-30-89$ & 1505 & .07 & 1.5 & .06 & 2.7 & 1.1 & .53 & .39 & .36 \\
\hline & $05-30-89$ & 1800 & .09 & 1.8 & .02 & 2.2 & .90 & .52 & .38 & .37 \\
\hline & $05-30-89$ & 1810 & .09 & 1.8 & .03 & 2.5 & 1.2 & .52 & .38 & .37 \\
\hline & $05-30-89$ & 2200 & .09 & 1.9 & .04 & 2.4 & .50 & .53 & .39 & .37 \\
\hline & $05-30-89$ & 2205 & .09 & 1.9 & .03 & 2.9 & .60 & .74 & .41 & .38 \\
\hline & $05-31-89$ & 0200 & .09 & 1.9 & .03 & 1.5 & .50 & .52 & .40 & .38 \\
\hline & $05-31-89$ & 0210 & .09 & 1.9 & .02 & .50 & .50 & .62 & .40 & .37 \\
\hline & $05-31-89$ & 0600 & .07 & 1.4 & .02 & .60 & .30 & .48 & .37 & .35 \\
\hline & $05-31-89$ & 0610 & .09 & 1.9 & .03 & .70 & .70 & .53 & .39 & .38 \\
\hline & $05-31-89$ & 1000 & .09 & 1.8 & .04 & .90 & .80 & .53 & .40 & .38 \\
\hline & $05-31-89$ & 1005 & .09 & 1.9 & .04 & .70 & .90 & .52 & .40 & .38 \\
\hline
\end{tabular}


Table 8. Results of ancillary chemical analyses of water samples collected July 1988 through June 1989 in the lower Kansas River Basin-Continued

\begin{tabular}{|c|c|c|c|c|c|c|c|c|c|c|}
\hline $\begin{array}{c}\text { Site } \\
\text { map- } \\
\text { index } \\
\text { number } \\
\text { (fig. 10) }\end{array}$ & Date & $\begin{array}{l}\text { Time } \\
\text { (24- } \\
\text { hour) }\end{array}$ & $\begin{array}{l}\text { Nitro- } \\
\text { gen, } \\
\text { nitrite, } \\
\text { dis- } \\
\text { solved } \\
\text { (mg/L } \\
\text { as } \mathrm{N} \text { ) }\end{array}$ & $\begin{array}{c}\text { Nitro- } \\
\text { gen, } \\
\mathrm{NO}_{2+} \\
\mathrm{NO}_{3}, \\
\text { dis- } \\
\text { solved } \\
\text { (mg/L } \\
\text { as } \mathrm{N} \text { ) }\end{array}$ & $\begin{array}{l}\text { Nitro- } \\
\text { gen, } \\
\text { ammo- } \\
\text { nia, dis- } \\
\text { solved } \\
\text { (mg/L } \\
\text { as N) }\end{array}$ & $\begin{array}{c}\text { Nitro- } \\
\text { gen, } \\
\text { ammo- } \\
\text { nia } \\
\text { plus } \\
\text { organic, } \\
\text { total } \\
\text { (mg/L } \\
\text { as } \mathrm{N})\end{array}$ & $\begin{array}{c}\text { Nitro- } \\
\text { gen, } \\
\text { ammo- } \\
\text { nia } \\
\text { plus } \\
\text { organic, } \\
\text { dis- } \\
\text { solved } \\
\text { (mg/L } \\
\text { as } \mathrm{N} \text { ) }\end{array}$ & $\begin{array}{c}\text { Phos- } \\
\text { phorus, } \\
\text { total } \\
\text { (mg/L } \\
\text { as P) }\end{array}$ & $\begin{array}{l}\text { Phos- } \\
\text { phorus, } \\
\text { dis- } \\
\text { solved } \\
\text { (mg/L } \\
\text { as P) }\end{array}$ & $\begin{array}{l}\text { Phos- } \\
\text { phorus, } \\
\text { ortho, } \\
\text { dis- } \\
\text { solved } \\
\text { (mg/L } \\
\text { as P) }\end{array}$ \\
\hline \multirow[t]{12}{*}{34} & $07-25-88$ & 0650 & $<0.01$ & 1.6 & 0.06 & 1.6 & 1.1 & 0.33 & 0.21 & 0.20 \\
\hline & $07-26-88$ & 0915 & $<.01$ & 1.4 & .02 & 1.2 & .60 & .27 & .19 & .18 \\
\hline & $07-26-88$ & 1045 & $<.01$ & 1.4 & .06 & 1.3 & .70 & .25 & .20 & .17 \\
\hline & $07-26-88$ & 1445 & $<.01$ & 1.3 & .06 & 1.0 & .50 & .30 & .20 & .16 \\
\hline & $07-26-88$ & 1450 & $<.01$ & 1.3 & .05 & 1.0 & .70 & .28 & .20 & .17 \\
\hline & $07-26-88$ & 1845 & $<.01$ & 1.3 & .05 & 1.2 & .90 & .29 & .20 & .17 \\
\hline & $07-26-88$ & 2230 & $<.01$ & 1.2 & .04 & 1.0 & .80 & .30 & .21 & .19 \\
\hline & $07-27-88$ & 0230 & $<.01$ & 1.3 & .04 & 1.1 & .70 & .33 & .20 & .17 \\
\hline & $07-27-88$ & 0635 & .01 & 1.3 & .07 & 1.8 & .70 & .26 & .20 & .18 \\
\hline & $11-14-88$ & 1020 & $<.01$ & .35 & .11 & .80 & .80 & .22 & .20 & .18 \\
\hline & 03-07-89 & 1520 & .01 & .41 & .09 & .30 & .30 & .19 & .10 & .10 \\
\hline & $05-30-89$ & 1345 & $<.01$ & $<.10$ & .08 & .80 & .60 & .48 & .40 & .37 \\
\hline \multirow[t]{14}{*}{35} & $07-25-88$ & 0540 & .11 & 3.0 & .12 & 2.5 & 2.1 & .74 & .54 & .49 \\
\hline & 08-09-88 & 0915 & .09 & .75 & .44 & 1.2 & -- & .56 & -- & .41 \\
\hline & $08-30-88$ & 1400 & .11 & 2.0 & .23 & 2.5 & - & .76 & -- & .47 \\
\hline & $10-18-88$ & 1000 & .06 & 2.6 & .05 & .60 & -- & .59 & -- & .48 \\
\hline & $11-08-88$ & 1200 & .03 & 1.8 & .09 & 1.6 & -- & .70 & .50 & .44 \\
\hline & $11-14-88$ & 1145 & .03 & 1.8 & .09 & 1.5 & .60 & .55 & .43 & .38 \\
\hline & $12-06-88$ & 0900 & .04 & 2.0 & .01 & 1.5 & -- & .52 & - & .34 \\
\hline & 01-04-89 & 1100 & .03 & 2.7 & .03 & 1.8 & -- & .53 & .43 & .38 \\
\hline & $01-31-89$ & 1100 & .05 & 3.3 & .02 & 2.3 & -- & .66 & - & .45 \\
\hline & 03-07-89 & 1435 & .05 & 2.9 & .57 & .50 & .20 & .68 & .56 & .53 \\
\hline & $03-28-89$ & 1130 & .08 & 3.4 & .47 & -- & -- & .65 & .58 & .57 \\
\hline & $04-19-89$ & 1130 & .04 & .53 & .06 & .40 & -- & .24 & -- & .10 \\
\hline & $05-24-89$ & 0940 & $<.01$ & $<.10$ & .02 & 2.0 & - & .47 & .23 & .21 \\
\hline & $05-30-89$ & 1245 & .06 & .27 & .41 & 1.3 & .90 & .57 & .38 & .34 \\
\hline
\end{tabular}


Table 8. Results of ancillary chemical analyses of water samples collected July 1988 through June 1989 in the lower Kansas River Basin-Continued

\begin{tabular}{|c|c|c|c|c|c|c|c|c|c|c|}
\hline $\begin{array}{c}\text { Site } \\
\text { map- } \\
\text { index } \\
\text { number } \\
\text { (fig. 10) }\end{array}$ & Date & $\begin{array}{c}\text { Time } \\
\text { (24- } \\
\text { hour) }\end{array}$ & $\begin{array}{c}\text { Nitro- } \\
\text { gen, } \\
\text { nitrite, } \\
\text { dis- } \\
\text { solved } \\
\text { (mg/L } \\
\text { as N) }\end{array}$ & $\begin{array}{l}\text { Nitro- } \\
\text { gen, } \\
\mathrm{NO}_{2+} \\
\mathrm{NO}_{3} \text {, } \\
\text { dis- } \\
\text { solved } \\
\text { (mg/L } \\
\text { as } \mathrm{N} \text { ) }\end{array}$ & $\begin{array}{l}\text { Nitro- } \\
\text { gen, } \\
\text { ammo- } \\
\text { nia, dis- } \\
\text { solved } \\
\text { (mg/L } \\
\text { as N) }\end{array}$ & $\begin{array}{l}\text { Nitro- } \\
\text { gen, } \\
\text { ammo- } \\
\text { nia } \\
\text { plus } \\
\text { organic, } \\
\text { total } \\
\text { (mg/L } \\
\text { as N) }\end{array}$ & $\begin{array}{c}\text { Nitro- } \\
\text { gen, } \\
\text { ammo- } \\
\text { nia } \\
\text { plus } \\
\text { organic, } \\
\text { dis- } \\
\text { solved } \\
\text { (mg/L } \\
\text { as } \mathrm{N} \text { ) }\end{array}$ & $\begin{array}{c}\text { Phos- } \\
\text { phorus, } \\
\text { total } \\
\text { (mg/L } \\
\text { as P) }\end{array}$ & $\begin{array}{c}\text { Phos- } \\
\text { phorus, } \\
\text { dis- } \\
\text { solved } \\
\text { (mg/L } \\
\text { as P) }\end{array}$ & $\begin{array}{l}\text { Phos- } \\
\text { phorus, } \\
\text { ortho, } \\
\text { dis- } \\
\text { solved } \\
\text { (mg/L } \\
\text { as P) }\end{array}$ \\
\hline \multirow[t]{4}{*}{36} & $07-25-88$ & 1230 & 0.06 & 2.9 & 0.02 & 0.60 & 0.50 & 0.62 & 0.57 & 0.53 \\
\hline & $11-16-88$ & 1050 & .03 & 1.6 & .06 & 1.4 & .50 & .60 & .38 & .35 \\
\hline & 03-09-89 & 0940 & .05 & 2.9 & .47 & 1.6 & 1.3 & .64 & .51 & .48 \\
\hline & $05-31-89$ & 1240 & $<.01$ & $<.10$ & .02 & 1.0 & .70 & .42 & .21 & .21 \\
\hline \multirow[t]{4}{*}{37} & $07-26-88$ & 0515 & .05 & 1.4 & .07 & 1.9 & .60 & .64 & .37 & .36 \\
\hline & $11-15-88$ & 1100 & .02 & .66 & .04 & .30 & .20 & .29 & .24 & .22 \\
\hline & 03-08-89 & 0800 & .03 & 1.1 & .17 & .70 & .70 & .30 & .27 & .28 \\
\hline & $05-30-89$ & 1200 & .03 & .32 & .07 & .40 & .50 & .31 & .29 & .27 \\
\hline \multirow[t]{4}{*}{38} & $07-26-88$ & 0810 & .02 & 1.4 & .02 & .80 & .40 & .62 & .40 & .39 \\
\hline & $11-15-88$ & 0940 & .03 & .78 & .01 & .30 & .20 & .22 & .20 & .19 \\
\hline & $03-08-89$ & 0920 & .02 & 1.4 & .06 & 1.0 & .80 & .34 & .24 & .23 \\
\hline & $05-30-89$ & 1320 & .03 & .70 & .07 & .50 & .40 & .30 & .27 & .26 \\
\hline \multirow[t]{4}{*}{39} & $07-26-88$ & 0630 & .03 & 1.5 & .04 & 2.0 & .90 & .61 & .40 & .39 \\
\hline & $11-15-88$ & 0815 & .02 & .48 & .02 & .40 & .20 & .22 & .19 & .18 \\
\hline & 03-08-89 & 1100 & .02 & 1.3 & .07 & .60 & .40 & .24 & .20 & .19 \\
\hline & $05-31-89$ & 1030 & .03 & .76 & .09 & .30 & .50 & .39 & .35 & .34 \\
\hline \multirow[t]{4}{*}{40} & $07-26-88$ & 0630 & .04 & .82 & .02 & 3.0 & 1.2 & .59 & .30 & .29 \\
\hline & $11-14-88$ & 1655 & $<.01$ & .70 & .03 & .70 & .30 & .25 & .24 & .22 \\
\hline & $03-08-89$ & 1345 & .02 & 1.6 & .09 & 1.3 & 1.1 & .31 & .23 & .22 \\
\hline & $05-31-89$ & 1200 & .02 & 1.2 & .08 & .30 & $<.20$ & .46 & .40 & .38 \\
\hline 41 & $07-26-88$ & 0600 & .06 & 2.8 & .09 & 1.6 & .90 & .46 & .35 & .30 \\
\hline \multirow[t]{4}{*}{42} & $07-26-88$ & 0545 & .02 & 1.7 & .03 & .70 & .60 & .54 & .47 & .45 \\
\hline & $11-14-88$ & 1730 & $<.01$ & 1.5 & .01 & .30 & .30 & .24 & .23 & .22 \\
\hline & 03-08-89 & 1250 & .01 & 1.4 & .04 & .60 & .60 & .27 & .24 & .25 \\
\hline & $05-31-89$ & 1130 & .02 & 1.3 & .07 & .30 & .40 & .25 & .23 & .22 \\
\hline
\end{tabular}


Table 8. Results of ancillary chemical analyses of water samples collected July 1988 through June 1989 in the lower Kansas River Basin-Continued

\begin{tabular}{|c|c|c|c|c|c|c|c|c|c|c|}
\hline $\begin{array}{c}\text { Site } \\
\text { map- } \\
\text { index } \\
\text { number } \\
\text { (fig. 10) }\end{array}$ & Date & $\begin{array}{l}\text { Time } \\
\text { (24- } \\
\text { hour) }\end{array}$ & $\begin{array}{l}\text { Nitro- } \\
\text { gen, } \\
\text { nitrite, } \\
\text { dis- } \\
\text { solved } \\
\text { (mg/L } \\
\text { as N) }\end{array}$ & $\begin{array}{l}\text { Nitro- } \\
\text { gen, } \\
\mathrm{NO}_{2} \\
\mathrm{NO}_{3}, \\
\text { dis- } \\
\text { solved } \\
\text { (mg/L } \\
\text { as } \mathrm{N} \text { ) }\end{array}$ & $\begin{array}{l}\text { Nitro- } \\
\text { gen, } \\
\text { ammo- } \\
\text { nia, dis- } \\
\text { solved } \\
\text { (mg/L } \\
\text { as } \mathbf{N} \text { ) }\end{array}$ & $\begin{array}{c}\text { Nitro- } \\
\text { gen, } \\
\text { ammo- } \\
\text { nia } \\
\text { plus } \\
\text { organic, } \\
\text { total } \\
\text { (mg/L } \\
\text { as N) }\end{array}$ & $\begin{array}{c}\text { Nitro- } \\
\text { gen, } \\
\text { ammo- } \\
\text { nia } \\
\text { plus } \\
\text { organic, } \\
\text { dis- } \\
\text { solved } \\
\text { (mg/L } \\
\text { as } \mathrm{N} \text { ) }\end{array}$ & $\begin{array}{l}\text { Phos- } \\
\text { phorus, } \\
\text { total } \\
\text { (mg/L } \\
\text { as P) }\end{array}$ & $\begin{array}{c}\text { Phos- } \\
\text { phorus, } \\
\text { dis- } \\
\text { solved } \\
\text { (mg/L } \\
\text { as P) }\end{array}$ & $\begin{array}{l}\text { Phos- } \\
\text { phorus, } \\
\text { ortho, } \\
\text { dis- } \\
\text { solved } \\
\text { (mg/L } \\
\text { as P) }\end{array}$ \\
\hline \multirow[t]{9}{*}{44} & $07-25-88$ & 0845 & 0.02 & 0.19 & $<0.01$ & 1.6 & 1.0 & 0.52 & 0.34 & 0.33 \\
\hline & $07-26-88$ & 1320 & .04 & .56 & .04 & 2.4 & .50 & .51 & .33 & .29 \\
\hline & $07-26-88$ & 1710 & .02 & .32 & .07 & 1.9 & .70 & .51 & .27 & .27 \\
\hline & $07-26-88$ & 2150 & .03 & .41 & .07 & 1.3 & .90 & .31 & .30 & .26 \\
\hline & $07-27-88$ & 0120 & .04 & .53 & .07 & 1.6 & .40 & .54 & .31 & .30 \\
\hline & $07-27-88$ & 0545 & .02 & .28 & .03 & 1.8 & .20 & .60 & .28 & .22 \\
\hline & $11-14-88$ & 1555 & $<.01$ & 1.0 & .05 & .80 & .50 & .37 & .30 & .27 \\
\hline & $03-08-89$ & 1225 & .02 & 1.6 & .27 & .70 & .60 & .32 & .27 & .27 \\
\hline & $05-31-89$ & 1300 & .04 & 1.4 & .32 & .50 & .70 & .45 & .42 & .40 \\
\hline \multirow[t]{9}{*}{46} & $07-25-88$ & 0615 & .05 & 2.7 & .10 & 1.8 & 1.7 & .29 & .17 & .16 \\
\hline & $07-26-88$ & 1355 & .03 & 2.6 & .03 & 1.2 & .50 & .34 & .19 & .16 \\
\hline & $07-26-88$ & 1750 & .03 & 2.6 & .06 & .60 & .60 & .23 & .18 & .17 \\
\hline & $07-26-88$ & 2110 & .03 & 2.5 & .02 & 1.7 & .60 & .54 & .16 & .14 \\
\hline & $07-27-88$ & 0100 & .02 & 2.6 & .05 & .80 & .70 & .21 & .17 & .17 \\
\hline & $07-27-88$ & 0510 & .02 & 2.5 & .04 & 2.7 & .20 & .34 & .19 & .15 \\
\hline & $11-14-88$ & 1515 & $<.01$ & .98 & .03 & .60 & .30 & .13 & .09 & .08 \\
\hline & $03-08-89$ & 1200 & .02 & 1.6 & .09 & .80 & .30 & .11 & .07 & .07 \\
\hline & $05-31-89$ & 1330 & .13 & 1.6 & .16 & .70 & .80 & .20 & .15 & .14 \\
\hline \multirow[t]{6}{*}{47} & $07-25-88$ & 1150 & .02 & .42 & .04 & 2.1 & 1.2 & .61 & .36 & .32 \\
\hline & $07-27-88$ & 0920 & $<.01$ & $<.10$ & .04 & 1.6 & -- & .59 & -- & .20 \\
\hline & $08-09-88$ & 1135 & .04 & .37 & .13 & 1.6 & -- & .31 & -- & .21 \\
\hline & $08-30-88$ & 1100 & .02 & .61 & .02 & 1.1 & -- & .45 & -- & .24 \\
\hline & $10-18-88$ & 1300 & .02 & 1.3 & .01 & .40 & -- & .39 & -- & .33 \\
\hline & $11-09-88$ & 1200 & .02 & .97 & .03 & .40 & -- & .30 & - & .22 \\
\hline
\end{tabular}


Table 8. Results of ancillary chemical analyses of water samples collected July 1988 through June 1989 in the lower Kansas River Basin-Continued

\begin{tabular}{|c|c|c|c|c|c|c|c|c|c|c|}
\hline $\begin{array}{c}\text { Site } \\
\text { map- } \\
\text { index } \\
\text { number } \\
\text { (fig. 10) }\end{array}$ & Date & $\begin{array}{c}\text { Time } \\
\text { (24- } \\
\text { hour) }\end{array}$ & $\begin{array}{l}\text { Nitro- } \\
\text { gen, } \\
\text { nitrite, } \\
\text { dis- } \\
\text { solved } \\
\text { (mg/L } \\
\text { as N) }\end{array}$ & $\begin{array}{l}\text { Nitro- } \\
\text { gen, } \\
\mathrm{NO}_{2}+ \\
\mathrm{NO}_{3}, \\
\text { dis- } \\
\text { solved } \\
\text { (mg/h } \\
\text { as } \mathrm{N} \text { ) }\end{array}$ & $\begin{array}{l}\text { Nitro- } \\
\text { gen, } \\
\text { ammo- } \\
\text { nia, dis- } \\
\text { solved } \\
\text { (mg/L } \\
\text { as N) }\end{array}$ & $\begin{array}{c}\text { Nitro- } \\
\text { gen, } \\
\text { ammo- } \\
\text { nia } \\
\text { plus } \\
\text { organic, } \\
\text { total } \\
\text { (mg/L } \\
\text { as N) }\end{array}$ & $\begin{array}{c}\text { Nitro- } \\
\text { gen, } \\
\text { ammo- } \\
\text { nia } \\
\text { plus } \\
\text { organic, } \\
\text { dis- } \\
\text { solved } \\
\text { (mg/L } \\
\text { as } \mathrm{N} \text { ) }\end{array}$ & $\begin{array}{c}\text { Phos- } \\
\text { phorus, } \\
\text { total } \\
\text { (mg/L } \\
\text { as P) }\end{array}$ & $\begin{array}{c}\text { Phos- } \\
\text { phorus, } \\
\text { dis- } \\
\text { solved } \\
\text { (mglL } \\
\text { as P) }\end{array}$ & $\begin{array}{l}\text { Phos- } \\
\text { phorus, } \\
\text { ortho, } \\
\text { dis- } \\
\text { solved } \\
\text { (mg/L } \\
\text { as P) }\end{array}$ \\
\hline \multirow[t]{9}{*}{47} & $11-14-88$ & 1350 & 0.01 & 1.0 & 0.02 & 0.30 & 0.30 & 0.24 & 0.23 & 0.21 \\
\hline & $12-06-88$ & 1300 & .02 & 1.5 & $<.01$ & .30 & -- & .25 & -- & .20 \\
\hline & $01-10-89$ & 1050 & .03 & 2.0 & .12 & .50 & -- & .23 & - & .19 \\
\hline & $02-07-89$ & 1115 & .02 & 2.4 & .18 & .60 & -- & .27 & -- & .21 \\
\hline & $03-07-89$ & 1100 & .20 & 1.8 & .18 & .60 & -- & .27 & -- & .25 \\
\hline & $03-08-89$ & 1040 & .02 & 1.6 & .20 & .40 & .20 & .30 & .24 & .23 \\
\hline & $04-05-89$ & 0955 & .03 & .78 & .03 & .20 & - & .25 & - & .21 \\
\hline & $05-01-89$ & 1200 & .02 & .71 & .05 & $<.20$ & - & .23 & -- & .17 \\
\hline & $05-31-89$ & 1420 & .04 & 1.4 & .06 & .40 & .60 & .41 & .36 & .34 \\
\hline \multirow[t]{4}{*}{48} & $07-26-88$ & 1215 & .01 & .15 & .07 & .60 & .60 & .13 & .07 & .06 \\
\hline & $11-16-88$ & 1400 & $<.01$ & $<.10$ & .11 & 1.1 & .80 & .15 & .08 & .06 \\
\hline & 03-09-89 & 0830 & $<.01$ & .64 & .04 & 1.0 & .80 & .06 & .03 & .02 \\
\hline & 05-31-89 & 1415 & $<.01$ & $<.10$ & .03 & .30 & .60 & .15 & .08 & .05 \\
\hline \multirow[t]{11}{*}{49} & $07-25-88$ & 1400 & .02 & 1.0 & .03 & .60 & .50 & .42 & .32 & .30 \\
\hline & $11-16-88$ & 1130 & .02 & .88 & .23 & .80 & .80 & .40 & .31 & .27 \\
\hline & $11-16-88$ & 1135 & .02 & .91 & .23 & 1.1 & .80 & .40 & .31 & .28 \\
\hline & 03-08-89 & 1630 & .02 & 1.7 & .12 & .90 & .90 & .25 & .18 & .15 \\
\hline & 03-08-89 & 1635 & .02 & 1.7 & .15 & .90 & .50 & .26 & .18 & .16 \\
\hline & 03-08-89 & 1715 & .02 & 1.7 & .13 & 1.2 & .80 & .28 & .18 & .17 \\
\hline & 03-08-89 & 1720 & .02 & 1.8 & .14 & 1.0 & 1.0 & .27 & .19 & .16 \\
\hline & $05-31-89$ & 1540 & .03 & .63 & .03 & $<.20$ & $<.20$ & .32 & .27 & .25 \\
\hline & $05-31-89$ & 1545 & .03 & .63 & .02 & $<.20$ & .30 & .33 & .26 & .25 \\
\hline & $05-31-89$ & 1550 & .03 & .63 & .03 & .60 & .40 & .31 & .25 & .25 \\
\hline & $05-31-89$ & 1555 & .03 & .63 & .03 & 1.1 & .50 & .31 & .25 & .25 \\
\hline
\end{tabular}


Table 8. Results of ancillary chemical analyses of water samples collected July 1988 through June 1989 in the lower Kansas River Basin-Continued

\begin{tabular}{|c|c|c|c|c|c|c|c|c|c|c|}
\hline $\begin{array}{c}\text { Site } \\
\text { map- } \\
\text { index } \\
\text { number } \\
\text { (fig. 10) }\end{array}$ & Date & $\begin{array}{c}\text { Time } \\
\text { (24- } \\
\text { hour) }\end{array}$ & $\begin{array}{c}\text { Nitro- } \\
\text { gen, } \\
\text { nitrite, } \\
\text { dis- } \\
\text { solved } \\
\text { (mg/L } \\
\text { as N) }\end{array}$ & $\begin{array}{c}\text { Nitro- } \\
\text { gen, } \\
\mathrm{NO}_{2+} \\
\mathrm{NO}_{3}, \\
\text { dis- } \\
\text { solved } \\
\text { (mg/L } \\
\text { as } \mathrm{N} \text { ) }\end{array}$ & $\begin{array}{l}\text { Nitro- } \\
\text { gen, } \\
\text { ammo- } \\
\text { nia, dis- } \\
\text { solved } \\
\text { (mg/L } \\
\text { as N) }\end{array}$ & $\begin{array}{c}\text { Nitro- } \\
\text { gen, } \\
\text { ammo- } \\
\text { nia } \\
\text { plus } \\
\text { organic, } \\
\text { total } \\
\text { (mg/L } \\
\text { as N) }\end{array}$ & $\begin{array}{c}\text { Nitro- } \\
\text { gen, } \\
\text { ammo- } \\
\text { nia } \\
\text { plus } \\
\text { organic, } \\
\text { dis- } \\
\text { solved } \\
\text { (mg/L } \\
\text { as } \mathrm{N} \text { ) }\end{array}$ & $\begin{array}{c}\text { Phos- } \\
\text { phorus, } \\
\text { total } \\
\text { (mg/L } \\
\text { as P) }\end{array}$ & $\begin{array}{c}\text { Phos- } \\
\text { phorus, } \\
\text { dis- } \\
\text { solved } \\
\text { (mg/L } \\
\text { as P) }\end{array}$ & $\begin{array}{c}\text { Phos- } \\
\text { phorus, } \\
\text { ortho, } \\
\text { dis- } \\
\text { solved } \\
\text { (mg/l } \\
\text { as P) }\end{array}$ \\
\hline \multirow[t]{6}{*}{50} & $07-24-88$ & 1320 & 0.04 & 0.48 & 0.04 & 1.2 & 5.0 & 0.28 & 0.15 & 0.14 \\
\hline & $11-16-88$ & 1340 & $<.01$ & $<.10$ & .03 & .90 & .60 & .27 & .19 & .13 \\
\hline & $11-16-88$ & 1350 & $<.01$ & .17 & .05 & .80 & .40 & .32 & .20 & .18 \\
\hline & $11-16-88$ & 1355 & -- & - & -- & - & -- & -- & -- & - \\
\hline & 03-09-89 & 1130 & .01 & .35 & .04 & .50 & .40 & .11 & .06 & .06 \\
\hline & $05-31-89$ & 1055 & .02 & .11 & .03 & .60 & .70 & .22 & .18 & .15 \\
\hline \multirow[t]{4}{*}{51} & $07-24-88$ & 1445 & $<.01$ & $<.10$ & .04 & 1.2 & .80 & .16 & .06 & .04 \\
\hline & $11-16-88$ & 0940 & $<.01$ & $<.10$ & .10 & .70 & .50 & .12 & .06 & .05 \\
\hline & $03-09-89$ & 1115 & $<.01$ & $<.10$ & .04 & .70 & .70 & .06 & .01 & $<.01$ \\
\hline & $05-31-89$ & 1730 & .02 & $<.10$ & .12 & 1.0 & .60 & .10 & .08 & .06 \\
\hline \multirow[t]{4}{*}{52} & $07-24-88$ & 1045 & .04 & .70 & .07 & .60 & .70 & .08 & .07 & .06 \\
\hline & $11-15-88$ & 1445 & $<.01$ & .83 & .06 & .60 & .60 & .12 & .09 & .07 \\
\hline & $03-08-89$ & 1430 & .02 & .77 & .51 & 1.4 & 1.1 & .21 & .13 & .11 \\
\hline & $06-02-89$ & 1030 & .03 & .80 & .06 & .60 & .50 & .09 & .06 & .06 \\
\hline \multirow[t]{4}{*}{55} & $07-27-88$ & 0930 & .01 & $<.10$ & .01 & 1.0 & .80 & .19 & .10 & .08 \\
\hline & $11-15-88$ & 1330 & $<.01$ & .20 & .06 & 1.1 & .90 & .17 & .05 & .04 \\
\hline & 03-08-89 & 1345 & $<.01$ & $<.10$ & .47 & .90 & .70 & .24 & .20 & .18 \\
\hline & $05-31-89$ & 0900 & $<.01$ & $<.10$ & .04 & .50 & .60 & .07 & .03 & .02 \\
\hline \multirow[t]{4}{*}{56} & $07-27-88$ & 1120 & $<.01$ & $<.10$ & .05 & 1.2 & .30 & .07 & .03 & .03 \\
\hline & $11-15-88$ & 1115 & $<.01$ & .11 & .05 & .60 & .60 & .12 & .04 & .03 \\
\hline & 03-08-89 & 1100 & .01 & $<.10$ & .03 & .30 & .30 & .04 & .01 & .01 \\
\hline & 06-01-89 & 1450 & $<.01$ & $<.10$ & .02 & .70 & .50 & .06 & .02 & .01 \\
\hline \multirow[t]{3}{*}{57} & $07-29-88$ & 0815 & $<.01$ & .57 & .02 & .60 & .60 & .12 & .12 & .04 \\
\hline & 03-08-89 & 1645 & .01 & .44 & .04 & .40 & .20 & .05 & .03 & .02 \\
\hline & 06-01-89 & 1645 & .02 & .71 & .07 & .60 & .60 & .11 & .08 & .07 \\
\hline
\end{tabular}


Table 8. Results of ancillary chemical analyses of water samples collected July 1988 through June 1989 in the lower Kansas River Basin-Continued

\begin{tabular}{|c|c|c|c|c|c|c|c|c|c|c|}
\hline $\begin{array}{c}\text { Site } \\
\text { map- } \\
\text { index } \\
\text { number } \\
\text { (fig. 10) }\end{array}$ & Date & $\begin{array}{c}\text { Time } \\
\text { (24- } \\
\text { hour) }\end{array}$ & $\begin{array}{c}\text { Nitro- } \\
\text { gen, } \\
\text { nitrite, } \\
\text { dis- } \\
\text { solved } \\
\text { (mg/L } \\
\text { as N) }\end{array}$ & $\begin{array}{c}\text { Nitro- } \\
\text { gen, } \\
\mathrm{NO}_{2+} \\
\mathrm{NO}_{3}, \\
\text { dis- } \\
\text { solved } \\
\text { (mg/L } \\
\text { as } \mathrm{N})\end{array}$ & $\begin{array}{l}\text { Nitro- } \\
\text { gen, } \\
\text { ammo- } \\
\text { nia, dis- } \\
\text { solved } \\
\text { (mg/L } \\
\text { as } \mathrm{N} \text { ) }\end{array}$ & $\begin{array}{c}\text { Nitro- } \\
\text { gen, } \\
\text { ammo- } \\
\text { nia } \\
\text { plus } \\
\text { organic, } \\
\text { total } \\
\text { (mg/L } \\
\text { as } \mathrm{N} \text { ) }\end{array}$ & $\begin{array}{l}\text { Nitro- } \\
\text { gen, } \\
\text { ammo- } \\
\text { nia } \\
\text { plus } \\
\text { organic, } \\
\text { dis- } \\
\text { solved } \\
\text { (mg/L } \\
\text { as } \mathrm{N} \text { ) }\end{array}$ & $\begin{array}{c}\text { Phos- } \\
\text { phorus, } \\
\text { total } \\
\text { (mg/L } \\
\text { as P) }\end{array}$ & $\begin{array}{c}\text { Phos- } \\
\text { phorus, } \\
\text { dis- } \\
\text { solved } \\
\text { (mg/L } \\
\text { as P) }\end{array}$ & $\begin{array}{l}\text { Phos- } \\
\text { phorus, } \\
\text { ortho, } \\
\text { dis- } \\
\text { solved } \\
\text { (mg/L } \\
\text { as P) }\end{array}$ \\
\hline \multirow[t]{4}{*}{58} & $07-27-88$ & 0910 & 0.03 & 0.41 & 0.08 & 0.30 & $<0.20$ & 0.14 & 0.05 & 0.04 \\
\hline & $11-15-88$ & 1445 & $<.01$ & .30 & .04 & .90 & .50 & .15 & .09 & .08 \\
\hline & $03-10-89$ & 1110 & .02 & .44 & .04 & 1.3 & .90 & .21 & .07 & .06 \\
\hline & $05-30-89$ & 1845 & .10 & .98 & .16 & .80 & .90 & .30 & .14 & .13 \\
\hline \multirow[t]{4}{*}{59} & $07-27-88$ & 1010 & .01 & 1.2 & .06 & .50 & .50 & .06 & .04 & .02 \\
\hline & $11-15-88$ & 1100 & $<.01$ & $<.10$ & .03 & .60 & .40 & .06 & .02 & .02 \\
\hline & $03-08-89$ & 1310 & $<.01$ & $<.10$ & .07 & .30 & .80 & .06 & .03 & .02 \\
\hline & $06-01-89$ & 1145 & $<.01$ & $<.10$ & .05 & .50 & .40 & .04 & .02 & .01 \\
\hline \multirow[t]{4}{*}{60} & $07-29-88$ & 0535 & .02 & .15 & .07 & .80 & .60 & .11 & .04 & .03 \\
\hline & $11-15-88$ & 1240 & $<.01$ & $<.10$ & .06 & 1.5 & .70 & .20 & .09 & .06 \\
\hline & $03-09-89$ & 1230 & $<.01$ & $<.10$ & .02 & .60 & .30 & .03 & .01 & .02 \\
\hline & $06-01-89$ & 1310 & $<.01$ & .14 & .11 & .70 & .50 & .07 & .03 & .04 \\
\hline \multirow[t]{5}{*}{61} & $07-28-88$ & 1745 & $<.01$ & $<.10$ & .02 & 1.3 & .30 & .07 & .02 & $<.01$ \\
\hline & $07-29-88$ & 0415 & $<.01$ & $<.10$ & .03 & .90 & .70 & .12 & .01 & $<.01$ \\
\hline & $11-15-88$ & 1030 & $<.01$ & .26 & .03 & .90 & $<.20$ & .13 & .09 & .08 \\
\hline & 03-08-89 & 1030 & .02 & .67 & .20 & 1.2 & 1.2 & .32 & .24 & .20 \\
\hline & $05-31-89$ & 1710 & .08 & .92 & .07 & .40 & .60 & .28 & .13 & .13 \\
\hline \multirow[t]{6}{*}{62} & $07-28-88$ & 1815 & $<.01$ & $<.10$ & .02 & .40 & .30 & .16 & .02 & .01 \\
\hline & $07-29-88$ & 0530 & $<.01$ & $<.10$ & .02 & .80 & .80 & .15 & .02 & $<.01$ \\
\hline & $07-29-88$ & 0535 & $<.01$ & .25 & .02 & .70 & .70 & .10 & .02 & $<.01$ \\
\hline & $11-15-88$ & 0755 & $<.01$ & .29 & .03 & .70 & .40 & .13 & .09 & .08 \\
\hline & 03-07-89 & 1510 & .02 & .39 & .12 & 2.0 & 1.3 & .19 & .09 & .07 \\
\hline & $06-01-89$ & 1815 & .05 & .79 & .05 & .50 & .60 & .15 & .13 & .11 \\
\hline \multirow[t]{4}{*}{63} & $07-29-88$ & 0910 & $<.01$ & $<.10$ & .02 & .90 & .30 & .07 & .02 & $<.01$ \\
\hline & $11-14-88$ & 1000 & $<.01$ & $<.10$ & .08 & .30 & .30 & .06 & .01 & .02 \\
\hline & 03-07-89 & 1010 & $<.01$ & $<.10$ & .04 & 1.2 & .60 & .02 & .01 & .01 \\
\hline & $06-01-89$ & 1300 & $<.01$ & $<.10$ & .04 & .70 & .60 & .06 & .03 & .02 \\
\hline
\end{tabular}


Table 8. Results of ancillary chemical analyses of water samples collected July 1988 through June 1989 in the lower Kansas River Basin-Continued

\begin{tabular}{|c|c|c|c|c|c|c|c|c|c|c|}
\hline $\begin{array}{c}\text { Site } \\
\text { map- } \\
\text { index } \\
\text { number } \\
\text { (fig. 10) }\end{array}$ & Date & $\begin{array}{l}\text { Time } \\
\text { (24- } \\
\text { hour) }\end{array}$ & $\begin{array}{c}\text { Nitro- } \\
\text { gen, } \\
\text { nitrite, } \\
\text { dis- } \\
\text { solved } \\
\text { (mg/L } \\
\text { as } \mathrm{N})\end{array}$ & $\begin{array}{l}\text { Nitro- } \\
\text { gen, } \\
\mathrm{NO}_{2+} \\
\mathrm{NO}_{3}, \\
\text { dis- } \\
\text { solved } \\
\text { (mg/L } \\
\text { as } \mathrm{N})\end{array}$ & $\begin{array}{l}\text { Nitro- } \\
\text { gen, } \\
\text { ammo- } \\
\text { nia, dis- } \\
\text { solved } \\
\text { (mg/L } \\
\text { as } \mathrm{N} \text { ) }\end{array}$ & $\begin{array}{c}\text { Nitro- } \\
\text { gen, } \\
\text { ammo- } \\
\text { nia } \\
\text { plus } \\
\text { organic, } \\
\text { total } \\
\text { (mg/L } \\
\text { as } \mathrm{N})\end{array}$ & $\begin{array}{c}\text { Nitro- } \\
\text { gen, } \\
\text { ammo- } \\
\text { nia } \\
\text { plus } \\
\text { organic, } \\
\text { dis- } \\
\text { solved } \\
\text { (mg/L } \\
\text { as } \mathrm{N} \text { ) }\end{array}$ & $\begin{array}{c}\text { Phos- } \\
\text { phorus, } \\
\text { total } \\
\text { (mg/L } \\
\text { as P) }\end{array}$ & $\begin{array}{l}\text { Phos- } \\
\text { phorus, } \\
\text { dis- } \\
\text { solved } \\
\text { (mg/L } \\
\text { as P) }\end{array}$ & $\begin{array}{l}\text { Phos- } \\
\text { phorus, } \\
\text { ortho, } \\
\text { dis- } \\
\text { solved } \\
\text { (mg/L } \\
\text { as P) }\end{array}$ \\
\hline \multirow[t]{12}{*}{64} & $07-28-88$ & 1230 & $<0.01$ & $<0.10$ & 0.02 & 0.80 & $<0.20$ & 0.09 & 0.03 & 0.02 \\
\hline & $07-28-88$ & 1235 & $<.01$ & $<.10$ & .02 & 1.0 & .40 & .09 & .03 & .02 \\
\hline & $07-28-88$ & 1830 & $<.01$ & $<.10$ & .03 & .70 & .30 & .10 & .03 & .02 \\
\hline & $07-29-88$ & 0005 & $<.01$ & .10 & .03 & .30 & .50 & .09 & .04 & .02 \\
\hline & $07-29-88$ & 0435 & $<.01$ & .11 & $<.01$ & .70 & .60 & .08 & .04 & .03 \\
\hline & $11-14-88$ & 1530 & $<.01$ & .31 & .03 & .70 & .40 & .07 & .02 & $<.01$ \\
\hline & 03-07-89 & 1230 & $<.01$ & .19 & .02 & 1.6 & .60 & .07 & .02 & .01 \\
\hline & 03-07-89 & 1235 & $<.01$ & .20 & .03 & 1.6 & 1.6 & .06 & .02 & .01 \\
\hline & 03-07-89 & 1330 & $<.01$ & .23 & .03 & 3.7 & 1.7 & .05 & .02 & .02 \\
\hline & $03-07-89$ & 1335 & $<.01$ & .19 & .02 & 1.0 & 1.0 & .05 & .02 & $<.01$ \\
\hline & $05-31-89$ & 1430 & $<.01$ & $<.10$ & .03 & .50 & .40 & .11 & .05 & .04 \\
\hline & $05-31-89$ & 1435 & $<.01$ & $<.10$ & .03 & .30 & .50 & .10 & .06 & .04 \\
\hline \multirow[t]{4}{*}{71} & $07-27-88$ & 0610 & $<.01$ & $<.10$ & .11 & 1.3 & .40 & .21 & .09 & .08 \\
\hline & $11-17-88$ & 1230 & .02 & .44 & .09 & 1.0 & .80 & .54 & .49 & .41 \\
\hline & 03-09-89 & 1100 & .03 & 1.3 & 2.5 & 3.3 & 3.3 & 1.0 & .87 & .81 \\
\hline & 06-01-89 & 1445 & .02 & $<.10$ & .19 & .70 & .90 & .60 & .53 & .49 \\
\hline \multirow[t]{13}{*}{72} & $07-27-88$ & 0745 & $<.01$ & $<.10$ & .03 & .60 & .50 & 1.2 & .04 & .03 \\
\hline & $08-31-88$ & 1025 & -. & $<.01$ & .04 & .80 & -- & .20 & .07 & -. \\
\hline & $10-05-88$ & 1000 & - & $<.01$ & .02 & .40 & -- & .10 & .04 & -- \\
\hline & $11-09-88$ & 1020 & -- & $<.01$ & $<.01$ & -- & -- & .17 & .10 & -- \\
\hline & $11-17-88$ & 1020 & $<.01$ & $<.10$ & .08 & .80 & .70 & .10 & .15 & .13 \\
\hline & $12-21-88$ & 1010 & -- & .21 & .03 & .40 & - & .11 & .10 & -- \\
\hline & $01-25-89$ & 1220 & -- & -- & .08 & - & - & .10 & .06 & -- \\
\hline & $02-15-89$ & 1035 & -- & .29 & $<.01$ & -- & -- & .20 & .02 & -- \\
\hline & 03-09-89 & 1230 & $<.01$ & .12 & .03 & .60 & .60 & .14 & .08 & .07 \\
\hline & 03-15-89 & 1135 & -- & .05 & .05 & -- & -- & .05 & $<.01$ & -- \\
\hline & $04-19-89$ & 0925 & - & .11 & .11 & -- & -- & .13 & $<.01$ & -- \\
\hline & $05-17-89$ & 1320 & -- & $<.01$ & $<.01$ & -- & -- & .23 & .06 & -- \\
\hline & $06-02-89$ & 0945 & $<.01$ & $<.10$ & .03 & 1.6 & .80 & .12 & .08 & .06 \\
\hline
\end{tabular}


Table 8. Results of ancillary chemical analyses of water samples collected July 1988 through June 1989 in the lower Kansas River Basin-Continued

\begin{tabular}{|c|c|c|c|c|c|c|c|c|c|c|}
\hline $\begin{array}{c}\text { Site } \\
\text { map- } \\
\text { index } \\
\text { number } \\
\text { (fig. 10) }\end{array}$ & Date & $\begin{array}{l}\text { Time } \\
\text { (24- } \\
\text { hour) }\end{array}$ & $\begin{array}{c}\text { Nitro- } \\
\text { gen, } \\
\text { nitrite, } \\
\text { dis- } \\
\text { solved } \\
\text { (mg/L } \\
\text { as N) }\end{array}$ & $\begin{array}{c}\text { Nitro- } \\
\text { gen, } \\
\mathrm{NO}_{2+} \\
\mathrm{NO}_{3} \text {, } \\
\text { dis- } \\
\text { solved } \\
\text { (mg/L } \\
\text { as N) }\end{array}$ & $\begin{array}{l}\text { Nitro- } \\
\text { gen, } \\
\text { ammo- } \\
\text { nia, dis- } \\
\text { solved } \\
\text { (mg/L } \\
\text { as } \mathrm{N} \text { ) }\end{array}$ & $\begin{array}{c}\text { Nitro- } \\
\text { gen, } \\
\text { ammo- } \\
\text { nia } \\
\text { plus } \\
\text { organic, } \\
\text { total } \\
\text { (mg/L } \\
\text { as } \mathrm{N} \text { ) }\end{array}$ & $\begin{array}{c}\text { Nitro- } \\
\text { gen, } \\
\text { ammo- } \\
\text { nia } \\
\text { plus } \\
\text { organic, } \\
\text { dis- } \\
\text { solved } \\
\text { (mg/L } \\
\text { as } \mathrm{N} \text { ) }\end{array}$ & $\begin{array}{c}\text { Phos- } \\
\text { phorus, } \\
\text { total } \\
\text { (mg/L } \\
\text { as P) }\end{array}$ & $\begin{array}{c}\text { Phos- } \\
\text { phorus, } \\
\text { dis- } \\
\text { solved } \\
\text { (mg/L } \\
\text { as P) }\end{array}$ & $\begin{array}{c}\text { Phos- } \\
\text { phorus, } \\
\text { ortho, } \\
\text { dis- } \\
\text { solved } \\
\text { (mg/L } \\
\text { as P) }\end{array}$ \\
\hline \multirow[t]{11}{*}{73} & $07-27-88$ & 0810 & 0.01 & 0.18 & 0.17 & 0.80 & 0.50 & 1.8 & 0.12 & 0.10 \\
\hline & $11-17-88$ & 1300 & .05 & .75 & 1.5 & 3.0 & 2.6 & 1.3 & .98 & .95 \\
\hline & $11-17-88$ & 1305 & .05 & .70 & 1.5 & 3.1 & 2.6 & 1.2 & 1.0 & .96 \\
\hline & $11-17-88$ & 1345 & .05 & .69 & 1.4 & 3.0 & 2.5 & 1.2 & 1.0 & .95 \\
\hline & $11-17-88$ & 1350 & .05 & .69 & 1.4 & 3.1 & 2.5 & 1.2 & 1.0 & .94 \\
\hline & 03-09-89 & 1400 & .06 & .87 & 1.1 & 2.0 & 1.5 & .87 & .73 & .66 \\
\hline & 03-09-89 & 1405 & .06 & .86 & .99 & 1.2 & 1.2 & .87 & .75 & .67 \\
\hline & 03-09-89 & 1440 & .06 & .85 & 1.0 & 1.6 & - & .87 & .73 & .67 \\
\hline & 03-09-89 & 1445 & .06 & .85 & .98 & 2.2 & 1.4 & .82 & .73 & .67 \\
\hline & $06-02-89$ & 1100 & .06 & .15 & .15 & 1.0 & .90 & .57 & .58 & .51 \\
\hline & $06-02-89$ & 1105 & .06 & .15 & .15 & .80 & .90 & .61 & .54 & .52 \\
\hline \multirow[t]{14}{*}{74} & $07-29-88$ & 1020 & $<.01$ & $<.10$ & .67 & 1.3 & 1.3 & .32 & .03 & .06 \\
\hline & $08-10-88$ & 1300 & - & $<.01$ & .71 & 1.1 & -- & .79 & .10 & -- \\
\hline & $08-31-88$ & 1320 & -- & $<.01$ & .06 & .70 & -- & .09 & .06 & - \\
\hline & $10-05-88$ & 1300 & - & .14 & $<.01$ & .40 & -- & .05 & $<.01$ & -- \\
\hline & $11-09-88$ & 1310 & - & .17 & .03 & -- & -- & .03 & .03 & -- \\
\hline & $11-15-88$ & 1430 & $<.01$ & .17 & .05 & .60 & .60 & .05 & .02 & .02 \\
\hline & $12-21-88$ & 1315 & -- & .39 & .06 & .50 & -- & .02 & $<.01$ & -- \\
\hline & $01-25-89$ & 0845 & -- & $<.01$ & .12 & -- & -- & .07 & $<.01$ & -- \\
\hline & $02-15-89$ & 1335 & -- & $<.01$ & .22 & -- & -- & .16 & .06 & -- \\
\hline & 03-07-89 & 0800 & $<.01$ & $<.10$ & .08 & .60 & .60 & .03 & .01 & .02 \\
\hline & $03-15-89$ & 0845 & -- & .10 & .09 & -- & -- & $<.01$ & $<.01$ & -- \\
\hline & $04-19-89$ & 1235 & -- & $<.01$ & .04 & -- & -- & $<.01$ & $<.01$ & -- \\
\hline & $05-17-89$ & 1045 & -- & .02 & .13 & -- & -- & .06 & .09 & -- \\
\hline & $05-31-89$ & 1630 & .01 & .25 & .15 & .70 & .80 & .05 & .03 & .01 \\
\hline
\end{tabular}


Table 8. Results of ancillary chemical analyses of water samples collected July 1988 through June 1989 in the lower Kansas River Basin-Continued

\begin{tabular}{|c|c|c|c|c|c|c|c|c|c|c|}
\hline $\begin{array}{c}\text { Site } \\
\text { map- } \\
\text { index } \\
\text { number } \\
\text { (fig. 10) }\end{array}$ & Date & $\begin{array}{l}\text { Time } \\
\text { (24- } \\
\text { hour) }\end{array}$ & $\begin{array}{l}\text { Nitro- } \\
\text { gen, } \\
\text { nitrite, } \\
\text { dis- } \\
\text { solved } \\
\text { (mg/L } \\
\text { as } \mathrm{N} \text { ) }\end{array}$ & $\begin{array}{l}\text { Nitro- } \\
\text { gen, } \\
\mathrm{NO}_{2+} \\
\mathrm{NO}_{3}, \\
\text { dis- } \\
\text { solved } \\
\text { (mg/L } \\
\text { as N) }\end{array}$ & $\begin{array}{l}\text { Nitro- } \\
\text { gen, } \\
\text { ammo- } \\
\text { nia, dis- } \\
\text { solved } \\
\text { (mg/L } \\
\text { as } \mathrm{N} \text { ) }\end{array}$ & $\begin{array}{c}\text { Nitro- } \\
\text { gen, } \\
\text { ammo- } \\
\text { nia } \\
\text { plus } \\
\text { organic, } \\
\text { total } \\
(\mathrm{mg} / \mathrm{L} \\
\text { as } \mathrm{N})\end{array}$ & $\begin{array}{c}\text { Nitro- } \\
\text { gen, } \\
\text { ammo- } \\
\text { nia } \\
\text { plus } \\
\text { organic, } \\
\text { dis- } \\
\text { solved } \\
\text { (mg/L } \\
\text { as } \mathrm{N} \text { ) }\end{array}$ & $\begin{array}{c}\text { Phos- } \\
\text { phorus, } \\
\text { total } \\
\text { (mg/L } \\
\text { as P) }\end{array}$ & $\begin{array}{c}\text { Phos- } \\
\text { phorus, } \\
\text { dis- } \\
\text { solved } \\
\text { (mg/L } \\
\text { as P) }\end{array}$ & $\begin{array}{l}\text { Phos- } \\
\text { phorus, } \\
\text { ortho, } \\
\text { dis- } \\
\text { solved } \\
\text { (mg/L } \\
\text { as P) }\end{array}$ \\
\hline \multirow[t]{10}{*}{76} & $07-27-88$ & 1805 & $<0.01$ & $<.010$ & 0.03 & 1.3 & 0.60 & 0.20 & 0.03 & 0.01 \\
\hline & $07-28-88$ & 0025 & $<.01$ & $<.10$ & .03 & 1.4 & .40 & .10 & .04 & .01 \\
\hline & $07-28-88$ & 0615 & $<.01$ & $<.10$ & .03 & 1.4 & $<.20$ & .26 & .03 & .01 \\
\hline & $07-28-88$ & 1215 & $<.01$ & $<.10$ & .01 & 1.0 & .40 & .20 & .03 & .01 \\
\hline & $07-28-88$ & 1220 & $<.01$ & $<.10$ & .01 & 1.3 & .20 & .24 & .02 & .01 \\
\hline & $11-14-88$ & 0600 & .02 & .57 & .08 & .80 & .60 & .25 & .19 & .16 \\
\hline & $11-14-88$ & 0610 & .02 & .56 & .08 & .80 & .60 & .24 & .19 & .16 \\
\hline & $11-14-88$ & 1000 & .03 & .54 & .10 & .90 & .70 & .28 & .22 & .20 \\
\hline & $11-14-88$ & 1005 & .03 & .54 & .10 & .90 & .70 & .29 & .20 & .19 \\
\hline & $11-14-88$ & 1400 & .03 & .54 & .19 & .80 & .60 & .28 & .21 & .19 \\
\hline & $11-14-88$ & 1410 & .03 & .58 & .20 & 1.0 & .80 & .28 & .20 & .19 \\
\hline & $11-14-88$ & 1800 & .03 & .49 & .14 & 1.0 & .70 & .20 & .19 & .17 \\
\hline & $11-14-88$ & 1805 & .03 & .50 & .14 & .80 & .60 & .26 & .19 & .17 \\
\hline & $11-14-88$ & 2200 & .03 & .47 & .10 & .80 & .70 & .25 & .18 & .16 \\
\hline & $11-14-88$ & 2205 & .03 & .46 & .10 & 1.0 & .60 & .23 & .18 & .16 \\
\hline & $11-15-88$ & 0200 & .02 & .42 & .04 & .70 & .70 & .20 & .14 & .13 \\
\hline & $11-15-88$ & 0205 & .02 & .42 & .05 & .80 & .80 & .19 & .13 & .13 \\
\hline & $11-15-88$ & 0605 & .03 & .50 & .06 & .90 & .60 & .24 & .22 & .19 \\
\hline & 03-07-89 & 0615 & .04 & .61 & .38 & 1.0 & .80 & .35 & .25 & .21 \\
\hline & 03-07-89 & 1010 & .03 & .56 & .46 & 1.1 & 1.0 & .32 & .25 & .22 \\
\hline & $03-07-89$ & 1015 & .03 & .57 & .47 & 1.3 & .90 & .32 & .26 & .21 \\
\hline & $03-07-89$ & 1400 & .03 & .52 & .56 & 1.2 & 1.0 & .31 & .22 & .19 \\
\hline & $03-07-89$ & 1405 & .03 & .52 & .37 & 1.1 & 1.0 & .29 & .22 & .19 \\
\hline & $03-07-89$ & 1410 & .03 & .57 & .57 & 1.3 & .70 & .29 & .22 & .19 \\
\hline & $03-07-89$ & 1800 & .03 & .53 & .59 & 1.1 & 1.1 & .29 & .22 & .19 \\
\hline & 03-07-89 & 1805 & .03 & .53 & .61 & 1.4 & 1.0 & .30 & .23 & .20 \\
\hline & $03-07-89$ & 2200 & .03 & .55 & .57 & 1.5 & 1.0 & .32 & .24 & .20 \\
\hline & 03-07-89 & 2205 & .03 & .56 & .54 & .80 & .80 & .35 & .23 & .21 \\
\hline & 03-08-89 & 0200 & .03 & .54 & .36 & 1.2 & .70 & .27 & .20 & .17 \\
\hline
\end{tabular}


Table 8. Results of ancillary chemical analyses of water samples collected July 1988 through June 1989 in the lower Kansas River Basin-Continued

\begin{tabular}{|c|c|c|c|c|c|c|c|c|c|c|}
\hline $\begin{array}{c}\text { Site } \\
\text { map- } \\
\text { index } \\
\text { number } \\
\text { (fig. 10) }\end{array}$ & Date & $\begin{array}{l}\text { Time } \\
\text { (24- } \\
\text { hour) }\end{array}$ & $\begin{array}{l}\text { Nitro- } \\
\text { gen, } \\
\text { nitrite, } \\
\text { dis- } \\
\text { solved } \\
\text { (mg/L } \\
\text { as } \mathrm{N} \text { ) }\end{array}$ & $\begin{array}{l}\text { Nitro- } \\
\text { gen, } \\
\mathrm{NO}_{2+} \\
\mathrm{NO}_{3} \text {, } \\
\text { dis- } \\
\text { solved } \\
\text { (mg/L } \\
\text { as } \mathrm{N} \text { ) }\end{array}$ & $\begin{array}{l}\text { Nitro- } \\
\text { gen, } \\
\text { ammo- } \\
\text { nia, dis- } \\
\text { solved } \\
\text { (mg/L } \\
\text { as N) }\end{array}$ & $\begin{array}{l}\text { Nitro- } \\
\text { gen, } \\
\text { ammo- } \\
\text { nia } \\
\text { plus } \\
\text { organic, } \\
\text { total } \\
\text { (mg/L } \\
\text { as } \mathrm{N})\end{array}$ & $\begin{array}{l}\text { Nitro- } \\
\text { gen, } \\
\text { ammo- } \\
\text { nia } \\
\text { plus } \\
\text { organic, } \\
\text { dis- } \\
\text { solved } \\
\text { (mg/L } \\
\text { as } \mathrm{N})\end{array}$ & $\begin{array}{c}\text { Phos- } \\
\text { phorus, } \\
\text { total } \\
\text { (mg/L } \\
\text { as P) }\end{array}$ & $\begin{array}{c}\text { Phos- } \\
\text { phorus, } \\
\text { dis- } \\
\text { solved } \\
\text { (mg/L } \\
\text { as P) }\end{array}$ & $\begin{array}{c}\text { Phos- } \\
\text { phorus, } \\
\text { ortho, } \\
\text { dis- } \\
\text { solved } \\
\text { (mg/L } \\
\text { as P) }\end{array}$ \\
\hline \multirow[t]{4}{*}{76} & $03-08-89$ & 0205 & 0.03 & 0.54 & 0.37 & 1.1 & 1.0 & 0.27 & 0.20 & 0.17 \\
\hline & $03-08-89$ & 0600 & .04 & .62 & .38 & 1.8 & .80 & .33 & .22 & .19 \\
\hline & 03-08-89 & 0605 & .04 & .66 & .42 & 1.4 & 1.4 & .33 & .24 & .19 \\
\hline & $05-31-89$ & 1845 & .19 & .97 & .71 & 1.2 & 1.2 & .33 & .28 & .25 \\
\hline \multirow[t]{5}{*}{77} & $07-27-88$ & 1825 & $<.01$ & $<.10$ & .02 & 1.2 & .40 & .15 & $<.01$ & .01 \\
\hline & $07-28-88$ & 0605 & $<.01$ & $<.10$ & .01 & 1.8 & .30 & .14 & .03 & .01 \\
\hline & $11-14-88$ & 1030 & .02 & .58 & .12 & .70 & .70 & .20 & .14 & .13 \\
\hline & $03-07-89$ & 1345 & .03 & .48 & .51 & 1.2 & 1.0 & .27 & .18 & .16 \\
\hline & 06-03-89 & 0935 & .15 & .87 & .13 & .50 & .60 & .26 & .15 & .14 \\
\hline \multirow[t]{4}{*}{78} & $07-28-88$ & 0515 & .01 & $<.10$ & .04 & 1.2 & 1.0 & .26 & .08 & .06 \\
\hline & $11-14-88$ & 1440 & .03 & .84 & .11 & .90 & .20 & .31 & .30 & .31 \\
\hline & $03-07-89$ & 1030 & .07 & 1.1 & .47 & 1.3 & 1.1 & .41 & .30 & .26 \\
\hline & $06-01-89$ & 1800 & .20 & .98 & .41 & 1.1 & 1.0 & .70 & .61 & .43 \\
\hline \multirow[t]{3}{*}{79} & $07-29-88$ & 0645 & $<.01$ & .21 & .04 & .50 & .40 & .09 & .03 & .01 \\
\hline & $03-07-89$ & 1600 & $<.01$ & $<.10$ & .04 & .60 & .50 & .05 & .01 & $<.01$ \\
\hline & $05-31-89$ & 0930 & $<.01$ & $<.10$ & .05 & .80 & .80 & .10 & .05 & .02 \\
\hline \multirow[t]{10}{*}{83} & $07-29-88$ & 0530 & $<.01$ & .11 & .03 & 1.0 & .80 & .04 & $<.01$ & .02 \\
\hline & $08-10-88$ & 1500 & -- & $<.01$ & .14 & .60 & -- & .44 & $<.01$ & -- \\
\hline & $08-31-88$ & 1500 & -- & $<.01$ & .04 & 1.1 & -- & .16 & .05 & -- \\
\hline & $10-05-88$ & 1500 & - & .18 & .04 & .60 & -- & .04 & $<.01$ & -- \\
\hline & $11-09-88$ & 1555 & -- & .04 & $<.01$ & -- & -- & .07 & .03 & -- \\
\hline & $11-16-88$ & 1520 & -- & -- & -- & -- & -- & -- & -- & -- \\
\hline & $12-21-88$ & 1515 & -- & .04 & .03 & .50 & -- & .03 & $<.01$ & -- \\
\hline & $01-25-89$ & 1605 & -- & .64 & .25 & -- & -- & -- & .02 & -- \\
\hline & $02-15-89$ & 1535 & -- & .09 & .04 & -- & -- & .17 & .20 & -- \\
\hline & $03-07-89$ & 1300 & $<.01$ & .15 & .04 & .60 & .50 & .04 & .01 & .01 \\
\hline
\end{tabular}


Table 8. Results of ancillary chemical analyses of water samples collected July 1988 through June 1989 in the lower Kansas River Basin-Continued

\begin{tabular}{|c|c|c|c|c|c|c|c|c|c|c|}
\hline $\begin{array}{c}\text { Site } \\
\text { map- } \\
\text { index } \\
\text { number } \\
\text { (fig. 10) }\end{array}$ & Date & $\begin{array}{l}\text { Time } \\
\text { (24- } \\
\text { hour) }\end{array}$ & $\begin{array}{c}\text { Nitro- } \\
\text { gen, } \\
\text { nitrite, } \\
\text { dis- } \\
\text { solved } \\
\text { (mg/L } \\
\text { as N) }\end{array}$ & $\begin{array}{c}\text { Nitro- } \\
\text { gen, } \\
\mathrm{NO}_{2+} \\
\mathrm{NO}_{3} \text {, } \\
\text { dis- } \\
\text { solved } \\
\text { (mg/L } \\
\text { as } \mathrm{N} \text { ) }\end{array}$ & $\begin{array}{l}\text { Nitro- } \\
\text { gen, } \\
\text { ammo- } \\
\text { nia, dis- } \\
\text { solved } \\
\text { (mg/L } \\
\text { as } \mathrm{N} \text { ) }\end{array}$ & $\begin{array}{c}\text { Nitro- } \\
\text { gen, } \\
\text { ammo- } \\
\text { nia } \\
\text { plus } \\
\text { organic, } \\
\text { total } \\
\text { (mg/L } \\
\text { as } \mathrm{N})\end{array}$ & $\begin{array}{c}\text { Nitro- } \\
\text { gen, } \\
\text { ammo- } \\
\text { nia } \\
\text { plus } \\
\text { organic, } \\
\text { dis- } \\
\text { solved } \\
\text { (mg/L } \\
\text { as } \mathrm{N} \text { ) }\end{array}$ & $\begin{array}{c}\text { Phos- } \\
\text { phorus, } \\
\text { total } \\
\text { (mg/L } \\
\text { as P) }\end{array}$ & $\begin{array}{c}\text { Phos- } \\
\text { phorus, } \\
\text { dis- } \\
\text { solved } \\
\text { (mg/L } \\
\text { as P) }\end{array}$ & $\begin{array}{c}\text { Phos- } \\
\text { phorus, } \\
\text { ortho, } \\
\text { dis- } \\
\text { solved } \\
\text { (mg/L } \\
\text { as P) }\end{array}$ \\
\hline \multirow[t]{4}{*}{83} & $03-15-89$ & 1435 & -- & 0.11 & 0.05 & - & - & 0.12 & $<0.01$ & -- \\
\hline & $04-19-89$ & 1450 & -- & $<.01$ & .07 & -- & -- & $<.01$ & $<.01$ & - \\
\hline & $05-17-89$ & 0755 & -- & $<.01$ & $<.01$ & -- & -- & .22 & .02 & -- \\
\hline & $05-31-89$ & 1045 & $<0.01$ & $<.10$ & .03 & 0.40 & 0.60 & .04 & .02 & 0.01 \\
\hline \multirow[t]{5}{*}{86} & $07-28-88$ & 0615 & $<.01$ & $<.10$ & .16 & 1.2 & .90 & .13 & .06 & .03 \\
\hline & $07-28-88$ & 0620 & $<.01$ & $<.10$ & .16 & 1.3 & .80 & .13 & .05 & .02 \\
\hline & $07-28-88$ & 1200 & $<.01$ & $<.10$ & .03 & 1.8 & .80 & .25 & .04 & .01 \\
\hline & 03-08-89 & 1200 & $<.01$ & $<.10$ & .01 & .80 & .30 & .07 & .03 & .03 \\
\hline & $06-02-89$ & 1250 & .02 & $<.10$ & .08 & .70 & .90 & .06 & .03 & .02 \\
\hline \multirow[t]{6}{*}{87} & $07-28-88$ & 0445 & $<.01$ & $<.10$ & .03 & 1.0 & 1.0 & .13 & .01 & .03 \\
\hline & $07-28-88$ & 1500 & $<.01$ & $<.10$ & .04 & 1.0 & .80 & .16 & .05 & .04 \\
\hline & $11-14-88$ & 1345 & $<.01$ & $<.10$ & .03 & .90 & .60 & .14 & .05 & .04 \\
\hline & $11-14-88$ & 1350 & $<.01$ & $<.10$ & .03 & 1.3 & .60 & .15 & .04 & .03 \\
\hline & 03-08-89 & 1415 & $<.01$ & $<.10$ & .01 & .60 & .50 & .09 & .03 & .03 \\
\hline & $06-02-89$ & 1530 & .12 & 1.5 & .35 & 1.7 & 1.5 & .19 & .08 & .08 \\
\hline \multirow[t]{13}{*}{88} & $07-27-88$ & 1815 & $<.01$ & $<.10$ & .03 & .90 & .50 & .16 & .04 & .03 \\
\hline & $07-27-88$ & 2400 & $<.01$ & $<.10$ & .01 & 1.4 & .70 & .20 & .02 & .03 \\
\hline & $07-28-88$ & 0610 & $<.01$ & $<.10$ & .02 & .50 & .40 & .18 & .06 & .04 \\
\hline & $07-28-88$ & 1220 & .01 & $<.10$ & .04 & 1.4 & $<.20$ & .20 & .06 & .04 \\
\hline & $07-28-88$ & 1225 & .01 & $<.10$ & .03 & 1.1 & .70 & .15 & .06 & .04 \\
\hline & $11-16-88$ & 1510 & .03 & .71 & .05 & .90 & .60 & .35 & .30 & .26 \\
\hline & $11-16-88$ & 1515 & .03 & .71 & .13 & .90 & .50 & .35 & .30 & .27 \\
\hline & 03-07-89 & 1550 & .04 & 1.1 & .36 & 1.2 & 1.2 & .35 & .23 & .20 \\
\hline & 03-07-89 & 1555 & .04 & 1.0 & .34 & 1.4 & 1.2 & .38 & .25 & .20 \\
\hline & 03-07-89 & 1630 & .04 & -- & .36 & 1.1 & 1.3 & .36 & .27 & .22 \\
\hline & 03-07-89 & 1635 & .04 & 1.1 & .36 & 1.3 & 1.0 & .39 & .28 & .22 \\
\hline & $05-30-89$ & 1905 & .12 & .74 & .03 & .60 & .30 & .53 & .23 & .18 \\
\hline & $05-30-89$ & 1910 & .12 & .73 & .03 & .70 & .60 & .29 & .22 & .18 \\
\hline
\end{tabular}


Table 8. Results of ancillary chemical analyses of water samples collected July 1988 through June 1989 in the lower Kansas River Basin-Continued

\begin{tabular}{|c|c|c|c|c|c|c|c|c|c|c|}
\hline $\begin{array}{c}\text { Site } \\
\text { map- } \\
\text { index } \\
\text { number } \\
\text { (fig. 10) }\end{array}$ & Date & $\begin{array}{l}\text { Time } \\
\text { (24- } \\
\text { hour) }\end{array}$ & $\begin{array}{l}\text { Nitro- } \\
\text { gen, } \\
\text { nitrite, } \\
\text { dis- } \\
\text { solved } \\
\text { (mgh } \\
\text { as } \mathrm{N} \text { ) }\end{array}$ & $\begin{array}{c}\text { Nitro- } \\
\text { gen, } \\
\mathrm{NO}_{2+} \\
\mathrm{NO}_{3}, \\
\text { dis- } \\
\text { solved } \\
\text { (mg/L } \\
\text { as } \mathrm{N} \text { ) }\end{array}$ & $\begin{array}{c}\text { Nitro- } \\
\text { gen, } \\
\text { ammo- } \\
\text { nia, dis- } \\
\text { solved } \\
\text { (mg/l } \\
\text { as } \mathrm{N} \text { ) }\end{array}$ & $\begin{array}{c}\text { Nitro- } \\
\text { gen, } \\
\text { ammo- } \\
\text { nia } \\
\text { plus } \\
\text { organic, } \\
\text { total } \\
\text { (mg/l } \\
\text { as } \mathrm{N} \text { ) }\end{array}$ & $\begin{array}{c}\text { Nitro- } \\
\text { gen, } \\
\text { ammo- } \\
\text { nia } \\
\text { plus } \\
\text { organic, } \\
\text { dis- } \\
\text { solved } \\
\text { (mg/L } \\
\text { as } \mathrm{N} \text { ) }\end{array}$ & $\begin{array}{c}\text { Phos- } \\
\text { phorus, } \\
\text { total } \\
\text { (mg/L } \\
\text { as P) }\end{array}$ & $\begin{array}{c}\text { Phos- } \\
\text { phorus, } \\
\text { dis- } \\
\text { solved } \\
\text { (mg/l } \\
\text { as P) }\end{array}$ & $\begin{array}{l}\text { Phos- } \\
\text { phorus, } \\
\text { ortho, } \\
\text { dis- } \\
\text { solved } \\
\text { (mg/L } \\
\text { as P) }\end{array}$ \\
\hline \multirow[t]{8}{*}{90} & $07-29-88$ & 0510 & 0.72 & 8.8 & -- & 14 & -- & 8.7 & 8.2 & 6.7 \\
\hline & $07-29-88$ & 0515 & .67 & 8.2 & 5.1 & 14 & 7.7 & 8.5 & 7.7 & 6.3 \\
\hline & $11-14-88$ & 1210 & .68 & -- & -- & 7.5 & 6.3 & 6.9 & 6.3 & 5.1 \\
\hline & $11-14-88$ & 1215 & .69 & 5.3 & 4.6 & 7.2 & 7.0 & 6.8 & 6.2 & 4.9 \\
\hline & $11-14-88$ & 1315 & .67 & 6.8 & 4.4 & 6.9 & 6.4 & 7.2 & 6.8 & 5.3 \\
\hline & $11-14-88$ & 1320 & .66 & 6.5 & 4.2 & 7.5 & 6.8 & 7.5 & 6.7 & 5.4 \\
\hline & 03-07-89 & 0925 & .45 & 4.5 & 11 & 3.6 & 13 & 7.3 & 6.7 & 5.4 \\
\hline & $06-02-89$ & 1440 & .62 & 5.3 & 2.0 & 5.9 & 5.0 & 3.4 & 3.7 & 3.2 \\
\hline \multirow[t]{4}{*}{91} & $07-29-88$ & 0615 & .06 & .59 & .77 & .60 & 1.0 & .55 & .34 & .50 \\
\hline & $11-14-88$ & 1000 & .04 & 1.4 & .48 & .80 & .80 & .41 & .30 & .27 \\
\hline & 03-07-89 & 1320 & .04 & .98 & .33 & 1.4 & 1.2 & .43 & .22 & .20 \\
\hline & $06-02-89$ & 1145 & .05 & .25 & .71 & 1.7 & 1.6 & .53 & .36 & .33 \\
\hline
\end{tabular}


Table 8. Results of ancillary chemical analyses of water samples collected July 1988 through June 1989 in the lower Kansas River Basin-Continued

\begin{tabular}{|c|c|c|c|c|c|c|}
\hline $\begin{array}{c}\text { Site } \\
\text { map-index } \\
\text { number } \\
\text { (fig. 10) }\end{array}$ & Date & Time (24-hour) & $\begin{array}{l}\text { Carbon, } \\
\text { organic, total } \\
\text { ( } \mathrm{mg} / \mathrm{L} \text { as C) }\end{array}$ & $\begin{array}{l}\text { Chlorophyll-a } \\
\text { phyto- } \\
\text { plankton, } \\
\text { chromo- } \\
\text { fluorometer } \\
(\mu \mathrm{g} / \mathrm{L})\end{array}$ & $\begin{array}{l}\text { Chlorophyll-b } \\
\text { phyto- } \\
\text { plankton, } \\
\text { chromo- } \\
\text { fluorometer } \\
(\mu \mathrm{g} / \mathrm{L})\end{array}$ & $\begin{array}{c}\text { Sediment, } \\
\text { suspended } \\
\text { (mg/L) }\end{array}$ \\
\hline \multirow[t]{4}{*}{1} & $07-27-88$ & 1300 & 15 & 56 & 9.6 & 121 \\
\hline & $11-15-88$ & 1205 & 10 & 44 & 1.0 & 119 \\
\hline & 03-08-89 & 1000 & 5.2 & 33 & 12 & 18 \\
\hline & $06-01-89$ & 1010 & 9.9 & 110 & 3.8 & 213 \\
\hline \multirow[t]{4}{*}{2} & $07-27-88$ & 1150 & 5.9 & 12 & 3.4 & 137 \\
\hline & $11-15-88$ & 1050 & 5.5 & 4.9 & .1 & 9 \\
\hline & $03-08-89$ & 1140 & 2.8 & 2.7 & .6 & 21 \\
\hline & $06-01-89$ & 0830 & 5.6 & 15 & 1.8 & 34 \\
\hline \multirow[t]{4}{*}{5} & $07-24-88$ & 1020 & 8.6 & 27 & 2.8 & 210 \\
\hline & $11-16-88$ & 0850 & 7.7 & 30 & .6 & 45 \\
\hline & $03-09-89$ & 0930 & 8.2 & 51 & 23 & 20 \\
\hline & $06-02-89$ & 0820 & 7.6 & 78 & 3.5 & 187 \\
\hline \multirow[t]{4}{*}{6} & $07-26-88$ & 0730 & 16 & 45 & 12 & 68 \\
\hline & $11-14-88$ & 1200 & 14 & 16 & 1.0 & 62 \\
\hline & 03-08-89 & 1015 & 17 & 1.1 & .6 & 86 \\
\hline & $05-31-89$ & 1445 & 15 & 63 & 21 & 53 \\
\hline \multirow[t]{6}{*}{9} & $07-26-88$ & 0945 & 9.0 & 12 & 5.0 & 34 \\
\hline & $07-26-88$ & 2150 & -- & -- & -- & -- \\
\hline & $07-27-88$ & 0550 & -- & -- & -- & -- \\
\hline & $11-15-88$ & 0815 & 25 & 11 & .9 & 33 \\
\hline & 03-07-89 & 1015 & 54 & 9.9 & 7.6 & 107 \\
\hline & $05-31-89$ & 0930 & 26 & 71 & 12 & 39 \\
\hline \multirow[t]{5}{*}{10} & $07-26-88$ & 0900 & 11 & 24 & 5.6 & 163 \\
\hline & $07-26-88$ & 1300 & -- & -- & -- & -- \\
\hline & $07-26-88$ & 1700 & -- & -- & -- & -- \\
\hline & $07-26-88$ & 2100 & -- & -- & -- & -- \\
\hline & $07-27-88$ & 0100 & -- & -- & -- & -- \\
\hline
\end{tabular}


Table 8. Results of ancillary chemical analyses of water samples collected July 1988 through June 1989 in the lower Kansas River Basin-Continued

\begin{tabular}{|c|c|c|c|c|c|c|}
\hline $\begin{array}{c}\text { Site } \\
\text { map-index } \\
\text { number } \\
\text { (flg. 10) }\end{array}$ & Date & Time (24-hour) & $\begin{array}{l}\text { Carbon, } \\
\text { organic, total } \\
\text { (mg/L as C) }\end{array}$ & $\begin{array}{l}\text { Chlorophyll-a } \\
\text { phyto- } \\
\text { plankton, } \\
\text { chromo- } \\
\text { fluorometer } \\
(\mu \mathrm{g} / \mathrm{L})\end{array}$ & $\begin{array}{l}\text { Chlorophyll-b } \\
\text { phyto- } \\
\text { plankton, } \\
\text { chromo- } \\
\text { fluorometer } \\
(\mu \mathrm{g} / \mathrm{L})\end{array}$ & $\begin{array}{l}\text { Sediment, } \\
\text { suspended } \\
\text { (mg/L) }\end{array}$ \\
\hline \multirow[t]{5}{*}{10} & $07-27-88$ & 0500 & -- & -- & -- & -- \\
\hline & $07-27-88$ & 0900 & -- & -- & -- & -- \\
\hline & $11-14-88$ & 1345 & 20 & 12 & 1.0 & 52 \\
\hline & 03-07-89 & 1000 & 24 & 1.7 & .8 & 44 \\
\hline & $05-31-89$ & 1330 & 11 & 1.5 & .2 & 10 \\
\hline \multirow[t]{4}{*}{11} & $07-26-88$ & 0845 & 22 & 39 & 9.7 & 251 \\
\hline & $11-14-88$ & 1050 & 3.5 & 4.9 & .3 & 70 \\
\hline & 03-08-89 & 1215 & 14 & 2.2 & .3 & 98 \\
\hline & $05-31-89$ & 1600 & 7.1 & 45 & 4.4 & 145 \\
\hline \multirow[t]{9}{*}{12} & $07-26-88$ & 1000 & 29 & 68 & 21 & 222 \\
\hline & $07-26-88$ & 1005 & -- & -- & -- & -- \\
\hline & $07-26-88$ & 1400 & -- & -- & -- & -- \\
\hline & $07-26-88$ & 1800 & -- & -- & -- & -- \\
\hline & $07-26-88$ & 2200 & - & -- & -- & -- \\
\hline & $07-27-88$ & 0200 & -- & -- & -- & -- \\
\hline & $07-27-88$ & 0600 & -- & -- & -- & -- \\
\hline & $11-14-88$ & 0845 & 6.6 & 100 & 3.0 & 56 \\
\hline & 03-08-89 & 1130 & 9.9 & 1.6 & .3 & 73 \\
\hline \multirow[t]{7}{*}{16} & $07-26-88$ & 0630 & 9.2 & 1.6 & $<.3$ & 18 \\
\hline & $07-26-88$ & 1800 & -- & -- & -- & -- \\
\hline & $07-27-88$ & 1215 & -- & -- & -- & -- \\
\hline & $09-06-88$ & 1145 & -- & -- & -- & -- \\
\hline & $11-15-88$ & 1015 & 18 & 4.6 & .7 & 2 \\
\hline & $03-07-89$ & 1600 & 18 & 1.3 & 1.8 & 21 \\
\hline & $05-30-89$ & 1145 & 15 & 17 & 4.5 & 3 \\
\hline \multirow[t]{5}{*}{17} & $07-25-88$ & 1230 & 9.3 & 50 & 14 & 194 \\
\hline & $07-26-88$ & 1300 & -- & -- & -- & -- \\
\hline & $07-26-88$ & 1700 & -- & -- & -- & -- \\
\hline & $07-26-88$ & 2100 & -- & -- & -- & -- \\
\hline & $07-27-88$ & 0100 & -- & -- & -- & -- \\
\hline
\end{tabular}


Table 8. Results of ancillary chemical analyses of water samples collected July 1988 through June 1989 in the lower Kansas River Basin-Continued

\begin{tabular}{|c|c|c|c|c|c|c|}
\hline $\begin{array}{c}\text { Site } \\
\text { map-index } \\
\text { number } \\
\text { (fig. 10) }\end{array}$ & Date & Time (24-hour) & $\begin{array}{l}\text { Carbon, } \\
\text { organic, total } \\
\text { (mg/L as C) }\end{array}$ & $\begin{array}{l}\text { Chlorophyll-a } \\
\text { phyto- } \\
\text { plankton, } \\
\text { chromo- } \\
\text { fluorometer } \\
(\mu g / L)\end{array}$ & $\begin{array}{l}\text { Chlorophyll-b } \\
\text { phyto- } \\
\text { plankton, } \\
\text { chromo- } \\
\text { fluorometer } \\
(\mu \mathrm{g} / \mathrm{L})\end{array}$ & $\begin{array}{l}\text { Sediment, } \\
\text { suspended } \\
\text { (mg/L) }\end{array}$ \\
\hline \multirow[t]{5}{*}{17} & $07-27-88$ & 0500 & - & -- & -- & -- \\
\hline & $07-27-88$ & 0900 & - & - & -- & - \\
\hline & $07-27-88$ & 1300 & -- & - & -- & -- \\
\hline & $11-15-88$ & 1130 & 13 & 2.5 & 0.2 & 65 \\
\hline & 03-07-89 & 1300 & 12 & 1.4 & .7 & 48 \\
\hline \multirow[t]{4}{*}{18} & $07-25-88$ & 1130 & 13 & 7.4 & 1.6 & 175 \\
\hline & $11-15-88$ & 1230 & 9.2 & 5.4 & .3 & 42 \\
\hline & 03-07-89 & 1445 & 9.5 & .8 & $<.5$ & 183 \\
\hline & $05-30-89$ & 1445 & 13 & 57 & 17 & 87 \\
\hline \multirow[t]{4}{*}{19} & $07-25-88$ & 1200 & 14 & 43 & 12 & 308 \\
\hline & $11-15-88$ & 1300 & 5.3 & 8.9 & .3 & 29 \\
\hline & $03-07-89$ & 1245 & 7.5 & .7 & .4 & 24 \\
\hline & $05-31-89$ & 1100 & 25 & 29 & 3.1 & 525 \\
\hline \multirow[t]{5}{*}{21} & $07-26-88$ & 1010 & 8.3 & 14 & 3.1 & 66 \\
\hline & $09-06-88$ & 1440 & -- & - & - & -- \\
\hline & $11-14-88$ & 1425 & 9.5 & 5.5 & $<.1$ & 21 \\
\hline & $03-07-89$ & 1145 & 10 & 7.4 & 1.1 & 76 \\
\hline & $05-31-89$ & 1245 & 12 & 10 & 1.0 & 60 \\
\hline \multirow[t]{10}{*}{23} & $07-25-88$ & 1245 & 18 & 31 & 7.0 & 211 \\
\hline & $08-08-88$ & 1115 & -- & -- & -- & 179 \\
\hline & $08-31-88$ & 1400 & -- & -- & -- & 74 \\
\hline & $10-12-88$ & 1230 & - & - & - & 106 \\
\hline & $11-07-88$ & 1200 & -- & -- & -- & 14 \\
\hline & $11-15-88$ & 1420 & 4.0 & 5.3 & .1 & - \\
\hline & $12-06-88$ & 1000 & -- & -- & -- & 9 \\
\hline & 01-03-89 & 1130 & - & - & -- & 12 \\
\hline & $02-07-89$ & 1000 & -- & -- & -- & 12 \\
\hline & 03-07-89 & 1400 & -- & -- & - & 41 \\
\hline
\end{tabular}


Table 8. Results of ancillary chemical analyses of water samples collected July 1988 through June 1989 in the lower Kansas River Basin-Continued

\begin{tabular}{|c|c|c|c|c|c|c|}
\hline $\begin{array}{c}\text { Site } \\
\text { map-index } \\
\text { number } \\
\text { (fig. 10) }\end{array}$ & Date & Time (24-hour) & $\begin{array}{c}\text { Carbon, } \\
\text { organic, total } \\
\text { (mg/L as C) }\end{array}$ & $\begin{array}{l}\text { Chlorophyll-a } \\
\text { phyto- } \\
\text { plankton, } \\
\text { chromo- } \\
\text { fluorometer } \\
(\mu g / L)\end{array}$ & $\begin{array}{c}\text { Chlorophyll-b } \\
\text { phyto- } \\
\text { plankton, } \\
\text { chromo- } \\
\text { fluorometer } \\
(\mu \mathrm{g} / \mathrm{L})\end{array}$ & $\begin{array}{l}\text { Sediment, } \\
\text { suspended } \\
\text { (mg/L) }\end{array}$ \\
\hline \multirow[t]{4}{*}{23} & $03-08-89$ & 1345 & 8.4 & 2.1 & 0.8 & 84 \\
\hline & 04-04-89 & 0955 & -- & -- & -- & 120 \\
\hline & $05-02-89$ & 0830 & -- & -- & -- & 43 \\
\hline & $05-31-89$ & 1430 & 11 & 29 & 4.3 & 258 \\
\hline \multirow[t]{5}{*}{26} & $07-25-88$ & 1030 & 13 & 110 & 9.4 & 205 \\
\hline & 09-07-88 & 0840 & -- & -- & -- & -- \\
\hline & $11-14-88$ & 1530 & 4.8 & 20 & .4 & 21 \\
\hline & 03-08-89 & 1500 & 11 & .5 & .4 & 72 \\
\hline & $05-31-89$ & 1300 & 13 & 43 & 3.7 & 413 \\
\hline \multirow[t]{4}{*}{27} & $07-25-88$ & 1325 & 17 & 19 & 5.4 & 201 \\
\hline & $11-15-88$ & 1110 & 11 & 29 & 3.5 & 75 \\
\hline & 03-07-89 & 1345 & 22 & 3.1 & .6 & 53 \\
\hline & $05-30-89$ & 1445 & 12 & 50 & 11 & 128 \\
\hline \multirow[t]{4}{*}{28} & $07-25-88$ & 0600 & 18 & 12 & 2.5 & 353 \\
\hline & $11-14-88$ & 1445 & 7.3 & .9 & $<.1$ & 30 \\
\hline & 03-07-89 & 0855 & 10 & .9 & $<.2$ & 32 \\
\hline & $05-31-89$ & 1215 & 9.9 & 71 & 6.2 & 155 \\
\hline \multirow[t]{4}{*}{29} & $07-25-88$ & 0630 & 9.9 & 52 & 7.0 & 196 \\
\hline & $11-14-88$ & 1200 & 3.6 & 1.4 & $<.1$ & 52 \\
\hline & 03-07-89 & 1240 & 7.5 & .6 & $<.2$ & 29 \\
\hline & $05-31-89$ & 1100 & 8.0 & 34 & 3.2 & 122 \\
\hline \multirow[t]{10}{*}{31} & $07-25-88$ & 1330 & -- & -- & -- & -- \\
\hline & $07-25-88$ & 1335 & -- & -- & -- & -- \\
\hline & $07-25-88$ & 1730 & -- & -- & -- & -- \\
\hline & $07-25-88$ & 2100 & -- & -- & -- & -- \\
\hline & $07-26-88$ & 0200 & -- & -- & -- & -- \\
\hline & $07-26-88$ & 0510 & 12 & 14 & 3.6 & 58 \\
\hline & $07-26-88$ & 0915 & -- & -- & -- & -- \\
\hline & $11-14-88$ & 1045 & 8.9 & .7 & $<.1$ & 2 \\
\hline & 03-08-89 & 0900 & 17 & 1.7 & $<.2$ & 10 \\
\hline & $05-30-89$ & 1630 & 8.3 & 14 & 6.5 & 6 \\
\hline
\end{tabular}


Table 8. Results of ancillary chemical analyses of water samples collected July 1988 through June 1989 in the lower Kansas River Basin-Continued

\begin{tabular}{|c|c|c|c|c|c|c|}
\hline $\begin{array}{c}\text { Site } \\
\text { map-index } \\
\text { number } \\
\text { (fig. 10) }\end{array}$ & Date & Time (24-hour) & $\begin{array}{l}\text { Carbon, } \\
\text { organic, total } \\
\text { (mg/L as C) }\end{array}$ & $\begin{array}{l}\text { Chlorophyll-a } \\
\text { phyto- } \\
\text { plankton, } \\
\text { chromo- } \\
\text { fluorometer } \\
(\mu \mathrm{g} / \mathrm{L})\end{array}$ & $\begin{array}{c}\text { Chlorophyll-b } \\
\text { phyto- } \\
\text { plankton, } \\
\text { chromo- } \\
\text { fluorometer } \\
(\mu \mathrm{g} / \mathrm{L})\end{array}$ & $\begin{array}{l}\text { Sediment, } \\
\text { suspended } \\
\text { (mg/L) }\end{array}$ \\
\hline \multirow[t]{29}{*}{32} & $07-25-88$ & 1330 & 12 & 47 & 9.2 & 262 \\
\hline & $07-25-88$ & 1700 & 12 & 39 & 8.8 & 242 \\
\hline & $07-25-88$ & 2100 & 12 & 36 & 8.0 & 215 \\
\hline & $07-26-88$ & 0115 & 13 & -- & -- & 210 \\
\hline & $07-26-88$ & 0950 & 12 & -- & -- & 253 \\
\hline & $07-27-88$ & 0510 & 9.4 & 80 & 13 & 158 \\
\hline & $11-14-88$ & 0900 & 5.7 & 25 & 1.0 & 29 \\
\hline & $11-14-88$ & 0905 & -- & -- & -- & -- \\
\hline & $11-14-88$ & 1300 & -- & -- & -- & -- \\
\hline & $11-14-88$ & 1305 & -- & -- & -- & -- \\
\hline & $11-14-88$ & 1700 & -- & -- & -- & -- \\
\hline & $11-14-88$ & 1705 & -- & -- & -- & -- \\
\hline & $11-14-88$ & 2100 & -- & -- & -- & -- \\
\hline & $11-14-88$ & 2105 & -- & -- & -- & -- \\
\hline & $11-15-88$ & 0100 & -- & -- & -- & -- \\
\hline & $11-15-88$ & 0105 & -- & -- & -- & -- \\
\hline & $11-15-88$ & 0500 & -- & -- & -- & -- \\
\hline & $11-15-88$ & 0505 & -- & -- & -- & -- \\
\hline & $11-15-88$ & 0900 & -- & -- & -- & -- \\
\hline & $11-15-88$ & 0905 & -- & -- & -- & -- \\
\hline & 03-07-89 & 1000 & 11 & 1.0 & .5 & 59 \\
\hline & 03-07-89 & 1005 & -- & -- & -- & -- \\
\hline & 03-07-89 & 1400 & -. & -- & -- & -- \\
\hline & 03-07-89 & 1405 & -- & -- & -- & -- \\
\hline & 03-07-89 & 1800 & -- & -- & -- & -- \\
\hline & 03-07-89 & 1805 & -- & -- & -- & -- \\
\hline & $03-07-89$ & 2200 & -- & -- & -- & -- \\
\hline & $03-07-89$ & 2205 & -- & -- & -- & -- \\
\hline & $03-08-89$ & 0200 & -- & -- & -- & -- \\
\hline
\end{tabular}


Table 8. Results of ancillary chemical analyses of water samples collected July 1988 through June 1989 in the lower Kansas River Basin-Continued

\begin{tabular}{|c|c|c|c|c|c|c|}
\hline $\begin{array}{c}\text { Site } \\
\text { map-index } \\
\text { number } \\
\text { (fig. 10) }\end{array}$ & Date & Time (24-hour) & $\begin{array}{c}\text { Carbon, } \\
\text { organic, total } \\
\text { (mg/L as C) }\end{array}$ & $\begin{array}{l}\text { Chlorophyll-a } \\
\text { phyto- } \\
\text { plankton, } \\
\text { chromo- } \\
\text { fluorometer } \\
(\mu \mathrm{g} / \mathrm{L})\end{array}$ & $\begin{array}{c}\text { Chlorophyll-b } \\
\text { phyto- } \\
\text { plankton, } \\
\text { chromo- } \\
\text { fluorometer } \\
(\mu \mathrm{g} / \mathrm{L})\end{array}$ & $\begin{array}{l}\text { Sediment, } \\
\text { suspended } \\
\text { (mg/L) }\end{array}$ \\
\hline \multirow[t]{19}{*}{32} & 03-08-89 & 0205 & -- & -- & -- & -- \\
\hline & 03-08-89 & 0600 & -- & -- & -- & -- \\
\hline & $03-08-89$ & 0605 & -- & -- & -- & -- \\
\hline & 03-08-89 & 1000 & -- & -- & -- & -- \\
\hline & 03-08-89 & 1005 & -- & -- & -- & -- \\
\hline & $05-30-89$ & 1000 & 13 & 77 & 9.1 & 101 \\
\hline & $05-30-89$ & 1005 & -- & -- & -- & -- \\
\hline & $05-30-89$ & 1500 & -- & -- & -- & -- \\
\hline & $05-30-89$ & 1505 & -- & -- & -- & -- \\
\hline & $05-30-89$ & 1800 & -- & -- & - & -- \\
\hline & $05-30-89$ & 1810 & -- & -- & -- & -- \\
\hline & $05-30-89$ & 2200 & -- & -- & -- & -- \\
\hline & $05-30-89$ & 2205 & -- & -- & -- & -- \\
\hline & $05-31-89$ & 0200 & -- & -- & -- & - \\
\hline & $05-31-89$ & 0210 & -- & -- & -- & -- \\
\hline & $05-31-89$ & 0600 & -- & -- & -- & -- \\
\hline & $05-31-89$ & 0610 & -- & -- & -- & -- \\
\hline & $05-31-89$ & 1000 & - & -- & -- & -- \\
\hline & $05-31-89$ & 1005 & -- & -- & -- & -- \\
\hline \multirow[t]{12}{*}{34} & $07-25-88$ & 0650 & 10 & 7.1 & .6 & 123 \\
\hline & $07-26-88$ & 0915 & -- & -- & -- & -- \\
\hline & $07-26-88$ & 1045 & -- & -- & -- & -- \\
\hline & $07-26-88$ & 1445 & - & -- & - & - \\
\hline & $07-26-88$ & 1450 & -- & -- & -- & -- \\
\hline & $07-26-88$ & 1845 & -- & -- & -- & -- \\
\hline & $07-26-88$ & 2230 & -- & -- & -- & -- \\
\hline & $07-27-88$ & 0230 & - & -- & - & -- \\
\hline & $07-27-88$ & 0635 & -- & -- & -- & - \\
\hline & $11-14-88$ & 1020 & 6.0 & 5.3 & .3 & 111 \\
\hline & 03-07-89 & 1520 & 5.5 & 2.9 & .6 & 54 \\
\hline & $05-30-89$ & 1345 & 6.7 & 16 & 2.1 & 145 \\
\hline
\end{tabular}


Table 8. Results of ancillary chemical analyses of water samples collected July 1988 through June 1989 in the lower Kansas River Basin-Continued

\begin{tabular}{|c|c|c|c|c|c|c|}
\hline $\begin{array}{c}\text { Site } \\
\text { map-index } \\
\text { number } \\
\text { (fig. 10) }\end{array}$ & Date & Time (24-hour) & $\begin{array}{c}\text { Carbon, } \\
\text { organic, total } \\
\text { (mg/L as C) }\end{array}$ & $\begin{array}{l}\text { Chlorophyll-a } \\
\text { phyto- } \\
\text { plankton, } \\
\text { chromo- } \\
\text { fluorometer } \\
(\mu g / L)\end{array}$ & $\begin{array}{l}\text { Chlorophyll-b } \\
\text { phyto- } \\
\text { plankton, } \\
\text { chromo- } \\
\text { fluorometer } \\
(\mu \mathrm{g} / \mathrm{L})\end{array}$ & $\begin{array}{l}\text { Sediment, } \\
\text { suspended } \\
\text { (mg/L) }\end{array}$ \\
\hline \multirow[t]{14}{*}{35} & $07-25-88$ & 0540 & 10 & 15 & 3.1 & 96 \\
\hline & $08-09-88$ & 0915 & -- & -- & -- & 30 \\
\hline & $08-30-88$ & 1400 & -- & -- & -- & 105 \\
\hline & $10-18-88$ & 1000 & -- & -- & -- & 69 \\
\hline & $11-08-88$ & 1200 & -- & -- & -- & 32 \\
\hline & $11-14-88$ & 1145 & 7.9 & 62 & 1.1 & 23 \\
\hline & $12-06-88$ & 0900 & -- & -- & -- & 19 \\
\hline & 01-04-89 & 1100 & - & -- & -- & 16 \\
\hline & $01-31-89$ & 1100 & -- & -- & -- & 23 \\
\hline & 03-07-89 & 1435 & 8.9 & 1.2 & .6 & 40 \\
\hline & $03-28-89$ & 1130 & -- & -- & -- & 46 \\
\hline & 04-19-89 & 1130 & -- & -- & -- & 51 \\
\hline & $05-24-89$ & 0940 & -- & -- & -- & 36 \\
\hline & $05-30-89$ & 1245 & 10 & 26 & 4.1 & 22 \\
\hline \multirow[t]{4}{*}{36} & $07-25-88$ & 1230 & 8.5 & 20 & 3.1 & 161 \\
\hline & $11-16-88$ & 1050 & 8.3 & 44 & 1.7 & 41 \\
\hline & 03-09-89 & 0940 & 8.5 & 2.2 & .9 & 48 \\
\hline & $05-31-89$ & 1240 & 7.7 & 72 & 8.1 & 19 \\
\hline \multirow[t]{4}{*}{37} & $07-26-88$ & 0515 & 10 & 18 & 4.7 & 224 \\
\hline & $11-15-88$ & 1100 & 2.1 & 6.2 & .1 & 2 \\
\hline & $03-08-89$ & 0800 & 2.3 & .8 & .5 & 6 \\
\hline & $05-30-89$ & 1200 & 5.6 & .5 & .2 & 1 \\
\hline \multirow[t]{4}{*}{38} & $07-26-88$ & 0810 & 9.7 & 32 & 7.6 & 170 \\
\hline & $11-15-88$ & 0940 & 2.4 & 3.3 & $<.1$ & 3 \\
\hline & 03-08-89 & 0920 & 3.6 & 1.6 & .4 & 88 \\
\hline & $05-30-89$ & 1320 & 3.4 & 1.4 & .3 & 61 \\
\hline \multirow[t]{4}{*}{39} & $07-26-88$ & 0630 & 16 & 70 & 19 & 294 \\
\hline & $11-15-88$ & 0815 & 2.9 & 2.7 & $<.1$ & 25 \\
\hline & 03-08-89 & 1100 & 1.7 & .7 & $<.2$ & 32 \\
\hline & $05-31-89$ & 1030 & 7.0 & 6.2 & .6 & 201 \\
\hline
\end{tabular}


Table 8. Results of ancillary chemical analyses of water samples collected July 1988 through June 1989 in the lower Kansas River Basin-Continued

\begin{tabular}{|c|c|c|c|c|c|c|}
\hline $\begin{array}{c}\text { Site } \\
\text { map-index } \\
\text { number } \\
\text { (fig. 10) }\end{array}$ & Date & Time (24-hour) & $\begin{array}{l}\text { Carbon, } \\
\text { organic, total } \\
\text { (mg/L as C) }\end{array}$ & $\begin{array}{l}\text { Chlorophyll-a } \\
\text { phyto- } \\
\text { plankton, } \\
\text { chromo- } \\
\text { fluorometer } \\
(\mu \mathrm{g} / \mathrm{L})\end{array}$ & $\begin{array}{l}\text { Chlorophyll-b } \\
\text { phyto- } \\
\text { plankton, } \\
\text { chromo- } \\
\text { fluorometer } \\
\text { ( } \mu \mathrm{g} / \mathrm{L})\end{array}$ & $\begin{array}{l}\text { Sediment, } \\
\text { suspended } \\
\text { (mg/L) }\end{array}$ \\
\hline \multirow[t]{4}{*}{40} & $07-26-88$ & 0630 & 16 & 120 & 30 & 149 \\
\hline & $11-14-88$ & 1655 & 2.5 & 3.0 & $<.1$ & 13 \\
\hline & $03-08-89$ & 1345 & 3.7 & .9 & .5 & 77 \\
\hline & $05-31-89$ & 1200 & 8.1 & 6.4 & .7 & 232 \\
\hline 41 & $07-26-88$ & 0600 & 18 & 19 & 4.1 & 700 \\
\hline \multirow[t]{4}{*}{42} & $07-26-88$ & 0545 & 5.2 & 2.9 & .4 & 65 \\
\hline & $11-14-88$ & 1730 & 1.1 & 1.1 & $<.1$ & 6 \\
\hline & $03-08-89$ & 1250 & 1.0 & .5 & .4 & 7 \\
\hline & $05-31-89$ & 1130 & 1.5 & 1.0 & .3 & 10 \\
\hline \multirow[t]{9}{*}{44} & $07-25-88$ & 0845 & 13 & 89 & 24 & 122 \\
\hline & $07-26-88$ & 1320 & -- & - & -- & - \\
\hline & $07-26-88$ & 1710 & - & -- & - & - \\
\hline & $07-26-88$ & 2150 & -- & - & -- & - \\
\hline & $07-27-88$ & 0120 & -- & - & -- & -- \\
\hline & $07-27-88$ & 0545 & - & - & -- & - \\
\hline & $11-14-88$ & 1555 & 2.9 & 1.8 & $<.1$ & 14 \\
\hline & $03-08-89$ & 1225 & 2.9 & .7 & .4 & 28 \\
\hline & $05-31-89$ & 1300 & 5.2 & 9.6 & .9 & 113 \\
\hline \multirow[t]{9}{*}{46} & $07-25-88$ & 0615 & 19 & 5.1 & .9 & 728 \\
\hline & $07-26-88$ & 1355 & -- & -- & -- & - \\
\hline & $07-26-88$ & 1750 & -- & -- & -- & - \\
\hline & $07-26-88$ & 2110 & -- & -- & -- & - \\
\hline & $07-27-88$ & 0100 & -- & -- & -- & - \\
\hline & $07-27-88$ & 0510 & -- & - & -- & - \\
\hline & $11-14-88$ & 1515 & 3.4 & 1.8 & $<.1$ & 94 \\
\hline & $03-08-89$ & 1200 & 4.7 & .6 & $<.2$ & 48 \\
\hline & $05-31-89$ & 1330 & 7.0 & 33 & 5.0 & 176 \\
\hline
\end{tabular}


Table 8. Results of ancillary chemical analyses of water samples collected July 1988 through June 1989 in the lower Kansas River Basin-Continued

\begin{tabular}{|c|c|c|c|c|c|c|}
\hline $\begin{array}{c}\text { Site } \\
\text { map-index } \\
\text { number } \\
\text { (fig. 10) }\end{array}$ & Date & Time (24-hour) & $\begin{array}{c}\text { Carbon, } \\
\text { organic, total } \\
\text { (mg/L as C) }\end{array}$ & $\begin{array}{l}\text { Chlorophyll-a } \\
\text { phyto- } \\
\text { plankton, } \\
\text { chromo- } \\
\text { fluorometer } \\
(\mu g / L)\end{array}$ & $\begin{array}{l}\text { Chlorophyll-b } \\
\text { phyto- } \\
\text { plankton, } \\
\text { chromo- } \\
\text { fluorometer } \\
(\mu g / L)\end{array}$ & $\begin{array}{c}\text { Sediment, } \\
\text { suspended } \\
\text { (mg/L) }\end{array}$ \\
\hline \multirow[t]{19}{*}{48} & $07-25-88$ & 1150 & 12 & 98 & 20 & 197 \\
\hline & $07-27-88$ & 0920 & -- & -- & -- & 134 \\
\hline & 08-09-88 & 1135 & -- & -- & -- & 76 \\
\hline & $08-30-88$ & 1100 & -- & -- & -- & 57 \\
\hline & $10-18-88$ & 1300 & -- & -- & -- & 120 \\
\hline & $11-09-88$ & 1200 & -- & -- & -- & 20 \\
\hline & $11-14-88$ & 1350 & 2.8 & 2.7 & .1 & 16 \\
\hline & $12-06-88$ & 1300 & -- & -- & -- & 21 \\
\hline & $01-10-89$ & 1050 & - & - & -- & 18 \\
\hline & $02-07-89$ & 1115 & -- & - & -- & 46 \\
\hline & 03-07-89 & 1100 & -- & -- & -- & 33 \\
\hline & 03-08-89 & 1040 & 2.9 & .7 & $<.2$ & 47 \\
\hline & $04-05-89$ & 0955 & -- & -- & -- & 79 \\
\hline & $05-01-89$ & 1200 & -- & -- & -- & 51 \\
\hline & $05-31-89$ & 1420 & 5.6 & 17 & 2.1 & 135 \\
\hline & $07-26-88$ & 1215 & 8.7 & 13 & 2.4 & -- \\
\hline & $11-16-88$ & 1400 & 8.0 & 1.6 & .1 & 58 \\
\hline & 03-09-89 & 0830 & 4.1 & 2.7 & .5 & 27 \\
\hline & $05-31-89$ & 1415 & 7.8 & 26 & 6.3 & 59 \\
\hline \multirow[t]{11}{*}{49} & $07-25-88$ & 1400 & 14 & 61 & 15 & 222 \\
\hline & $11-16-88$ & 1130 & 5.0 & 5.7 & .1 & 32 \\
\hline & $11-16-88$ & 1135 & 4.3 & $<.1$ & .2 & 40 \\
\hline & 03-08-89 & 1630 & 3.9 & 1.0 & $<.3$ & 69 \\
\hline & 03-08-89 & 1635 & 4.0 & 1.1 & $<.3$ & 65 \\
\hline & 03-08-89 & 1715 & 4.5 & 1.2 & $<.3$ & 52 \\
\hline & $03-08-89$ & 1720 & 3.9 & 1.1 & .4 & 56 \\
\hline & $05-31-89$ & 1540 & 8.2 & 48 & 6.2 & 122 \\
\hline & $05-31-89$ & 1545 & 7.2 & 57 & 6.9 & 121 \\
\hline & $05-31-89$ & 1550 & 7.6 & 58 & 8.6 & 118 \\
\hline & $05-31-89$ & 1555 & 5.6 & 53 & 7.6 & 122 \\
\hline
\end{tabular}


Table 8. Results of ancillary chemical analyses of water samples collected July 1988 through June 1989 in the lower Kansas River Basin-Continued

\begin{tabular}{|c|c|c|c|c|c|c|}
\hline $\begin{array}{c}\text { Site } \\
\text { map-index } \\
\text { number } \\
\text { (fig. 10) }\end{array}$ & Date & Time (24-hour) & $\begin{array}{l}\text { Carbon, } \\
\text { organic, total } \\
\text { (mg/L as C) }\end{array}$ & $\begin{array}{l}\text { Chlorophyll-a } \\
\text { phyto- } \\
\text { plankton, } \\
\text { chromo- } \\
\text { fluorometer } \\
(\mu \mathrm{g} / \mathrm{L})\end{array}$ & $\begin{array}{l}\text { Chlorophyll-b } \\
\text { phyto- } \\
\text { plankton, } \\
\text { chromo- } \\
\text { fluorometer } \\
(\mu g / L)\end{array}$ & $\begin{array}{l}\text { Sediment, } \\
\text { suspended } \\
\text { (mg/L) }\end{array}$ \\
\hline \multirow[t]{6}{*}{50} & $07-24-88$ & 1320 & 9.4 & 58 & 7.1 & 120 \\
\hline & $11-16-88$ & 1340 & 7.7 & 1.5 & .1 & 93 \\
\hline & $11-16-88$ & 1350 & 7.5 & 1.6 & $<.1$ & 82 \\
\hline & $11-16-88$ & 1355 & -- & -- & -- & -- \\
\hline & 03-09-89 & 1130 & 4.0 & 1.3 & .3 & 60 \\
\hline & $05-31-89$ & 1055 & 8.7 & 46 & 8.7 & 119 \\
\hline \multirow[t]{4}{*}{51} & $07-24-88$ & 1445 & 11 & 40 & 5.0 & 47 \\
\hline & $11-16-88$ & 0940 & 5.2 & 5.3 & .4 & 63 \\
\hline & $03-09-89$ & 1115 & 3.9 & 8.3 & 2.7 & 25 \\
\hline & $05-31-89$ & 1730 & 9.4 & 4.3 & .6 & 12 \\
\hline \multirow[t]{4}{*}{52} & $07-24-88$ & 1045 & 13 & 3.3 & .3 & 28 \\
\hline & $11-15-88$ & 1445 & 5.0 & 3.6 & $<.1$ & 22 \\
\hline & 03-08-89 & 1430 & 4.4 & 2.6 & .2 & 7 \\
\hline & $06-02-89$ & 1030 & 5.1 & 8.9 & 1.1 & 42 \\
\hline \multirow[t]{4}{*}{55} & $07-27-88$ & 0930 & 8.0 & 22 & 3.9 & 48 \\
\hline & $11-15-88$ & 1330 & 8.5 & 11 & .3 & 144 \\
\hline & $03-08-89$ & 1345 & 4.8 & .8 & .7 & 28 \\
\hline & $05-31-89$ & 0900 & 7.5 & 18 & 3.8 & 76 \\
\hline \multirow[t]{4}{*}{56} & $07-27-88$ & 1120 & 5.3 & 11 & 2.1 & 136 \\
\hline & $11-15-88$ & 1115 & 8.0 & 4.5 & .2 & 74 \\
\hline & 03-08-89 & 1100 & 3.6 & .9 & $<.3$ & 46 \\
\hline & $06-01-89$ & 1450 & 9.4 & 52 & 11 & 74 \\
\hline \multirow[t]{3}{*}{57} & $07-29-88$ & 0815 & 32 & 6.6 & 1.4 & 68 \\
\hline & 03-08-89 & 1645 & 2.5 & .8 & $<.3$ & 34 \\
\hline & $06-01-89$ & 1645 & 2.7 & 8.1 & 1.5 & 29 \\
\hline \multirow[t]{4}{*}{58} & $07-27-88$ & 0910 & 6.9 & 34 & 3.7 & 59 \\
\hline & $11-15-88$ & 1445 & 6.4 & 16 & .4 & 76 \\
\hline & $03-10-89$ & 1110 & 8.0 & 16 & 21 & -- \\
\hline & $05-30-89$ & 1845 & 15 & 32 & 4.6 & 436 \\
\hline
\end{tabular}


Table 8. Results of ancillary chemical analyses of water samples collected July 1988 through June 1989 in the lower Kansas River Basin-Continued

\begin{tabular}{|c|c|c|c|c|c|c|}
\hline $\begin{array}{c}\text { Site } \\
\text { map-index } \\
\text { number } \\
\text { (fig. 10) }\end{array}$ & Date & Time (24-hour) & $\begin{array}{l}\text { Carbon, } \\
\text { organic, total } \\
\text { (mg/L as C) }\end{array}$ & $\begin{array}{c}\text { Chlorophyll-a } \\
\text { phyto- } \\
\text { plankton, } \\
\text { chromo- } \\
\text { fluorometer } \\
(\mu g / L)\end{array}$ & $\begin{array}{c}\text { Chlorophyll-b } \\
\text { phyto- } \\
\text { plankton, } \\
\text { chromo- } \\
\text { fluorometer } \\
(\mu g / L)\end{array}$ & $\begin{array}{l}\text { Sediment, } \\
\text { suspended } \\
\text { (mg/L) }\end{array}$ \\
\hline \multirow[t]{4}{*}{59} & $07-27-88$ & 1010 & 4.0 & 11 & 2.1 & 109 \\
\hline & $11-15-88$ & 1100 & 3.7 & 2.0 & .1 & 21 \\
\hline & 03-08-89 & 1310 & 2.6 & 1.3 & .7 & 69 \\
\hline & $06-01-89$ & 1145 & 5.1 & 9.8 & 1.1 & 65 \\
\hline \multirow[t]{4}{*}{60} & $07-29-88$ & 0535 & 32 & 23 & 3.6 & 179 \\
\hline & $11-15-88$ & 1240 & 8.5 & 9.3 & $<.1$ & 35 \\
\hline & 03-09-89 & 1230 & 3.0 & 3.2 & 1.0 & 20 \\
\hline & $06-01-89$ & 1310 & 7.5 & 23 & 3.8 & 79 \\
\hline \multirow[t]{5}{*}{61} & $07-28-88$ & 1745 & -- & -- & -- & -- \\
\hline & $07-29-88$ & 0415 & 8.6 & 44 & 4.4 & 109 \\
\hline & $11-15-88$ & 1030 & 6.5 & 12 & .3 & 139 \\
\hline & 03-08-89 & 1030 & 5.2 & 3.7 & 2.3 & 47 \\
\hline & $05-31-89$ & 1710 & 12 & 61 & 7.7 & 122 \\
\hline \multirow[t]{6}{*}{6.2} & $07-28-88$ & 1815 & -- & -- & -- & - \\
\hline & $07-29-88$ & 0530 & 10 & 27 & 4.1 & 198 \\
\hline & $07-29-88$ & 0535 & 8.9 & 38 & 5.7 & 199 \\
\hline & $11-15-88$ & 0755 & 5.6 & 6.8 & .3 & 41 \\
\hline & 03-07-89 & 1510 & 6.9 & 4.9 & .7 & 94 \\
\hline & 06-01-89 & 1815 & 11 & 65 & 10 & 35 \\
\hline \multirow[t]{4}{*}{63} & $07-29-88$ & 0910 & 4.9 & 23 & 9.4 & 48 \\
\hline & $11-14-88$ & 1000 & 3.7 & .5 & $<.1$ & 68 \\
\hline & 03-07-89 & 1010 & 3.2 & 2.9 & .1 & 28 \\
\hline & $06-01-89$ & 1300 & 6.9 & 18 & 1.8 & 52 \\
\hline \multirow[t]{5}{*}{64} & $07-28-88$ & 1230 & 5.7 & 13 & 5.8 & 66 \\
\hline & $07-28-88$ & 1235 & 5.7 & 12 & 4.7 & 98 \\
\hline & $07-28-88$ & 1830 & -- & -- & -- & -- \\
\hline & $07-29-88$ & 0005 & -- & -- & -- & -- \\
\hline & $07-29-88$ & 0435 & -- & -- & -- & -- \\
\hline
\end{tabular}


Table 8. Results of ancillary chemical analyses of water samples collected July 1988 through June 1989 in the lower Kansas River Basin-Continued

\begin{tabular}{|c|c|c|c|c|c|c|}
\hline $\begin{array}{c}\text { Site } \\
\text { map-index } \\
\text { number } \\
\text { (fig. 10) }\end{array}$ & Date & Time (24-hour) & $\begin{array}{c}\text { Carbon, } \\
\text { organic, total } \\
\text { (mg/L as C) }\end{array}$ & $\begin{array}{c}\text { Chlorophyll-a } \\
\text { phyto- } \\
\text { plankton, } \\
\text { chromo- } \\
\text { fluorometer } \\
(\mu \mathrm{g} / \mathrm{L})\end{array}$ & $\begin{array}{l}\text { Chlorophyll-b } \\
\text { phyto- } \\
\text { plankton, } \\
\text { chromo- } \\
\text { fluorometer } \\
(\mu g / L)\end{array}$ & $\begin{array}{l}\text { Sediment, } \\
\text { suspended } \\
\text { (mg/L) }\end{array}$ \\
\hline \multirow[t]{7}{*}{64} & $11-14-88$ & 1530 & 4.2 & 9.3 & 0.3 & 19 \\
\hline & 03-07-89 & 1230 & 5.9 & 4.2 & .6 & 37 \\
\hline & 03-07-89 & 1235 & 4.0 & 3.8 & .6 & 46 \\
\hline & $03-07-89$ & 1330 & 3.1 & 2.7 & .5 & 37 \\
\hline & 03-07-89 & 1335 & 3.0 & 2.4 & .3 & 62 \\
\hline & $05-31-89$ & 1430 & 7.8 & 32 & 6.1 & 32 \\
\hline & $05-31-89$ & 1435 & 7.6 & 31 & 5.3 & 30 \\
\hline \multirow[t]{4}{*}{71} & $07-27-88$ & 0610 & 6.0 & 15 & 2.1 & 130 \\
\hline & $11-17-88$ & 1230 & 6.3 & 1.1 & $<.1$ & 24 \\
\hline & 03-09-89 & 1100 & 6.7 & 2.2 & .5 & 3 \\
\hline & $06-01-89$ & 1445 & 9.1 & 42 & 6.8 & 76 \\
\hline \multirow[t]{13}{*}{72} & $07-27-88$ & 0745 & 6.0 & 17 & 3.5 & 48 \\
\hline & $08-31-88$ & 1025 & -- & -- & -- & 42 \\
\hline & $10-05-88$ & 1000 & -- & -- & -- & 16 \\
\hline & $11-09-88$ & 1020 & -- & -- & -- & -- \\
\hline & $11-17-88$ & 1020 & 8.4 & 5.5 & .2 & 38 \\
\hline & $12-21-88$ & 1010 & -- & -- & -- & 27 \\
\hline & $01-25-89$ & 1220 & -- & -- & -- & -- \\
\hline & $02-15-89$ & 1035 & -- & -- & -- & -- \\
\hline & $03-09-89$ & 1230 & 5.9 & 3.5 & .4 & 9 \\
\hline & $03-15-89$ & 1135 & -- & -- & -- & -- \\
\hline & $04-19-89$ & 0925 & -- & -- & -- & -- \\
\hline & $05-17-89$ & 1320 & -- & -- & -- & -- \\
\hline & $06-02-89$ & 0945 & 8.5 & 22 & 3.5 & 24 \\
\hline \multirow[t]{5}{*}{73} & $07-27-88$ & 0810 & 5.9 & 4.4 & 1.6 & -- \\
\hline & $11-17-88$ & 1300 & 11 & 11 & .5 & 21 \\
\hline & $11-17-88$ & 1305 & 11 & 7.3 & .3 & 26 \\
\hline & $11-17-88$ & 1345 & 15 & 7.6 & .2 & 29 \\
\hline & $11-17-88$ & 1350 & 14 & 8.8 & .3 & 22 \\
\hline
\end{tabular}


Table 8. Results of ancillary chemical analyses of water samples collected July 1988 through June 1989 in the lower Kansas River Basin-Continued

\begin{tabular}{|c|c|c|c|c|c|c|}
\hline $\begin{array}{c}\text { Site } \\
\text { map-index } \\
\text { number } \\
\text { (fig. 10) }\end{array}$ & Date & Time (24-hour) & $\begin{array}{c}\text { Carbon, } \\
\text { organic, total } \\
\text { (mg/L as C) }\end{array}$ & $\begin{array}{l}\text { Chlorophyll-a } \\
\text { phyto- } \\
\text { plankton, } \\
\text { chromo- } \\
\text { fluorometer } \\
(\mu g / L)\end{array}$ & $\begin{array}{l}\text { Chlorophyll-b } \\
\text { phyto- } \\
\text { plankton, } \\
\text { chromo- } \\
\text { fluorometer } \\
(\mu g / L)\end{array}$ & $\begin{array}{c}\text { Sediment, } \\
\text { suspended } \\
\text { (mg/L) }\end{array}$ \\
\hline \multirow[t]{6}{*}{73} & 03-09-89 & 1400 & 6.1 & 6.8 & 1.2 & 10 \\
\hline & $03-09-89$ & 1405 & 7.1 & 6.9 & 1.4 & 12 \\
\hline & $03-09-89$ & 1440 & 6.7 & 6.7 & 1.0 & 14 \\
\hline & 03-09-89 & 1445 & 6.7 & 6.8 & 1.3 & 13 \\
\hline & $06-02-89$ & 1100 & 7.1 & 2.3 & .4 & 13 \\
\hline & $06-02-89$ & 1105 & -- & 2.0 & .4 & 13 \\
\hline \multirow[t]{14}{*}{74} & $07-29-88$ & 1020 & 6.2 & 1.7 & .4 & 44 \\
\hline & $08-10-88$ & 1300 & -- & -- & -- & 52 \\
\hline & $08-31-88$ & 1320 & -- & -- & -- & 10 \\
\hline & $10-05-88$ & 1300 & -- & -- & -- & 11 \\
\hline & $11-09-88$ & 1310 & -- & -- & -- & -- \\
\hline & $11-15-88$ & 1430 & 4.8 & 2.1 & $<.1$ & 12 \\
\hline & $12-21-88$ & 1315 & -- & -- & -- & 7 \\
\hline & $01-25-89$ & 0845 & -- & -- & - & - \\
\hline & $02-15-89$ & 1335 & -- & -- & -- & -- \\
\hline & 03-07-89 & 0800 & 5.9 & 5.5 & 1.3 & 5 \\
\hline & $03-15-89$ & 0845 & -- & -- & -- & -- \\
\hline & $04-19-89$ & 1235 & -- & -- & -- & -- \\
\hline & $05-17-89$ & 1045 & -- & -- & -- & -- \\
\hline & $05-31-89$ & 1630 & 5.7 & .9 & .2 & 55 \\
\hline \multirow[t]{5}{*}{76} & $07-27-88$ & 1805 & -- & -- & - & -- \\
\hline & $07-28-88$ & 0025 & -- & -- & -- & -- \\
\hline & $07-28-88$ & 0615 & 10 & 75 & 10 & 90 \\
\hline & $07-28-88$ & 1215 & -- & -- & -- & -- \\
\hline & $07-28-88$ & 1220 & -- & -- & -- & -- \\
\hline
\end{tabular}


Table 8. Results of ancillary chemical analyses of water samples collected July 1988 through June 1989 in the lower Kansas River Basin-Continued

\begin{tabular}{|c|c|c|c|c|c|c|}
\hline $\begin{array}{c}\text { Site } \\
\text { map-index } \\
\text { number } \\
\text { (fig. 10) }\end{array}$ & Date & Time (24-hour) & $\begin{array}{c}\text { Carbon, } \\
\text { organic, total } \\
\text { (mg/L as C) }\end{array}$ & $\begin{array}{l}\text { Chlorophyll-a } \\
\text { phyto- } \\
\text { plankton, } \\
\text { chromo- } \\
\text { fluorometer } \\
(\mu \mathrm{g} / \mathrm{L})\end{array}$ & $\begin{array}{c}\text { Chlorophyll-b } \\
\text { phyto- } \\
\text { plankton, } \\
\text { chromo- } \\
\text { fluorometer } \\
(\mu \mathrm{g} / \mathrm{L})\end{array}$ & $\begin{array}{l}\text { Sediment, } \\
\text { suspended } \\
\text { (mg/L) }\end{array}$ \\
\hline \multirow[t]{28}{*}{76} & $11-14-88$ & 0600 & -- & -- & -- & - \\
\hline & $11-14-88$ & 0610 & -- & -- & - & - \\
\hline & $11-14-88$ & 1000 & -- & -- & -- & 26 \\
\hline & $11-14-88$ & 1005 & -- & -- & -- & 31 \\
\hline & $11-14-88$ & 1400 & 5.6 & -- & -- & 26 \\
\hline & $11-14-88$ & 1410 & -- & -- & -- & -- \\
\hline & $11-14-88$ & 1800 & -- & -- & -- & -- \\
\hline & $11-14-88$ & 1805 & -- & -- & -- & -- \\
\hline & $11-14-88$ & 2200 & -- & -- & -- & -- \\
\hline & $11-14-88$ & 2205 & -- & -- & - & - \\
\hline & $11-15-88$ & 0200 & -- & -- & -- & -- \\
\hline & $11-15-88$ & 0205 & -- & -- & -- & -- \\
\hline & $11-15-88$ & 0605 & -- & -- & -- & -- \\
\hline & 03-07-89 & 0615 & -- & -- & - & -- \\
\hline & 03-07-89 & 1010 & -- & -- & -- & -- \\
\hline & 03-07-89 & 1015 & -- & -- & -- & -- \\
\hline & 03-07-89 & 1400 & 5.9 & 5.7 & 0.5 & 59 \\
\hline & 03-07-89 & 1405 & 5.8 & 5.8 & .5 & 42 \\
\hline & 03-07-89 & 1410 & 5.4 & 4.8 & .5 & 52 \\
\hline & 03-07-89 & 1800 & -- & -- & -- & -- \\
\hline & 03-07-89 & 1805 & -- & -- & -- & -- \\
\hline & 03-07-89 & 2200 & -- & -- & -- & -- \\
\hline & 03-07-89 & 2205 & -- & - & -- & -- \\
\hline & 03-08-89 & 0200 & -- & -- & -- & -- \\
\hline & $03-08-89$ & 0205 & -- & -- & -- & -- \\
\hline & 03-08-89 & 0600 & -- & -- & -- & -- \\
\hline & 03-08-89 & 0605 & -- & -- & -- & -- \\
\hline & $05-31-89$ & 1845 & 14 & 46 & 4.6 & 93 \\
\hline
\end{tabular}


Table 8. Results of ancillary chemical analyses of water samples collected July 1988 through June 1989 in the lower Kansas River Basin-Continued

\begin{tabular}{|c|c|c|c|c|c|c|}
\hline $\begin{array}{c}\text { Site } \\
\text { map-index } \\
\text { number } \\
\text { (fig. 10) }\end{array}$ & Date & Time (24-hour) & $\begin{array}{c}\text { Carbon, } \\
\text { organic, total } \\
\text { (mg/L as C) }\end{array}$ & $\begin{array}{c}\text { Chlorophyll-a } \\
\text { phyto- } \\
\text { plankton, } \\
\text { chromo- } \\
\text { fluorometer } \\
(\mu \mathrm{g} / \mathrm{L})\end{array}$ & $\begin{array}{l}\text { Chlorophyll-b } \\
\text { phyto- } \\
\text { plankton, } \\
\text { chromo- } \\
\text { fluorometer } \\
(\mu \mathrm{g} / \mathrm{L})\end{array}$ & $\begin{array}{l}\text { Sediment, } \\
\text { suspended } \\
\text { (mg/L) }\end{array}$ \\
\hline \multirow[t]{5}{*}{77} & $07-27-88$ & 1825 & -- & -- & -- & -- \\
\hline & $07-28-88$ & 0605 & 9.3 & 30 & 5.1 & 103 \\
\hline & $11-14-88$ & 1030 & 5.6 & 22 & 1.0 & 28 \\
\hline & 03-07-89 & 1345 & 5.8 & 2.7 & .8 & 188 \\
\hline & 06-03-89 & 0935 & 11 & 67 & 6.7 & 125 \\
\hline \multirow[t]{4}{*}{78} & $07-28-88$ & 0515 & 8.0 & 34 & 5.5 & 42 \\
\hline & $11-14-88$ & 1440 & 5.9 & 7.8 & .4 & 20 \\
\hline & 03-07-89 & 1030 & 6.4 & 1.7 & .7 & 31 \\
\hline & $06-01-89$ & 1800 & 10 & 52 & 6.1 & -- \\
\hline \multirow[t]{3}{*}{79} & $07-29-88$ & 0645 & 7.2 & 16 & 2.7 & 28 \\
\hline & 03-07-89 & 1600 & 8.0 & 3.2 & .2 & 22 \\
\hline & $05-31-89$ & 0930 & 9.0 & 3.8 & .5 & 22 \\
\hline \multirow[t]{14}{*}{83} & $07-29-88$ & 0530 & 6.3 & 12 & 0.8 & 48 \\
\hline & $08-10-88$ & 1500 & -- & -- & -- & 44 \\
\hline & $08-31-88$ & 1500 & -- & -- & -- & 90 \\
\hline & $10-05-88$ & 1500 & -- & -- & -- & 24 \\
\hline & $11-09-88$ & 1555 & -- & -- & -- & - \\
\hline & $11-16-88$ & 1520 & -- & -- & -- & -- \\
\hline & $12-21-88$ & 1515 & -- & -- & -- & 6 \\
\hline & $01-25-89$ & 1605 & -- & -- & -- & -- \\
\hline & $02-15-89$ & 1535 & -- & -- & -- & -- \\
\hline & 03-07-89 & 1300 & 5.5 & 1.3 & .1 & 9 \\
\hline & 03-15-89 & 1435 & -- & -- & -- & -- \\
\hline & $04-19-89$ & 1450 & -- & -- & -- & -- \\
\hline & $05-17-89$ & 0755 & -- & -- & -- & -- \\
\hline & $05-31-89$ & 1045 & 6.1 & 7.5 & .5 & 92 \\
\hline \multirow[t]{5}{*}{86} & $07-28-88$ & 0615 & 9.8 & 12 & 1.3 & 56 \\
\hline & $07-28-88$ & 0620 & 9.8 & 11 & 1.2 & 56 \\
\hline & $07-28-88$ & 1200 & 14 & 93 & 14 & -- \\
\hline & $03-08-89$ & 1200 & 5.2 & 3.6 & .7 & 13 \\
\hline & $06-02-89$ & 1250 & 9.1 & 11 & 2.6 & 10 \\
\hline
\end{tabular}


Table 8. Results of ancillary chemical analyses of water samples collected July 1988 through June 1989 in the lower Kansas River Basin-Continued

\begin{tabular}{|c|c|c|c|c|c|c|}
\hline $\begin{array}{c}\text { Site } \\
\text { map-Index } \\
\text { number } \\
\text { (fig. 10) }\end{array}$ & Date & Time (24-hour) & $\begin{array}{l}\text { Carbon, } \\
\text { organic, total } \\
\text { (mg/L as C) }\end{array}$ & $\begin{array}{l}\text { Chlorophyll-a } \\
\text { phyto- } \\
\text { plankton, } \\
\text { chromo- } \\
\text { fluorometer } \\
(\mu \mathrm{g} / \mathrm{L})\end{array}$ & $\begin{array}{l}\text { Chlorophyll-b } \\
\text { phyto- } \\
\text { plankton, } \\
\text { chromo- } \\
\text { fluorometer } \\
(\mu g / L)\end{array}$ & $\begin{array}{l}\text { Sediment, } \\
\text { suspended } \\
\text { (mg/L) }\end{array}$ \\
\hline \multirow[t]{6}{*}{87} & $07-28-88$ & 0445 & 10 & 16 & 2.2 & 75 \\
\hline & $07-28-88$ & 1500 & -- & -- & -- & -- \\
\hline & $11-14-88$ & 1345 & 9.8 & 1.5 & .2 & 32 \\
\hline & $11-14-88$ & 1350 & 10 & 8.8 & .8 & 34 \\
\hline & 03-08-89 & 1415 & 5.2 & 3.5 & .4 & 12 \\
\hline & $06-02-89$ & 1530 & 7.0 & 23 & 4.6 & 286 \\
\hline \multirow[t]{13}{*}{88} & $07-27-88$ & 1815 & -- & -- & -- & -- \\
\hline & $07-27-88$ & 2400 & -- & -- & -- & -- \\
\hline & $07-28-88$ & 0610 & 8.2 & 40 & 7.7 & 47 \\
\hline & $07-28-88$ & 1220 & -- & -- & -- & -- \\
\hline & $07-28-88$ & 1225 & -- & -- & -- & -- \\
\hline & $11-16-88$ & 1510 & 5.9 & 9.5 & 0.6 & 34 \\
\hline & $11-16-88$ & 1515 & 5.8 & 9.2 & .5 & 33 \\
\hline & $03-07-89$ & 1550 & 6.8 & 3.0 & 1.0 & 14 \\
\hline & 03-07-89 & 1555 & 6.5 & 3.5 & .8 & 16 \\
\hline & 03-07-89 & 1630 & 6.3 & 2.8 & .8 & 20 \\
\hline & 03-07-89 & 1635 & 6.4 & 2.9 & 1.1 & 14 \\
\hline & $05-30-89$ & 1905 & 8.5 & 51 & 5.7 & 15 \\
\hline & $05-30-89$ & 1910 & 9.2 & 57 & 8.0 & 15 \\
\hline \multirow[t]{8}{*}{90} & $07-29-88$ & 0510 & 23 & 3.3 & .6 & 10 \\
\hline & $07-29-88$ & 0515 & 19 & 2.5 & .8 & 11 \\
\hline & $11-14-88$ & 1210 & 25 & .3 & .1 & 9 \\
\hline & $11-14-88$ & 1215 & 27 & .3 & $<.1$ & 12 \\
\hline & $11-14-88$ & 1315 & - & .2 & $<.1$ & -- \\
\hline & $11-14-88$ & 1320 & -- & .5 & $<.1$ & -- \\
\hline & 03-07-89 & 0925 & 32 & 2.0 & 1.3 & 17 \\
\hline & $06-02-89$ & 1440 & 18 & .7 & .1 & -- \\
\hline \multirow[t]{4}{*}{91} & $07-29-88$ & 0615 & 9.9 & 40 & 5.5 & 219 \\
\hline & $11-14-88$ & 1000 & 7.0 & 16 & .6 & 34 \\
\hline & 03-07-89 & 1320 & 10 & 9.7 & 3.1 & 24 \\
\hline & $06-02-89$ & 1145 & 8.3 & 23 & 3.2 & -- \\
\hline
\end{tabular}

